\title{
FKT is Not Universal - A Planar Holant Dichotomy for Symmetric Constraints
}

\author{
Jin-Yi Cai ${ }^{1} \cdot$ Zhiguo Fu $^{2} \cdot$ Heng Guo $^{3}$ (D) $\cdot$ Tyson Williams $^{4}$
}

Accepted: 11 January 2021 / Published online: 9 August 2021

(C) The Author(s) 2021

\begin{abstract}
We prove a complexity classification for Holant problems defined by an arbitrary set of complex-valued symmetric constraint functions on Boolean variables. This is to specifically answer the question: Is the Fisher-Kasteleyn-Temperley (FKT) algorithm under a holographic transformation (Valiant, SIAM J. Comput. 37(5), 1565-1594 2008) a universal strategy to obtain polynomial-time algorithms for problems over planar graphs that are intractable on general graphs? There are problems that are \#P-hard on general graphs but polynomial-time solvable on planar graphs. For spin systems (Kowalczyk 2010) and counting constraint satisfaction problems (\#CSP) (Guo and Williams, J. Comput. Syst. Sci. 107, 1-27 2020), a recurring theme has emerged that a holographic reduction to FKT precisely captures these problems. Surprisingly, for Holant, we discover new planar tractable problems that are not expressible by a holographic reduction to FKT. In particular, a straightforward formulation of a dichotomy for planar Holant problems along the above recurring theme is false. A dichotomy theorem for \#CSP ${ }^{d}$, which denotes \#CSP where every variable appears a multiple of $d$ times, has been an important tool in previous work. However the proof for the \#CSP $^{d}$ dichotomy violates planarity, and it does not generalize to the planar case easily. In fact, due to our newly discovered tractable problems, the putative form of a planar \#CSP ${ }^{d}$ dichotomy is false when $d \geq 5$. Nevertheless, we prove a dichotomy for planar \#CSP${ }^{2}$. In this case, the putative form of the dichotomy is true. (This is presented in Part II of the paper.) We manage to prove
\end{abstract}

A preliminary version appeared in FOCS 2015 [9].

Supported by NSF CCF-1714275.

Supported by National Natural Science Foundation of China (Grant No. 61872076).

This project has received funding from the European Research Council (ERC) under the European Union's Horizon 2020 research innovation programme (grant agreement No. 947778).

Heng Guo

hguo@inf.ed.ac.uk

Extended author information available on the last page of the article. 
the planar Holant dichotomy relying only on this planar \#CSP${ }^{2}$ dichotomy, without resorting to a more general planar $\mathrm{ACSP}^{d}$ dichotomy for $d \geq 3$. A special case of the new polynomial-time computable problems is counting perfect matchings (\#PM) over $k$-uniform hypergraphs when the incidence graph is planar and $k \geq 5$. The same problem is \#P-hard when $k=3$ or $k=4$, which is also a consequence of our dichotomy. When $k=2$, it becomes \#PM over planar graphs and is tractable again. More generally, over hypergraphs with specified hyperedge sizes and the same planarity assumption, \#PM is polynomial-time computable if the greatest common divisor ( $\mathrm{gcd}$ ) of all hyperedge sizes is at least 5. It is worth noting that it is the gcd, and not a bound on hyperedge sizes, that is the criterion for tractability.

Keywords Computational complexity $\cdot$ Counting $\cdot$ Holographic algorithms

\section{Part I Planar Holant Dichotomy}

\section{Introduction}

The Fisher-Kasteleyn-Temperley (FKT) algorithm [29, 30, 41] is a classical gem that counts perfect matchings over planar graphs in polynomial time. This was an important milestone in a decades-long research program by physicists in statistical mechanics to determine what is known as Exactly Solved Models [3, 28-30, 33-35, 39, 41, 49-51].

For four decades, the FKT algorithm stood as the polynomial-time algorithm for any counting problem over planar graphs that is \#P-hard over general graphs. Then Valiant introduced matchgates [42, 43] and holographic reductions to the FKT algorithm [44, 45]. These reductions differ from classical ones by introducing quantum-like superpositions. This novel technique extended the reach of the FKT algorithm and produced polynomial-time algorithms for a number of problems for which only exponential-time algorithms were previously known.

Since the new polynomial-time algorithms appear so exotic and unexpected, and since they solve problems that appear so close to being \#P-hard, they challenge our faith in the well-accepted conjecture that $\mathrm{P} \neq \mathrm{NP}$. Quoting Valiant [44]: "The objects enumerated are sets of polynomial systems such that the solvability of any one member would give a polynomial time algorithm for a specific problem. ... the situation with the $\mathrm{P}=\mathrm{NP}$ question is not dissimilar to that of other unresolved enumerative conjectures in mathematics. The possibility that accidental or freak objects in the enumeration exist cannot be discounted if the objects in the enumeration have not been studied systematically." Indeed, if any "freak" object exists in this framework, it would collapse \#P to P. Therefore, over the past 10 to 15 years, this technique has been intensely studied in order to gain a systematic understanding of the limit of the trio of holographic reductions, matchgates, and the FKT algorithm [6, 7, 15, 16, 32, 37, 38, 42, 46]. Without settling the $\mathrm{P}$ versus \#P question, the best hope is to achieve a complexity classification. This program finds its sharpest expression in a 
complexity dichotomy theorem, which classifies every problem expressible in a framework as either solvable in $\mathrm{P}$ or \#P-hard, with nothing in between.

Out of this work, a strong theme has emerged. For a wide variety of problems, such as those expressible as a \#CSP, holographic reductions to the FKT algorithm is a universal technique for turning problems that are \#P-hard in general to P-time solvable over planar graphs. In fact, a preponderance of evidence suggests the following putative classification of all counting problems defined by local constraints into exactly three categories: (1) those that are P-time solvable over general graphs; (2) those that are P-time solvable over planar graphs but \#P-hard over general graphs; and (3) those that remain \#P-hard over planar graphs. Moreover, category (2) consists precisely of those problems that are holographically reducible to the FKT algorithm. This theme is so strong that it has become an intuitive and trusty guide for us when we investigate unknown problems and plan proof strategies. In fact, many of the results in the present paper are proved in this way. However, one is still left wondering whether the FKT algorithm is universal, or more precisely, is the combined algorithmic power of holographic reductions, matchgates, and the FKT algorithm sufficient to capture all tractable problems over planar graphs that are intractable in general?

We list some of the supporting evidence for this putative classification. These date back to the classification of the complexity of the Tutte polynomial [47, 48]. It has also been an unfailing theme in the classification of spin systems and \#CSP [14, 19, 26, 31]. However, these frameworks do not capture all locally specified counting problems. Some natural problems, such as counting perfect matchings (\#PM), are not expressible as a point on the Tutte polynomial or a \#CSP, and \#PM is provably not expressible within the special case of vertex assignment models [23, 24, 40]. However, this is the problem for which FKT was designed, and is the basis of Valiant's matchgates and holographic reductions.

A refined framework, called Holant problems [17], was proposed to address this issue. It is an edge assignment model. It naturally encodes and expresses \#PM as well as Valiant's matchgates and holographic reductions. Thus, Holant is the proper framework in which to study the power of holographic algorithms. It is also more general than \#CSP in that any \#CSP problem is a special case of a Holant problem, and a complete complexity classification for Holant problems implies one for \#CSP.

In this paper, we classify for the first time the complexity of Holant problems over planar graphs with an arbitrary set of symmetric complex-valued constraint functions. Our result generalizes both the dichotomy for Holant [11, 27] and the dichotomy for planar \#CSP $[19,26]$. Surprisingly, we discover new planar tractable problems that are not expressible by a holographic reduction to matchgates and FKT. To the best of our knowledge, this is the first primitive extension since FKT to a problem solvable in $\mathrm{P}$ over planar instances but \#P-hard in general. We consider this extension primitive in the sense that it provably cannot be obtained by applying any holographic reduction to matchgates and FKT. Furthermore, our dichotomy theorem says that this completes the picture: there are no more undiscovered extensions for problems expressible in this framework, unless \#P collapses to P. In particular, the putative form of the planar Holant dichotomy is false.

Before stating our main theorem, we give a brief description of the Holant framework [17]. Fix a set of local constraint functions $\mathcal{F}$. A signature grid $\Omega=(G, \pi)$ is 
a tuple, where $G=(V, E)$ is a graph, $\pi$ labels each $v \in V$ with a function $f_{v} \in \mathcal{F}$ with input variables from the incident edges $E(v)$ at $v$. Each $f_{v}$ maps $\{0,1\}^{\operatorname{deg}(v)}$ to $\mathbb{C}$. We consider all 0-1 edge assignments. An assignment $\sigma$ for every $e \in E$ gives an evaluation $\prod_{v \in V} f_{v}\left(\left.\sigma\right|_{E(v)}\right)$, where $\left.\sigma\right|_{E(v)}$ denotes the restriction of $\sigma$ to $E(v)$. The counting problem on the instance $\Omega$ is to compute

$$
\operatorname{Holant}(\Omega ; \mathcal{F})=\sum_{\sigma: E \rightarrow\{0,1\}} \prod_{v \in V} f_{v}\left(\left.\sigma\right|_{E(v)}\right) .
$$

For example, \#PM, the problem of counting perfect matchings in $G$, corresponds to assigning the ExACTONE function at every vertex of $G$. The Holant problem parameterized by the set $\mathcal{F}$ is denoted by $\operatorname{Holant}(\mathcal{F})$.

At a high level, we can state our main theorem as follows.

Theorem 1.1 Let $\mathcal{F}$ be a set of complex-valued, symmetric functions on Boolean variables. Then there is an effective classification for all possible $\mathcal{F}$, according to which, Holant $(\mathcal{F})$ is either (1) P-time computable over general graphs, or (2) P-time computable over planar graphs but \#P-hard over general graphs, or (3) \#P-hard over planar graphs.

The complete statement is given in Theorem 8.1. The classification is explicit. The tractability criterion is decidable in polynomial time due to $[12,16]$. Tractable problems over general graphs have been previously studied in [11]. The planar tractable class includes both those solvable by holographic reductions to FKT and those newly discovered. Explicit criteria for these are also proved in this paper.

Let us meet some new tractable problems. We consider some orientation problems, which are Holant problems after a complex-valued holographic transformation. ${ }^{1}$ Given a planar graph, we allow two kinds of vertices. The first kind can be either a sink or a source while the second kind must have exactly one incoming edge. The goal is to compute the number of orientations satisfying these constraints. This problem can be expressed in the Holant framework under a $Z$-transformation, where $Z=$ $\left[\begin{array}{ll}1 & 1 \\ i & -i\end{array}\right]$. It can be shown that this is equivalent to the Holant problem on the edgevertex incidence graph where we assign the DISEQUALITY function to every edge, we assign either the EQUALITY function or the EXACTONE function to each vertex. Suppose vertices assigned EQUALITY functions all have degree $k$. If $k=2$, then this problem can be solved by FKT. We show that this problem is \#P-hard if $k=3$ or $k=4$, but is tractable again if $k \geq 5$. The algorithm involves a recursive procedure that simplifies the instance until it can be solved by known algorithms, including FKT. The algorithm crucially uses global topological properties of planar graphs, in

\footnotetext{
${ }^{1}$ This transformation is $Z=\left[\begin{array}{ll}1 & 1 \\ i & -i\end{array}\right]$. It is common that one problem can be transformed to another over $\mathbb{C}$ while one or both problems are specified by real-valued constraint functions, and provably no transformation exists over $\mathbb{R}$. Thus it is both natural and proper to study the classification question over complex-valued constraint functions. For example, the integer-valued orientation problem studied here is complex weighted if expressed directly as Holant.
} 
particular Euler's characteristic formula. If the graph is not planar, then this algorithm does not work, and indeed the problem is \#P-hard over general graphs.

More generally, we allow vertices of arbitrary degrees to be assigned EQUALITY. If all the degrees are at most 2 , then the problem is tractable by the FKT algorithm. Otherwise, the complexity depends on the greatest common divisor (gcd) of the degrees. The problem is tractable if gcd $\geq 5$ and \#P-hard if gcd $\leq 4$. It is worth noting that the criterion for tractability is not a degree lower bound. Moreover, the planarity assumption and the degree rigidity pose a formidable challenge in the hardness proofs for gcd $\leq 4$.

If the graph is bipartite with EQUALITY functions assigned on one side and EXACTONE functions on the other, then this is the problem of \#PM over hypergraphs with planar incidence graphs. Our results imply that the complexity of this problem depends on the gcd of the hyperedge sizes. The problem is computable in polynomial time when gcd $\geq 5$ and is \#P-hard when gcd $\leq 4$ (assuming there are hyperedges of size at least 3). For a formal statement, see Theorem 7.17.

Most of the reductions in previous Holant dichotomy theorems [11, 27] do not hold for planar graphs, so we are forced to develop new techniques and formulate new proof strategies. In particular, an important ingredient in previous proofs is the \#CSP ${ }^{d}$ dichotomy by Huang and Lu [27]. Here \#CSP ${ }^{d}$ denotes \#CSP where every variable appears a multiple of $d$ times. The very first step in the \#CSP ${ }^{d}$ dichotomy proof uses a pinning technique. In this proof multiple copies of a graph are created and vertices are connected across different copies. But this construction fundamentally violates planarity. Moreover, as a consequence of the new dichotomy, this violation of planarity is unavoidable. Owing to our newly discovered tractable problems, the putative form of a planar \#CSP ${ }^{d}$ dichotomy is false when $d \geq 5$. Nevertheless, we prove a dichotomy for planar \#CSP ${ }^{2}$ for which the putative form is true (which is lucky for us but not obvious in hindsight). Obtaining a dichotomy for planar \#CSP ${ }^{2}$ is essential because it captures a significant fraction of planar Holant problems either directly or through reductions. We manage to prove the planar Holant dichotomy without appealing to planar \#CSP ${ }^{d}$ for $d \geq 3$.

The proof of the planar \#CSP ${ }^{2}$ dichotomy comprises the entire Part II of this paper. A brief outline of this proof of the planar \#CSP${ }^{2}$ dichotomy is given in Section 5 of Part I. Among the concepts and techniques introduced are some special tractable families of constraint functions specific to the \#CSP ${ }^{2}$ framework. We also introduce a derivative operator $\partial$ and its inverse operator integral $\int$ to streamline the proof argument. It also uses some elementary properties of cyclotomic fields.

We began this project expecting to prove the putative form of the planar Holant dichotomy. It was determined that a planar \#CSP${ }^{d}$ dichotomy in the putative form would be both a more modest, and thus hopefully more attainable, intermediate step as well as a good launch station for the final goal. However after some attempt, even the planar \#CSP${ }^{d}$ dichotomy appeared too difficult to achieve, and so we scaled back the ambition to prove just a planar \#CSP ${ }^{2}$ dichotomy. Luckily, a successful \#CSP ${ }^{2}$ dichotomy can carry most of the weight of a full \#CSP ${ }^{d}$ dichotomy, and, as it turned out, the putative form of the planar $\# \mathrm{CSP}^{2}$ dichotomy is true while that of planar \#CSP $^{d}$ is not. Ironically, many steps of our proof in this paper were guided by the 
putative form of the complexity classification. The discovery of the new tractable problems changed the original plan, but also helped complete the picture.

Coming back to the challenge of the P vs. NP question posed by Valiant's holographic algorithms, we venture the opinion that the dichotomy theorem provides one satisfactory answer. Indeed, it would be difficult to conceive a world where \#P is P, and yet all this algebraic theory can somehow maintain a consistent, sharp but faux division where there is none. (Consider the following Gedankenexperiment: \#P is really equal to $\mathrm{P}$, but Nature conspires against us and keeps scores on how much of \#P we have learned to be in P. For every problem in this broad class that is yet unknown to be in $\mathrm{P}$ we are allowed to prove it \#P-hard - a superfluous notion really, since every problem in \#P, being equal to $\mathrm{P}$, is \#P-hard. But for every problem in this broad class already known to be in $\mathrm{P}$, it makes sure that our proof for \#P-hardness on that problem fails, thus preventing us from making the ultimate discovery. This seems to us most implausible.)

After the preliminary version of the present paper [9] appeared in FOCS 2015, more progress has been made in the classification program of counting problems $[1,2,8,20,36]$. Ironically, if we go back to the \#CSP setting, then holographic algorithms with matchgates become universal again [8], despite the fact that it designed for the Holant setting. This generalizes the previous classification theorem [26] from symmetric constraint functions to general (not necessarily symmetric) constraint functions. Nevertheless, many problems are still left open, most of which relate to generalizing results in the current paper to asymmetric (i.e., not necessarily symmetric) signatures. For example, a \#CSP${ }^{2}$ dichotomy has been proved for asymmetric signatures (by combining results from [36] and [20]). But it is open in the planar setting. Also, classifying all Holant problems for general asymmetric complex-weighted signatures remains elusive. Partial results have been obtained for Holant $^{c}$ problems [2] or Holant problems with non-negatively weighted signatures [36].

\section{Preliminaries}

\subsection{Problems and Definitions}

The framework of Holant problems is defined for functions mapping any $[q]^{n} \rightarrow$ $R$ for a finite $q$ and some commutative semiring $R$. In this paper, we investigate complex-weighted Boolean Holant problems, that is, all functions are of the form $[2]^{n} \rightarrow \mathbb{C}$. For consideration of models of computation, functions take complex algebraic numbers.

Graphs may have self-loops and parallel edges. A graph without self-loops or parallel edges is a simple graph. Fix a set of local constraint functions $\mathcal{F}$. A signature grid $\Omega=(G, \pi)$ consists of a graph $G=(V, E)$, where $\pi$ assigns to each vertex $v \in V$ and its incident edges some $f_{v} \in \mathcal{F}$ and its input variables. We say that $\Omega$ is a planar signature grid if $G$ is planar, where the variables of $f_{v}$ are ordered counter- 
clockwise starting from an edge specified by $\pi$. The Holant problem on instance $\Omega$ is to evaluate $\operatorname{Holant}(\Omega ; \mathcal{F})=\sum_{\sigma} \prod_{v \in V} f_{v}\left(\left.\sigma\right|_{E(v)}\right)$, a sum over all edge assignments $\sigma: E \rightarrow\{0,1\}$, where $E(v)$ denotes the incident edges of $v$ and $\left.\sigma\right|_{E(v)}$ denotes the restriction of $\sigma$ to $E(v)$. We write $G$ in place of $\Omega$ when $\pi$ is clear from context.

A function $f_{v}$ can be represented by listing its values in lexicographical order as in a truth table, which is a vector in $\mathbb{C}^{2^{\operatorname{deg}(v)}}$, or as a tensor in $\left(\mathbb{C}^{2}\right)^{\otimes \operatorname{deg}(v)}$. A function $f \in \mathcal{F}$ is also called a signature. A symmetric signature $f$ on $n$ Boolean variables can be expressed as $\left[f_{0}, f_{1}, \ldots, f_{n}\right]$, where $f_{w}$ is the value of $f$ on inputs of Hamming weight $w$. An example is the EQUALITY signature $={ }_{n}$ of arity $n$, which is $[1,0, \ldots, 0,1]$ with $n-1$ zero entries.

In this paper, we prove complexity classifications for counting problems specified by symmetric signatures. A Holant problem is parametrized by a set of signatures.

Definition 2.1 Given a set of signatures $\mathcal{F}$, we define the counting problem $\operatorname{Holant}(\mathcal{F})$ as:

Input: A signature grid $\Omega=(G, \pi)$;

Output: $\operatorname{Holant}(\Omega ; \mathcal{F})$.

The problem Pl-Holant $(\mathcal{F})$ is defined similarly using a planar signature grid.

A signature $f$ of arity $n$ is degenerate if there exist unary signatures $u_{j} \in \mathbb{C}^{2}(1 \leq$ $j \leq n$ ) such that $f=u_{1} \otimes \cdots \otimes u_{n}$. A symmetric degenerate signature has the form $u^{\otimes n}$. Replacing such signatures by $n$ copies of the corresponding unary signature does not change the Holant value. Replacing a signature $f \in \mathcal{F}$ by a constant multiple $c f$, where $c \neq 0$, does not change the complexity of $\operatorname{Holant}(\mathcal{F})$. In this paper, we may say we obtain a signature $f$ when in fact we have obtained a signature $c f$ for some $c \neq 0$. It introduces a global nonzero factor $c^{n}$ to $\operatorname{Holant}(\Omega ; \mathcal{F})$, where $n$ is the number of occurrences of $c f$ in $\Omega$.

We allow $\mathcal{F}$ to be an infinite set. For Pl-Holant $(\mathcal{F})$ to be tractable, the problem must be computable in polynomial time even when the description of the signatures in the input $\Omega$ are included in the input size, where a local constraint function $f$ is specified by its signature entries. In contrast, we say $\mathrm{Pl}-\mathrm{Holant}(\mathcal{F})$ is \#P-hard if there exists a finite subset of $\mathcal{F}$ for which the problem is \#P-hard. We say a signature set $\mathcal{F}$ is tractable (resp. \#P-hard) if the corresponding counting problem $\mathrm{Pl}-\operatorname{Holant}(\mathcal{F})$ is tractable (resp. \#P-hard). Similarly for a signature $f$, we say $f$ is tractable (resp. \#Phard) if $\{f\}$ is. We denote polynomial time Turing reduction and equivalence by $\leq_{T}$ and $\equiv_{T}$ respectively.

\subsection{Holographic Reduction}

To introduce the idea of holographic reductions, it is convenient to consider bipartite graphs. For a general graph, we can always transform it into a bipartite graph while preserving the Holant value, as follows. For each edge in the graph, we replace it by a path of length two. (This operation is called the 2-stretch of the graph and yields 
the edge-vertex incidence graph.) Each new vertex is assigned the binary EQUALITY signature $(=2)=[1,0,1]$.

We use Holant $(\mathcal{F} \mid \mathcal{G})$ to denote the Holant problem over signature grids with a bipartite graph $H=(U, V, E)$, where each vertex in $U$ or $V$ is assigned a signature in $\mathcal{F}$ or $\mathcal{G}$, respectively. Signatures in $\mathcal{F}$ are considered as row vectors (or covariant tensors); signatures in $\mathcal{G}$ are considered as column vectors (or contravariant tensors) [22]. Similarly, Pl-Holant $(\mathcal{F} \mid \mathcal{G})$ denotes the Holant problem over signature grids with a planar bipartite graph.

For a 2-by-2 matrix $T$ and a signature set $\mathcal{F}$, define $T \mathcal{F}=\{g \mid \exists f \in \mathcal{F}$ of arity $\left.n, g=T^{\otimes n} f\right\}$, and similarly for $\mathcal{F} T$. Whenever we write $T^{\otimes n} f$ or $T \mathcal{F}$, we view the signatures as column vectors; similarly for $f T^{\otimes n}$ or $\mathcal{F} T$ as row vectors. In the special case that $T=\left[\begin{array}{ll}1 & 1 \\ 1 & -1\end{array}\right]$, we also define $T \mathcal{F}=\widehat{\mathcal{F}}$.

Let $T$ be an invertible 2-by-2 matrix. The holographic transformation defined by $T$ is the following operation: given a signature grid $\Omega=(H, \pi)$ of Holant $(\mathcal{F} \mid \mathcal{G})$, for the same bipartite graph $H$, we get a new grid $\Omega^{\prime}=\left(H, \pi^{\prime}\right)$ of Holant $\left(\mathcal{F} T \mid T^{-1} \mathcal{G}\right)$ by replacing each signature in $\mathcal{F}$ or $\mathcal{G}$ with the corresponding signature in $\mathcal{F} T$ or $T^{-1} \mathcal{G}$.

Theorem 2.2 (Valiant's Holant Theorem [45]) If $T \in \mathbb{C}^{2 \times 2}$ is an invertible matrix, then we have $\operatorname{Holant}(\Omega ; \mathcal{F} \mid \mathcal{G})=\operatorname{Holant}\left(\Omega^{\prime} ; \mathcal{F} T \mid T^{-1} \mathcal{G}\right)$.

Therefore, an invertible holographic transformation does not change the complexity of the Holant problem in the bipartite setting. Furthermore, there is a special kind of holographic transformations, the orthogonal transformations, that preserve the binary equality and thus can be used freely in the standard setting.

Theorem 2.3 (Theorem 2.6 in [17]) If $T \in \mathbf{O}_{2}(\mathbb{C})$ is an orthogonal matrix (i.e. $\left.T T^{T}=I_{2}\right)$, then $\operatorname{Holant}(\Omega ; \mathcal{F})=\operatorname{Holant}\left(\Omega^{\prime} ; T \mathcal{F}\right)$.

We frequently apply a holographic transformation defined by the matrix $Z=$ $\frac{1}{\sqrt{2}}\left[\begin{array}{ll}1 & 1 \\ i & -i\end{array}\right]$ (or sometimes without the nonzero factor of $\frac{1}{\sqrt{2}}$ since this does not affect the complexity). This matrix has the property that the binary EQUALITY signature $(=2)=[1,0,1]$ is transformed to $[1,0,1] Z^{\otimes 2}=[0,1,0]=\left(\neq_{2}\right)$, the binary DISEQUALITY signature.

An important definition involving a holographic transformation is the notion of a signature set being transformable.

Definition 2.4 We say a signature set $\mathcal{F}$ is $\mathscr{C}$-transformable if there exists a $T \in$ $\mathbf{G L}_{2}(\mathbb{C})$ such that $[1,0,1] T^{\otimes 2} \in \mathscr{C}$ and $\mathcal{F} \subseteq T \mathscr{C}$.

This definition is important because if Pl-Holant $(\mathscr{C})$ is tractable, then $\operatorname{Pl}$-Holant $(\mathcal{F})$ is tractable for any $\mathscr{C}$-transformable set $\mathcal{F}$. 


\subsection{Counting Constraint Satisfaction Problems}

We can define the framework of counting constraint satisfaction problems (\#CSP) in terms of the Holant framework. An instance of $\# \operatorname{CSP}(\mathcal{F})$ has the following bipartite view. Create a vertex for each variable and each constraint. Connect a variable vertex to a constraint vertex if the variable appears in the constraint. This bipartite graph is also known as the constraint graph. Each variable can be viewed as an EQUALITY function. In this way we obtain a signature grid. A variable can take values 0 or 1 , and the EQUALITY function forces all incident edges to take the same value. Under this view, we see that $\# \operatorname{CSP}(\mathcal{F}) \equiv_{T}$ Holant $(\mathcal{E} \mathcal{Q} \mid \mathcal{F})$, where $\mathcal{E} \mathcal{Q}=\left\{={ }_{1},=_{2},=_{3}, \ldots\right\}$ is the set of EQUALITY signatures of all arities. By restricting to planar constraint graphs, we have the planar \#CSP framework, which we denote by Pl-\#CSP. The construction above also shows that $\operatorname{Pl}-\# \operatorname{CSP}(\mathcal{F}) \equiv_{T} \operatorname{Pl}$-Holant $(\mathcal{E} \mathcal{Q} \mid \mathcal{F})$.

For any positive integer $d$, the problem $\# \operatorname{CSP}^{d}(\mathcal{F})$ is the same as $\# \operatorname{CSP}(\mathcal{F})$ except that every variable appears a multiple of $d$ times. Thus, $\operatorname{Pl}_{-\# C S P^{d}}(\mathcal{F}) \equiv_{T}$ Pl-Holant $\left(\mathcal{E} \mathcal{Q}_{d} \mid \mathcal{F}\right)$, where $\mathcal{E} \mathcal{Q}_{d}=\left\{={ }_{d},=2 d,={ }_{3 d}, \ldots\right\}$ is the set of EQUALITY signatures of arities that are a multiple of $d$. If $d \in\{1,2\}$, then we further have

$$
\operatorname{Pl}-\# \operatorname{CSP}^{d}(\mathcal{F}) \equiv_{T} \operatorname{Pl} \text {-Holant }\left(\mathcal{E} \mathcal{Q}_{d} \mid \mathcal{F}\right) \equiv_{T} \operatorname{Pl} \text {-Holant }\left(\mathcal{E} \mathcal{Q}_{d} \cup \mathcal{F}\right) \text {. }
$$

For the second equivalence the reduction from left to right is trivial. For the other direction, we take a signature grid for the problem on the right and create a bipartite signature grid for the problem on the left such that both signature grids have the same Holant value up to an easily computable factor. If two signatures in $\mathcal{F}$ are assigned to adjacent vertices, then we subdivide all edges between them and assign the binary EQUALITY signature $=_{2} \in \mathcal{E} \mathcal{Q}_{d}$ to all new vertices. Suppose EQUALITY signatures $={ }_{n},{ }_{m} \in \mathcal{E} \mathcal{Q}_{d}$ are assigned to adjacent vertices connected by $k$ edges. If $n=m=k$, then we simply remove these two vertices. The Holant of the resulting signature grid differs from the original by a factor of 2 . Otherwise, we contract all $k$ edges and assign ${ }_{n+m-2 k} \in \mathcal{E} \mathcal{Q}_{d}$ to the new vertex.

\subsection{Realization}

One basic notion used throughout the paper is realization. We say a signature $f$ is realizable or constructible from a signature set $\mathcal{F}$ if there is a gadget with some dangling edges such that each vertex is assigned a signature from $\mathcal{F}$, and the resulting graph, when viewed as a black-box signature with inputs on the dangling edges, is exactly $f$. If $f$ is realizable from a set $\mathcal{F}$, then we can freely add $f$ into $\mathcal{F}$ while preserving the complexity. (Often it is convenient to ignore a nonzero constant factor for the signature realized by a gadget construction; however at other times it is more convenient to keep the exact value so that claims of a particular construction can be more readily verified numerically.)

Formally, such a notion is defined by an $\mathcal{F}$-gate [19]. An $\mathcal{F}$-gate is similar to a signature grid $(G, \pi)$ for Holant $(\mathcal{F})$ except that $G=(V, E, D)$ is a graph with some dangling edges $D$. The dangling edges define external variables for the $\mathcal{F}$-gate. (See 
Fig. 1 for an example.) We denote the regular edges in $E$ by $1,2, \ldots, m$ and the dangling edges in $D$ by $m+1, \ldots, m+n$. Then we can define a function $\Gamma$ for this $\mathcal{F}$-gate as

$$
\Gamma\left(y_{1}, \ldots, y_{n}\right)=\sum_{x_{1}, \ldots, x_{m} \in\{0,1\}} H\left(x_{1}, \ldots, x_{m}, y_{1}, \ldots, y_{n}\right)
$$

where $\left(y_{1}, \ldots, y_{n}\right) \in\{0,1\}^{n}$ is an assignment on the dangling edges and $H\left(x_{1}, \ldots, x_{m}, y_{1}, \ldots, y_{n}\right)$ is the value of the signature grid on an assignment of all edges in $G$, which is the product of evaluations at all internal vertices. We also call this function $\Gamma$ the signature of the $\mathcal{F}$-gate.

An $\mathcal{F}$-gate is planar if the underlying graph $G$ is a planar graph, and the dangling edges, ordered counterclockwise corresponding to the order of the input variables, are in the outer face in a planar embedding. A planar $\mathcal{F}$-gate can be used in a planar signature grid as if it is just a single vertex with the particular signature.

Using the idea of planar $\mathcal{F}$-gates, we can reduce one planar Holant problem to another. Suppose $g$ is the signature of some planar $\mathcal{F}$-gate. Then $\operatorname{Pl}$-Holant $(\mathcal{F} \cup$ $\{g\}) \leq_{T}$ Pl-Holant $(\mathcal{F})$. The reduction is simple. Given an instance of $\mathrm{Pl}-\operatorname{Holant}(\mathcal{F} \cup$ $\{g\}$ ), by replacing every appearance of $g$ by the $\mathcal{F}$-gate, we get an instance of Pl-Holant $(\mathcal{F})$. Since the signature of the $\mathcal{F}$-gate is $g$, the Holant values for these two signature grids are identical.

Although our main result is about symmetric signatures, some of our proofs utilize asymmetric signatures. When a gadget has an asymmetric signature, we indicate the ordering of the variables by placing a hollow diamond on the edge corresponding to the first input in the figure. The remaining inputs are ordered counterclockwise around the vertex.

We note that even for a very simple signature set $\mathcal{F}$, the signatures for all $\mathcal{F}$-gates can be quite complicated and expressive.

Fig. 1 An $\mathcal{F}$-gate with 5 dangling edges

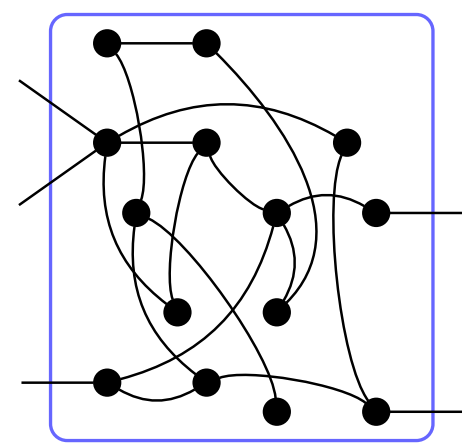




\subsection{Tractable Signature Sets}

We define the sets of signatures that were previously known to be tractable. All quotations of results and definitions from $[11,12,26]$, both in this section and throughout the paper, refer to the full versions of these papers.

\section{Affine Signatures}

Definition 2.5 (Definition 3.1 in [18]) A $k$-ary function $f\left(x_{1}, \ldots, x_{k}\right)$ is affine if it has the form

$$
\lambda \cdot \chi_{A x=\mathbf{b}} \cdot i^{\sum_{j=1}^{n}\left\langle\mathbf{v}_{j}, x\right\rangle},
$$

where $\lambda \in \mathbb{C}, A$ is a matrix over the two-element finite field $\mathbb{F}_{2}, \mathbf{b}$ and $\mathbf{v}_{j}$ are vectors over $\mathbb{F}_{2}, x=\left(x_{1}, x_{2}, \ldots, x_{k}\right)^{T}$, and $\chi$ is a $0-1$ indicator function such that $\chi_{A x=\mathbf{b}}$ is 1 iff $A x=\mathbf{b}$. Note that the dot product $\left\langle\mathbf{v}_{j}, x\right\rangle$ is calculated over $\mathbb{F}_{2}$ with output in $\{0,1\}$, and the summation $\sum_{j=1}^{n}$ on the exponent of $i=\sqrt{-1}$ is evaluated as a sum $\bmod 4$ of $0-1$ terms. We use $\mathscr{A}$ to denote the set of all affine functions.

Notice that there is no restriction on the number of rows in the matrix $A$. It is permissible that $A$ is the zero matrix so that $\chi_{A x=0}=1$ holds for all $x$. An equivalent way to express the exponent of $i$ is as a quadratic polynomial where all cross terms have an even coefficient (cf. [5]).

It is known that the set of non-degenerate symmetric signatures in $\mathscr{A}$ is precisely the nonzero signatures $\left(\lambda \neq 0\right.$ in the expressions below) in $\mathscr{F}_{1} \cup \mathscr{F}_{2} \cup \mathscr{F}_{3}$ with arity at least 2 , where $\mathscr{F}_{1}, \mathscr{F}_{2}$, and $\mathscr{F}_{3}$ are three families of signatures defined as

$$
\begin{aligned}
& \mathscr{F}_{1}=\left\{\lambda\left([1,0]^{\otimes k}+i^{r}[0,1]^{\otimes k}\right) \mid \lambda \in \mathbb{C}, k=1,2, \ldots, r=0,1,2,3\right\}, \\
& \mathscr{F}_{2}=\left\{\lambda\left([1,1]^{\otimes k}+i^{r}[1,-1]^{\otimes k}\right) \mid \lambda \in \mathbb{C}, k=1,2, \ldots, r=0,1,2,3\right\}, \text { and } \\
& \mathscr{F}_{3}=\left\{\lambda\left([1, i]^{\otimes k}+i^{r}[1,-i]^{\otimes k}\right) \mid \lambda \in \mathbb{C}, k=1,2, \ldots, r=0,1,2,3\right\} .
\end{aligned}
$$

We explicitly list these signatures up to an arbitrary constant multiple from $\mathbb{C}$ :
1. $[1,0, \ldots, 0, \pm 1]$
$\left(\mathscr{F}_{1}, r=0,2\right)$
2. $[1,0, \ldots, 0, \pm i]$;
$\left(\mathscr{F}_{1}, r=1,3\right)$
3. $[1,0,1,0, \ldots, 0$ or 1$]$;
$\left(\mathscr{F}_{2}, r=0\right)$
4. $[1,-i, 1,-i, \ldots,(-i)$ or 1$]$;
$\left(\mathscr{F}_{2}, r=1\right)$
5. $[0,1,0,1, \ldots, 0$ or 1$]$;
$\left(\mathscr{F}_{2}, r=2\right)$
6. $[1, i, 1, i, \ldots, i$ or 1$]$;
$\left(\mathscr{F}_{2}, r=3\right)$
7. $[1,0,-1,0,1,0,-1,0, \ldots, 0$ or 1 or $(-1)]$;
$\left(\mathscr{F}_{3}, r=0\right)$
8. $[1,1,-1,-1,1,1,-1,-1, \ldots, 1$ or $(-1)]$;
$\left(\mathscr{F}_{3}, r=1\right)$
9. $[0,1,0,-1,0,1,0,-1, \ldots, 0$ or 1 or $(-1)]$;
$\left(\mathscr{F}_{3}, r=2\right)$
10. $[1,-1,-1,1,1,-1,-1,1, \ldots, 1$ or $(-1)]$.
$\left(\mathscr{F}_{3}, r=3\right)$ 


\section{Product-Type Signatures}

Definition 2.6 (Definition 3.3 in [18]) A function is of product type if it can be expressed as a product of unary functions, binary equality functions $([1,0,1])$, and binary disequality functions $([0,1,0])$. We use $\mathscr{P}$ to denote the set of product-type functions.

For a function $f \in \mathscr{P}$ of arity $n$, since the product of unary, binary equality, and binary disequality functions in its definition can be on separate and overlapping choices of subsets of $n$ variables, $f$ in general is not symmetric. For example, $\mathscr{P}$ contains weighted binary disequality functions $f(x, y)$, where $f(0,0)=f(1,1)=0$, $f(0,1)=a$, and $f(1,0)=b$. We denote this by its truth table $(0, a, b, 0)$. An alternate definition for $\mathscr{P}$, implicit in [21], is the tensor closure of signatures with support on two complementary bit vectors. It can be shown (see [27, Lemma 2.2]) that if $f$ is a symmetric signature in $\mathscr{P}$, then $f$ is either degenerate, binary DISEQUALITY $\neq_{2}$, or $[a, 0, \ldots, 0, b]$ for some $a, b \in \mathbb{C}$.

\section{Matchgate Signatures}

Matchgates were introduced by Valiant $[42,43]$ to give polynomial-time algorithms for a collection of counting problems over planar graphs. As the name suggests, problems expressible by matchgates can be reduced to computing a weighted sum of perfect matchings. The latter problem is tractable over planar graphs by Kasteleyn's algorithm [30], a.k.a. the FKT algorithm [29, 41]. These counting problems are naturally expressed in the Holant framework using matchgate signatures. We use $\mathscr{M}$ to denote the set of all matchgate signatures; thus Pl-Holant $(\mathscr{M})$ is tractable. The function $[0,1,0, \ldots, 0]$ of arity $k$ belongs to $\mathscr{M}$, and is called the EXACTONE func- $^{-}$ tion. More generally, we can define EXACTONE $k: a_{1}, \ldots, a_{k}$ as a weighted version of EXACTONE $k$, which takes value $a_{i}$ if the input has Hamming weight 1 and $x_{i}=1$; the function outputs 0 on all other inputs. This function also belongs to $\mathscr{M}$.

Holographic transformations extend the reach of the FKT algorithm even further, as stated below.

Theorem 2.7 Let $\mathcal{F}$ be any set of complex-valued signatures in Boolean variables. If $\mathcal{F}$ is $\mathscr{M}$-transformable, then $\mathrm{Pl}-\operatorname{Holant}(\mathcal{F})$ is computable in polynomial time.

Matchgate signatures are characterized by the matchgate identities (see [10] for the identities and a self-contained proof). The parity of a matchgate signature is even (resp. odd) if its support is on entries of even (resp. odd) Hamming weight. We explicitly list all the symmetric signatures in $\mathscr{M}$ (see [10]).

Proposition 2.8 Let $f$ be a symmetric signature in $\mathscr{M}$. Then there exist $a, b \in \mathbb{C}$ and $n \in \mathbb{N}$ such that $f$ takes one of the following forms:
1. $\left[a^{n}, 0, a^{n-1} b, 0, \ldots, 0, a b^{n-1}, 0, b^{n}\right]$
2. $\left[a^{n}, 0, a^{n-1} b, 0, \ldots, 0, a b^{n-1}, 0, b^{n}, 0\right]$
(of arity $2 n \quad \geq 2$ );
3. $\left[0, a^{n}, 0, a^{n-1} b, 0, \ldots, 0, a b^{n-1}, 0, b^{n}\right]$
(of arity $2 n+1 \geq 1$ );
4. $\left[0, a^{n}, 0, a^{n-1} b, 0, \ldots, 0, a b^{n-1}, 0, b^{n}, 0\right]$
(of arity $2 n+1 \geq 1)$;
(of arity $2 n+2 \geq 2$ ). 
In the last three cases with $n=0$, the signatures are [1, 0], [0, 1], and [0, 1, 0]. Any multiple of these is also a matchgate signature.

Roughly speaking, the symmetric matchgate signatures have 0 for every other entry (which is called the parity condition), and form a geometric progression with the remaining entries.

Another useful way to view the symmetric signature in $\mathscr{M}$ is via a low tensor rank decomposition. To state these low rank decompositions, we use the following definition.

Definition 2.9 Let $S_{n}$ be the symmetric group of degree $n$. Then for positive integers $t$ and $n$ with $t \leq n$ and unary signatures $v, v_{1}, \ldots, v_{n-t}$, we define

$$
\operatorname{Sym}_{n}^{t}\left(v ; v_{1}, \ldots, v_{n-t}\right)=\sum_{\pi \in S_{n}} \bigotimes_{k=1}^{n} u_{\pi(k)},
$$

where the ordered sequence $\left(u_{1}, u_{2}, \ldots, u_{n}\right)=(\underbrace{v, \ldots, v}_{t \text { copies }}, v_{1}, \ldots, v_{n-t})$.

Proposition 2.10 Let $f$ be a symmetric signature in $\mathscr{M}$ of arity $n$. Then there exist $a, b, \lambda \in \mathbb{C}$ such that $f$ takes one of the following forms:

1. $[a, b]^{\otimes n}+[a,-b]^{\otimes n}= \begin{cases}2\left[a^{n}, 0, a^{n-2} b^{2}, 0, \ldots, 0, b^{n}\right] & n \text { is even, } \\ 2\left[a^{n}, 0, a^{n-2} b^{2}, 0, \ldots, 0, a b^{n-1}, 0\right] & n \text { is odd } ;\end{cases}$

2. $[a, b]^{\otimes n}-[a,-b]^{\otimes n}= \begin{cases}2\left[0, a^{n-1} b, 0, a^{n-3} b^{3}, 0, \ldots, 0, a b^{n-1}, 0\right] & n \text { is even, } \\ 2\left[0, a^{n-1} b, 0, a^{n-3} b^{3}, 0, \ldots, 0, b^{n}\right] & n \text { is odd; }\end{cases}$

3. $\lambda \operatorname{Sym}_{n}^{n-1}([1,0] ;[0,1])=[0, \lambda, 0, \ldots, 0]$;

4. $\lambda \operatorname{Sym}_{n}^{n-1}([0,1] ;[1,0])=[0, \ldots, 0, \lambda, 0]$.

While the symmetric signature notation such as $[0, \lambda, 0, \ldots, 0]$ is intuitive, the notation of tensor powers and $\operatorname{Sym}_{n}^{t}(-;-)$ will be convenient for holographic transformations.

The understanding of matchgates was further developed in [16], which characterized, for every symmetric signature, the set of holographic transformations under which the transformed signature becomes a matchgate signature.

\section{Vanishing Signatures}

Vanishing signatures were first introduced in [25] in the parity setting to denote signatures for which the Holant value is always 0 modulo 2.

Definition 2.11 A set of signatures $\mathcal{F}$ is called vanishing if the value $\operatorname{Holant}_{\Omega}(\mathcal{F})$ is 0 for every signature grid $\Omega$. A signature $f$ is called vanishing if the singleton set $\{f\}$ is vanishing.

A Holant problem defined only by vanishing signatures is trivially tractable by definition. The question is how to determine which sets of signatures are vanishing? We introduced the following definitions to answer this question. 
Definition 2.12 (Definition 17, p. 1683 in [11]) A nonzero symmetric signature $f$ of arity $n$ has positive vanishing degree $k \geq 1$, which is denoted by $\mathrm{vd}^{+}(f)=k$, if $k \leq n$ is the largest positive integer such that there exists $n-k$ unary signatures $v_{1}, \ldots, v_{n-k}$ satisfying

$$
f=\operatorname{Sym}_{n}^{k}\left([1, i] ; v_{1}, \ldots, v_{n-k}\right) .
$$

If $f$ cannot be expressed as such a symmetrization form, we define $\mathrm{vd}^{+}(f)=0$. If $f$ is the all zero signature, define $\mathrm{vd}^{+}(f)=n+1$.

We define negative vanishing degree $\mathrm{vd}^{-}$similarly, using $-i$ instead of $i$.

Definition 2.13 (Definition 18, p. 1684 in [11]) For $\sigma \in\{+,-\}$, we define $\mathscr{V}^{\sigma}=$ $\left\{f \mid 2 \mathrm{vd}^{\sigma}(f)>\operatorname{arity}(f)\right\}$.

Furthermore, we let $\mathscr{V}=\mathscr{V}^{+} \cup \mathscr{V}^{-}$. The fact that $\mathscr{V}$ is closed under orthogonal transformations follows directly from the next lemma.

Lemma 2.14 (Lemma 29, p.1690 in [11]) For a symmetric signature $f$ of arity $n$, $\sigma \in\{+,-\}$, and an orthogonal matrix $T \in \mathbb{C}^{2 \times 2}, \mathrm{vd}^{\sigma}(f)$ is either $\mathrm{vd}^{\sigma}\left(T^{\otimes n} f\right)$ or $\operatorname{vd}^{-\sigma}\left(T^{\otimes n} f\right)$.

The following characterization of vanishing signature sets holds.

Theorem 2.15 (Theorem 26, p. 1689 in [11]) Let $\mathcal{F}$ be a set of symmetric signatures. Then $\mathcal{F}$ is vanishing if and only if $\mathcal{F} \subseteq \mathscr{V}^{+}$or $\mathcal{F} \subseteq \mathscr{V}^{-}$.

To prove this theorem, two more definitions were made, which complement the previous two definitions because of Corollary 2.18.

Definition 2.16 (Definition 20, p. 1684 in [11]) A symmetric signature $f=$ $\left[f_{0}, f_{1}, \ldots, f_{n}\right]$ of arity $n$ is in $\mathscr{R}_{t}^{+}$for a nonnegative integer $t \geq 0$ if $t>n$ or for any $0 \leq k \leq n-t, f_{k}, \ldots, f_{k+t}$ satisfy the recurrence relation

$$
\left(\begin{array}{l}
t \\
t
\end{array}\right) i^{t} f_{k+t}+\left(\begin{array}{c}
t \\
t-1
\end{array}\right) i^{t-1} f_{k+t-1}+\cdots+\left(\begin{array}{l}
t \\
0
\end{array}\right) i^{0} f_{k}=0 .
$$

We define $\mathscr{R}_{t}^{-}$similarly but with $-i$ in place of $i$ in (2.3).

Definition 2.17 (Definition 21, p. 1684 in [11]) For a nonzero symmetric signature $f$ of arity $n$, it is of positive (resp. negative) recurrence degree $t \leq n$, denoted by $\mathrm{rd}^{+}(f)=t$ (resp. $\left.\mathrm{rd}^{-}(f)=t\right)$, if and only if $f \in \mathscr{R}_{t+1}^{+}-\mathscr{R}_{t}^{+}$(resp. $f \in \mathscr{R}_{t+1}^{-}-$ $\left.\mathscr{R}_{t}^{-}\right)$. If $f$ is the all zero signature, we define $\operatorname{rd}^{+}(f)=\operatorname{rd}^{-}(f)=-1$.

Corollary 2.18 (Corollary 23, p. 1686 in [11]) If $f$ is a symmetric signature and $\sigma \in\{+,-\}$, then $\operatorname{vd}^{\sigma}(f)+\operatorname{rd}^{\sigma}(f)=\operatorname{arity}(f)$.

The following lemma gives a simple formula for the recurrence degree of a signature when expressed under the $Z$ transformation. 
Lemma 2.19 (Lemma 30, p. 1690 of [11]) Suppose $f$ is a symmetric signature of arity $n$. Let $\hat{f}=\left(Z^{-1}\right)^{\otimes n} f$. If $\mathrm{rd}^{+}(f)=d$, then $\hat{f}=\left[\hat{f_{0}}, \hat{f}_{1}, \ldots, \hat{f}_{d}, 0, \ldots, 0\right]$ and $\hat{f}_{d} \neq 0$. Also $f \in \mathscr{R}_{d}^{+}$iff all nonzero entries of $\hat{f}$ are among the first $d$ entries in its symmetric signature notation.

Similarly, if $\mathrm{rd}^{-}(f)=d$, then $\hat{f}=\left[0, \ldots, 0, \hat{f}_{n-d}, \ldots, \hat{f}_{n}\right]$ and $\hat{f}_{n-d} \neq 0$. Also $f \in \mathscr{R}_{d}^{-}$iff all nonzero entries of $\hat{f}$ are among the last $d$ entries in its symmetric signature notation.

The following lemma is a reduction involving binary signatures in the $Z$ basis. It is used in Section 4 to help determine what binary signatures can mix with vanishing signatures. The original statement is for general graphs, but the proof clearly holds for planar graphs as well.

Lemma 2.20 (Lemma 65, p. 1724 in [11]) Let $x \in \mathbb{C}$. If $x \neq 0$, then for any set $\mathcal{F}$ containing $[x, 1,0]$, we have

$$
\text { Pl-Holant }\left(\neq_{2} \mid \mathcal{F} \cup\{[v, 1,0]\}\right) \leq_{T} \text { Pl-Holant }\left(\neq_{2} \mid \mathcal{F}\right)
$$

for any $v \in \mathbb{C}$.

\subsection{Some Known Dichotomies}

Here we list several known dichotomies. The first is the dichotomy for Holant.

Theorem 2.21 (Theorem 31, p. 1691 in [11]) Let $\mathcal{F}$ be any set of symmetric, complex-valued signatures in Boolean variables. Then Holant $(\mathcal{F})$ is \#P-hard unless $\mathcal{F}$ satisfies one of the following conditions, in which case the problem is in II:

1. All non-degenerate signatures in $\mathcal{F}$ are of arity at most 2;

2. $\mathcal{F}$ is $\mathscr{A}$-transformable;

3. $\mathcal{F}$ is $\mathscr{P}$-transformable;

4. $\mathcal{F} \subseteq \mathscr{V}^{\sigma} \cup\left\{f \in \mathscr{R}_{2}^{\sigma} \mid \operatorname{arity}(f)=2\right\}$ for $\sigma \in\{+,-\}$;

5. All non-degenerate signatures in $\mathcal{F}$ are in $\mathscr{R}_{2}^{\sigma}$ for $\sigma \in\{+,-\}$.

We also use several dichotomy theorems for planar Holant problems with additional restrictions. The first of these is a dichotomy theorem for a single signature of small arity. It is a combination of Theorem 6.1 (p. 882) in [19] and Theorem 5.6 (p. 16) in [26] for arity 3 and 4, respectively. This theorem forms the base case of an inductive proof of Theorem 6.1, our single signature dichotomy.

Theorem 2.22 If $f$ is a non-degenerate, symmetric, complex-valued signature of arity 3 or 4 in Boolean variables, then Pl-Holant $(f)$ is \#P-hard unless $f$ satisfies one of the following conditions, in which case, the problem is computable in polynomial time:

1. Holant $(f)$ is tractable (i.e. $f$ is $\mathscr{A}$-transformable, $\mathscr{P}$-transformable, or vanishing);

2. $f$ is $\mathscr{M}$-transformable. 
We also state a corollary of this result, which shows that counting weighted matchings in 4-regular planar graphs is \#P-hard. This is easier to apply than Theorem 2.22.

Corollary 2.23 (Lemma 5.5 in [26]) Let $v \in \mathbb{C}$. If $v \neq 0$, then Pl-Holant $([v, 1,0,0,0])$ is \#P-hard.

Next is a dichotomy theorem about counting complex weighted graph homomorphisms over degree prescribed graphs. This is essentially equivalent to Theorem 3 in [13]. A more explicitly equivalent statement is stated as Theorem 22 in [31].

Theorem 2.24 Let $S \subseteq \mathbb{Z}^{+}$containing some $r \geq 3$, let $\mathcal{G}=\left\{={ }_{k} \mid k \in S\right\}$, and let $d=\operatorname{gcd}(S)$. Further suppose that $f_{0}, f_{1}, f_{2} \in \mathbb{C}$. Then Pl-Holant $\left(\left[f_{0}, f_{1}, f_{2}\right] \mid \mathcal{G}\right)$ is polynomial time computable in the following cases:

1. $f_{0} f_{2}=f_{1}^{2}$;

2. $f_{0}=f_{2}=0$;

3. $f_{1}=0$;

4. $f_{0} f_{2}=-f_{1}^{2}$ and $f_{0}^{d}=-f_{2}^{d} \neq 0$;

5. $f_{0}^{d}=f_{2}^{d} \neq 0$.

In all other cases the problem is \#P-hard.

Theorem 2.24 is stated in an explicit form that is easy to apply. Conceptually, it can be restated as Theorem $2.24^{\prime}$, which supports the putative form of the Pl-\#CSP ${ }^{d}$ dichotomy. We denote by $\widehat{\mathscr{M}}=H \mathscr{M}$ the set of Matchgate signatures $\mathscr{M}$ transformed by the Hadamard basis $H=\left[\begin{array}{ll}1 & 1 \\ 1 & -1\end{array}\right]$.

Let $\mathcal{T}_{k}=\left\{\left[\begin{array}{ll}1 & 0 \\ 0 & \theta\end{array}\right] \mid \theta^{k}=1\right\}$ be a set of diagonal matrices of order dividing $k$.

Theorem 2.24' Let $S \subseteq \mathbb{Z}^{+}$contain $k \geq 3$, let $\mathcal{G}=\left\{={ }_{k} \mid k \in S\right\}$, and let $d=\operatorname{gcd}(S)$. Further suppose that $f$ is a non-degenerate, symmetric, complex-valued binary signature in Boolean variables. Then Pl-Holant $(f \mid \mathcal{G})$ is \#P-hard unless $f$ satisfies one of the following conditions, in which case, the problem is computable in polynomial time:

1. there exists $T \in \mathcal{T}_{4 d}$ such that $T^{\otimes 2} f \in \mathscr{A}$;

2. $f \in \mathscr{P}$;

3. there exists $T \in \mathcal{T}_{2 d}$ such that $T^{\otimes 2} f \in \widehat{\mathscr{M}}$.

Lastly, we quote the Pl-\#CSP dichotomy for symmetric signatures. It also supports the putative form of a dichotomy, which states that holographic algorithms using matchgates followed by the FKT algorithm is a universal strategy. 
Theorem 2.25 (Theorem 9.3 in [26]) Let $\mathcal{F}$ be any set of symmetric, complex-valued signatures in Boolean variables. Then $\mathrm{Pl}-\# \operatorname{CSP}(\mathcal{F})$ is \#P-hard unless $\mathcal{F} \subseteq \mathscr{A}, \mathcal{F} \subseteq$ $\mathscr{P}$, or $\mathcal{F} \subseteq \widehat{\mathscr{M}}$, in which case the problem is computable in polynomial time.

\subsection{Redundant Signature Matrices and Related Hardness Results}

Definition 2.26 (Definition 32, p. 1692 in [11]) A 4-by-4 matrix is redundant if its middle two rows and middle two columns are the same.

An example of a redundant matrix is the signature matrix of a symmetric arity 4 signature.

Definition 2.27 (Definition 33, p. 1693 in [11]) The signature matrix of a symmetric arity 4 signature $f=\left[f_{0}, f_{1}, f_{2}, f_{3}, f_{4}\right]$ is

$$
M_{f}=\left[\begin{array}{llll}
f_{0} & f_{1} & f_{1} & f_{2} \\
f_{1} & f_{2} & f_{2} & f_{3} \\
f_{1} & f_{2} & f_{2} & f_{3} \\
f_{2} & f_{3} & f_{3} & f_{4}
\end{array}\right] .
$$

This definition extends to an asymmetric signature $g$ as

$$
M_{g}=\left[\begin{array}{llll}
g^{0000} & g^{0010} & g^{0001} & g^{0011} \\
g^{0100} & g^{0110} & g^{0101} & g^{0111} \\
g^{1000} & g^{1010} & g^{1001} & g^{1011} \\
g^{1100} & g^{1110} & g^{1101} & g^{1111}
\end{array}\right],
$$

where $g^{w x y z}$ is the output of $g$ on input $w x y z$. When we present $g$ as an $\mathcal{F}$-gate, we order the four external edges ABCD counterclockwise. In $M_{g}$, the row index bits are ordered $\mathrm{AB}$ and the column index bits are ordered $\mathrm{DC}$, in the reverse order. We order them in this way so that when we link two arity $4 \mathcal{F}$-gates with signature matrices $A$ and $B$, the resulting signature matrix is the matrix product $A B$.

If $M_{g}$ is redundant, we also define the compressed signature matrix of $g$ in $\mathbb{C}^{3 \times 3}$ as

$$
\widetilde{M}_{g}=\left[\begin{array}{llll}
1 & 0 & 0 & 0 \\
0 & \frac{1}{2} & \frac{1}{2} & 0 \\
0 & 0 & 0 & 1
\end{array}\right] M_{g}\left[\begin{array}{lll}
1 & 0 & 0 \\
0 & 1 & 0 \\
0 & 1 & 0 \\
0 & 0 & 1
\end{array}\right] .
$$

Lemma 2.28 (Corollary 3.8 in [26]) Let $f$ be an arity 4 signature with complex weights. If $M_{f}$ is redundant and $\widetilde{M_{f}}$ is nonsingular, then Pl-Holant $(f)$ is \#P-hard.

Furthermore, by combining Lemma 2.28 with Lemma 39 (p. 1702) in [11], we obtain the planar version of Corollary 40 (p. 1703) in [11]. 

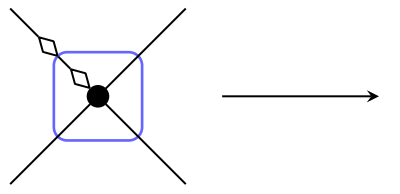

(a)

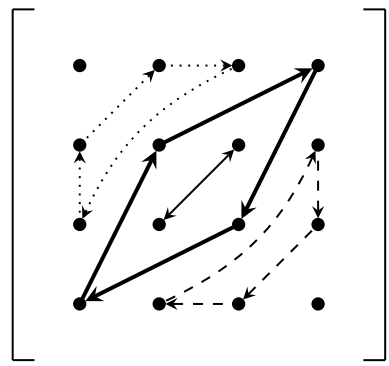

(b)

Fig. 2 The movement of the entries in the signature matrix of a quaternary signature under a counterclockwise rotation of the input edges. Entries of Hamming weight 1 are in the dotted cycle, entries of Hamming weight 2 are in the two solid cycles (one has length 4 and the other one is a swap), and entries of Hamming weight 3 are in the dashed cycle. a A counterclockwise rotation: Hollow diamonds inside and outside the box mark the first variable before and after the rotation. Other variables are cyclically ordered. b Movement of signature matrix entries under this rotation.

Corollary 2.29 Let $f$ be an arity 4 signature with complex weights. If there exists a nonsingular matrix $T \in \mathbb{C}^{2 \times 2}$ such that $\hat{f}=T^{\otimes 4} f$, where $M_{\hat{f}}$ is redundant and $\widetilde{M}_{\hat{f}}$ is nonsingular, then Pl-Holant $(f)$ is \#P-hard.

In the course of working with symmetric signatures, we sometimes construct gadgets with signatures that are not symmetric. The power of Lemma 2.28 and Corollary 2.29 is that they apply to such signatures provided the corresponding signature matrix is redundant. Starting from a signature with a non-redundant signature matrix, sometimes one can apply a rotation to obtain a signature with a redundant signature matrix (see Fig. 2).

\section{$3 \mathscr{A}-, \mathscr{P}$-, and $\mathscr{M}$-transformable Signatures}

In this section, we investigate the properties of $\mathscr{A}$-, $\mathscr{P}$-, and $\mathscr{M}$-transformable signatures. Throughout, we define $\alpha=\frac{1+i}{\sqrt{2}}=\sqrt{i}=e^{\frac{\pi i}{4}}$ and use $\mathbf{O}_{2}(\mathbb{C})$ to denote the group of 2 -by-2 orthogonal matrices over $\mathbb{C}$. While the main results in this section assume that the signatures involved are symmetric, we note that some of the lemmas also hold without this assumption.

\subsection{Characterization of $\mathscr{A}$ - and $\mathscr{P}$-transformable Signatures}

$\mathscr{A}$ - and $\mathscr{P}$-transformable signatures have been well studied in previous work [11, 12]. We summarize some useful notions and lemmas here. The three sets $\mathscr{A}_{1}, \mathscr{A}_{2}$, and $\mathscr{A}_{3}$ capture all symmetric $\mathscr{A}$-transformable signatures.

Definition 3.1 A signature $f$ of arity $n$ is in, respectively, $\mathscr{A}_{1}$, or $\mathscr{A}_{2}$, or $\mathscr{A}_{3}$ if it is symmetric and there exist an $H \in \mathbf{O}_{2}(\mathbb{C})$ and a nonzero constant $c \in \mathbb{C}$ 
such that $f$ has the form, respectively, $c H^{\otimes n}\left(\left[\begin{array}{l}1 \\ 1\end{array}\right]^{\otimes n}+\beta\left[\begin{array}{l}1 \\ -1\end{array}\right]^{\otimes n}\right)$, or $c H^{\otimes n}$ $\left(\left[\begin{array}{l}1 \\ i\end{array}\right]^{\otimes n}+\left[\begin{array}{l}1 \\ -i\end{array}\right]^{\otimes n}\right)$, or $c H^{\otimes n}\left(\left[\begin{array}{l}1 \\ \alpha\end{array}\right]^{\otimes n}+i^{r}\left[\begin{array}{l}1 \\ -\alpha\end{array}\right]^{\otimes n}\right)$, where $\beta=\alpha^{t n+2 r}$, $r \in\{0,1,2,3\}$, and $t \in\{0,1\}$.

For $k \in\{1,2,3\}$, when such an orthogonal $H$ exists, we say that $f \in \mathscr{A}_{k}$ with transformation $H$. If $f \in \mathscr{A}_{k}$ with $I_{2}$, then we say $f$ is in the canonical form of $\mathscr{A}_{k}$.

The following lemma characterizes the signatures in $\mathscr{A}_{2}$.

Lemma 3.2 (Lemma 54, p. 1714 in [11]) Let $f$ be a symmetric signature of arity n. Then $f \in \mathscr{A}_{2}$ if and only if $f=c\left(\left[\begin{array}{l}1 \\ i\end{array}\right]^{\otimes n}+\beta\left[\begin{array}{l}1 \\ -i\end{array}\right]^{\otimes n}\right)$ for some nonzero constants $c, \beta \in \mathbb{C}$. Thus signatures in $\mathscr{A}_{2}$ are those with the form $[x, y,-x,-y, \ldots]$ with $y \neq \pm i x$.

Signatures in $\mathscr{A}_{2}$ have the form $Z^{\otimes n}[a, 0, \ldots, 0, b]$ where $[a, 0, \ldots, 0, b]$ is a weighted EQUALITY function with $a b \neq 0$.

Membership in these three sets characterizes the $\mathscr{A}$-transformable signatures.

Lemma 3.3 (Lemma 56, p. 1715 in [11]) Let $f$ be a non-degenerate symmetric signature. Then $f$ is $\mathscr{A}$-transformable if and only if $f \in \mathscr{A}_{1} \cup \mathscr{A}_{2} \cup \mathscr{A}_{3}$.

There is a similar characterization for $\mathscr{P}$-transformable signatures.

Definition 3.4 A signature $f$ of arity $n$ is in $\mathscr{P}_{1}$ if it is symmetric and there exist an $H \in \mathbf{O}_{2}(\mathbb{C})$ and a nonzero $c \in \mathbb{C}$ such that $f=c H^{\otimes n}\left(\left[\begin{array}{l}1 \\ 1\end{array}\right]^{\otimes n}+\beta\left[\begin{array}{l}1 \\ -1\end{array}\right]^{\otimes n}\right)$, where $\beta \neq 0$.

We define $\mathscr{P}_{2}=\mathscr{A}_{2}$. For $k \in\{1,2\}$, when such an $H$ exists, we say that $f \in \mathscr{P}_{k}$ with transformation $H$. If $f \in \mathscr{P}_{k}$ with $I_{2}$, then we say $f$ is in the canonical form of $\mathscr{P}_{k}$.

Lemma 3.5 (Lemma 59, p. 1717 in [11]) Let $f$ be a non-degenerate symmetric signature. Then $f$ is $\mathscr{P}$-transformable if and only if $f \in \mathscr{P}_{1} \cup \mathscr{P}_{2}$.

These classes $\mathscr{A}_{i}$ and $\mathscr{P}_{j}$ contain only symmetric functions. These are transformed classes based on (symmetric functions in) $\mathscr{A}$ and $\mathscr{P}$. They are not subsets of $\mathscr{A}$ and $\mathscr{P}$ respectively. However, $\mathscr{A}$ and $\mathscr{P}$ do contain nonsymmetric functions. The same remark applies to the classes $\mathscr{M}_{k}$ for $k=1,2,3,4$ from Definition 3.9 in Section 3.2 , in relation to $\mathscr{M}$. 
We can define

$$
\operatorname{Stab}(\mathscr{A})=\left\{T \in \mathbf{G L}_{2}(\mathbb{C}) \mid T \mathscr{A} \subseteq \mathscr{A}\right\},
$$

as the stabilizer group of $\mathscr{A}$. It can be verified that $\left[\begin{array}{ll}1 & 0 \\ 0 & i\end{array}\right],\left[\begin{array}{ll}0 & 1 \\ 1 & 0\end{array}\right],\left[\begin{array}{ll}1 & 1 \\ 1 & -1\end{array}\right] \in$ $\operatorname{Stab}(\mathscr{A})$.

\subsection{Characterization of $\mathscr{M}$-transformable Signatures}

Now we develop a similar theory for the $\mathscr{M}$-transformable signatures. Recall from Definition 2.4 that for a signature set $\mathcal{F}$ to be $\mathscr{M}$-transformable, it must be that there exists a $T \in \mathbf{G L}_{2}(\mathbb{C})$ such that $[1,0,1] T^{\otimes 2} \in \mathscr{M}$. Since $[1,0,1]$ is symmetric, $[1,0,1] T^{\otimes 2}$ is also symmetric. However, it is unnecessary to consider all binary signatures in $\mathscr{M}$. We can normalize via right multiplication by elements in

$$
\operatorname{Stab}(\mathscr{M})=\left\{T \in \mathbf{G L}_{2}(\mathbb{C}) \mid T \mathscr{M} \subseteq \mathscr{M}\right\},
$$

the stabilizer group of $\mathscr{M}$. Technically this set is the left stabilizer group of $\mathscr{M}$, but it is easy to see that the left and right stabilizer groups of $\mathscr{M}$ coincide. It is easy to prove that

Proposition 3.6 $\mathrm{Stab}(\mathscr{M})$ is generated by nonzero scalar multiples of $\left[\begin{array}{ll}1 & 0 \\ 0 & v\end{array}\right]$ for any nonzero $v \in \mathbb{C}$ and $X=\left[\begin{array}{ll}0 & 1 \\ 1 & 0\end{array}\right]$.

After this normalization, it is enough to consider cases 1 and 3 in the following proposition.

Proposition 3.7 (Proposition 47, p. 1711 in [11]) Let $T \in \mathbb{C}^{2 \times 2}$ be a matrix. Then the following hold:

1. $[1,0,1] T^{\otimes 2}=[1,0,1]$ if and only if $T \in \mathbf{O}_{2}(\mathbb{C})$;

2. $[1,0,1] T^{\otimes 2}=[1,0, i]$ if and only if there exists an $H \in \mathbf{O}_{2}(\mathbb{C})$ such that $T=H\left[\begin{array}{ll}1 & 0 \\ 0 & \alpha\end{array}\right]$;

3. $[1,0,1] T^{\otimes 2}=[0,1,0]$ if and only if there exists an $H \in \mathbf{O}_{2}(\mathbb{C})$ such that

$$
T=\frac{1}{\sqrt{2}} H\left[\begin{array}{ll}
1 & 1 \\
i & -i
\end{array}\right] \text {. }
$$

Lemma 3.8 Let $\mathcal{F}$ be a set of signatures. Then $\mathcal{F}$ is $\mathscr{M}$-transformable if and only if $\mathcal{F} \subseteq\left[\begin{array}{ll}1 & 1 \\ i & -i\end{array}\right] \mathscr{M}$ or there exists an $H \in \mathbf{S O}_{2}(\mathbb{C})$ such that $\mathcal{F} \subseteq H \mathscr{M}$.

Proof Sufficiency is easily verified by checking that $=2$ is transformed into $\mathscr{M}$ in both cases. In particular, $H$ leaves $=2$ unchanged.

If $\mathcal{F}$ is $\mathscr{M}$-transformable, then by definition, there exists a matrix $T$ such that $(=2) T^{\otimes 2} \in \mathscr{M}$ and $\mathcal{F} \subseteq T \mathscr{M}$. The non-degenerate binary signatures in $\mathscr{M}$ are 
either $[0,1,0]$ or of the form $[1,0, v]$, for $v \neq 0$, up to a scalar. However, notice that $[1,0,1]=[1,0, v]\left[\begin{array}{ll}1 & 0 \\ 0 & v^{-\frac{1}{2}}\end{array}\right]^{\otimes 2}$ and $\left[\begin{array}{ll}1 & 0 \\ 0 & v^{-\frac{1}{2}}\end{array}\right] \in \operatorname{Stab}(\mathscr{M})$. Thus, we only need to consider $[1,0,1]$ and $[0,1,0]$. Now we apply Proposition 3.7.

1. If $(=2) T^{\otimes 2}=[1,0,1]$, then by case 1 of Proposition 3.7, we have $T \in \mathbf{O}_{2}$ (C) . If $T \in \mathbf{S O}_{2}(\mathbb{C})$, then we are done with $H=T$. Otherwise, $T \in \mathbf{O}_{2}(\mathbb{C})-\mathbf{S O}_{2}(\mathbb{C})$. We want to find an $H \in \mathbf{S O}_{2}(\mathbb{C})$ such that $\mathcal{F} \subseteq H \mathscr{M}$. Let $H=T\left[\begin{array}{ll}1 & 0 \\ 0 & -1\end{array}\right] \in$ $\mathbf{S O}_{2}(\mathbb{C})$. Then

$$
\mathcal{F} \subseteq T \mathscr{M}=T\left[\begin{array}{cc}
1 & 0 \\
0 & -1
\end{array}\right] \mathscr{M}=H \mathscr{M}
$$

since $\left[\begin{array}{ll}1 & 0 \\ 0 & -1\end{array}\right] \in \operatorname{Stab}(\mathscr{M})$.

2. If $(=2) T^{\otimes 2}=[0,1,0]$, then by case 3 of Proposition 3.7, there exists an $H \in \mathbf{O}_{2}(\mathbb{C})$ such that $T=\frac{1}{\sqrt{2}} H\left[\begin{array}{ll}1 & 1 \\ i & -i\end{array}\right]$. Therefore $\mathcal{F} \subseteq H\left[\begin{array}{ll}1 & 1 \\ i & -i\end{array}\right] \mathscr{M}$. Furthermore, if $H=\left[\begin{array}{ll}a & b \\ -b & a\end{array}\right] \in \mathbf{S O}_{2}(\mathbb{C})$, then $a^{2}+b^{2}=1$ and

$$
\mathcal{F} \subseteq H\left[\begin{array}{cc}
1 & 1 \\
i & -i
\end{array}\right] \mathscr{M}=\left[\begin{array}{cc}
1 & 1 \\
i & -i
\end{array}\right]\left[\begin{array}{cc}
a+b i & 0 \\
0 & a-b i
\end{array}\right] \mathscr{M}=\left[\begin{array}{cc}
1 & 1 \\
i & -i
\end{array}\right] \mathscr{M}
$$

since $H\left[\begin{array}{ll}1 & 1 \\ i & -i\end{array}\right]=\left[\begin{array}{ll}1 & 1 \\ i & -i\end{array}\right]\left[\begin{array}{ll}a+b i & 0 \\ 0 & a-b i\end{array}\right]$ and $\left[\begin{array}{ll}a+b i & 0 \\ 0 & a-b i\end{array}\right] \in$ $\operatorname{Stab}(\mathscr{M})$. Otherwise, $H=\left[\begin{array}{ll}a & b \\ b & -a\end{array}\right] \in \mathbf{O}_{2}(\mathbb{C})-\mathbf{S O}_{2}(\mathbb{C})$, so $a^{2}+b^{2}=1$ and

$$
\mathcal{F} \subseteq H\left[\begin{array}{cc}
1 & 1 \\
i & -i
\end{array}\right] \mathscr{M}=\left[\begin{array}{cc}
1 & 1 \\
i & -i
\end{array}\right]\left[\begin{array}{cc}
0 & a-b i \\
a+b i & 0
\end{array}\right] \mathscr{M}=\left[\begin{array}{cc}
1 & 1 \\
i & -i
\end{array}\right] \mathscr{M}
$$

since $H\left[\begin{array}{ll}1 & 1 \\ i & -i\end{array}\right]=\left[\begin{array}{ll}1 & 1 \\ i & -i\end{array}\right]\left[\begin{array}{ll}0 & a-b i \\ a+b i & 0\end{array}\right]$ and $\left[\begin{array}{ll}0 & a-b i \\ a+b i & 0\end{array}\right] \in$ $\operatorname{Stab}(\mathscr{M})$.

We use four sets to characterize the $\mathscr{M}$-transformable signatures. The function $\operatorname{Sym}_{n}^{t}(-;-)$ is defined in Definition 2.9.

Definition 3.9 A signature $f$ of arity $n$ is in $\mathscr{M}_{k}$ for $k=1,2,3,4$, if it is symmetric and there exist an $H \in \mathbf{O}_{2}(\mathbb{C})$ and nonzero constants $c, \gamma \in \mathbb{C}$ such that $f$ has the form $(k)$ as follows:

(1) : $c H^{\otimes n}\left(\left[\begin{array}{l}1 \\ 1\end{array}\right]^{\otimes n} \pm i^{n}\left[\begin{array}{l}1 \\ -1\end{array}\right]^{\otimes n}\right) ;$
(2) : $c H^{\otimes n}\left(\left[\begin{array}{l}1 \\ \gamma\end{array}\right]^{\otimes n} \pm\left[\begin{array}{l}1 \\ -\gamma\end{array}\right]^{\otimes n}\right)$ for some $\gamma \neq 0 ;$ 
(3) : $\quad c H^{\otimes n} \operatorname{Sym}_{n}^{n-1}\left(\left[\begin{array}{l}1 \\ 0\end{array}\right] ;\left[\begin{array}{l}0 \\ 1\end{array}\right]\right)$;

(4) : $c H^{\otimes n} \operatorname{Sym}_{n}^{n-1}\left(\left[\begin{array}{l}1 \\ i\end{array}\right] ;\left[\begin{array}{l}1 \\ -i\end{array}\right]\right)$.

Form (3) is $c H^{\otimes n}[0,1,0, \ldots, 0]$, and form (4) is $c(H Z)^{\otimes n}[0,1,0, \ldots, 0]$, where $[0,1,0, \ldots, 0]$ is the EXACT-ONE function of arity $n$.

For $k \in\{1,2,3,4\}$, when such an $H$ exists, we say that $f \in \mathscr{M}_{k}$ with transformation $H$. If $f \in \mathscr{M}_{k}$ with $I_{2}$, then we say $f$ is in the canonical form of $\mathscr{M}_{k}$.

Notice that $\left\{\left[\begin{array}{l}1 \\ i\end{array}\right],\left[\begin{array}{l}1 \\ -i\end{array}\right]\right\}$ is set-wise invariant under any transformation in $\mathbf{O}_{2}(\mathbb{C})$ up to nonzero constants. Using this fact, the following lemma gives a characterization of $\mathscr{M}_{4}$. It says that any signature in $\mathscr{M}_{4}$ is essentially in canonical form.

Lemma 3.10 Let $f$ be a symmetric signature of arity $n$. Then $f \in \mathscr{M}_{4}$ if and only if $f=c \operatorname{Sym}_{n}^{n-1}\left(\left[\begin{array}{l}1 \\ i\end{array}\right] ;\left[\begin{array}{l}1 \\ -i\end{array}\right]\right)$ or $f=c \operatorname{Sym}_{n}^{n-1}\left(\left[\begin{array}{l}1 \\ -i\end{array}\right] ;\left[\begin{array}{l}1 \\ i\end{array}\right]\right)$ for some nonzero constant $c \in \mathbb{C}$.

Proof Suppose $f \in \mathscr{M}_{4}$, so that $f=c H^{\otimes n} \operatorname{Sym}_{n}^{n-1}\left(\left[\begin{array}{l}1 \\ i\end{array}\right] ;\left[\begin{array}{l}1 \\ -i\end{array}\right]\right)$. If $H \in$ $\mathbf{S O}_{2}(\mathbb{C})$, then $H=\left[\begin{array}{ll}a & b \\ -b & a\end{array}\right]$ for some $a, b \in \mathbb{C}$ such that $a^{2}+b^{2}=1$. Since $H\left[\begin{array}{l}1 \\ i\end{array}\right]=(a+b i)\left[\begin{array}{l}1 \\ i\end{array}\right]$ and $H\left[\begin{array}{l}1 \\ -i\end{array}\right]=(a-b i)\left[\begin{array}{l}1 \\ -i\end{array}\right]$, it follows that $f=$ $c(a+b i)^{n-1}(a-b i) \operatorname{Sym}_{n}^{n-1}\left(\left[\begin{array}{l}1 \\ i\end{array}\right] ;\left[\begin{array}{l}1 \\ -i\end{array}\right]\right)$. Otherwise, $H \in \mathbf{O}_{2}(\mathbb{C})-\mathbf{S O}_{2}(\mathbb{C})$, so $H=\left[\begin{array}{ll}a & b \\ b & -a\end{array}\right]$ for some $a, b \in \mathbb{C}$ such that $a^{2}+b^{2}=1$. Then $f=$ $c(a+b i)(a-b i)^{n-1} \operatorname{Sym}_{n}^{n-1}\left(\left[\begin{array}{l}1 \\ -i\end{array}\right] ;\left[\begin{array}{l}1 \\ i\end{array}\right]\right)$.

Now suppose $f=c \operatorname{Sym}_{n}^{n-1}\left(\left[\begin{array}{l}1 \\ i\end{array}\right] ;\left[\begin{array}{l}1 \\ -i\end{array}\right]\right)$ or $f=c \operatorname{Sym}_{n}^{n-1}\left(\left[\begin{array}{l}1 \\ -i\end{array}\right] ;\left[\begin{array}{l}1 \\ i\end{array}\right]\right)$. The first case is already in the standard form of $\mathscr{M}_{4}$. In the second case, we pick $H=\left[\begin{array}{ll}1 & 0 \\ 0 & -1\end{array}\right] \in \mathbf{O}_{2}(\mathbb{C})$. Then $H^{\otimes n} f$ is in the standard form of $\mathscr{M}_{4}$.

We further split $\mathscr{M}_{4}$ into $\mathscr{M}_{4}^{ \pm}$for future use. Define $\mathscr{M}_{4}^{ \pm}=\{f \mid f=$ $\left.c \operatorname{Sym}_{n}^{n-1}\left(\left[\begin{array}{l}1 \\ \pm i\end{array}\right] ;\left[\begin{array}{l}1 \\ \mp i\end{array}\right]\right)\right\}$. In other words, $\mathscr{M}_{4}^{+}$contains signatures of the form $Z^{\otimes n}[0,1,0, \ldots, 0]$ and $\mathscr{M}_{4}^{-}$contains signatures of the form $Z^{\otimes n}[0, \ldots, 0,1,0]$ up to a scalar, where $Z=\left[\begin{array}{ll}1 & 1 \\ i & -i\end{array}\right]$. We will denote $[0,1,0, \ldots, 0]$ of arity $k$

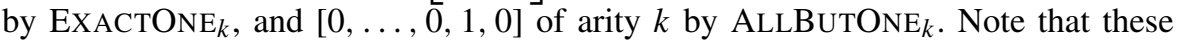
are precisely the PERFECT MATCHING signatures and corresponding reversals. In 
general, the reversal of a symmetric signature $\left[f_{0}, f_{1}, \ldots, f_{n}\right]$ is $\left[f_{n}, \ldots, f_{1}, f_{0}\right]$, corresponding to flipping all input bits 0 and 1. By Definition 2.13 and Lemma 2.19, all signatures in $\mathscr{M}_{4}^{ \pm}$of arity at least 3 belong to $\mathscr{V}^{ \pm}$, respectively.

We note that $\mathscr{M}_{1} \subset \mathscr{A}_{1} \subset \mathscr{P}_{1}$ and $\mathscr{A}_{2}=\mathscr{P}_{2} \subset \mathscr{M}_{2}$. Also note that $\mathscr{P}_{1} \cap \mathscr{M}_{2} \subseteq$ $\mathscr{A}_{1}$. See Fig. 3 for a visual description of the relationships among these sets.

Next we show that $\mathscr{M}_{k}$ for $k=1,2,3,4$ captures all $\mathscr{M}$-transformable signatures.

Lemma 3.11 Let $f$ be a non-degenerate symmetric signature. Then $f$ is $\mathscr{M}$ transformable if and only if $f \in \mathscr{M}_{1} \cup \mathscr{M}_{2} \cup \mathscr{M}_{3} \cup \mathscr{M}_{4}$.

Proof Assume that $f$ is $\mathscr{M}$-transformable of arity $n$. By applying Lemma 3.8 to $\{f\}$, we have $f \in\left[\begin{array}{ll}1 & 1 \\ i & -i\end{array}\right] \mathscr{M}$ or there exists an $H \in \mathbf{S O}_{2}(\mathbb{C})$ such that $f \in H \mathscr{M}$. Proposition 2.10 lists the symmetric signatures in $\mathscr{M}$. Since we are only interested in non-degenerate signatures, we only consider $a, b$, and $\lambda$ that are nonzero. Now we consider the possible cases.

1. Suppose $f \in\left[\begin{array}{ll}1 & 1 \\ i & -i\end{array}\right] \mathscr{M}$.

- If $f=\left[\begin{array}{ll}1 & 1 \\ i & -i\end{array}\right]^{\otimes n}\left(\left[\begin{array}{l}a \\ b\end{array}\right]^{\otimes n} \pm\left[\begin{array}{l}a \\ -b\end{array}\right]^{\otimes n}\right)$ for some nonzero $a, b \in \mathbb{C}$. Let $T=\frac{1-i}{2}\left[\begin{array}{ll}u & v \\ v & -u\end{array}\right]$, where $u=a+b i$ and $v=i(a-b i)$. Then $f=$ $T^{\otimes n}\left(\left[\begin{array}{l}1 \\ 1\end{array}\right]^{\otimes n} \pm i^{n}\left[\begin{array}{l}1 \\ -1\end{array}\right]^{\otimes n}\right)$. Since $T \in \mathbf{O}_{2}(\mathbb{C})$ up to a nonzero factor of $\sqrt{2 a b}$, we have $f \in \mathscr{M}_{1}$.

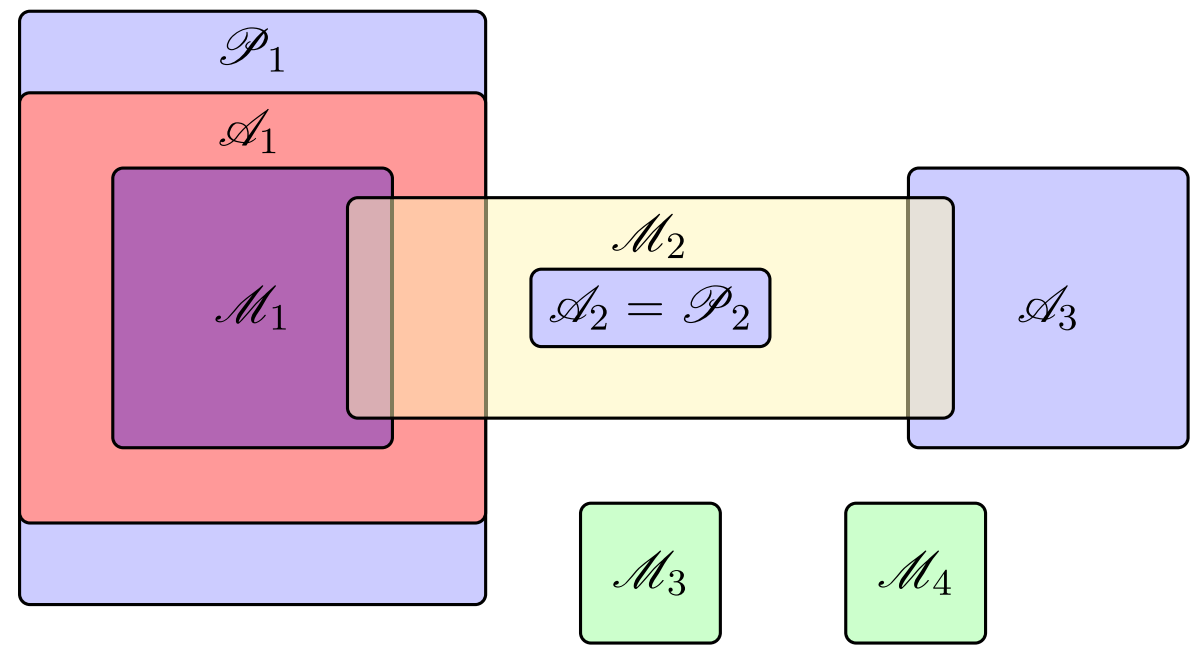

Fig. 3 Relationships among $\mathscr{A}_{1}, \mathscr{A}_{2}, \mathscr{A}_{3}, \mathscr{P}_{1}, \mathscr{P}_{2}, \mathscr{M}_{1}, \mathscr{M}_{2}, \mathscr{M}_{3}$, and $\mathscr{M}_{4}$ 
- Otherwise, if $f=\lambda\left[\begin{array}{ll}1 & 1 \\ i & -i\end{array}\right]^{\otimes n} \operatorname{Sym}_{n}^{n-1}\left(\left[\begin{array}{l}1 \\ 0\end{array}\right] ;\left[\begin{array}{l}0 \\ 1\end{array}\right]\right)$ for some nonzero $\lambda \in$ $\mathbb{C}$. Then we have $f=\lambda \operatorname{Sym}_{n}^{n-1}\left(\left[\begin{array}{l}1 \\ i\end{array}\right] ;\left[\begin{array}{l}1 \\ -i\end{array}\right]\right)$, so $f \in \mathscr{M}_{4}$ by Lemma 3.10 . - Otherwise, $f=\lambda\left[\begin{array}{ll}1 & 1 \\ i & -i\end{array}\right]^{\otimes n} \operatorname{Sym}_{n}^{n-1}\left(\left[\begin{array}{l}0 \\ 1\end{array}\right] ;\left[\begin{array}{l}1 \\ 0\end{array}\right]\right)$ for some nonzero $\lambda \in$ $\mathbb{C}$. Then we have $f=\lambda \operatorname{Sym}_{n}^{n-1}\left(\left[\begin{array}{l}1 \\ -i\end{array}\right] ;\left[\begin{array}{l}1 \\ i\end{array}\right]\right)$, so $f \in \mathscr{M}_{4}$ by Lemma 3.10.

2. Suppose $f \in H \mathscr{M}$.

- If $f=H^{\otimes n}\left(\left[\begin{array}{l}a \\ b\end{array}\right]^{\otimes n} \pm\left[\begin{array}{l}a \\ -b\end{array}\right]^{\otimes n}\right)$ for some nonzero $a, b \in \mathbb{C}$. Then we have the form $f=a^{n} H^{\otimes n}\left(\left[\begin{array}{l}1 \\ \gamma\end{array}\right]^{\otimes n} \pm\left[\begin{array}{l}1 \\ -\gamma\end{array}\right]^{\otimes n}\right)$, where $\gamma=\frac{b}{a}$, so $f \in$ $\mathscr{M}_{2}$.

- Otherwise, if $f=\lambda H^{\otimes n} \operatorname{Sym}_{n}^{n-1}\left(\left[\begin{array}{l}1 \\ 0\end{array}\right] ;\left[\begin{array}{l}0 \\ 1\end{array}\right]\right)$ for some nonzero $\lambda \in \mathbb{C}$. Then $f \in \mathscr{M}_{3}$.

- Otherwise, $f=\lambda H^{\otimes n} \operatorname{Sym}_{n}^{n-1}\left(\left[\begin{array}{l}0 \\ 1\end{array}\right] ;\left[\begin{array}{l}1 \\ 0\end{array}\right]\right)$ for some nonzero $\lambda \in \mathbb{C}$. Let $H^{\prime}=H\left[\begin{array}{ll}0 & 1 \\ 1 & 0\end{array}\right] \in \mathbf{O}_{2}(\mathbb{C})$. Then we have $f=\lambda H^{\prime \otimes n} \operatorname{Sym}_{n}^{n-1}\left(\left[\begin{array}{l}1 \\ 0\end{array}\right] ;\left[\begin{array}{l}0 \\ 1\end{array}\right]\right)$, so $f \in \mathscr{M}_{3}$.

Conversely, if there exists a matrix $H \in \mathbf{O}_{2}(\mathbb{C})$ such that $H^{\otimes n} f$ is in one of the canonical forms of $\mathscr{M}_{1}, \mathscr{M}_{2}, \mathscr{M}_{3}$, or $\mathscr{M}_{4}$, then one can directly check that $f$ is $\mathscr{M}_{\text {- }}$ transformable by Definition 2.4. In fact, the transformations that we applied above are all invertible.

Furthermore, we show that a nontrivial signature $f$ in the set $\mathscr{M}_{3}$ is not $\mathscr{A}$ - or $\mathscr{P}$ transformable. Moreover, the only transformation to make $f$ in $\mathscr{M}$ is very restricted. This is for future use.

Lemma 3.12 Let $f \in \mathscr{M}_{3}$ be a non-degenerate signature of arity $n \geq 3$ with $H \in$ $\mathbf{O}_{2}(\mathbb{C})$. Then $f$ is not $\mathscr{A}$-or $\mathscr{P}$-transformable. Moreover, $f$ is $\mathscr{M}$-transformable with only $H D$ or $H\left[\begin{array}{ll}0 & 1 \\ 1 & 0\end{array}\right]$ D for some diagonal matrix $D$.

Proof Suppose $f=\left[f_{0}, f_{1}, \ldots, f_{n}\right]$. It can be directly verified that symmetric signatures in $\mathscr{A}$ and $\mathscr{P}$ satisfy second order recurrence relations (see Lemma 48, p. 1712 [11]) with distinct eigenvalues, and this property still holds under a holographic transformation. Indeed if $f$ is $\mathscr{A}$ - or $\mathscr{P}$-transformable, then $f$ satisfies a second order recurrence relation $a f_{i}+b f_{i+1}+c f_{i+2}=0$, for $a, b, c \in \mathbb{C}$ such that not all $a, b, c$ are 0 and $b^{2}-4 a c \neq 0$. In other words, the second order recurrence relation has to have distinct eigenvalues. Moreover, this property is preserved by holographic 
transformations [12]. However, $f$ is in $\mathscr{M}_{3}$. Hence $f=H^{\otimes n} \operatorname{EXACTONE}_{n}$ for some $H \in \mathbf{O}_{2}(\mathbb{C})$ up to a nonzero factor. On the other hand, EXACTONE ${ }_{n}$ does not satisfy a second order recurrence with distinct eigenvalues if $n \geq 3$, a contradiction.

Moreover, notice that the only signatures in $\mathscr{M}$ that do not satisfy such second

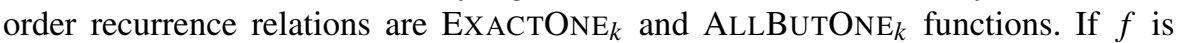
$\mathscr{M}$-transformable, then there exists a transformation $T$ such that $f=T^{\otimes n} g$ for some $g \in \mathscr{M}$ and $[1,0,1] T^{\otimes 2} \in \mathscr{M}$. Hence $g=$ ExACTONE $_{n}$ or AllButOnE . On the other hand $f=H^{\otimes n} \operatorname{EXACTONE}_{n}$ up to a nonzero factor. Therefore

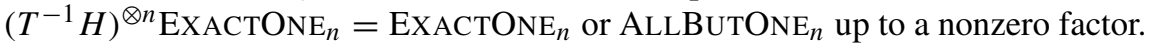

Let $J=T^{-1} H=\left[\begin{array}{ll}x & y \\ z & w\end{array}\right]$ and let $h=J^{\otimes n} \operatorname{ExACTONE}_{n}$. As ExACTONE $\operatorname{Ex}_{n}=$ $\operatorname{Sym}_{n}^{n-1}\left(\left[\begin{array}{l}1 \\ 0\end{array}\right] ;\left[\begin{array}{l}0 \\ 1\end{array}\right]\right), h=\left(\left[\begin{array}{ll}x & y \\ z & w\end{array}\right]\right)^{\otimes n} \operatorname{ExACTONE}_{n}=\operatorname{Sym}_{n}^{n-1}\left(\left[\begin{array}{l}x \\ z\end{array}\right] ;\left[\begin{array}{l}y \\ w\end{array}\right]\right)$. The first and last entries of $h$ are $x^{n-1} y$ and $z^{n-1} w$. As $h=$ EXACTONE $_{n}$ or AlLButONE $_{n}$, we have that $x^{n-1} y=z^{n-1} w=0$. It is easy to see that $x$ and $z$, or $y$ and $w$ cannot be both 0 . Then $x=w=0$ or $y=z=0$. This implies that $J=D$ or $J=D\left[\begin{array}{ll}0 & 1 \\ 1 & 0\end{array}\right]$ for some diagonal matrix $D$. Thus $T=H J^{-1}=H D^{-1}$ or $H\left[\begin{array}{ll}0 & 1 \\ 1 & 0\end{array}\right] D^{-1}$.

Let $g=[x, y, 0, \ldots, 0, z]$ have arity $n \geq 3$, where $x y z \neq 0$. As an example of the theory developed in this section, we discuss the signature $Z^{\otimes n} g$ in the following lemma, which will be used in Lemma 6.6 in the proof of the single signature dichotomy Theorem 6.1.

Lemma 3.13 Let $n \geq 3, g=[x, y, 0, \ldots, 0, z]$ have arity $n$ and $x y z \neq 0$. Then the signature $Z^{\otimes n} g$ is neither $\mathscr{A}$-, $\mathscr{P}$-, $\mathscr{M}$-transformable, nor vanishing.

Remark 1 By Theorem 2.22, for arity $n=3$ or 4 , Lemma 3.13 says that Pl-Holant $\left(Z^{\otimes n} g\right)$ is \#P-hard. After we have proved Theorem 6.1, this lemma will imply that Pl-Holant $\left(Z^{\otimes n} g\right)$ is \#P-hard for all $n \geq 3$.

Proof That $Z^{\otimes n} g$ is not vanishing follows from Lemma 2.19 combined with Corollary 2.18 and Theorem 2.15 . To show that $Z^{\otimes n} g$ is not $\mathscr{A}$-, $\mathscr{P}$-, $\mathscr{M}$-transformable, we only need to show that $Z^{\otimes n} g \notin \mathscr{P}_{1} \cup \mathscr{M}_{2} \cup \mathscr{A}_{3} \cup \mathscr{M}_{3} \cup \mathscr{M}_{4}$ by Lemma 3.3, 3.5 and 3.11, and the fact that $\mathscr{M}_{1} \subset \mathscr{A}_{1} \subset \mathscr{P}_{1}$ and $\mathscr{A}_{2}=\mathscr{P}_{2} \subset \mathscr{M}_{2}$. See Fig. 3.

We first show that $Z^{\otimes n} g \notin \mathscr{P}_{1} \cup \mathscr{M}_{2} \cup \mathscr{A}_{3}$. We say a signature $f=$ $\left[f_{0}, f_{1}, \ldots, f_{n}\right]$ satisfies a second order recurrence of type $\langle a, b, c\rangle$ if $a f_{k}-b f_{k+1}+$ $c f_{k+2}=0$ for $1 \leq k \leq n-2$, for some $a, b$ and $c$ not all zero. Symmetric signatures in $\mathscr{A}, \mathscr{P}, \mathscr{M}$ all satisfy second order recurrences, and this property is invariant under a holographic transformation. So signatures in $\mathscr{P}_{1} \cup \mathscr{M}_{2} \cup \mathscr{A}_{3}$ also satisfy second order recurrences. The recurrence $a f_{k}-b f_{k+1}+c f_{k+2}=0$ has characteristic equation $a-b X+c X^{2}=0$. Suppose $Z^{\otimes n} g \in \mathscr{P}_{1} \cup \mathscr{M}_{2} \cup \mathscr{A}_{3}$, then for some $H \in \mathbf{O}_{2}(\mathbb{C})$, 
$f=H^{\otimes n} Z^{\otimes n} g=(H Z)^{\otimes n} g$ has the canonical form given in Definitions 3.4, 3.9 or 3.1. As such, $f$ satisfies a second order recurrence, and so does $\left(Z^{-1}\right)^{\otimes n} f$. We have $H Z=Z D$ or $Z D\left[\begin{array}{ll}0 & 1 \\ 1 & 0\end{array}\right]$ for some non-singular diagonal $D$. Then $f=Z^{\otimes n} g^{\prime}$, where $g^{\prime}=D^{\otimes n} g$ or $\left(D\left[\begin{array}{ll}0 & 1 \\ 1 & 0\end{array}\right]\right)^{\otimes n} g$. So $g^{\prime}$ has the form $\left[x^{\prime}, y^{\prime}, 0, \ldots, 0, z^{\prime}\right]$ or $\left[x^{\prime}, 0, \ldots, 0, y^{\prime}, z^{\prime}\right]$, with $x^{\prime} y^{\prime} z^{\prime} \neq 0$. We assume the former; the proof is similar for the latter.

It follows that $g^{\prime}=\left(Z^{-1}\right)^{\otimes n} f$ satisfies a second order recurrence. However, for $n \geq 4, g^{\prime}$ does not satisfy any second order recurrence. For a contradiction suppose $g^{\prime}$ does. By $x^{\prime} y^{\prime} z^{\prime} \neq 0, a y^{\prime}-b 0+c 0=0$ gives $a=0, a x^{\prime}-b y^{\prime}+c 0=0$ gives $b=0$, and $a 0-b 0+c z^{\prime}=0$ gives $c=0$; but $a, b, c$ cannot be all zero.

Next suppose $n=3$, and we show that $g^{\prime}=\left(Z^{-1}\right)^{\otimes n} f$ is still impossible. For $\mathscr{P}_{1}$, $f=\left[\begin{array}{l}1 \\ 1\end{array}\right]^{\otimes 3}+\beta\left[\begin{array}{l}1 \\ -1\end{array}\right]^{\otimes 3}$ up to a nonzero constant. It is easy to check that $\left(Z^{-1}\right)^{\otimes n} f$ satisfies a second order recurrence whose two eigenvalues sum to zero. However $g^{\prime}=\left[x^{\prime}, y^{\prime}, 0, z^{\prime}\right]$ has type $\left\langle y^{\prime} z^{\prime}, x^{\prime} z^{\prime},-y^{\prime 2}\right\rangle$, so the sum of its two eigenvalues is $-x^{\prime} z^{\prime} / y^{\prime 2} \neq 0$.

For $\mathscr{M}_{2}, f=\left[\begin{array}{l}1 \\ \gamma\end{array}\right]^{\otimes 3} \pm\left[\begin{array}{l}1 \\ -\gamma\end{array}\right]^{\otimes 3}$. In $\left(Z^{-1}\right)^{\otimes n} f, Z^{-1}\left[\begin{array}{ll}1 & 1 \\ \gamma & -\gamma\end{array}\right]$ has the form $\left[\begin{array}{ll}u & v \\ v & u\end{array}\right]$, and $\left(Z^{-1}\right)^{\otimes n} f=\left[\begin{array}{l}u \\ v\end{array}\right]^{\otimes 3} \pm\left[\begin{array}{l}v \\ u\end{array}\right]^{\otimes 3}$. Thus the weight 1 and weight 2 entries of $\left(Z^{-1}\right)^{\otimes n} f$ are either equal or sum to zero. If $g^{\prime}=\left(Z^{-1}\right)^{\otimes n} f$ this would imply $y^{\prime}=0$, a contradiction.

For $\mathscr{A}_{3}, f=\left[\begin{array}{l}1 \\ \alpha\end{array}\right]^{\otimes n}+i^{r}\left[\begin{array}{l}1 \\ -\alpha\end{array}\right]^{\otimes n}$. (Recall, as defined at the beginning of Section $3, \alpha=\sqrt{i}$.) $Z^{-1}\left[\begin{array}{ll}1 & 1 \\ \alpha & -\alpha\end{array}\right]=\left[\begin{array}{ll}u & v \\ v & u\end{array}\right]$, with $u=1-\alpha i$ and $v=1+\alpha i$. The weight 2 entry of $\left(Z^{-1}\right)^{\otimes n} f$ is $u v^{2}+i^{r} v u^{2}=(u v)\left(v+i^{r} u\right)$. This is nonzero for all $r$. However for $g^{\prime}=\left[x^{\prime}, y^{\prime}, 0, z^{\prime}\right]$, the weight 2 entry is 0 .

It remains to show that $Z^{\otimes n} g \notin \mathscr{M}_{3} \cup \mathscr{M}_{4}$. If $Z^{\otimes n} g \in \mathscr{M}_{3}$, then $Z^{\otimes n} g=c H^{\otimes n} f$ for some $H \in \mathbf{O}_{2}(\mathbb{C})$ and $f=\operatorname{Sym}_{n}^{n-1}\left(\left[\begin{array}{l}1 \\ 0\end{array}\right] ;\left[\begin{array}{l}0 \\ 1\end{array}\right]\right.$. Again $f=\left((c H)^{-1} Z\right)^{\otimes n} g=$ $Z^{\otimes n} g^{\prime}$ for some $g^{\prime}$ having the same form as $g$ or its reversal. Then $g^{\prime}=\left(Z^{-1}\right)^{\otimes n} f$ is the signature $[n, n-2, \ldots,-(n-2),-n]$, up to a nonzero constant. The weight 1 entry and weight $n-1$ entry have the same absolute value. By the form of $g^{\prime}$ this is a contradiction.

Finally if $Z^{\otimes n} g \in \mathscr{M}_{4}$, then by Lemma 3.10, $Z^{\otimes n} g=c Z^{\otimes n} f$, for some nonzero constant $c \in \mathbb{C}$, and $f=\operatorname{Sym}_{n}^{n-1}\left(\left[\begin{array}{l}1 \\ 0\end{array}\right] ;\left[\begin{array}{l}0 \\ 1\end{array}\right]\right)$ or its reversal $\operatorname{Sym}_{n}^{n-1}\left(\left[\begin{array}{l}0 \\ 1\end{array}\right] ;\left[\begin{array}{l}1 \\ 0\end{array}\right]\right.$ ). In either case, after canceling out $Z$, the weight 0 entry is 0 in the expression but not so in $g$; a contradiction. 


\section{Mixing with Vanishing Signatures}

In this section, we prove some hardness results for vanishing signature sets when augmented by other signatures. We first consider the mixing of vanishing signatures with unary and binary signatures. Over general graphs, these cases are handled by Lemma 44 and Lemma 45 (p. 1708) in [11]. One can check that the hardness in Lemma 44 in [11] holds for planar graphs. We state the planar version of Lemma 44 in [11] and provide a proof for completeness. Specifically, the reduction to obtain the signature $f^{\prime \prime}$ is planar and Pl-Holant $\left(f^{\prime \prime}\right)$ is \#P-hard by Theorem 2.22.

Lemma 4.1 Let $f \in \mathscr{V}^{\sigma}$ be a symmetric signature of arity $n$ with $\mathrm{rd}^{\sigma}(f)=d \geq 2$ where $\sigma \in\{+,-\}$. Suppose $v=u^{\otimes m}$ is a symmetric degenerate signature for some unary signature $u$ and some integer $m \geq 1$. If $u$ is not a multiple of $[1, \sigma i]$, then Pl-Holant $(f, v)$ is \#P-hard.

Proof We consider $\sigma=+$ since the other case is similar. Since $f \in \mathscr{V}^{+}$, we have $n>2 d \geq 4$. Under a holographic transformation by $Z$, we have

$$
\operatorname{Pl-Holant}(f, v) \equiv \mathrm{Pl} \text {-Holant }\left(\neq_{2} \mid \hat{f},[a, b]^{\otimes m}\right),
$$

where $\hat{f}=\left(Z^{-1}\right)^{\otimes n} f$ and $[a, b]^{\otimes m}=\left(Z^{-1}\right)^{\otimes m} v$ with $b \neq 0$ since $u$ is not a multiple of $[1, i]$. Moreover, $\hat{f}=\left[\hat{f}_{0}, \hat{f}_{1}, \ldots, \hat{f}_{d}, 0, \ldots, 0\right]$ with $\hat{f}_{d} \neq 0$ by Lemma 2.19.

We get $\widehat{f}^{\prime}=\left[\hat{f}_{d-2}, \hat{f}_{d-1}, \hat{f}_{d}, 0, \ldots, 0\right]$ of arity $n-2 d+4$ by $d-2$ self-loops via $\neq_{2}$ on $\hat{f}$. This signature is on the right hand side of the bipartite Holant problem Pl-Holant $\left(\neq_{2} \mid \hat{f},[a, b]^{\otimes m}\right)$. With two more self-loops, we get $[1,0]^{\otimes(n-2 d)}$, also on the right.

We claim that we can use $[1,0]^{\otimes(n-2 d)}$ and $[a, b]^{\otimes m}$ to create $[a, b]^{\otimes(n-2 d)}$. Let $t=\operatorname{gcd}(m, n-2 d)$. If $n-2 d>m$, then we connect $[a, b]^{\otimes m}$ to $[1,0]^{\otimes(n-2 d)}$ via $\neq 2$ to get $[1,0]^{\otimes(n-2 d-m)}$ up to a nonzero factor $b^{m}$. We repeat this process until we get a tensor power $[1,0]^{\otimes \ell}$ for some $\ell \leq m$. We can do a similar construction if $m>n-2 d$. Repeat this process, which is a subtractive Euclidean algorithm. Halt upon getting both $[1,0]^{\otimes t}$ and $[a, b]^{\otimes t}$. Then we combine $\frac{n-2 d}{t}$ copies of $[a, b]^{\otimes t}$ to get $[a, b]^{\otimes(n-2 d)}$.

Now connecting $[a, b]^{\otimes(n-2 d)}$ back to $\widehat{f}^{\prime}$ via $\neq 2$, gives $\widehat{f^{\prime \prime}}=$ $\left[\widehat{f}_{0}^{\prime \prime}, \widehat{f}_{1}^{\prime \prime}, \widehat{f}_{2}^{\prime \prime}, 0,0\right]$ of arity 4. Moreover, $\widehat{f}_{2}^{\prime \prime}=b^{n-2 d} \hat{f}_{d} \neq 0$. Notice that

$$
\operatorname{Pl-Holant}\left(\neq_{2} \mid\left[{\widehat{f^{\prime \prime}}}_{0},{\widehat{f^{\prime \prime}}}_{1},{\widehat{f^{\prime \prime}}}_{2}, 0,0\right]\right) \equiv \operatorname{Pl}-\text { Holant }\left(\neq_{2} \mid[0,0,1,0,0]\right) \text {, }
$$

the latter being counting Eulerian Orientations over planar 4-regular graphs, which is \#P-hard by Corollary 2.29 (or more directly by [26, Theorem 3.7]). Thus, $\mathrm{Pl}$-Holant $(f, v)$ is \#P-hard.

Next come binary signatures. The statement of Lemma 45 (p. 1708) in [11] must be modified to rule out a planar tractable case (which is proved \#P-hard for general graphs in Lemma 45 in [11]). Excluding this planar tractable case, there is one more nonplanar reduction in the proof of Lemma 45 in [11]. This reduction is used to show 
that Holant $\left(\neq_{2} \mid\{[t, 1,0,0,0],[c, 0,1]\}\right)$ is \#P-hard when $c \neq 0$ (but the gadget in Figure 12a of [11] (p. 1710) is nonplanar). In the following lemma, we first show that this problem Holant $\left(\neq_{2} \mid\{[t, 1,0,0,0],[c, 0,1]\}\right)$ remains \#P-hard even restricted to planar graphs provided $t \neq 0$. If $t=0$, then all signatures belong to $\mathscr{M}$ and the problem is tractable.

Lemma 4.2 Let $c, t \in \mathbb{C}$. If $c t \neq 0$, then Pl-Holant $\left(\neq_{2} \mid[t, 1,0,0,0],[c, 0,1]\right)$ is \#P-hard.

Proof By connecting two copies of $\neq 2$ to either side of $[c, 0,1]$, we get the signature $[1,0, c]$ on the left. Clearly Pl-Holant $([1,0, c] \mid[t, 1,0,0,0]) \leq_{T}$ Pl-Holant $\left(\neq_{2} \mid[t, 1,0,0,0],[c, 0,1]\right)$. Then under a holographic transformation by $T^{-1}$, where $T=\left[\begin{array}{cc}1 & 0 \\ 0 & \sqrt{c}\end{array}\right]$, we have

$$
\begin{aligned}
& \text { Pl-Holant }([1,0, c] \mid[t, 1,0,0,0]) \equiv \operatorname{Pl-Holant}\left([1,0, c]\left(T^{-1}\right)^{\otimes 2} \mid T^{\otimes 4}[t, 1,0,0,0]\right) \\
& \equiv \text { Pl-Holant }([1,0,1] \mid[t, \sqrt{c}, 0,0,0]) \\
& \equiv \text { Pl-Holant }([t, \sqrt{c}, 0,0,0]) \text {. }
\end{aligned}
$$

The last problem is \#P-hard by Corollary 2.23 after dividing by $\sqrt{c}$.

Next we prove the planar version of Lemma 45 (p. 1708) in [11] using Lemma 4.2. We have to rule out the planar tractable case $f \in \mathscr{M}_{4}^{ \pm}$. Also note that if $f \in \mathscr{V}^{ \pm}$ is a symmetric non-degenerate signature, then $f$ has arity at least 3 . This is because a unary signature is degenerate, and if a binary symmetric signature $f$ is vanishing, then its vanishing degree is greater than 1 , hence at least 2 , and therefore $f$ is also degenerate. In the following lemma, we explicitly state this condition $\operatorname{arity}(f) \geq 3$.

Lemma 4.3 Let $f \in \mathscr{V}^{\sigma}$ be a symmetric non-degenerate signature of arity $n \geq 3$ for some $\sigma \in\{+,-\}$. Suppose $h$ is a non-degenerate binary signature. If $f \notin \mathscr{M}_{4}^{\sigma}$ and $h \notin \mathscr{R}_{2}^{\sigma}$, then Pl-Holant $(f, h)$ is \#P-hard.

Proof We consider $\sigma=+$ since the other case is similar. Under a $Z$ transformation,

$$
\operatorname{Pl-Holant}(f, h) \equiv \mathrm{Pl} \text {-Holant }\left(\neq_{2} \mid \hat{f}, \hat{h}\right),
$$

where $\hat{f}=\left(Z^{-1}\right)^{\otimes n} f$ and $\hat{h}=\left(Z^{-1}\right)^{\otimes 2} h$. Since $h \notin \mathscr{R}_{2}^{+}$, we may assume that $\hat{h}=[a, b, 1]$ by Lemma 2.19 with a nonzero entry $\hat{h}_{2}$, here we normalized to 1 . Moreover since $h$ is non-degenerate, so is $\hat{h}$, and therefore $b^{2} \neq a$.

We prove the lemma by induction on the arity of $f$ (or equivalently $\hat{f}$ ). There are two base cases, $n=3$ and $n=4$. However, the arity 3 case is easily reduced to the arity 4 case. We show this first, and then show that the lemma holds in the arity 4 case.

Assume $n=3$. Since $f \in \mathscr{V}^{+}$, by Definition 2.13 , Corollary 2.18 and Lemma 2.14, we have the form $\hat{f}=\left[\hat{f}_{0}, \hat{f}_{1}, 0,0\right]$. Moreover, since $f$ is nondegenerate, we have $\hat{f}_{1} \neq 0$ and we can normalize it to 1 . So we have the form 
Fig. 4 Circle vertices are assigned $[t, 1,0,0]$ and the square vertex is assigned $\neq 2$

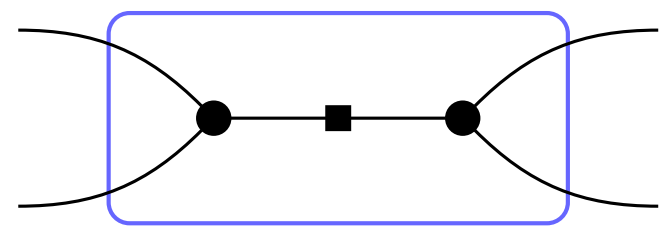

$\hat{f}=[t, 1,0,0]$. By Lemma 2.19, we have $t \neq 0$ as $f \notin \mathscr{M}_{4}^{+}$. Consider the gadget in Fig. 4 . We assign $\hat{f}$ to the circle vertices and $\neq_{2}$ to the square vertex. Let $\hat{f}^{\prime}$ be the signature of the resulting gadget. The signature $\hat{f}^{\prime}$ may not seem symmetric by construction, but in fact it is, and it has signature $\hat{f}^{\prime}=[2 t, 1,0,0,0]$. The crucial observation is that it takes the same value 0 on inputs 1010 and 1100 , where bits are ordered counterclockwise, starting from an arbitrary edge. (Note that $\hat{f}^{\prime}$ outputs value 0 even when both copies of $\hat{f}$ have 01 on the dangling edges.) This finishes our reduction to $n=4$.

Now we consider the base case of $n=4$. Since $f \in \mathscr{V}^{+}$, we have $\mathrm{vd}^{+}(f)>2$ and $\mathrm{rd}^{+}(f)<2$. As $f$ is not degenerate, $\mathrm{rd}^{+}(f) \notin\{-1,0\}$. This implies that $\mathrm{rd}^{+}(f)=1$ and by Lemma $2.19, \hat{f}=[t, 1,0,0,0]$. Moreover, $t \neq 0$ since $f \notin \mathscr{M}_{4}^{+}$.

Our next goal is to show that we can realize a signature of the form $[c, 0,1]$ with $c \neq 0$. Then Pl-Holant $\left(\neq_{2} \mid[t, 1,0,0,0],[c, 0,1]\right) \leq \operatorname{Pl-Holant}(f, h)$. Then it follows from Lemma 4.2 that the problem Pl-Holant $\left(\neq_{2} \mid[t, 1,0,0,0],[c, 0,1]\right)$ is \#P-hard.

Recall that $\hat{h}=[a, b, 1]$ with $b^{2} \neq a$. If $b=0$, then $\hat{h}$ is what we want since in this case $a=a-b^{2} \neq 0$.

Otherwise $b \neq 0$. By connecting $\hat{h}$ to $\hat{f}$ via $\neq 2$, we get $[t+2 b, 1,0]$. If $t \neq-2 b$, then by Lemma 2.20, we can interpolate any binary signature of the form $[v, 1,0]$. Otherwise $t=-2 b$. Then we connect two copies of $\hat{h}$ via $\neq 2$, and get $\widehat{h}^{\prime}=[2 a b, a+$ $\left.b^{2}, 2 b\right]$. By connecting this $\widehat{h}^{\prime}$ to $\hat{f}$ via $\neq 2$, we get $\left[2\left(a-b^{2}\right), 2 b, 0\right]$, using $t=-2 b$. Since $a \neq b^{2}$ and $b \neq 0$, we can once again interpolate any $[v, 1,0]$ by Lemma 2.20.

Hence, we have the signature $[v, 1,0]$, where $v \in \mathbb{C}$ is for us to choose. We construct the gadget in Fig. 5 with the circles assigned $[v, 1,0]$, the squares assigned $\neq_{2}$, and the triangle assigned $[a, b, 1]$. The resulting gadget has signature $[a+2 b v+$ $\left.v^{2}, b+v, 1\right]$, which can be verified by the matrix product

$$
\left[\begin{array}{ll}
v & 1 \\
1 & 0
\end{array}\right]\left[\begin{array}{ll}
0 & 1 \\
1 & 0
\end{array}\right]\left[\begin{array}{ll}
a & b \\
b & 1
\end{array}\right]\left[\begin{array}{ll}
0 & 1 \\
1 & 0
\end{array}\right]\left[\begin{array}{ll}
v & 1 \\
1 & 0
\end{array}\right]=\left[\begin{array}{cc}
a+2 b v+v^{2} & b+v \\
b+v & 1
\end{array}\right] .
$$

By setting $v=-b$, we get $[c, 0,1]$, where $c=a-b^{2} \neq 0$.

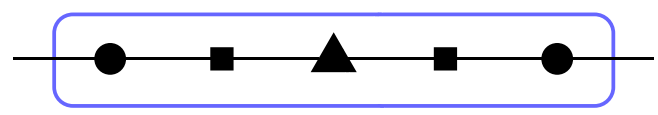

Fig. 5 A sequence of binary gadgets that forms another binary gadget. The circles are assigned $[v, 1,0]$, the squares are assigned $\neq_{2}$, and the triangle is assigned $[a, b, 1]$ 
Now we do the induction step. Assume $n \geq 5$. The construction is essentially identical to the case $n=4$, but for clarity we spell it out. Since $f$ is non-degenerate, $\mathrm{rd}^{+}(f) \geq 1$. If $\mathrm{rd}^{+}(f)=1$, then $\hat{f}=[t, 1,0, \ldots, 0]$ for some $t \neq 0$. We connect $\hat{h}$ to $\hat{f}$ via $\neq 2$, getting $[t+2 b, 1,0, \ldots, 0]$ of arity $n-2 \geq 3$. If $t+2 b \neq 0$, then we are done by induction hypothesis. Otherwise $t=-2 b$, and we connect two $\hat{h}$ together via $\neq_{2}$. The signature is $\hat{h}^{\prime}=\left[2 a b, b^{2}+a, 2 b\right]$. Connect $\hat{h}^{\prime}$ to $\hat{f}$ via $\neq_{2}$. We get $\left[-4 b^{2}+2\left(b^{2}+a\right), 2 b, 0, \ldots, 0\right]=\left[2\left(a-b^{2}\right), 2 b, 0, \ldots, 0\right]$. If $b=0$, then $t=0$. Contradiction. Hence $b \neq 0$, and also $a-b^{2} \neq 0$ since $\hat{h}$ is nondegenerate. Then we can apply induction hypothesis on $\left[2\left(a-b^{2}\right), 2 b, 0, \ldots, 0\right]$.

The case left is that $\mathrm{rd}^{+}(f)=d \geq 2$. Then $\hat{f}=\left[\hat{f}_{0}, \hat{f}_{1}, \ldots, \hat{f}_{d}, 0, \ldots, 0\right]$ with $\hat{f}_{d} \neq 0$ by Lemma 2.19. We form a self-loop of $\hat{f}$ via $\neq_{2}$, getting the signature $\hat{f}^{\prime \prime}=\left[\hat{f}_{1}, \ldots, \hat{f}_{d}, 0, \ldots, 0\right]$ of arity $n-2 \geq 3$. Since $d \geq 2, \hat{f}^{\prime \prime}$ is non-degenerate and $f^{\prime \prime}=Z^{\otimes(n-2)} \hat{f}^{\prime \prime} \in \mathscr{V}^{+}$. If $f^{\prime \prime} \notin \mathscr{M}_{4}^{+}$, then apply the induction hypothesis and we are done. Otherwise, by the form of $\hat{f}^{\prime \prime}$, we have $d=2$ and $\hat{f}_{1}=0$, and so we may assume $\hat{f}=\left[\hat{f}_{0}, 0,1,0, \ldots, 0\right]$ since $\hat{f}_{2} \neq 0$.

In this case, we connect $\hat{h}$ to $\hat{f}$ via $\neq_{2}$, getting $\hat{f}^{\prime \prime \prime}=\left[a+\hat{f}_{0}, 2 b, 1,0, \ldots, 0\right]$ of arity $n-2 \geq 3$. If $n \geq 7$, then we can apply the induction hypothesis. If $n=6$, then $\hat{f}^{\prime \prime \prime}=\left[a+\hat{f}_{0}, 2 b, 1,0,0\right]$ of arity 4 . Notice that Pl-Holant $([0,1,0]$ $\left.\left[a+\hat{f}_{0}, 2 b, 1,0,0\right]\right)$ is equivalent to Pl-Holant $([0,1,0] \mid[0,0,1,0,0])$, which is counting Eulerian orientations in 4-regular planar graphs. Then Pl-Holant $\left(\neq_{2} \mid \hat{f}^{\prime \prime \prime}\right)$ is \#P-hard by Corollary 2.29.

The only case left now is when $n=5$ and $\hat{f}=\left[\hat{f}_{0}, 0,1,0,0,0\right]$. We do two selfloops on $\hat{f}$ via $\neq_{2}$ to get $[1,0]$. Then connect $[1,0]$ to $\hat{h}$ via $\neq_{2}$ and get $[b, 1]$. At last, connect $[b, 1]$ to $\hat{f}$ via $\neq_{2}$, resulting in $\left[\hat{f}_{0}, b, 1,0,0\right]$. Similar to the case above, this is counting Eulerian orientations in 4-regular planar graphs, and is \#P-hard by Corollary 2.29.

If $f \in \mathscr{M}_{4}^{ \pm}$, there is an additional case for binary signatures.

Lemma 4.4 Let $f \in \mathscr{M}_{4}^{\sigma}$ be a symmetric non-degenerate signature with $\sigma \in\{+,-\}$ of arity $k \geq 3$. Suppose $h$ is a non-degenerate binary signature such that $h \notin \mathscr{R}_{2}^{\sigma}$ and $h$ is not a multiple of $Z^{\otimes 2}[a, 0,1]$ for any $a \neq 0$. Then $\mathrm{Pl}-\operatorname{Holant}(f, h)$ is \#P-hard.

Proof We assume $f \in \mathscr{M}_{4}^{+}$since the other case is similar. Suppose $h=Z^{\otimes 2}[a, b, c]$ for some $a, b, c \in \mathbb{C}$. Since $h \notin \mathscr{R}_{2}^{+}$, we have $c \neq 0$, so we assume $c=1$. Moreover $b \neq 0$. This is because, if $b=0$ then either $h$ is degenerate or is a multiple of $Z^{\otimes 2}[a, 0,1]$ for some $a \neq 0$. Either case is a contradiction. Then under a holographic transformation by $Z$, the problem becomes Pl-Holant $\left(\neq_{2} \mid \operatorname{ExACTONE}_{k},[a, b, 1]\right)$. If we connect two copies of EXACTONE $k$ via $\neq_{2}$, we get $\operatorname{EXACTONE}_{2 k-2}$. Hence we may assume that $k \geq 5$. Then we connect $[a, b, 1]$ to $\operatorname{EXACTONE}_{k}$ via $\neq_{2}$, and get $[2 b, 1,0, \ldots, 0]$ of arity $k-2 \geq 3$. Since $b \neq 0$, Pl-Holant $(f, h)$ is \#P-hard by Lemma 4.3.

Next we consider mixing signatures from $\mathscr{V}^{+}$and $\mathscr{V}^{-}$. This is a planar version of Lemma 46 (p. 1711) in [11]. However, for planar graphs, there is a tractable case 
when one signature is in $\mathscr{M}_{4}^{+}$and the other is in $\mathscr{M}_{4}^{-}$. This case was shown to be \#Phard over general graphs by Lemma 43 in [11] (p. 1704) using a nonplanar reduction. One can check that the rest of the proof of Lemma 46 in [11] holds for planar graphs. For completeness we include a proof.

Lemma 4.5 Let $f \in \mathscr{V}^{+}$and $g \in \mathscr{V}^{-}$be symmetric non-degenerate signatures of arities $\geq 3$ respectively. If $f \notin \mathscr{M}_{4}^{+}$or $g \notin \mathscr{M}_{4}^{-}$then $\mathrm{Pl}$-Holant $(f, g)$ is \#P-hard.

Proof Let $\mathrm{rd}^{+}(f)=d, \mathrm{rd}^{-}(g)=d^{\prime}, \operatorname{arity}(f)=n$ and $\operatorname{arity}(g)=n^{\prime}$, then $2 d<n$ and $2 d^{\prime}<n^{\prime}$. Under a holographic transformation by $Z=\left[\begin{array}{ll}1 & 1 \\ i & -i\end{array}\right]$, we have

$$
\text { Pl-Holant }\left(=_{2} \mid f, g\right) \equiv_{T} \text { Pl-Holant }\left(\neq_{2} \mid \hat{f}, \hat{g}\right) \text {, }
$$

where $\hat{f}=\left(Z^{-1}\right)^{\otimes n} f=\left[\hat{f}_{0}, \ldots, \hat{f}_{d}, 0, \ldots, 0\right]$ and $\hat{g}=\left(Z^{-1}\right)^{\otimes n^{\prime}} g=$ $\left[0, \ldots, 0, \hat{g}_{d^{\prime}}, \ldots, \hat{g}_{0}\right]$ due to Lemma 2.19. Moreover $\hat{f}_{d} \neq 0$ and $\hat{g}_{d^{\prime}} \neq 0$.

If $d \geq 2$, we can do $d^{\prime}$ many self-loops of $\neq 2$ on $\hat{g}$, getting $\hat{g}^{\prime}=\left[0, \ldots, 0, \hat{g}_{d^{\prime}}\right]$ of arity $n^{\prime}-2 d^{\prime} \geq 1$. Thus $g^{\prime}$ is $Z^{\otimes\left(n^{\prime}-2 d^{\prime}\right)} \hat{g}^{\prime}=[1,-i]^{\otimes\left(n^{\prime}-2 d^{\prime}\right)}$ up to a nonzero constant. We apply Lemma 4.1 to derive that $\operatorname{Pl-Holant}(f, g)$ is \#P-hard. If $d^{\prime} \geq 2$, we can similarly get $[1, i]^{\otimes(n-2 d)}$ and apply Lemma 4.1. Thus we can assume that $d=d^{\prime}=1$.

So up to nonzero constants, we have $\hat{f}=[a, 1,0, \ldots, 0]$ and $\hat{g}=[0, \ldots, 0,1, b]$ for some $a, b \in \mathbb{C}$. We can assume that $f \notin \mathscr{M}_{4}^{+}$and $a \neq 0$. The case of $b \neq 0$ is similar. We show that it is always possible to get two such signatures of the same arity $\min \left\{n, n^{\prime}\right\}$. Suppose $n>n^{\prime}$. We form a loop from $\hat{f}$ via $\neq_{2}$. It is easy to see that this signature is the degenerate signature $2[1,0]^{\otimes(n-2)}$. Similarly, we can form a loop from $\hat{g}$ and can get $2[0,1]^{\otimes\left(n^{\prime}-2\right)}$. Thus we have both $[1,0]^{\otimes(n-2)}$ and $[0,1]^{\otimes\left(n^{\prime}-2\right)}$. We can connect all $n^{\prime}-2$ edges of the second to the first, connected by $\neq_{2}$. This gives $[1,0]^{\otimes\left(n-n^{\prime}\right)}$. We can continue subtracting the smaller arity from the larger one. We continue this process in a subtractive version of the Euclidean algorithm, and end up with both $[1,0]^{\otimes t}$ and $[0,1]^{\otimes t}$, where $t=\operatorname{gcd}\left(n-2, n^{\prime}-2\right)=\operatorname{gcd}\left(n-n^{\prime}, n^{\prime}-2\right)$. In particular, $t \mid n-n^{\prime}$ and by taking $\frac{n-n^{\prime}}{t}$ copies of $[0,1]^{\otimes t}$, we can get $[0,1]^{\otimes\left(n-n^{\prime}\right)}$. Connecting this back to $\hat{f}$ via $\neq_{2}$, we get a symmetric signature of arity $n^{\prime}$ consisting of the first $n^{\prime}+1$ entries of $\hat{f}$. A similar proof works when $n^{\prime}>n$.

Thus we may assume $n=n^{\prime}$. As shown above we also have $[0,1]^{\otimes(n-2)}$. Connecting $[0,1]^{\otimes(n-2)}$ to $\hat{f}=[a, 1,0, \ldots, 0]$ via $\neq 2$ we get $\hat{h}=[a, 1,0]$. Recall that $a \neq 0$. Translating this back by $Z$, we have a binary signature $h \notin \mathscr{R}_{2}^{-}$and $h$ is not a multiple of $Z^{\otimes 2}[c, 0,1]$ for any $c \neq 0$. Since $g \in \mathscr{V}^{-}$, by Lemma 4.3 or Lemma 4.4, $\mathrm{Pl}-$ Holant $(g, h)$ is \#P-hard. Hence Pl-Holant $(f, g)$ is also \#P-hard.

When signatures in both $\mathscr{M}_{4}^{+}$and $\mathscr{M}_{4}^{-}$appear, we show that the only degenerate signatures that mix must also be vanishing.

Lemma 4.6 Let $f \in \mathscr{M}_{4}^{+}$and $g \in \mathscr{M}_{4}^{-}$be two non-degenerate signatures of arity $\geq 3$. Let $v=u^{\otimes m}$ be a degenerate signature for some unary signature $u$ and some integer $m \geq 1$. If $u$ is not a multiple of $[1, \pm i]$, then Pl-Holant $(f, g, v)$ is \#P-hard. 
Proof Suppose $f$ is of arity $n$ and $g$ of arity $\ell$. Under a holographic transformation by $Z$, we have

$$
\text { Pl-Holant }(f, g, v) \equiv \mathrm{Pl-Holant}\left(\neq_{2} \mid \operatorname{ExACTONE}_{n}, \operatorname{AllButOnE}_{\ell},[a, b]^{\otimes m}\right),
$$

where $a b \neq 0$. Notice that $v$ is transformed to $\left(Z^{-1} u\right)^{\otimes m}=[a, b]^{\otimes m}$. We have $a b \neq 0$ since $u$ is not a multiple of $[1, \pm i]$. First we get $[1,0]^{\otimes(n-2)}$ by a self-loop via

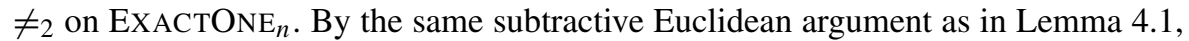
we can realize $[a, b]^{\otimes(n-2)}$ by $[1,0]^{\otimes(n-2)}$ and $[a, b]^{\otimes m}$. Connecting $[a, b]^{\otimes(n-2)}$ to EXACTONE $_{n}$ via $\neq 2$ we get a binary signature $h=\left[(n-2) a b^{n-3}, b^{n-2}, 0\right]$. After transforming back, we have

$$
\text { Pl-Holant }\left(g, Z^{\otimes 2} h\right) \leq_{T} \text { Pl-Holant }(f, g, v) .
$$

However $Z^{\otimes 2} h \notin \mathscr{R}_{2}^{-}$by Lemma 2.19 and it is not a multiple of $Z^{\otimes 2}[c, 0,1]$ for any $c \neq 0$. Hence Pl-Holant $(f, g, v)$ is \#P-hard by Lemma 4.4 , where $\left(g, Z^{\otimes 2} h\right)$ plays the role of " $(f, h)$ " in Lemma 4.4 and $\sigma=-$.

We also consider the mixing of vanishing signatures with those in $\mathscr{P}_{2}$.

Lemma 4.7 Let $f \in \mathscr{V} \backslash \mathscr{M}_{4}$ and $g \in \mathscr{P}_{2}$ be two non-degenerate signatures with arities $m$ and $n$ respectively. If $m, n \geq 3$, then $\mathrm{Pl}-H o l a n t ~(f, g)$ is \#P-hard.

Proof We claim that it suffices to consider $f \in \mathscr{V}^{+} \backslash \mathscr{M}_{4}$ and $g=\left[\begin{array}{l}1 \\ i\end{array}\right]^{\otimes n}+$ $\left[\begin{array}{l}1 \\ -i\end{array}\right]^{\otimes n}$. By Lemma 3.2, we know that $g=\left[\begin{array}{l}1 \\ i\end{array}\right]^{\otimes n}+\beta\left[\begin{array}{l}1 \\ -i\end{array}\right]^{\otimes n}$ for some $\beta \neq 0$ up to a nonzero scalar. Under a holographic transformation by $T=Z\left[\begin{array}{ll}1 & 0 \\ 0 & \beta^{\frac{1}{n}}\end{array}\right] Z^{-1}$, which is orthogonal up to a nonzero factor, we have $\hat{g}=\left(T^{-1}\right)^{\otimes n} g=\left[\begin{array}{l}1 \\ i\end{array}\right]^{\otimes n}+$ $\left[\begin{array}{c}1 \\ -i\end{array}\right]^{\otimes n}$. Now $\mathscr{M}_{4}$ is closed under orthogonal transformations by definition, and $\mathscr{V}$ is closed under orthogonal transformations by Lemma 2.14. Thus, we still have a signature $\hat{f}=\left(T^{-1}\right)^{\otimes n} f$ such that $\hat{f} \in \mathscr{V} \backslash \mathscr{M}_{4}$. If $\hat{f} \in \mathscr{V}^{-}$, then under a holographic transformation by $D=\left[\begin{array}{cc}1 & 0 \\ 0 & -1\end{array}\right]$, we have $\hat{f} \in \mathscr{V}^{+}$. Furthermore, $\hat{g}$ is invariant under $D$. This proves the claim.

Now we assume that $f \in \mathscr{V}^{+} \backslash \mathscr{M}_{4}$ and $g=\left[\begin{array}{l}1 \\ i\end{array}\right]^{\otimes n}+\left[\begin{array}{l}1 \\ -i\end{array}\right]^{\otimes n}$. By Corollary 2.18, we have $\operatorname{rd}^{+}(f)=d<\frac{m}{2}$. Under a holographic transformation by $Z$, we have

$$
\begin{gathered}
\text { Pl-Holant }(=2 \mid f, g) \equiv \text { Pl-Holant }\left([1,0,1] Z^{\otimes 2} \mid Z^{-1}\{f, g\}\right) \\
\equiv \text { Pl-Holant }\left(\neq \neq_{2} \mid \hat{f},=_{n}\right),
\end{gathered}
$$


where $\hat{f}=\left(Z^{-1}\right)^{\otimes m} f$. By Lemma 2.19, the support of $\hat{f}$ is on entries with Hamming weight at most $d$ and includes the entry of Hamming weight exactly $d$. Now $f \notin \mathscr{M}_{4}$, so by Lemma 3.10, we either have $d=1$ and $\hat{f}=\left[\hat{f}_{0}, 1,0, \ldots, 0\right]$ with $\hat{f}_{0} \neq 0$ or $d \geq 2$ and $\hat{f}=\left[\hat{f}_{0}, \hat{f}_{1}, \ldots, \hat{f}_{d-1}, 1,0, \ldots, 0\right]$ (and up to a nonzero scalar in either case).

In the first case, a self-loop on $\hat{f}$ via $\neq 2$ gives $[1,0]^{\otimes(m-2)}$ on the right side. Let $r=\operatorname{gcd}(n, m-2)$, and let $\ell_{1}, \ell_{2}$ be two positive integers such that $\ell_{1} n-\ell_{2}(m-$ $2)=r$. We connect $\ell_{1}$ copies of $={ }_{n}$ with $\ell_{2}$ copies of $[1,0]^{\otimes(m-2)}$ via $\neq 2$ 's to get $[0,1]^{\otimes r}$. Since $r \mid m-2$, we can also realize $[0,1]^{\otimes(m-2)}$ by putting $\frac{m-2}{r}$ copies of $[0,1]^{\otimes r}$ together. Now connect $[0,1]^{\otimes(m-2)}$ to $\hat{f}$ via $\neq_{2}$. The resulting signature is $\left[\hat{f}_{0}, 1,0\right]$. We can also move $=_{n}$ to the left using $n$ copies of $\neq_{2}$. Hence, we have $\operatorname{Pl-Holant}\left(=_{n} \mid\left[\hat{f}_{0}, 1,0\right]\right) \leq_{T}$ Pl-Holant $\left(\neq_{2} \mid \hat{f},=_{n}\right)$. The former problem is \#Phard by Theorem 2.24 since $\hat{f}_{0} \neq 0$, so the latter problem is \#P-hard as well.

In the second case, we have $m \geq 5$ since $2 \leq d<\frac{m}{2}$. Furthermore, we may assume that $d=2$, since otherwise can we do $d-2$ self-loops on $\hat{f}$ via $\neq_{2}$. With this assumption, we do two self-loops on $\hat{f}$ via $\neq_{2}$ to get $[1,0]^{\otimes(m-4)}$ on the right side. By a similar argument as in the previous case, we can construct $[0,1]^{\otimes(m-4)}$ by using $[1,0]^{\otimes(m-4)}$ and $={ }_{n}$ via $\neq 2$. Now connect $[0,1]^{\otimes(m-4)}$ back to $\hat{f}$ via $\neq 2$. We get the arity 4 signature $\left[\hat{f}_{0}, \hat{f}_{1}, 1,0,0\right]$. Hence, we have Pl-Holant $\left(\neq_{2} \mid\left[\hat{f}_{0}, \hat{f}_{1}, 1,0,0\right]\right) \leq_{T} \operatorname{Pl}-\operatorname{Holant}\left(\neq_{2} \mid \hat{f},=_{n}\right)$. Note that $\operatorname{Pl}$-Holant $\left(\neq_{2} \mid\left[\hat{f}_{0}, \hat{f}_{1}, 1,0,0\right]\right)$ is equivalent to Pl-Holant $\left(\neq_{2} \mid[0,0,1,0,0]\right)$. This can be seen as follows. From the $\neq_{2}$ on the LHS, any edge assignment must assign 0's and 1's to exactly half of the edges. However, considering from the RHS, if any occurrence of the signature $\left[\hat{f}_{0}, \hat{f}_{1}, 1,0,0\right]$ is assigned more 0 's than 1 's, then some other occurrence must be assigned more 1's than 0's which results in a global factor 0 . Thus each occurrence of the signature $\left[\hat{f}_{0}, \hat{f}_{1}, 1,0,0\right]$ is assigned exactly two 0 's and two 1 's, which is equivalent to $[0,0,1,0,0]$. The problem $\operatorname{Pl-Holant}\left(\neq_{2} \mid[0,0,1,0,0]\right)$ is counting Eulerian Orientations in planar 4-regular graphs. This problem was proved \#P-hard for general 4-regular graphs by Huang and Lu [27] and improved to planar 4-regular graphs by Guo and Williams [26]. It is also a consequence of Corollary 2.29. Thus Pl-Holant $\left(\neq_{2} \mid \hat{f},=_{n}\right)$ is \#P-hard as well.

\section{Dichotomy for PI-\#CSP² and Related Lemmas}

In this section, we state the dichotomy for Pl-\#CSP${ }^{2}$. We defer the proof to Part II of this paper, where we will restate it as Theorem 9.2. In this section we provide a sketch of the proof. Afterwards, we discuss several related lemmas, which are used for the full dichotomy of Pl-Holant. Let $\mathcal{T}_{k}=\left\{\left[\begin{array}{ll}1 & 0 \\ 0 & \theta\end{array}\right] \in \mathbb{C}^{2 \times 2} \mid \theta^{k}=1\right\}$.

Theorem 5.1 Let $\mathcal{F}$ be a set of symmetric signatures. Then $\operatorname{Pl} \# \operatorname{CSP}^{2}(\mathcal{F})$ is $\# P$-hard unless $\mathcal{F}$ satisfies one of the following conditions:

1. there exists $T \in \mathcal{T}_{8}$ such that $\mathcal{F} \subseteq T \mathscr{A}$;

2. $\mathcal{F} \subseteq \mathscr{P}$; 
3. there exists $T \in \mathcal{T}_{4}$ such that $\mathcal{F} \subseteq T \widehat{\mathscr{M}}$.

In each exceptional case, $\mathrm{Pl}-\# \mathrm{CSP}^{2}(\mathcal{F})$ is computable in polynomial time.

Proof Sketch We first define some tractable families of signatures specific to the Pl-\#CSP ${ }^{2}$ framework. Let $\tilde{\mathscr{A}}=\mathscr{A} \cup\left[\begin{array}{ll}1 & 0 \\ 0 & e^{\pi i / 4}\end{array}\right] \mathscr{A}$ and $\widetilde{\mathscr{M}}=\widehat{\mathscr{M}} \cup\left[\begin{array}{ll}1 & 0 \\ 0 & i\end{array}\right] \widehat{\mathscr{M}}$. One can show that $\tilde{\mathscr{A}}$ covers Case 1 above, and $\widetilde{\mathscr{M}}$ covers Case 3 . The proof will revolve around these tractable classes.

The overall plan is to break the proof into two main steps.

The first step is to prove the dichotomy theorem for $\operatorname{Pl}^{-\# C S P}{ }^{2}(\mathcal{F})$ when there is at least one nonzero signature of $o d d$ arity in $\mathcal{F}$. In this case, we can make use of a lemma showing that we can simulate $\operatorname{Pl}-\# \operatorname{CSP}(\mathcal{F})$ by $\operatorname{Pl}-\# \operatorname{CSP}^{2}(\mathcal{F})$ if $\mathcal{F}$ includes a unary signature $[a, b]$ with $a b \neq 0$. Then we can apply the known dichotomy Theorem 2.25 for Pl-\#CSP. However this strategy (provably) cannot work when every signature in $\mathcal{F}$ satisfies the parity constraint. In that case we employ other means. This first step of the proof is relatively uncomplicated.

The second step is to deal with the case when all nonzero signatures in $\mathcal{F}$ have even arity. This is where the real difficulties lie. In this case it is impossible to directly construct any unary signature. So we cannot use that lemma pertaining to a unary signature. But we prove another lemma which provides a way to simulate $\operatorname{Pl}$-\# $\operatorname{CSP}(\mathcal{F})$ by $\mathrm{Pl}-\# \operatorname{CSP}^{2}(\mathcal{F})$ in a global fashion, if $\mathcal{F}$ includes some tensor power of the form $[a, b]^{\otimes 2}$ where $a b \neq 0$. Moreover, we have a lucky break (for the complexity of the proof) if $\mathcal{F}$ includes a signature that is in $\widehat{\mathscr{M}} \backslash(\mathscr{P} \cup \widetilde{\mathscr{A}})$. In this case, we can construct a special binary signature, and obtain $[1,1]^{\otimes 2}$ by interpolation. This proof uses some elementary properties of cyclotomic fields. This simplifies the proof greatly. For all other cases (when $\mathcal{F}$ has only even arity signatures), the proof gets going in earnest - we will attempt an induction on the arity of signatures.

The lowest arity of this induction will be 2 . We will try to reduce the arity to 2 whenever possible; however for many cases an arity reduction to 2 destroys the \#Phardness at hand. Therefore the true basis of this induction proof of $\mathrm{Pl}$-\#CSP${ }^{2}$ starts with arity 4. Consequently we will first prove a dichotomy theorem for $\mathrm{Pl}-\# \mathrm{CSP}^{2}(f)$, where $f$ is a signature of arity 4 . Several tools will be used. These include the rank criterion for redundant signatures, Theorem 2.24 for arity 2 signatures, and a trick we call the Three Stooges by domain pairing.

However, in the next step we do not attempt a general $\mathrm{Pl}$-\#CSP ${ }^{2}$ dichotomy for a single signature of even arity. This would have been natural at this point, but it would have been too difficult. We will need some additional leverage by proving a conditional "No-Mixing" Lemma for pairs of signatures of even arity. So, seemingly taking a detour, we prove that for two signatures $f$ and $g$ both of even arity, that individually belong to some tractable class, but do not belong to a single tractable class in the conjectured dichotomy (that is yet to be proved), the problem $\mathrm{Pl}-\# \mathrm{CSP}^{2}(f, g)$ is \#P-hard. We prove this No-Mixing Lemma for any pair of signatures $f$ and $g$ both of even arity, not restricted to arity 4 . Even though at this point we only have a dichotomy for a single signature of arity 4, we prove this No-Mixing Lemma for higher even arity pairs $f$ and $g$ by simulating two signatures $f^{\prime}$ and $g^{\prime}$ of arity 4 that 
belong to different tractable sets, from that of $\mathrm{Pl}-\# \mathrm{CSP}^{2}(f, g)$. After this arity reduction (within the No-Mixing Lemma), we prove that $\mathrm{Pl}$ \#CSP${ }^{2}\left(f^{\prime}, g^{\prime}\right)$ is \#P-hard by the dichotomy for a single signature of arity 4. After this, we prove a No-Mixing Lemma for a set of signatures $\mathcal{F}$ of even arities, which states that if $\mathcal{F}$ is contained in the union of all tractable classes, then it is still \#P-hard unless it is entirely contained in one single tractable class. Note that at this point we still only have a conditional No-Mixing Lemma in the sense that we have to assume every signature in $\mathcal{F}$ belongs to some tractable set.

We then attempt the proof of a Pl-\#CSP ${ }^{2}$ dichotomy for a single signature of arbitrary even arity. This uses all the previous lemmas, in particular the (conditional) No-Mixing Lemma for a set of signatures. However, after completing the proof of this Pl-\#CSP ${ }^{2}$ dichotomy for a single signature of even arity, the No-Mixing Lemma becomes absolute.

Finally the dichotomy for a single signature of even arity is logically extended to a dichotomy theorem for $\operatorname{Pl}-\# \operatorname{CSP}^{2}(\mathcal{F})$ where all signatures in $\mathcal{F}$ have even arity. Together with the first main step when $\mathcal{F}$ contains some nonzero signature of odd arity, this completes the proof of Theorem 5.1.

\subsection{Related Lemmas}

Now we give some consequences of Theorem 5.1. These are cases that can be reduced to Pl-\#CSP ${ }^{2}$. We consider signatures in $\mathscr{P}_{1}, \mathscr{M}_{2} \backslash \mathscr{P}_{2}, \mathscr{A}_{3}$, or $\mathscr{M}_{3}$.

All signatures stated in lemmas and corollaries in this section are assumed symmetric. We begin with the cases of $\mathscr{P}_{1}$ and $\mathscr{A}_{3}$. The proofs of the following two lemmas are contained in the proofs of Lemma 61 and Lemma 63 in [11] respectively. One can check that the reductions in these proofs are planar.

Lemma 5.2 (cf. Lemma 61, p. 1717 in [11]) Let $f \in \mathscr{P}_{1}$ be a non-degenerate signature of arity $n \geq 3$ with an orthogonal transformation $H^{-1}$ and $\mathcal{F}$ be a set of signatures containing $f$. Let $H_{2}$ be the 2-by-2 matrix $\frac{1}{\sqrt{2}}\left[\begin{array}{ll}1 & 1 \\ 1 & -1\end{array}\right]$. Then $\operatorname{Pl}-\# \operatorname{CSP}^{2}\left(H_{2} H \mathcal{F}\right) \leq_{T} \operatorname{Pl-Holant}(\mathcal{F})$.

Lemma 5.3 (cf. Lemma 63, p. 1719 in [11]) Let $f \in \mathscr{A}_{3}$ be a non-degenerate signature of arity $n \geq 3$ with an orthogonal transformation $H^{-1}$ and $\mathcal{F}$ be a set of signatures containing $f$. Let $\alpha=e^{\pi i / 4}$ and $Y$ be the 2-by-2 matrix $\left[\begin{array}{ll}\alpha & 1 \\ -\alpha & 1\end{array}\right]$. Then $\operatorname{Pl-\# CSP}{ }^{2}(Y H \mathcal{F} \cup\{[1,-i, 1]\}) \leq_{T} \operatorname{Pl-Holant}(\mathcal{F})$.

With these reductions, we can apply Theorem 5.1 to get the following corollaries. Corollary 5.4 follows directly from Lemma 5.2 and Theorem 5.1 as $H_{2}$ is orthogonal and every $\mathrm{Pl}-\# \mathrm{CSP}^{2}$ tractable case is also tractable for Pl-Holant.

Corollary 5.4 Let $\mathcal{F}$ be a set of symmetric signatures. Suppose there exists $f \in$ $\mathcal{F}$ that is a non-degenerate signature of arity $n \geq 3$ in $\mathscr{P}_{1}$. Then $\operatorname{Pl-Holant}(\mathcal{F})$ is 
\#P-hard unless $\mathcal{F}$ is $\mathscr{A}$-, $\mathscr{P}$-, or $\mathscr{M}$-transformable, in which case $\operatorname{Pl-Holant}(\mathcal{F})$ is tractable.

The proof of this corollary is straightforward. To illustrate the power of Theorem 5.1, we give a short proof here.

Proof Suppose $f \in \mathscr{P}_{1}$. Then there is some $H \in \mathbf{O}_{2}(\mathbb{C})$ such that $\left(H_{2} H\right)^{\otimes n} f$ has the form $[a, 0, \ldots, 0, b]$, where $a b \neq 0$, and $H_{2}=\frac{1}{\sqrt{2}}\left[\begin{array}{ll}1 & 1 \\ 1 & -1\end{array}\right]$. Let $H^{\prime}=$ $\left(\mathrm{H}_{2} H\right)^{-1} \in \mathbf{O}_{2}(\mathbb{C})$. By Lemma 5.2 and Theorem 5.1, Pl-Holant $(\mathcal{F})$ is \#P-hard unless either (1) $\mathcal{F} \subseteq H^{\prime} \mathscr{P}$, or (2) $\mathcal{F} \subseteq H^{\prime} T \mathscr{A}$, or (3) $\mathcal{F} \subseteq H^{\prime} T^{\prime}\left[\begin{array}{ll}1 & 1 \\ 1 & -1\end{array}\right] \mathscr{M}$, where $T \in \mathcal{T}_{8}$ and $T^{\prime} \in \mathcal{T}_{4}$. In case $(1), \mathcal{F}$ is $\mathscr{P}$-transformable since $(=2) H^{\prime \otimes 2}=\left(=_{2}\right.$ )$\in \mathscr{P}$. In case $(2), \mathcal{F}$ is $\mathscr{A}$-transformable since $(=2)\left(H^{\prime} T\right)^{\otimes 2}=(=2) T^{\otimes 2} \in \mathscr{A}$. In case (3), $\mathcal{F}$ is $\mathscr{M}$-transformable. If $T^{\prime}=\left[\begin{array}{ll}1 & 0 \\ 0 & \pm 1\end{array}\right]$, then $T^{\prime} \in \mathbf{O}_{2}(\mathbb{C})$. So $(=2$ $\left(H^{\prime} T^{\prime}\left[\begin{array}{ll}1 & 1 \\ 1 & -1\end{array}\right]\right)^{\otimes 2}$ is unchanged $(=2) \in \mathscr{M}$. If $T^{\prime}=\left[\begin{array}{ll}1 & 0 \\ 0 & \pm i\end{array}\right]$, then $T^{\prime}\left[\begin{array}{ll}1 & 1 \\ 1 & -1\end{array}\right]=$ $\left[\begin{array}{ll}1 & 1 \\ i & -i\end{array}\right]$ or $\left[\begin{array}{ll}1 & 1 \\ -i & i\end{array}\right]$, and $(=2)\left(H^{\prime} T^{\prime}\left[\begin{array}{ll}1 & 1 \\ 1 & -1\end{array}\right]\right)^{\otimes 2}=2[0,1,0] \in \mathscr{M}$.

Corollary 5.4 is useful in Section 8. In Section 6, we need the following further specialization.

Corollary 5.5 Suppose $f$ is a non-degenerate signature of arity $n \geq 5$. Let $f^{\prime}$ be obtained from $f$ with a self loop, and assume that $f^{\prime} \in \mathscr{P}_{1}$ is non-degenerate. Then Pl-Holant $(f)$ is \#P-hard unless $f$ is $\mathscr{A}$-, $\mathscr{P}$-, or $\mathscr{M}$-transformable, in which case Pl-Holant $(f)$ is tractable.

For the case of $\mathscr{A}_{3}$, some case analysis is required.

Corollary 5.6 Let $\mathcal{F}$ be a set of signatures. Suppose there exists $f \in \mathcal{F}$ that is a non-degenerate signature of arity $n \geq 3$ in $\mathscr{A}_{3}$. Then $\mathrm{Pl}-\operatorname{Holant}(\mathcal{F})$ is \#P-hard unless $\mathcal{F}$ is $\mathscr{A}$ - or $\mathscr{M}$-transformable, in which case $\operatorname{Pl-Holant}(\mathcal{F})$ is tractable.

Proof Assume that $f \in \mathscr{A}_{3}$ with an orthogonal transformation $H^{-1}$. By Lemma 5.3, we have $\mathrm{Pl}-\# \mathrm{CSP}^{2}(Y H \mathcal{F} \cup\{[1,-i, 1]\}) \leq_{T} \operatorname{Pl-Holant}(\mathcal{F})$, where $Y=\left[\begin{array}{ll}\alpha & 1 \\ -\alpha & 1\end{array}\right]$ and $\alpha=e^{\pi i / 4}$. Let $g=[1,-i, 1]$ and $\mathcal{F}^{\prime}=Y H \mathcal{F} \cup\{g\}$.

We apply Theorem 5.1 to $\mathrm{Pl}-\# \operatorname{CSP}^{2}\left(\mathcal{F}^{\prime}\right)$. The consequence is that $\mathrm{Pl}-\# \operatorname{CSP}^{2}\left(\mathcal{F}^{\prime}\right)$ (and hence Pl-Holant $(F)$ ) is \#P-hard unless $\mathcal{F}^{\prime} \subseteq \mathscr{P}$, or $\mathcal{F}^{\prime} \subseteq\left[\begin{array}{ll}1 & 0 \\ 0 & i^{r}\end{array}\right] \widehat{\mathscr{M}}$ for some integer $0 \leq r \leq 3$, or $\mathcal{F}^{\prime} \subseteq\left[\begin{array}{ll}1 & 0 \\ 0 & \alpha^{r}\end{array}\right] \mathscr{A}$ for some integer $0 \leq r \leq 7$ where $\alpha=e^{i \pi / 4}$. Notice that $g \notin \mathscr{P}$ and hence the first case is impossible. 
Suppose $\mathcal{F}^{\prime} \subseteq\left[\begin{array}{ll}1 & 0 \\ 0 & i^{r}\end{array}\right] \widehat{\mathscr{M}}$ for some integer $0 \leq r \leq 3$. It is easy to verify that binary symmetric signatures in $\widehat{\mathscr{M}}$ have the form $[a, b, a]$ or $[a, 0,-a]$. However $\left[\begin{array}{ll}1 & 0 \\ 0 & i^{r}\end{array}\right]^{-1} g$ has the matrix form $\left[\begin{array}{ll}1 & 0 \\ 0 & i^{-r}\end{array}\right]\left[\begin{array}{ll}1 & -i \\ -i & 1\end{array}\right]\left[\begin{array}{ll}1 & 0 \\ 0 & i^{-r}\end{array}\right]^{\mathrm{T}}=$ $\left[\begin{array}{ll}1 & -i^{1-r} \\ -i^{1-r} & (-1)^{-r}\end{array}\right]$. For $r=1,3$, this is not in $\widehat{\mathscr{M}}$. So $g \notin\left[\begin{array}{ll}1 & 0 \\ 0 & i^{r}\end{array}\right] \widehat{\mathscr{M}}$ for $r=1,3$, and we have that $Y H \mathcal{F} \subseteq\left[\begin{array}{ll}1 & 0 \\ 0 & \pm 1\end{array}\right] \widehat{\mathscr{M}}$. Moreover, notice that $\left[\begin{array}{ll}1 & 0 \\ 0 & -1\end{array}\right] \widehat{\mathscr{M}}=$ $\left[\begin{array}{ll}1 & 1 \\ 1 & -1\end{array}\right]\left[\begin{array}{ll}0 & 1 \\ 1 & 0\end{array}\right] \mathscr{M}=\left[\begin{array}{ll}1 & 1 \\ 1 & -1\end{array}\right] \mathscr{M}=\widehat{\mathscr{M}}$. Hence $Y H \mathcal{F} \subseteq \widehat{\mathscr{M}}$. Rewrite $Y$ as $Y=\left[\begin{array}{ll}1 & 1 \\ -1 & 1\end{array}\right]\left[\begin{array}{ll}\alpha & 0 \\ 0 & 1\end{array}\right]$. We deduce that

$$
\begin{gathered}
H \mathcal{F} \subseteq \frac{1}{2}\left[\begin{array}{ll}
\alpha^{-1} & 0 \\
0 & 1
\end{array}\right]\left[\begin{array}{ll}
1 & -1 \\
1 & 1
\end{array}\right] \widehat{\mathscr{M}}=\frac{1}{2}\left[\begin{array}{ll}
\alpha^{-1} & 0 \\
0 & 1
\end{array}\right]\left[\begin{array}{ll}
1 & -1 \\
1 & 1
\end{array}\right]\left[\begin{array}{ll}
1 & 1 \\
1 & -1
\end{array}\right] \mathscr{M} \\
=\left[\begin{array}{ll}
\alpha^{-1} & 0 \\
0 & 1
\end{array}\right]\left[\begin{array}{ll}
0 & 1 \\
1 & 0
\end{array}\right] \mathscr{M}=\mathscr{M},
\end{gathered}
$$

using Proposition 3.6. Hence $\mathcal{F}$ is $\mathscr{M}$-transformable in this case.

The last case is when $\mathcal{F}^{\prime} \subseteq\left[\begin{array}{ll}1 & 0 \\ 0 & \alpha^{r}\end{array}\right] \mathscr{A}$ for some integer $0 \leq r \leq 7$. The matrix form of the signature $g$ is $\left[\begin{array}{ll}1 & -i \\ -i & 1\end{array}\right]$. We note that $\left[\begin{array}{ll}1 & 0 \\ 0 & \alpha^{-1}\end{array}\right]^{\mathrm{T}}\left[\begin{array}{ll}1 & -i \\ -i & 1\end{array}\right]\left[\begin{array}{ll}1 & 0 \\ 0 & \alpha^{-1}\end{array}\right]=$ $\left[\begin{array}{ll}1 & -i \alpha^{-1} \\ -i \alpha^{-1}-i\end{array}\right] \notin \mathscr{A}$ as the entries are not powers of $i$, so $g \notin\left[\begin{array}{ll}1 & 0 \\ 0 & \alpha\end{array}\right] \mathscr{A}$. Notice that $\left[\begin{array}{ll}1 & 0 \\ 0 & i^{l}\end{array}\right] \in \operatorname{Stab}(\mathscr{A})$. Since $g \in\left[\begin{array}{ll}1 & 0 \\ 0 & \alpha^{r}\end{array}\right] \mathscr{A}$, we get $r=0,2,4,6$. That is, $\mathcal{F}^{\prime} \subseteq\left[\begin{array}{ll}1 & 0 \\ 0 & i^{l}\end{array}\right] \mathscr{A}$ for some integer $0 \leq l \leq 3$. This implies that $Y H \mathcal{F} \subseteq \mathscr{A}$. Again, rewriting $Y$ as $Y=\left[\begin{array}{ll}1 & 1 \\ -1 & 1\end{array}\right]\left[\begin{array}{ll}\alpha & 0 \\ 0 & 1\end{array}\right]$, we have

$$
H \mathcal{F} \subseteq \frac{1}{2}\left[\begin{array}{ll}
\alpha^{-1} & 0 \\
0 & 1
\end{array}\right]\left[\begin{array}{ll}
1 & -1 \\
1 & 1
\end{array}\right] \mathscr{A}=\frac{1}{2}\left[\begin{array}{ll}
\alpha^{-1} & 0 \\
0 & 1
\end{array}\right] \mathscr{A}
$$

Therefore $\mathcal{F}$ is $\mathscr{A}$-transformable. This finishes the proof.

Again, we specialize Corollary 5.6 to our need.

Corollary 5.7 Let $f$ be a non-degenerate signature of arity $n \geq 5$. Let $f^{\prime}$ be obtained from $f$ with a self loop. If $f^{\prime}$ is non-degenerate and $f^{\prime} \in \mathscr{A}_{3}$, then $\operatorname{Pl-Holant}(f)$ is \#P-hard unless $f$ is $\mathscr{A}$-or $\mathscr{M}$-transformable, in which case Pl-Holant $(f)$ is tractable. 
The next case is when $f$ is in $\mathscr{M}_{2}$ but not $\mathscr{P}_{2}$.

Lemma 5.8 Let $\mathcal{F}$ be a set of signatures. Suppose there exists $f \in \mathcal{F}$ which is a non-degenerate signature of arity $n \geq 3$ in $\mathscr{M}_{2} \backslash \mathscr{P}_{2}$. Then $\mathrm{Pl}$-Holant $(\mathcal{F})$ is $\# P$-hard unless $\mathcal{F}$ is $\mathscr{A}$-, $\mathscr{P}_{-}$, or $\mathscr{M}$-transformable, in which case Pl-Holant $(\mathcal{F})$ is tractable.

Proof As $f \in \mathscr{M}_{2} \backslash \mathscr{P}_{2}$, we may assume $f=H^{\otimes n}\left(\left[\begin{array}{l}1 \\ \gamma\end{array}\right]^{\otimes n} \pm\left[\begin{array}{l}1 \\ -\gamma\end{array}\right]^{\otimes n}\right)$ up to a nonzero constant, where $H$ is an orthogonal 2-by-2 matrix and $\gamma \neq 0, \pm i$.

We first show that

$$
\operatorname{Pl-\# CSP}{ }^{2}\left(T^{-1} \mathcal{F}, g\right) \leq_{T} \operatorname{Pl-Holant}(f, \mathcal{F}),
$$

where $T=H\left[\begin{array}{ll}1 & 1 \\ \gamma & -\gamma\end{array}\right]$ and $g=(=2) T^{\otimes 2}=\left[1+\gamma^{2}, 1-\gamma^{2}, 1+\gamma^{2}\right]$.

Assume that $f=H^{\otimes n}\left(\left[\begin{array}{l}1 \\ \gamma\end{array}\right]^{\otimes n}+\left[\begin{array}{l}1 \\ -\gamma\end{array}\right]^{\otimes n}\right)$ with the + sign. In this case, we do the transformation $T$ :

$$
\begin{aligned}
\text { Pl-Holant }(=2 \mid f, \mathcal{F}) & \equiv_{T} \text { Pl-Holant }\left([1,0,1] H^{\otimes 2}\left[\begin{array}{ll}
1 & 1 \\
\gamma & -\gamma
\end{array}\right]^{\otimes 2} \mid\left(\left[\begin{array}{ll}
1 & 1 \\
\gamma & -\gamma
\end{array}\right]^{-1}\right)^{\otimes n}\left(H^{-1}\right)^{\otimes n} f, T^{-1} \mathcal{F}\right) \\
& \equiv_{T} \text { Pl-Holant }\left(g \mid={ }_{n}, T^{-1} \mathcal{F}\right) .
\end{aligned}
$$

By connecting $g$ to two inputs of $={ }_{n}$, we get $={ }_{n-2}$ up to a constant factor of $1+\gamma^{2} \neq$ 0 as $\gamma \neq \pm i$. We repeat this process. If $n$ is even, then we get $=2$ eventually, which is on the right hand side in the above Pl-Holant problem. If $n$ is odd, then eventually we get $=3$ and $(=1)=[1,1]$ on the right. Connecting $[1,1]$ to $g$ we get $2[1,1]$ on the left. Then connecting $[1,1]$ to $={ }_{3}$ we get $={ }_{2}$ on the right. To summarize, we get that

$$
\begin{aligned}
\text { Pl-Holant }\left(g \mid={ }_{2},=_{n}, T^{-1} \mathcal{F}\right) & \leq{ }_{T} \operatorname{Pl-Holant}\left(g \mid={ }_{n}, T^{-1} \mathcal{F}\right) \\
& \leq_{T} \operatorname{Pl-Holant}(f, \mathcal{F}) .
\end{aligned}
$$

Next we show that

$$
\text { Pl-Holant }\left(={ }_{2}, g \mid=2,={ }_{n}, T^{-1} \mathcal{F}\right) \leq_{T} \text { Pl-Holant }\left(g \mid={ }_{2},={ }_{n}, T^{-1} \mathcal{F}\right) \text {. }
$$

Let $N=\left[\begin{array}{ll}1+\gamma^{2} & 1-\gamma^{2} \\ 1-\gamma^{2} & 1+\gamma^{2}\end{array}\right]$ be the signature matrix of $g$. If there is a positive integer $k$ and a nonzero constant $c$ such that $N^{k}=c I_{2}$, where $I_{2}$ is the 2-by-2 identity matrix, then we may directly implement $=2$ on the left by connecting $k$ copies of $\left[1+\gamma^{2}, 1-\gamma^{2}, 1+\gamma^{2}\right]$ via $={ }_{2}$ on the right. This implies (5.6) holds.

Otherwise such $k$ and $c$ do not exist. The two eigenvalues of $N$ are $\lambda_{1}=2$ and $\lambda_{2}=2 \gamma^{2}$. If $\lambda_{1}=\lambda_{2}$, then $\gamma^{2}=1$ and $N=\left[\begin{array}{ll}2 & 0 \\ 0 & 2\end{array}\right]$. Contradiction. Hence $\lambda_{1} \neq \lambda_{2}$, and $N$ is diagonalizable. Let $N=P\left[\begin{array}{ll}\lambda_{1} & 0 \\ 0 & \lambda_{2}\end{array}\right] P^{-1}$, for some non-singular matrix $P$. By connecting $l$ many copies of $N$ on the left via $=2$ on the right, where $l$ is a positive 
integer, we can implement $N^{l}=P\left[\begin{array}{ll}\lambda_{1}^{l} & 0 \\ 0 & \lambda_{2}^{l}\end{array}\right] P^{-1}$. Since $N$ does not have finite order up to a scalar, for any positive integer $l,\left(\lambda_{1} / \lambda_{2}\right)^{l} \neq 1$.

Consider an instance $\Omega$ of Pl-Holant $\left(=_{2}, g \mid=_{2},=_{n}, T^{-1} \mathcal{F}\right)$. Suppose that the left $=2$ appears $t$ times. Let $l$ be a positive integer. We obtain $\Omega_{l}$ from $\Omega$ by replacing each occurrence of $={ }_{2}$ on the left with $N^{l}$.

Since $N^{l}=P\left[\begin{array}{ll}\lambda_{1}^{l} & 0 \\ 0 & \lambda_{2}^{l}\end{array}\right] P^{-1}$, we can view our construction of $\Omega_{l}$ as replacing $N^{l}$ by 3 signatures, with matrix $P,\left[\begin{array}{ll}\lambda_{1}^{l} & 0 \\ 0 & \lambda_{2}^{l}\end{array}\right]$, and $P^{-1}$, respectively. This does not change the Holant value.

We stratify the assignments in $\Omega_{l}$ based on the assignments to the $t$ occurrences of the signature whose matrix is the diagonal matrix $\left[\begin{array}{ll}\lambda_{1}^{l} & 0 \\ 0 & \lambda_{2}^{l}\end{array}\right]$. Suppose there are $i$ many times it was assigned 00 with function value $\lambda_{1}^{l}$, and $j$ times 11 with function value $\lambda_{2}^{l}$. Clearly $i+j=t$ if the assignment has a nonzero evaluation. Let $c_{i j}$ be the sum over all such assignments of the products of evaluations of all signatures (including the signatures corresponding to matrices $P$ and $P^{-1}$ ) in $\Omega_{l}$ except for this diagonal one. Then

$$
\text { Holant }_{\Omega_{l}}=\sum_{i+j=t}\left(\lambda_{1}^{l}\right)^{i}\left(\lambda_{2}^{l}\right)^{j} c_{i j}=\lambda_{2}^{l t} \sum_{0 \leq i \leq t}\left(\left(\frac{\lambda_{1}}{\lambda_{2}}\right)^{l}\right)^{i} c_{i, t-i}
$$

By an oracle for Pl-Holant $\left(g \mid=2,={ }_{n}, T^{-1} \mathcal{F}\right)$, we can get Holant $\Omega_{l}$ for any $1 \leq$ $l \leq t+1$. Recall that for any positive integer $l,\left(\lambda_{1} / \lambda_{2}\right)^{l} \neq 1$. This implies that for any two distinct integers $i, j \geq 0,\left(\lambda_{1} / \lambda_{2}\right)^{i} \neq\left(\lambda_{1} / \lambda_{2}\right)^{j}$. Therefore we get a nonsingular Vandermonde system. We can solve all $c_{i j}$ for $i+j=t$ given Holant $\Omega_{l}$ for all $1 \leq l \leq t+1$. Then notice that $\sum_{i+j=t} c_{i j}$ is the Holant value of $\Omega_{l}$ by replacing both $\lambda_{1}^{l}$ and $\lambda_{2}^{l}$ with 1 , which is the instance $\Omega$ as $P I_{2} P^{-1}=I_{2}$. Therefore we may compute Holant $\Omega_{\Omega}$ via $t+1$ many oracle calls to Pl-Holant $\left(g \mid={ }_{2},=_{n}, T^{-1} \mathcal{F}\right)$. This finishes the reduction in (5.6).

The problem Pl-Holant $\left(=2, g \mid={ }_{2},={ }_{n}, T^{-1} \mathcal{F}\right)$ in the left hand side of (5.6) has $=2$ on both sides. Therefore we may lift the bipartite restriction. Combining it with (5.5), we get

$$
\operatorname{Pl-Holant}\left(=_{n}, g, T^{-1} \mathcal{F}\right) \leq_{T} \operatorname{Pl-Holant}(f, \mathcal{F}) \text {. }
$$

Notice that given an equality of arity $n \geq 3$, we can always construct all equalities of even arity, regardless of the parity of $n$, in the Pl-Holant setting. Therefore, we have

$$
\operatorname{Pl-\# CSP}{ }^{2}\left(T^{-1} \mathcal{F}, g\right) \leq_{T} \text { Pl-Holant }(f, \mathcal{F}),
$$

finishing the proof of (5.4) in the case $f=H^{\otimes n}\left(\left[\begin{array}{l}1 \\ \gamma\end{array}\right]^{\otimes n}+\left[\begin{array}{l}1 \\ -\gamma\end{array}\right]^{\otimes n}\right)$. 
To complete the proof of (5.4), there is another case that $f=$ $H^{\otimes n}\left(\left[\begin{array}{l}1 \\ \gamma\end{array}\right]^{\otimes n}-\left[\begin{array}{l}1 \\ -\gamma\end{array}\right]^{\otimes n}\right)$, with the - sign. Again we do a $T$ transformation, where $\left(T^{-1}\right)^{\otimes} f=[1,0, \ldots, 0,-1]$ has arity $n$ :

$$
\text { Pl-Holant }(=2 \mid f, \mathcal{F}) \equiv_{T} \text { Pl-Holant }\left(g \mid[1,0, \ldots, 0,-1], T^{-1} \mathcal{F}\right) .
$$

We then do the same construction as in the previous case of connecting $g$ to $[1,0, \ldots, 0,-1]$ repeatedly. Depending on the parity of $n$, we have two cases.

1. If $n$ is odd, then eventually we get $[1,0,0,-1]$ and $[1,-1]$ on the right as $\gamma \neq$ $\pm i$, and therefore $2 \gamma^{2}[1,-1]$, i.e., $[1,-1]$ on the left as $\gamma \neq 0$. Then connecting $[1,-1]$ to $[1,0,0,-1]$ we get $=2$ on the right. Thus, for odd $n$,

$$
\begin{gathered}
\text { Pl-Holant }\left(g \mid={ }_{2},[1,0, \ldots, 0,-1], T^{-1} \mathcal{F}\right) \leq_{T} \text { Pl-Holant }\left(g \mid[1,0, \ldots, 0,-1], T^{-1} \mathcal{F}\right) \\
\leq_{T} \text { Pl-Holant }(f, \mathcal{F}) .
\end{gathered}
$$

Notice that our previous binary interpolation proof only relies on $g$ and $=_{2}$. Hence we get

$$
\begin{aligned}
\text { Pl-Holant }\left(g \mid={ }_{2},[1,0, \ldots, 0,-1], T^{-1} \mathcal{F}\right) & \geq_{T} \text { Pl-Holant }\left(={ }_{2}, g \mid={ }_{2},[1,0, \ldots, 0,-1], T^{-1} \mathcal{F}\right) \\
& \equiv_{T} \text { Pl-Holant }\left([1,0, \ldots, 0,-1], g, T^{-1} \mathcal{F}\right) .
\end{aligned}
$$

Moreover it is straightforward to construct all even equalities from $[1,0, \ldots, 0,-1]$ of arity $n$ in the normal Pl-Holant setting as $n \geq 5$. Combining everything together gives us

$$
\operatorname{Pl} \# \operatorname{CSP}^{2}\left(g, T^{-1} \mathcal{F}\right) \leq_{T} \text { Pl-Holant }(f, \mathcal{F}) .
$$

2. Otherwise $n$ is even. By the same construction of connecting $g$ to $[1,0, \ldots, 0,-1]$ repeatedly, we get $[1,0,0,0,-1]$ and $[1,0,-1]$ on the right eventually. Then we connect two copies of $g$ via $[1,0,-1]$, resulting in $\left[\begin{array}{ll}1+\gamma^{2} & 1-\gamma^{2} \\ 1-\gamma^{2} & 1+\gamma^{2}\end{array}\right]\left[\begin{array}{ll}1 & 0 \\ 0 & -1\end{array}\right]\left[\begin{array}{ll}1+\gamma^{2} & 1-\gamma^{2} \\ 1-\gamma^{2} & 1+\gamma^{2}\end{array}\right]=4 \gamma^{2}\left[\begin{array}{ll}1 & 0 \\ 0 & -1\end{array}\right]$ on the left. Then connect $[1,0,-1]$ to $[1,0,0,0,-1]$ to get $[1,0,1]$ on the right. At last we connect two $[1,0,-1]$ 's on the left via $[1,0,1]$ on the right to get $[1,0,1]$ on the left. Then it reduces to the previous case.

This concludes the proof of (5.4).

We apply Theorem 5.1 to $\operatorname{Pl}-\# \operatorname{CSP}^{2}\left(T^{-1} \mathcal{F}, g\right)$. Then we have that $\mathrm{Pl}$-\#CSP${ }^{2}\left(T^{-1} \mathcal{F}, g\right)$ (and hence $\operatorname{Pl-Holant}(f, \mathcal{F})$ ) is \#P-hard unless $T^{-1} \mathcal{F} \cup\{g\} \subseteq$ $\mathscr{P}$, or $T^{-1} \mathcal{F} \cup\{g\} \subseteq\left[\begin{array}{ll}1 & 0 \\ 0 & i^{r}\end{array}\right] \widehat{\mathscr{M}}$ for some integer $0 \leq r \leq 3$, or $T^{-1} \mathcal{F} \cup\{g\} \subseteq\left[\begin{array}{ll}1 & 0 \\ 0 & \alpha^{r}\end{array}\right] \mathscr{A}$ for some integer $0 \leq r \leq 7$ where $\alpha=e^{i \pi / 4}$. We deal with these three cases. We start with the simplest case $\mathscr{P}$, then $\widehat{\mathscr{M}}$, and then $\mathscr{A}$.

1. The first case is that $T^{-1} \mathcal{F} \cup\{g\} \subseteq \mathscr{P}$. Recall that $\gamma \neq 0$ or $\pm i$, it can be verified that $g \notin \mathscr{P}$ unless $\gamma^{2}=1$. Hence $\gamma= \pm 1$. In either case we have that 
$\left[\begin{array}{ll}1 & 1 \\ \gamma & -\gamma\end{array}\right]$ is an orthogonal matrix up to a nonzero scalar, and hence so is $T$. This implies that $\mathcal{F}$ is $\mathscr{P}$-transformable.

2. Next suppose $T^{-1} \mathcal{F} \cup\{g\} \subseteq\left[\begin{array}{ll}1 & 0 \\ 0 & i^{r}\end{array}\right] \widehat{\mathscr{M}}$ for some integer $0 \leq r \leq 3$. If $\gamma= \pm 1$, then $T$ is an orthogonal matrix as $\left[\begin{array}{ll}1 & 1 \\ \gamma & -\gamma\end{array}\right]$ is, up to a factor of $\frac{1}{\sqrt{2}}$. Hence $\mathcal{F}$ is $\mathscr{M}$-transformable, as $\mathcal{F} \subseteq T\left[\begin{array}{ll}1 & 0 \\ 0 & i^{r}\end{array}\right]\left[\begin{array}{ll}1 & 1 \\ 1 & -1\end{array}\right] \mathscr{M}$ and $(=2)\left(T\left[\begin{array}{ll}1 & 0 \\ 0 & i^{r}\end{array}\right]\left[\begin{array}{ll}1 & 1 \\ 1 & -1\end{array}\right]\right)^{\otimes 2}$ is either $[1,0,1]$ when $r=0,2$, or $[0,1,0]$ when $r=1,3$, up to a nonzero factor.

Otherwise $\gamma^{2} \neq 1$ and it is straightforward to verify that $g \notin\left[\begin{array}{ll}1 & 0 \\ 0 & i^{r}\end{array}\right] \widehat{\mathscr{M}}$ for $r=1,3$. Hence we may assume that $T^{-1} \mathcal{F} \subseteq\left[\begin{array}{ll}1 & 0 \\ 0 & \pm 1\end{array}\right] \widehat{\mathscr{M}}$. Moreover, $\left[\begin{array}{ll}1 & 0 \\ 0 & -1\end{array}\right] \widehat{\mathscr{M}}=\left[\begin{array}{ll}1 & 1 \\ 1 & -1\end{array}\right]\left[\begin{array}{ll}0 & 1 \\ 1 & 0\end{array}\right] \mathscr{M}=\left[\begin{array}{ll}1 & 1 \\ 1 & -1\end{array}\right] \mathscr{M}=\widehat{\mathscr{M}}$. Then $T^{-1} \mathcal{F} \subseteq \widehat{\mathscr{M}}$. As $T^{-1}=\left[\begin{array}{ll}1 & 1 \\ \gamma & -\gamma\end{array}\right]^{-1} H^{-1}$, this implies that

$$
\begin{aligned}
H^{-1} \mathcal{F} & \subseteq\left[\begin{array}{ll}
1 & 1 \\
\gamma & -\gamma
\end{array}\right] \widehat{\mathscr{M}}=\left[\begin{array}{ll}
1 & 0 \\
0 & \gamma
\end{array}\right]\left[\begin{array}{ll}
1 & 1 \\
1 & -1
\end{array}\right]\left[\begin{array}{ll}
1 & 1 \\
1 & -1
\end{array}\right] \mathscr{M} \\
& =\left[\begin{array}{ll}
1 & 0 \\
0 & \gamma
\end{array}\right] \mathscr{M}=\mathscr{M}
\end{aligned}
$$

Hence $\mathcal{F} \subseteq H \mathscr{M}$ and $\mathcal{F}$ is $\mathscr{M}$-transformable.

3. In the last case, $T^{-1} \mathcal{F} \cup\{g\} \subseteq\left[\begin{array}{ll}1 & 0 \\ 0 & \alpha^{r}\end{array}\right] \mathscr{A}$ for some integer $0 \leq r \leq 7$. If $\gamma= \pm 1$, then $T$ is an orthogonal matrix as $\left[\begin{array}{ll}1 & 1 \\ \gamma & -\gamma\end{array}\right]$ is, up to a factor of $\frac{1}{\sqrt{2}}$. Hence $\mathcal{F}$ is $\mathscr{A}$-transformable, as $\mathcal{F} \subseteq T\left[\begin{array}{ll}1 & 0 \\ 0 & \alpha^{r}\end{array}\right] \mathscr{A}$ and $(=2)\left(T\left[\begin{array}{ll}1 & 0 \\ 0 & \alpha^{r}\end{array}\right]\right)^{\otimes 2}$ is $\left[1,0, i^{r}\right] \in \mathscr{A}$, up to a nonzero factor.

Otherwise $\gamma^{2} \neq 1$ and $g \notin\left[\begin{array}{ll}1 & 0 \\ 0 & \alpha^{r}\end{array}\right] \mathscr{A}$ for any integer $r=1,3,5,7$. Hence $T^{-1} \mathcal{F} \cup\{g\} \subseteq \mathscr{A}$ as $\left[\begin{array}{ll}1 & 0 \\ 0 & i^{r}\end{array}\right] \mathscr{A}=\mathscr{A}$ for any integer $0 \leq r \leq 3$. If $\frac{1+\gamma^{2}}{1-\gamma^{2}} \neq \pm i$, then one can check that $g \notin \mathscr{A}$. A contradiction. Otherwise $\frac{1+\gamma^{2}}{1-\gamma^{2}}= \pm i$. This implies that $\gamma=\alpha^{l}$ for some integer $l=1,3,5,7$. We may assume $l=1$ as 
other cases are similar. In this case it is possible that $T^{-1} \mathcal{F} \cup\{g\} \subseteq \mathscr{A}$. As

$$
\begin{aligned}
& T^{-1}= {\left[\begin{array}{ll}
1 & 1 \\
\gamma & -\gamma
\end{array}\right]^{-1} H^{-1}=\left[\begin{array}{ll}
1 & 1 \\
\alpha & -\alpha
\end{array}\right]^{-1} H^{-1} \text {, this implies that } } \\
& H^{-1} \mathcal{F} \subseteq\left[\begin{array}{ll}
1 & 1 \\
\alpha & -\alpha
\end{array}\right] \mathscr{A}=\left[\begin{array}{ll}
1 & 0 \\
0 & \alpha
\end{array}\right]\left[\begin{array}{ll}
1 & 1 \\
1 & -1
\end{array}\right] \mathscr{A}=\left[\begin{array}{ll}
1 & 0 \\
0 & \alpha
\end{array}\right] \mathscr{A} .
\end{aligned}
$$

Hence, $\mathcal{F}$ is $\mathscr{A}$-transformable, so $\operatorname{Pl-Holant}(\mathcal{F})$ is tractable. This finishes the proof.

Lemma 5.8 leads to the following specialization.

Corollary 5.9 Let $f$ be a non-degenerate signature of arity $n \geq 5$. Let $f^{\prime}$ be $f$ with a self loop, and $f^{\prime}$ is non-degenerate and $f^{\prime} \in \mathscr{M}_{2} \backslash \mathscr{P}_{2}$. Then Pl-Holant $(f)$ is \#P-hard unless $f$ is $\mathscr{A}$-, $\mathscr{P}$-, or $\mathscr{M}$-transformable, in which case Pl-Holant $(f)$ is tractable.

We can reduce the case of $f \in \mathscr{M}_{3}$ to the previous case.

Lemma 5.10 Let $\mathcal{F}$ be a set of signatures. Suppose there exists $f \in \mathcal{F}$ that is a non-degenerate signature of arity $n \geq 3$ in $\mathscr{M}_{3}$. Then $\mathrm{Pl}-\operatorname{Holant}(\mathcal{F})$ is \#P-hard unless $\mathcal{F} \subseteq H \mathscr{M}$ for some $H \in \mathbf{O}_{2}(\mathbb{C})$, in which case $\mathcal{F}$ is $\mathscr{M}$-transformable and $\operatorname{Pl}$-Holant $(\mathcal{F})$ is tractable.

Proof We first claim that $\mathrm{Pl-Holant}(\mathcal{F})$ is \#P-hard unless $\mathcal{F}$ is $\mathscr{A}$-, $\mathscr{P}$-, or $\mathscr{M}$ transformable.

By the definition of $\mathscr{M}_{3}$, we may assume, up to a nonzero constant, that $\hat{f}=$ $\left(H^{-1}\right)^{\otimes n} f=$ EXACTONE $_{n}$ for some orthogonal matrix $H \in \mathbf{O}_{2}(\mathbb{C})$. After zero or more self loops, we can further assume that either $\hat{f}=\operatorname{EXACTONE}_{3}$ or $\hat{f}=$ EXACTONE 4 depending on the parity of $n$.

Suppose $\hat{f}=$ ExACTONE$_{3}$. Consider the gadget in Fig. 6a. We assign $\hat{f}$ to all vertices. The signature of the resulting gadget is $g=[0,1,0,1]$, which is in $\mathscr{M}_{2}$ and not in $\mathscr{P}_{2}=\mathscr{A}_{2}$ by Lemma 3.2. Thus, the claim follows from Lemma 5.8.

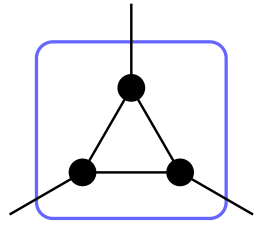

(a)

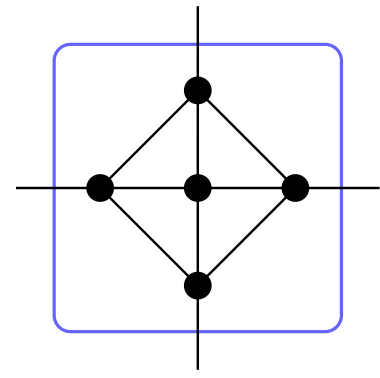

(b)

Fig. 6 Two gadgets used to create a signature in $\mathscr{M}_{2} \backslash \mathscr{P}_{2}$. a Triangle gadget. b Planar tetrahedron gadget 
Otherwise, $\hat{f}=$ EXACTONE$_{4}$. Consider the gadget in Figure $6 \mathrm{~b}$. We assign $\hat{f}$ to all vertices. Note that this is a matchgate. The signature of the resulting gadget is $[0,2,0,1,0]$, which is in $\mathscr{M}_{2}$ and not in $\mathscr{P}_{2}=\mathscr{A}_{2}$ by Lemma 3.2. Thus, the claim follows from Lemma 5.8. This completes the proof of the claim.

However, as $f \in \mathcal{F}$ and $f \in \mathscr{M}_{3}, \mathcal{F}$ cannot be $\mathscr{A}$ - or $\mathscr{P}$-transformable by Lemma 3.12. Also by Lemma 3.12, if $\mathcal{F}$ is $\mathscr{M}$-transformable, then $\mathcal{F} \subseteq H D \mathscr{M}$ or $H\left[\begin{array}{ll}0 & 1 \\ 1 & 0\end{array}\right] D \mathscr{M}$ for some diagonal matrix $D$. Notice that $D \in \operatorname{Stab}(\mathscr{M})$ and $\left[\begin{array}{ll}0 & 1 \\ 1 & 0\end{array}\right] D \in \operatorname{Stab}(\mathscr{M})$. This implies that $\mathcal{F} \subseteq H \mathscr{M}$.

Once again, we specialize Lemma 5.10 to our needs.

Corollary 5.11 Let $f$ be a non-degenerate signature of arity $n \geq 5$. Let $f^{\prime}$ be $f$ with a self loop, and $f^{\prime}$ is non-degenerate and $f^{\prime} \in \mathscr{M}_{3}$. Then Pl-Holant $(f)$ is \#P-hard unless $f$ is $\mathscr{M}$-transformable, in which case Pl-Holant $(f)$ is tractable.

\section{Single Signature Dichotomy}

Theorem 6.1 is the single signature dichotomy for Pl-Holant problems.

Theorem 6.1 If $f$ is a non-degenerate symmetric signature of arity $n \geq 3$ with complex weights in Boolean variables, then Pl-Holant $(f)$ is \#P-hard unless $f \in$ $\mathscr{P}_{1} \cup \mathscr{M}_{2} \cup \mathscr{A}_{3} \cup \mathscr{M}_{3} \cup \mathscr{M}_{4} \cup \mathscr{V}$, in which case the problem is computable in polynomial time.

Remark 2 The expression $f \in \mathscr{P}_{1} \cup \mathscr{M}_{2} \cup \mathscr{A}_{3} \cup \mathscr{M}_{3} \cup \mathscr{M}_{4} \cup \mathscr{V}$ has other equivalent forms. For $f$ of arity $n \geq 3$, if $f \in \mathscr{M}_{4}$ then $f \in \mathscr{V}$, and therefore we can also write the expression in Theorem 6.1 as $f \in \mathscr{P}_{1} \cup \mathscr{M}_{2} \cup \mathscr{A}_{3} \cup \mathscr{M}_{3} \cup \mathscr{V}$. However we retain the term $\mathscr{M}_{4}$ for convenience later. Indeed, we have $\mathscr{M}_{1} \subset \mathscr{A}_{1} \subset \mathscr{P}_{1}, \mathscr{A}_{2}=\mathscr{P}_{2} \subset \mathscr{M}_{2}$ (see Fig. 3). Thus the expression in Theorem 6.1 can also be written as

$$
f \in \mathscr{P}_{1} \cup \mathscr{P}_{2} \cup \mathscr{A}_{1} \cup \mathscr{A}_{2} \cup \mathscr{A}_{3} \cup \mathscr{M}_{1} \cup \mathscr{M}_{2} \cup \mathscr{M}_{3} \cup \mathscr{M}_{4} \cup \mathscr{V}
$$

In this form, the conclusion of Theorem 6.1 is clear: Pl-Holant $(f)$ is \#P-hard unless $f$ is $\mathscr{A}$ - or $\mathscr{P}$ - or $\mathscr{M}$-transformable or vanishing, by Lemmas $3.5,3.3,3.11$ and Theorem 2.15 .

We prove Theorem 6.1 by induction on the arity. Before proceeding to the proof, we first introduce several lemmas involved in the inductive step. All signatures stated in lemmas and corollaries in this section are assumed symmetric, unless indicated otherwise (e.g., the signature $\hat{g}$ in Lemma 6.7). 


\subsection{Lemmas Applied to Non-Degenerate Signatures in the Inductive Step}

The single signature dichotomy relies on the following key lemma. The important assumption here is that $f^{\prime}$ is non-degenerate.

Lemma 6.2 Suppose $f$ is a non-degenerate signature of arity $n \geq 5$. Let $f^{\prime}$ be $f$ with a self loop. If $f^{\prime} \in \mathscr{P}_{1} \cup \mathscr{M}_{2} \cup \mathscr{A}_{3} \cup \mathscr{M}_{3} \cup \mathscr{V}$ is non-degenerate, then $\mathrm{Pl}-H o l a n t(f)$ is \#P-hard unless $f \in \mathscr{P}_{1} \cup \mathscr{M}_{2} \cup \mathscr{A}_{3} \cup \mathscr{M}_{3} \cup \mathscr{V}$.

Lemma 6.2 depends on several results, each of which handles a different case. In fact, the proof of Lemma 6.2 is a straightforward combination of Corollary 5.5 (for $\mathscr{P}_{1}$ ), Corollary 5.7 (for $\mathscr{A}_{3}$ ), Corollary 5.9 (for $\mathscr{M}_{2} \backslash \mathscr{P}_{2}$ ), and Corollary 5.11 (for $\mathscr{M}_{3}$ ) from Section 5, as well as Corollary 6.4 (for $\mathscr{P}_{2}$ ) and Lemma 6.5 (for $\mathscr{V}$ ), which we will prove shortly. These last two results handle the cases $f^{\prime} \in \mathscr{P}_{2}$ and $f^{\prime} \in \mathscr{V}$ respectively. Note that $\mathscr{M}_{4} \subset \mathscr{V}$. First we consider the case of $f^{\prime} \in \mathscr{P}_{2}$ and show the following lemma.

Lemma 6.3 Let $f$ be a non-degenerate signature of arity $n \geq 5$. If $f=$ $Z^{\otimes n}[a, 1,0, \ldots, 0,1, b]$ for some $a, b \in \mathbb{C}$, where the number of 0 's is $n-3$. Then Pl-Holant $(f)$ is \#P-hard.

Proof First we use the gadget in Fig. 7b, where we put $f$ on both vertices. Let the resulting signature be $h=Z^{\otimes 4} \hat{h}$. It is easier to calculate $\hat{h}$, that is, $h$ in the $Z$ basis. Indeed, $\hat{h}$ is not symmetric, but $\hat{h}$ has the following matrix representation as $n \geq 5$ :

$$
M_{\hat{h}}=\left[\begin{array}{cccc}
0 & a & a & a b+(n-2) \\
a & 2 & 2 & b \\
a & 2 & 2 & b \\
a b+(n-2) & b & b & 0
\end{array}\right] .
$$

Notice that this matrix is redundant, and $\operatorname{det}\left(\widetilde{M}_{\hat{h}}\right)=-4(n-2)(a b+n-2)$. If $a b \neq 2-n$, then by Corollary 2.29 Pl-Holant $(h)$ is \#P-hard, and so is Pl-Holant $(f)$. Hence in the following we assume $a b=2-n$.

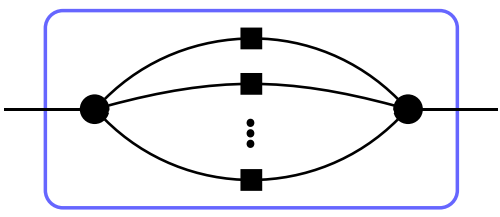

(a)

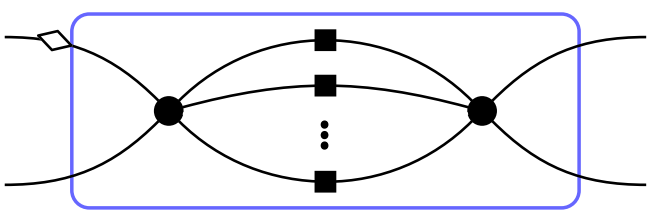

(b)

Fig. 7 Two gadgets used. In the normal basis, circles are assigned $f$ and squares are assigned $=2$. In the $Z$ basis, circles are assigned $\hat{f}$ and squares are assigned $\neq_{2}$. a A binary construction. $\mathbf{b}$ An arity- 4 construction 
Let $f^{\prime}$ be $f$ with a self loop. Recall that $\left(=_{2}\right) Z^{\otimes}=\left(\neq_{2}\right)=[0,1,0]$ up to a nonzero factor. Thus a self loop by $\left(=_{2}\right)$ becomes a self loop by $\left(\neq_{2}\right)$ after the $Z$ transformation. Applying the $Z$ transformation we get the following:

$$
\text { Pl-Holant }\left(=2 \mid f, f^{\prime}\right) \equiv_{T} \text { Pl-Holant }\left([0,1,0] \mid \hat{f}, \widehat{f}^{\prime}\right) \text {, }
$$

where $\widehat{f}^{\prime}=[1,0, \ldots, 0,1]$ and $\hat{f}=[a, 1,0, \ldots, 0,1, b]$ for some $a, b \in \mathbb{C}$. We get this expression of $\widehat{f}^{\prime}$ because doing a self loop commutes with the operation of holographic transformations.

We connect $\widehat{f}^{\prime}$ to $\hat{f}$ via $[0,1,0]$, getting $[a, 2, b]$. Then we connect $[a, 2, b]$ to $\hat{f}$ via $[0,1,0]$ again, getting $\hat{g}=[a b+4, b, 0, \ldots, 0, a, a b+4]$ of arity $n-2$.

If $n \geq 7$, then we use the gadget in Fig. 7b again, where we put $g$ on both vertices this time. We get some signature $h^{\prime}$, which in $Z$ basis has the following matrix representation as $n-2 \geq 5$ :

$$
M_{\widehat{h^{\prime}}}=\left[\begin{array}{cccc}
0 & a(a b+4) & a(a b+4) & (n-4) a b+(a b+4)^{2} \\
a(a b+4) & 2 a b & 2 a b & b(a b+4) \\
a(a b+4) & 2 a b & 2 a b & b(a b+4) \\
(n-4) a b+(a b+4)^{2} & b(a b+4) & b(a b+4) & 0
\end{array}\right] .
$$

Once again this matrix is redundant. It can be simplified as $a b=2-n$. The compressed matrix is

$$
\widetilde{M_{h^{\prime}}}=\left[\begin{array}{ccc}
0 & -2(n-6) a & -6 n+28 \\
-(n-6) a & 8-4 n & -(n-6) b \\
-6 n+28 & -2(n-6) b & 0
\end{array}\right] .
$$

It is easy to compute that $\operatorname{det}\left(\widetilde{M_{\widehat{h^{\prime}}}}\right)=-8(3 n-14)\left(a b(n-6)^{2}-6 n^{2}+40 n-56\right)=$ $8(n-4)(n-2)^{2}(3 n-14)$. Since $n \geq 7, \operatorname{det}\left(\widetilde{M_{h^{\prime}}}\right)>0$. Then by Corollary 2.29 Pl-Holant $\left(h^{\prime}\right)$ is \#P-hard, and so is Pl-Holant $(f)$.

The remaining cases are $n=6$ and $n=5$. When $n=6, a b=2-n=-4$. Moreover, $\hat{g}$ is of arity 4 and $\hat{g}=[a b+4, b, 0, a, a b+4]=[0, b, 0, a, 0]$. We do one more self loop on $g$ via $[0,1,0]$ in the $Z$ basis, resulting in $\widehat{g^{\prime}}=[b, 0, a]$. Connecting $\widehat{g^{\prime}}$ to $\hat{f}$ via $[0,1,0]$, we get $\widehat{g_{1}}=\left[a^{2}, a, 0, b, b^{2}\right]$. Hence $\operatorname{det}\left(\widetilde{M_{\widehat{g_{1}}}}\right)=-4 a^{2} b^{2}=$ $-64 \neq 0$. Then by Corollary 2.29 Pl-Holant $\left(g_{1}\right)$ is \#P-hard, and so is Pl-Holant $(f)$.

At last, $n=5$ and $a b=2-n=-3$. We also have $\hat{g}=[a b+4, b, a, a b+4]=$ $[1, b, a, 1]$. One more self loop on $g$ via $[0,1,0]$ in the $Z$ basis results in $\widehat{g^{\prime \prime}}=[b, a]$. Connecting $\widehat{g^{\prime \prime}}$ to $\hat{f}$ via $[0,1,0]$, we get $\widehat{g_{2}}=\left[a^{2}+b, a, 0, b, b^{2}+a\right]$. Hence $\operatorname{det}\left(\widetilde{M_{\widehat{g_{2}}}}\right)=-2\left(a^{3}+2 a^{2} b^{2}+b^{3}\right)=-2\left(a^{3}+b^{3}+18\right)$. If $a^{3}+b^{3}+18 \neq 0$, then we are done by Corollary 2.29. Otherwise $a^{3}+b^{3}=-18$, and we construct a binary signature $[a, 0, b]$ by doing a self-loop on $\widehat{g_{2}}$ in the $Z$ basis. Then we construct another unary signature by connecting $\widehat{g^{\prime \prime}}=[b, a]$ to $[a, 0, b]$ via $[0,1,0]$, which gives $\left[a^{2}, b^{2}\right]$. Connecting $\left[a^{2}, b^{2}\right]$ to $\hat{f}$ via $[0,1,0]$, we have another arity-4 signature $\widehat{g_{3}}=\left[a b^{2}+a^{2}, b^{2}, 0, a^{2}, a^{2} b+b^{2}\right]$. We compute $\operatorname{det}\left(\widetilde{M_{\widehat{g_{3}}}}\right)=$ $-2\left(a^{6}+a^{5} b^{2}+a^{2} b^{5}+b^{6}\right)=-2\left(a^{6}+b^{6}-162\right)$. If $a^{6}+b^{6}-162 \neq 0$, again we are done by Corollary 2.29. Otherwise $a^{6}+b^{6}=162$. Together with $a^{3}+b^{3}=-18$ and $a b=-3$, there is no solution of $a$ and $b$. This finishes the proof.

This lemma essentially handles the case of $f^{\prime} \in \mathscr{P}_{2}$ due to the following corollary. 
Corollary 6.4 Suppose $f$ is a non-degenerate signature of arity $n \geq 5$. Let $f^{\prime}$ be obtained from $f$ with a self loop. If $f^{\prime} \in \mathscr{P}_{2}$ is non-degenerate, then $\operatorname{Pl-Holant}(f)$ is \#P-hard.

Proof Since $f^{\prime} \in \mathscr{P}_{2}=\mathscr{A}_{2}$, we can assume by Lemma 3.2 that we have the form $c^{-1} f^{\prime}=Z^{\otimes(n-2)}\left(\left[\begin{array}{l}1 \\ 0\end{array}\right]^{\otimes(n-2)}+\beta\left[\begin{array}{l}0 \\ 1\end{array}\right]^{\otimes(n-2)}\right)$, for some $c, \beta \neq$ 0 . Let $\gamma=\beta^{\frac{1}{n-2}}, a=\frac{\gamma+1}{2 \sqrt{\gamma}}$ and $b=\frac{\gamma-1}{2 i \sqrt{\gamma}}$, then $a+b i=\sqrt{\gamma}$ and $a-b i=\frac{1}{\sqrt{\gamma}}$, both nonzero with $a^{2}+b^{2}=1$ and $\frac{a+b i}{a-b i}=\gamma$. Thus $H=\left[\begin{array}{ll}a & b \\ -b & a\end{array}\right] \in \mathbf{O}_{2}(\mathbb{C})$, and $H Z=Z\left[\begin{array}{cc}a+b i & 0 \\ 0 & a-b i\end{array}\right]$. Then $c^{-1} H^{\otimes(n-2)} f^{\prime}=(H Z)^{\otimes(n-2)}\left(\left[\begin{array}{l}1 \\ 0\end{array}\right]^{\otimes(n-2)}+\gamma^{n-2}\left[\begin{array}{l}0 \\ 1\end{array}\right]^{\otimes(n-2)}\right)=(a+$ bi $)^{n-2} Z^{\otimes(n-2)}[1,0, \ldots, 0,1]$ of arity $n-2$. Since $H$ does not change the complexity, we may assume we are under this transformation. Then up to a nonzero constant $f$ is of the form $Z^{\otimes n}[a, 1,0, \ldots, 0,1, b]$ of arity $n$. The claim follows by Lemma 6.3.

The next lemma handles the case when $f^{\prime}$ is a non-degenerate vanishing signature. Its proof is partly contained in the proof of Theorem 64 (p. 1720) in [11]. We include this part here for completeness. As we shall see, the case of $f^{\prime} \in \mathscr{M}_{4}$ is a special case of this result.

Lemma 6.5 Suppose $f$ is a non-degenerate signature of arity $n \geq 5$. Let $f^{\prime}$ be obtained from $f$ with a self loop. If $f^{\prime}$ is non-degenerate and vanishing, then Pl-Holant $(f)$ is \#P-hard unless $\left\{f, f^{\prime}\right\}$ is vanishing, in which case Pl-Holant $(f)$ is tractable.

Proof Since $f^{\prime}$ is vanishing, $f^{\prime} \in \mathscr{V}^{\sigma}$ for some $\sigma \in\{+,-\}$ by Theorem 2.15. For simplicity, assume that $f^{\prime} \in \mathscr{V}^{+}$. The other case is similar.

Note that $f^{\prime}$ is of arity $n-2 \geq 3$. Recall Definition 2.13 and Corollary 2.18. Suppose $\operatorname{rd}^{+}\left(f^{\prime}\right)=d-1$, then $2 d<n$ and $d \geq 2$ since $f^{\prime}$ is non-degenerate. Under the transformation $Z=\frac{1}{\sqrt{2}}\left[\begin{array}{ll}1 & 1 \\ i & -i\end{array}\right]$, we have that

$$
\text { Pl-Holant }\left(=2 \mid f, f^{\prime}\right) \equiv_{T} \text { Pl-Holant }\left([0,1,0] \mid \hat{f}, \hat{f}^{\prime}\right) \text {, }
$$

where $\hat{f}^{\prime}=\left(Z^{-1}\right)^{\otimes n} f^{\prime}=\left[\hat{f}_{1}, \ldots, \hat{f}_{d}, 0, \ldots, 0\right]$ with $\hat{f}_{d} \neq 0$ by Lemma 2.19 . Note that adding a self-loop in the standard basis is the same as connecting to $[0,1,0]$ in the $Z$ basis. Hence we may assume that $\hat{f}=\left[\hat{f}_{0}, \hat{f}_{1}, \ldots, \hat{f}_{d}, 0, \ldots, 0, c\right]$, for some $\hat{f}_{0}$ and $c$. If $c=0$, then $\left\{f, f^{\prime}\right\} \subset \mathscr{V}^{+}$is vanishing. Hence we may assume that $c \neq 0$. We will show that Pl-Holant $(f)$ is \#P-hard.

Doing $d-2$ self-loops by $[0,1,0]$ on $\hat{f}$, we get a signature $\hat{h}=$ $\left[\hat{f}_{d-2}, \hat{f}_{d-1}, \hat{f}_{d}, 0, \ldots, 0,0\right.$ (or) $\left.c\right]$ of arity $n-2(d-2)=n-2 d+4 \geq 5$. The last entry of $\hat{h}$ is $c$ when $d=2$ and is 0 when $d>2$. As $n>2 d$, we may do two more self loops and get $\left[\hat{f}_{d}, 0, \ldots, 0\right]$ of arity $k=n-2 d \geq 1$. This signature is equivalent 
to $[1,0]^{\otimes k}$. Now connect this signature back to $\hat{f}$ via $[0,1,0]$. It is the same as getting the last $n-k+1=2 d+1$ signature entries of $\hat{f}$ up to a nonzero scalar. We may repeat this operation zero or more times until the arity $k^{\prime}$ of the resulting signature is less than or equal to $k$. We claim that this signature has the form $\hat{g}=[0, \ldots, 0, c]$. In other words, the $k^{\prime}+1$ entries of $\hat{g}$ consist of the last $c$ and $k^{\prime}$ many 0's from the signature $\hat{f}$, all appearing after $\hat{f}_{d}$. This is because there are $n-d-1$ many 0 entries in the signature $\hat{f}$ after $\hat{f}_{d}$, and $n-d-1 \geq k \geq k^{\prime}$. Note that $\hat{g}=[0,1]^{\otimes k^{\prime}}$.

Having both $[1,0]^{\otimes k}$ and $\hat{g}=[0,1]^{\otimes k^{\prime}}$ in the $Z$ basis, we realize $[0,1]^{\otimes t}$ using the subtractive Euclidean argument as in Lemma 4.1, where $t=\operatorname{gcd}\left(k, k^{\prime}\right)$. Then we put $\frac{k}{t}$ many copies of $[0,1]^{\otimes t}$ together to get $[0,1]^{\otimes k}$. Connect $\hat{h}$ with $[0,1]^{\otimes k}$ by $[0,1,0]$. Note that due to $[0,1,0]$ flipping the bits, this gets the prefix of $\hat{h}$ of arity $\operatorname{arity}(h)-k$. Recall that $\operatorname{arity}(h)=n-2 d+4$, and hence $\operatorname{arity}(h)-k=$ $n-2 d+4-(n-2 d)=4$. The resulting signature has arity 4 . Moreover, the signature is $\left[\hat{f}_{d-2}, \hat{f}_{d-1}, \hat{f}_{d}, 0,0\right]$. The last entry is 0 (and not $c$ ), because $k \geq 1$ and $\operatorname{arity}(\hat{h}) \geq 5$.

However, as explained in the proof of Lemma 4.7, the problem Pl-Holant $\left([0,1,0] \mid\left[\hat{f}_{d-2}, \hat{f}_{d-1}, \hat{f}_{d}, 0,0\right]\right)$ is equivalent to Pl-Holant $([0,1,0]$ | $[0,0,1,0,0])$ when $\hat{f}_{d} \neq 0$, the problem of counting Eulerian Orientations in planar 4-regular graphs. This problem is \#P-hard [26].

\subsection{Lemmas Applied to Degenerate Signatures in the Inductive Step}

Lemma 6.2 does not solve the case when $f^{\prime}$ is degenerate. In general, when $f^{\prime}$ is degenerate, the inductive step is straightforward unless $f^{\prime}$ is also vanishing. Lemma 6.6 and 6.8 are the two missing pieces to this end.

Lemma 6.6 Let $a, b \in \mathbb{C}$. Suppose $f$ is a signature of the form $Z^{\otimes n}[a, 1,0, \ldots, 0, b]$, where $Z=\left[\begin{array}{ll}1 & 1 \\ i & -i\end{array}\right]$, with arity $n \geq 3$. If $a b \neq 0$, then Pl-Holant $(f)$ is \#P-hard.

Proof We prove by induction on $n$. For $n=3$ or 4 , it follows from Lemma 3.13 and Theorem 2.22 that Pl-Holant $(f)$ is \#P-hard. have

Now assume $n \geq 5$. Under a holographic transformation by $Z=\left[\begin{array}{ll}1 & 1 \\ i & -i\end{array}\right]$, we

$$
\begin{aligned}
\text { Pl-Holant }(=2 \mid f) & \equiv_{T} \text { Pl-Holant }\left([1,0,1] Z^{\otimes 2} \mid\left(Z^{-1}\right)^{\otimes n} f\right) \\
& \equiv_{T} \text { Pl-Holant }([0,1,0] \mid \hat{f}),
\end{aligned}
$$

where $\hat{f}=[a, 1,0, \ldots, 0, b]$. Now consider the gadget in Fig. 7a with $\hat{f}$ assigned to both vertices. This gadget has the binary signature $\widehat{g_{1}}=[0, a b, 2 b]$, which is equivalent to $[0, a, 2]$ since $b \neq 0$. As $\widehat{g_{1}}=\left(Z^{-1}\right)^{\otimes 2} g_{1}$, translating back by $Z$ to the 
original setting $g_{1}=Z^{\otimes 2} \widehat{g_{1}}$, this signature is $g_{1}=[a+1,-i, a-1]$. This can be verified as

$$
\left[\begin{array}{cc}
1 & 1 \\
i & -i
\end{array}\right]\left[\begin{array}{ll}
0 & a \\
a & 2
\end{array}\right]\left[\begin{array}{cc}
1 & 1 \\
i & -i
\end{array}\right]^{\mathrm{T}}=2\left[\begin{array}{cc}
a+1 & -i \\
-i & a-1
\end{array}\right] .
$$

By the form of $\widehat{g_{1}}=[0, a b, 2 b]$ and $b \neq 0$, it follows from Lemma 2.19 that $g_{1} \notin$ $\mathscr{R}_{2}^{+}$. Moreover, since $a \neq 0, g_{1}$ is non-degenerate.

Doing a self loop on $f$ yields $f^{\prime}=Z^{\otimes(n-2)}[1,0, \ldots, 0]$. Connecting $f^{\prime}$ back to $f$, we get a binary signature $g_{2}=Z^{\otimes 2}[0,0, b]$. Once again we connect $g_{2}$ to $f$, the resulting signature is $h=Z^{\otimes(n-2)}[a, 1,0, \ldots, 0]$ of arity $n-2 \geq 3$ up to the constant factor of $b \neq 0$.

Notice that $h$ is non-degenerate and $h \in \mathscr{V}^{+}$. Since $a \neq 0, h \notin \mathscr{M}_{4}^{+}$. By Lemma 4.3, Pl-Holant $\left(h, g_{1}\right)$ is \#P-hard, hence Pl-Holant $(f)$ is also \#P-hard.

The next case uses the following technical lemma. It is also applied more than once in Section 7.

Lemma 6.7 Let $\hat{g}$ be the arity 4 signature whose matrix is

$$
M_{\hat{g}}=\left[\begin{array}{llll}
0 & 0 & 0 & 0 \\
0 & 0 & 1 & 0 \\
0 & 1 & 0 & 0 \\
0 & 0 & 0 & 0
\end{array}\right] .
$$

Then Pl-Holant $\left(\neq_{2} \mid[0,1,0,0,0],[0,0,0,1,0], \hat{g}\right)$ is \#P-hard.

Proof Consider the gadget in Fig. 8a. We assign $[0,0,0,1,0]$ to the triangle vertices, $[0,1,0,0,0]$ to the circle vertices, $\hat{g}$ to the pentagon vertex, and $[0,1,0]$ to the square vertices. Let $\hat{h}$ be the signature of this gadget. By adding two more disequality signatures and then grouping appropriately, it is clear that the gadget in Fig. $8 \mathrm{~b}$ has the same signature of the gadget in Fig. $8 \mathrm{a}$, where the circle vertices are still assigned $[0,1,0,0,0]$, the square vertices are still assigned $[0,1,0]$, and the diamond vertex is assigned the quaternary equality signature. To compute the signature $\hat{h}$, first compute

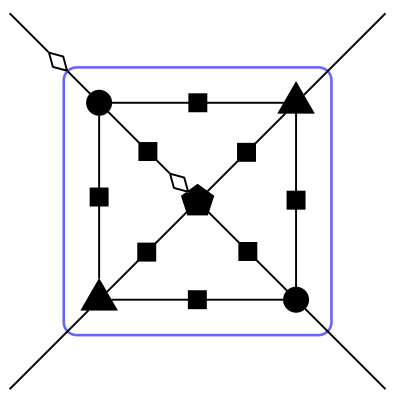

(a)

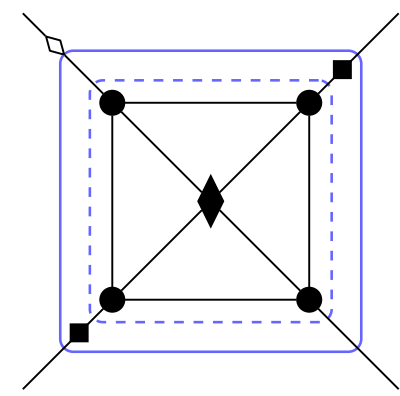

(b)

Fig. 8 Two gadgets with the same signature used in Lemma 6.7. $\mathbf{a}\left(\neq_{2} \mid[0,1,0,0,0],[0,0,0,1,0], \hat{g}\right)$ gate on right side. $\mathbf{b}$ Simpler construction with the same signature 

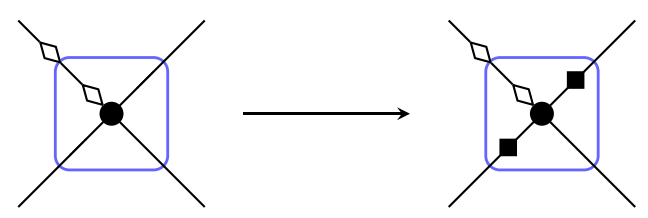

(a)

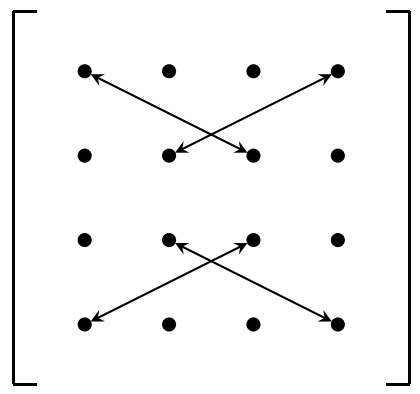

(b)

Fig. 9 The movement of the even Hamming weight entries in the signature matrix of a quaternary signature under the negation of the second and fourth inputs (i.e. the square vertices are assigned [0,1,0]). a Negating the second and fourth inputs. b Movement of even Hamming weight entries

the signature $\hat{h}^{\prime}$ of the inner gadget enclosed by the dashed line, which has signature matrix

$$
M_{\hat{h}^{\prime}}=\left[\begin{array}{llll}
3 & 0 & 0 & 1 \\
0 & 1 & 0 & 0 \\
0 & 0 & 1 & 0 \\
1 & 0 & 0 & 1
\end{array}\right] .
$$

Then by Fig. 9, the signature matrix of $\hat{h}$ is

$$
\hat{h} \text { is } M_{\hat{h}}=\left[\begin{array}{llll}
0 & 0 & 0 & 1 \\
0 & 1 & 3 & 0 \\
0 & 1 & 1 & 0 \\
1 & 0 & 0 & 0
\end{array}\right] .
$$

We use one more gadget before we finish the proof using interpolation. Consider the gadget in Fig. 10b. We assign $\hat{h}$ to the circle vertices and $[0,1,0]$ to the square vertices. The signature of the resulting gadget is $\hat{r}$ with signature matrix $M_{\hat{r}}$ (see

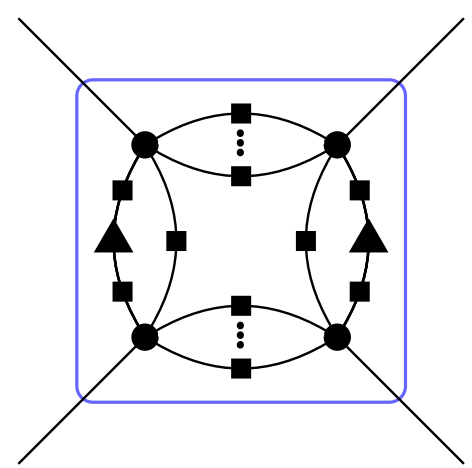

(a)

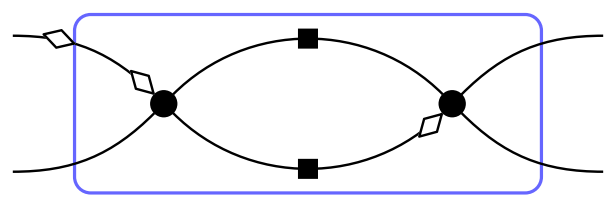

(b)

Fig. 10 Two quaternary gadgets used in the proof of Lemma 6.7 and 6.8. a Gadget that realizes a partial crossover. b Gadget with a useful signature matrix 
Fig. 2 for the signature of a rotated copy of $\hat{h}$ that appears as the second circle vertex in Fig. 10b), where

$$
M_{\hat{r}}=\left[\begin{array}{llll}
0 & 0 & 0 & 1 \\
0 & 1 & 3 & 0 \\
0 & 1 & 1 & 0 \\
1 & 0 & 0 & 0
\end{array}\right]\left(\left[\begin{array}{ll}
0 & 1 \\
1 & 0
\end{array}\right] \otimes\left[\begin{array}{ll}
0 & 1 \\
1 & 0
\end{array}\right]\right)\left[\begin{array}{llll}
0 & 0 & 0 & 1 \\
0 & 1 & 1 & 0 \\
0 & 3 & 1 & 0 \\
1 & 0 & 0 & 0
\end{array}\right]=\left[\begin{array}{llll}
0 & 0 & 0 & 1 \\
0 & 6 & 4 & 0 \\
0 & 4 & 2 & 0 \\
1 & 0 & 0 & 0
\end{array}\right] .
$$

So we can construct $\hat{r}$ in the RHS of Pl-Holant $\left(\neq_{2} \mid[0,1,0,0,0],[0,0,0,1,0], \hat{g}\right)$.

Consider an instance $\Omega$ of Pl-Holant $\left(\neq_{2} \mid \mathcal{F} \cup\left\{\hat{r}^{\prime}\right\}\right)$, where $\mathcal{F}$ is a set of symmetric signatures containing $\hat{r}$, and the signature matrix of $\hat{r}^{\prime}$ is

$$
M_{\hat{r}^{\prime}}=\left[\begin{array}{llll}
0 & 0 & 0 & 1 \\
0 & 3 & 1 & 0 \\
0 & 1 & 1 & 0 \\
1 & 0 & 0 & 0
\end{array}\right]
$$

Suppose that $\hat{r}^{\prime}$ appears $n$ times in $\Omega$. We construct from $\Omega$ a sequence of instances $\Omega_{s}$ of Pl-Holant $\left(\neq_{2} \mid \mathcal{F}\right)$ indexed by $s \geq 1$. We obtain $\Omega_{s}$ from $\Omega$ by replacing each occurrence of $\hat{r}^{\prime}$ with the gadget $N_{s}$ in Fig. 11 with $\hat{r}$ assigned to the circle vertices and $[0,1,0]$ assigned to the square vertices. In $\Omega_{s}$, the edge corresponding to the $i$ th significant index bit of $N_{s}$ connects to the same location as the edge corresponding to the $i$ th significant index bit of $\hat{r}^{\prime}$ in $\Omega$.

We can express the signature matrix of $N_{S}$ as

$$
M_{N_{s}}=X\left(X M_{\hat{r}}\right)^{s}=X P \operatorname{diag}(1,4+2 \sqrt{3}, 4-2 \sqrt{3}, 1)^{s} P^{-1},
$$

where

$$
X=\left[\begin{array}{llll}
0 & 0 & 0 & 1 \\
0 & 0 & 1 & 0 \\
0 & 1 & 0 & 0 \\
1 & 0 & 0 & 0
\end{array}\right] \quad \text { and } \quad P=\left[\begin{array}{cccc}
1 & 0 & 0 & 0 \\
0 & 1 & 1 & 0 \\
0 & \sqrt{3} & -\sqrt{3} & 0 \\
0 & 0 & 0 & 1
\end{array}\right]
$$

Since $M_{\hat{r}^{\prime}}=X P \operatorname{diag}(1,1+\sqrt{3}, 1-\sqrt{3}, 1) P^{-1}$, we can view our construction of $\Omega_{s}$ as first replacing $M_{\hat{r}^{\prime}}$ with $X P \operatorname{diag}(1,1+\sqrt{3}, 1-\sqrt{3}, 1) P^{-1}$, which does not change the Holant value, and then replacing the diagonal matrix with the diagonal matrix $\operatorname{diag}(1,4+2 \sqrt{3}, 4-2 \sqrt{3}, 1)^{s}$.
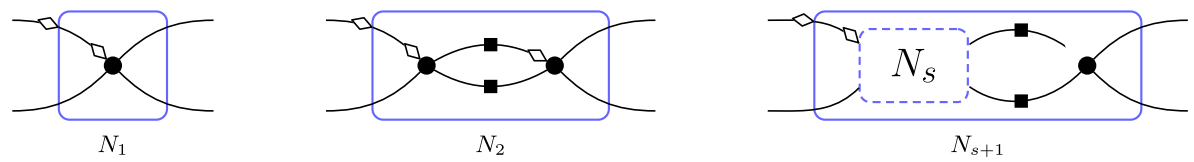

Fig. 11 Linear recursive construction used for interpolation in a nonstandard basis 
We stratify the assignments in $\Omega$ based on the assignments to the $n$ occurrences of the signature whose signature matrix is the diagonal matrix

$$
\left[\begin{array}{cccr}
1 & 0 & 0 & 0 \\
0 & 1+\sqrt{3} & 0 & 0 \\
0 & 0 & 1-\sqrt{3} & 0 \\
0 & 0 & 0 & 1
\end{array}\right] .
$$

We only need to consider the assignments that assign

- $\quad i$ many times the bit patterns 0000 or 1111 ,

- $j$ many times the bit pattern 0110 , and

- $k$ many times the bit pattern 1001 ,

since any other assignment contributes a factor of 0 . Let $c_{i j k}$ be the sum over all such assignments of the products of evaluations of all signatures (including the signatures corresponding to the signature matrices $X, P$, and $P^{-1}$ ) in $\Omega$ except for signature corresponding to the signature matrix in (6.8). Then

$$
\operatorname{Holant}_{\Omega}=\sum_{i+j+k=n}(1+\sqrt{3})^{j}(1-\sqrt{3})^{k} c_{i j k}
$$

and the value of the Holant on $\Omega_{s}$, for $s \geq 1$, is

Holant $_{\Omega_{s}}=\sum_{i+j+k=n}\left((4+2 \sqrt{3})^{j}(4-2 \sqrt{3})^{k}\right)^{s} c_{i j k}=\sum_{i+j+k=n}\left((4+2 \sqrt{3})^{j-k} 4^{k}\right)^{s} c_{i j k}$.

We argue that this Vandermonde system has full rank, which is to say that $(4+2 \sqrt{3})^{j-k} 4^{k} \neq(4+2 \sqrt{3})^{j^{\prime}-k^{\prime}} 4^{k^{\prime}}$ unless $(j, k)=$ $\left(j^{\prime}, k^{\prime}\right)$. If $(4+2 \sqrt{3})^{j-k} 4^{k}=(4+2 \sqrt{3})^{j^{\prime}-k^{\prime}} 4^{k^{\prime}}$, then we have $(4+2 \sqrt{3})^{j-k-\left(j^{\prime}-k^{\prime}\right)} 4^{k-k^{\prime}}=1$. Since any nonzero integer power of $4+2 \sqrt{3}$ is not rational, we must have $j-k=j^{\prime}-k^{\prime}$. And in this case, $4^{k-k^{\prime}}=1$, and hence $k=k^{\prime}$ and $j=j^{\prime}$.

Therefore, we can solve for the unknown $c_{i j k}$ 's and obtain the value of Holant $\Omega$. Thus we have a reduction

$$
\text { Pl-Holant }\left(\neq_{2} \mid \hat{r}^{\prime}\right) \leq_{T} \text { Pl-Holant }\left(\neq_{2} \mid \mathcal{F} \cup\left\{\hat{r}^{\prime}\right\}\right) \leq_{T} \text { Pl-Holant }\left(\neq_{2} \mid \mathcal{F}\right) \text {. }
$$

After a counterclockwise rotation of $\hat{r}^{\prime}$ (c.f. Fig. 2), the rotated form of $\hat{r}^{\prime}$ has matrix

$$
\left[\begin{array}{llll}
0 & 0 & 0 & 3 \\
0 & 1 & 1 & 0 \\
0 & 1 & 1 & 0 \\
1 & 0 & 0 & 0
\end{array}\right],
$$

which is redundant and its compressed form is nonsingular. Hence by Corollary 2.29 the following equivalent problems

$$
\operatorname{Pl-Holant}\left(Z^{\otimes 4} \hat{r}^{\prime}\right) \equiv \operatorname{Pl-Holant}\left(\neq_{2} \mid \hat{r}^{\prime}\right)
$$


are \#P-hard. We conclude that Pl-Holant $\left(\neq_{2} \mid \mathcal{F}\right)$ is \#P-hard. Since we can construct $\hat{r} \in \mathcal{F}$ in the RHS of Pl-Holant $\left(\neq_{2} \mid[0,1,0,0,0],[0,0,0,1,0], \hat{g}\right)$, the lemma follows.

With Lemma 6.7 at hand, we continue to prove Lemma 6.8 .

Lemma 6.8 Let $b \in \mathbb{C}$. Suppose $f$ is a signature of the form $Z^{\otimes n}[0,1,0, \ldots, 0, b]$, where $Z=\left[\begin{array}{ll}1 & 1 \\ i & -i\end{array}\right]$, with arity $n \geq 4$. If $b \neq 0$, then $\mathrm{Pl-Holant}(f)$ is \#P-hard.

Remark 3 For $n=3, Z^{\otimes 3}[0,1,0, b]$ is tractable, as it is $\mathscr{M}$-transformable.

Proof If $n=4$, then we are done by Corollary 2.29. Thus, assume that $n \geq 5$.

Under a holographic transformation by $Z=\left[\begin{array}{ll}1 & 1 \\ i & -i\end{array}\right]$, we have

$$
\begin{aligned}
\operatorname{Pl} \text {-Holant }(=2 \mid f) & \equiv_{T} \text { Pl-Holant }\left([1,0,1] Z^{\otimes 2} \mid\left(Z^{-1}\right)^{\otimes n} f\right) \\
& \equiv_{T} \text { Pl-Holant }([0,1,0] \mid \hat{f}),
\end{aligned}
$$

where $\hat{f}=[0,1,0, \ldots, 0, b]$. We show how to construct the following three signatures: $[0,0,0,1,0],[0,1,0,0,0]$, and $\hat{g}$, where $\hat{g}$ is defined by (6.7). Then we are done by Lemma 6.7.

Consider the gadget in Fig. 7 . We assign $\hat{f}$ to the circle vertices and $[0,1,0]$ to the square vertices. The signature of the resulting gadget is $[0,0,0,1,0]$ up to a nonzero factor of $b$.

Taking a $[0,1,0]$ self loop on $[0,0,0,1,0]$ gives $[0,0,1]=[0,1]^{\otimes 2}$. We connect this back to $\hat{f}$ through $[0,1,0]$ until the arity of the resulting signature is either 4 or 5 , depending on the parity of $n$. If $n$ is even, then we have $[0,1,0,0,0]$ as desired. Otherwise, $n$ is odd and we have $[0,1,0,0,0, b$ or 0$]$, where the last entry is $b$ if $n=5$ and 0 if $n>5$. Connecting $[0,1]^{\otimes 2}$ through $[0,1,0]$ to $\hat{f}$ twice more gives $[0,1]$. We connect this through $[0,1,0]$ to $[0,1,0,0,0, b$ or 0$]$ to get $[0,1,0,0,0]$ as desired.

Taking a $[0,1,0]$ self loop on $[0,1,0,0,0]$ gives $[1,0,0]=[1,0]^{\otimes 2}$. Now consider the gadget in Fig. $7 \mathrm{~b}$. We assign $\hat{f}$ to the circle vertices, $[1,0]^{\otimes 2}$ to the triangle vertices, and $[0,1,0]$ to the square vertices. Up to a factor of $b^{2}$, the signature of the resulting gadget is $\hat{g}$ with signature matrix $M_{\hat{g}}$ given in (6.7). To see this, first replace the two copies of the signatures $[1,0]^{\otimes 2}$ assigned to the triangle vertices with two copies of $[1,0]$ each. Then notice that $\hat{f}$ simplifies to a weighted equality signature when connected to $[1,0]$ through $[0,1,0]$.

\subsection{Proof of the Single Signature Dichotomy}

Now we are ready to prove the dichotomy for a single signature, Theorem 6.1 , which states:

If $f$ is a non-degenerate symmetric signature of arity $n \geq 3$ with complex weights in Boolean variables, then Pl-Holant $(f)$ is \#P-hard unless $f \in \mathscr{P}_{1} \cup$ 
$\mathscr{M}_{2} \cup \mathscr{A}_{3} \cup \mathscr{M}_{3} \cup \mathscr{M}_{4} \cup \mathscr{V}$, in which case the problem is computable in polynomial time.

Recall that $\mathscr{M}_{1} \subset \mathscr{A}_{1} \subset \mathscr{P}_{1}$ and $\mathscr{A}_{2}=\mathscr{P}_{2} \subset \mathscr{M}_{2}$. Thus $f \in \mathscr{P}_{1} \cup \mathscr{M}_{2} \cup \mathscr{A}_{3} \cup \mathscr{M}_{3} \cup$ $\mathscr{M}_{4}$ if and only if $f$ is $\mathscr{A}$-, $\mathscr{P}_{-}$, or $\mathscr{M}$-transformable by Lemma 3.3, Lemma 3.5, or Lemma 3.11.

Proof of Theorem 6.1 The proof is by induction on $n$. The base cases of $n=3$ and $n=4$ are proved in Theorem 2.22. Now assume $n \geq 5$.

With the signature $f$, we form a self loop to get a signature $f^{\prime}$ of arity at least 3 . In general we use prime to denote the signature with a self loop. We consider separately whether or not $f^{\prime}$ is degenerate.

- Suppose $f^{\prime}=[a, b]^{\otimes(n-2)}$ is degenerate. Then there are three cases to consider.

1. If $a=b=0$, then $f^{\prime}$ is the all zero signature. For $f$, this means $f_{k+2}=-f_{k}$ for $0 \leq k \leq n-2$, i.e., $f$ has the form $[x, y,-x,-y, \ldots]$. By Lemma 3.2, $f \in \mathscr{A}_{2}=\mathscr{P}_{2}$, and therefore Pl-Holant $(f)$ is tractable.

2. If $a^{2}+b^{2} \neq 0$, then $f^{\prime}$ is nonzero and $[a, b]$ is not a constant multiple of either $[1, i]$ or $[1,-i]$. We may normalize so that $a^{2}+b^{2}=1$. Then the orthogonal transformation $\left[\begin{array}{ll}a & b \\ -b & a\end{array}\right]$ transforms the column vector $[a, b]$ to $[1,0]$. Let $\hat{f}$ be the transformed signature from $f$, and $\widehat{f}^{\prime}=[1,0]^{\otimes(n-2)}$ the transformed signature from $f^{\prime}$.

Since an orthogonal transformation keeps $=2$ invariant, this transformation commutes with the operation of taking a self loop, i.e., $\widehat{f}^{\prime}=(\hat{f})^{\prime}$. Here $(\hat{f})^{\prime}$ is the function obtained from $\hat{f}$ by taking a self loop. As $(\hat{f})^{\prime}=$ $[1,0]^{\otimes(n-2)}$, we have $\hat{f}_{0}+\hat{f}_{2}=1$ and for every integer $1 \leq k \leq n-2$, we have $\hat{f}_{k}=-\hat{f}_{k+2}$. With one or more self loops on $(\hat{f})^{\prime}$, we eventually obtain either [1,0] when $n$ is odd or $[1,0,0]$ when $n$ is even. In either case, we connect $[1,0]$ or $[1,0,0]$ to $\hat{f}$ until we get an arity 4 signature, which is $\hat{g}=\left[\hat{f}_{0}, \hat{f}_{1}, \hat{f}_{2},-\hat{f}_{1},-\hat{f}_{2}\right]$. This is possible because that the parity matches and the arity of $\hat{f}$ is at least 5 . We show that Pl-Holant $(\hat{g})$ is \#P-hard. To see this, we first compute $\operatorname{det}\left(\widetilde{M}_{g}\right)=-2\left(\hat{f}_{0}+\hat{f}_{2}\right)\left(\hat{f}_{1}^{2}+\hat{f}_{2}^{2}\right)=-2\left(\hat{f}_{1}^{2}+\hat{f}_{2}^{2}\right)$, since $\hat{f}_{0}+\hat{f}_{2}=1$. Therefore if $\hat{f}_{1}^{2}+\hat{f}_{2}^{2} \neq 0$, Pl-Holant $(\hat{g})$ is \#P-hard by Lemma 2.28. Otherwise $\hat{f}_{1}^{2}+\hat{f}_{2}^{2}=0$, and we assume $\hat{f}_{2}=i \hat{f}_{1}$ since the other case $\hat{f}_{2}=-i \hat{f}_{1}$ is similar. Since $f$ is non-degenerate, $\hat{f}$ is nondegenerate, which implies $\hat{f}_{2} \neq 0$. We can rewrite $\hat{g}$ as $[1,0]^{\otimes 4}-\hat{f}_{2}[1, i]^{\otimes 4}$. Under the holographic transformation by $T=\left[\begin{array}{cc}1 & \left(-\hat{f}_{2}\right)^{1 / 4} \\ 0 & i\left(-\hat{f}_{2}\right)^{1 / 4}\end{array}\right]$, we have

$$
\begin{aligned}
& \operatorname{Pl-Holant}(=2 \mid \hat{g}) \equiv_{T} \text { Pl-Holant }\left([1,0,1] T^{\otimes 2} \mid\left(T^{-1}\right)^{\otimes 4} \hat{g}\right) \\
& \equiv_{T} \text { Pl-Holant }(\hat{h} \mid=4) \text {, }
\end{aligned}
$$

where

$$
\hat{h}=[1,0,1] T^{\otimes 2}=\left[1,\left(-\hat{f}_{2}\right)^{1 / 4}, 0\right]
$$


and $\hat{g}$ is transformed by $T^{-1}$ into the arity 4 equality $={ }_{4}$, since

$$
T^{\otimes 4}\left(\left[\begin{array}{l}
1 \\
0
\end{array}\right]^{\otimes 4}+\left[\begin{array}{l}
0 \\
1
\end{array}\right]^{\otimes 4}\right)=\left[\begin{array}{l}
1 \\
0
\end{array}\right]^{\otimes 4}-\hat{f_{2}}\left[\begin{array}{l}
1 \\
i
\end{array}\right]^{\otimes 4}=\hat{g} .
$$

By Theorem 2.24, Pl-Holant $(\hat{h} \mid=4)$ is \#P-hard as $\hat{f}_{2} \neq 0$.

3. If $a^{2}+b^{2}=0$ but $(a, b) \neq(0,0)$, then $[a, b]$ is a nonzero multiple of $[1, \pm i]$. Ignoring the constant multiple, we have $f^{\prime}=[1, i]^{\otimes(n-2)}$ or $[1,-i]^{\otimes(n-2)}$. We consider the first case since the other case is similar.

In the first case, the characteristic polynomial of the recurrence relation of $f^{\prime}$ is $x-i$, so that of $f$ is $(x-i)\left(x^{2}+1\right)=(x-i)^{2}(x+i)$. Hence there exist $a_{0}, a_{1}$, and $c$ such that

$$
f_{k}=\left(a_{0}+a_{1} k\right) i^{k}+c(-i)^{k}
$$

for every integer $0 \leq k \leq n$. Let $f^{+}$and $f^{-}$be two signatures of arity $n$ such that $f_{k}^{+}=\left(a_{0}+\bar{a}_{1} k\right) i^{k}$ and $f_{k}^{-}=c(-i)^{k}$ for every $0 \leq k \leq n$. Hence $f_{k}=f_{k}^{+}+f_{k}^{-}$and we write $f=f^{+}+f^{-}$. If $a_{1}=0$, then $f^{\prime}$ is the all zero signature, a contradiction. If $c=0$, then $f$ is vanishing, one of the tractable cases. Now we assume $a_{1} c \neq 0$ and show that Pl-Holant $(f)$ is \#P-hard. Hence $\mathrm{rd}^{+}\left(f^{+}\right)=1$ and $\mathrm{rd}^{-}\left(f^{-}\right)=0$. Thus, $\left(Z^{-1}\right)^{\otimes n}\left(f^{+}\right)$ has the form $[x, y, 0, \ldots, 0]$, where $y \neq 0$, and $\left(Z^{-1}\right)^{\otimes n}\left(f^{-}\right)$has the form $[0,0, \ldots, 0, z]$, where $z=2^{n / 2} c \neq 0$. It follows that $\hat{f}=\left(Z^{-1}\right)^{\otimes n}(f)=$ $[x, y, 0, \ldots, 0, z]$. Note that the arity $n \geq 5$, so the nonzero entries in the sum do not collide.

Under the holographic transformation $Z=\frac{1}{\sqrt{2}}\left[\begin{array}{ll}1 & 1 \\ i & -i\end{array}\right]$, we have

$$
\begin{aligned}
\text { Pl-Holant }(=2 \mid f) & \equiv_{T} \text { Pl-Holant }\left([1,0,1] Z^{\otimes 2} \mid\left(Z^{-1}\right)^{\otimes n} f\right) \\
& \equiv_{T} \text { Pl-Holant }([0,1,0] \mid \hat{f}) \\
& \equiv_{T} \text { Pl-Holant }\left(Z^{\otimes n} \hat{f}\right),
\end{aligned}
$$

where $\hat{f}$ takes the form $\left[\hat{f}_{0}, \hat{f}_{1}, 0, \ldots, 0, \hat{f}_{n}\right]$ with $\hat{f}_{n}=2^{n / 2} c \neq 0$ and $\hat{f}_{1} \neq$ 0 . On the other side, $\left(=_{2}\right)=[1,0,1]$ is transformed into $\left(\neq_{2}\right)=[0,1,0]$. Depending on whether $\hat{f}_{0}=0$ or not, we apply Lemma 6.8 or Lemma 6.6 and Pl-Holant $(f)$ is \#P-hard.

- Suppose $f^{\prime}$ is non-degenerate. By the inductive hypothesis, Pl-Holant $\left(f^{\prime}\right)$ is \#Phard, unless $f^{\prime} \in \mathscr{P}_{1} \cup \mathscr{M}_{2} \cup \mathscr{A}_{3} \cup \mathscr{M}_{3} \cup \mathscr{M}_{4} \cup \mathscr{V}$. Note that $f^{\prime}$ has arity $n-2 \geq 3$, and every signature in $\mathscr{M}_{4}$ of arity at least 3 is also in $\mathscr{V}$. Hence the exceptional case is equivalent to $f^{\prime} \in \mathscr{P}_{1} \cup \mathscr{M}_{2} \cup \mathscr{A}_{3} \cup \mathscr{M}_{3} \cup \mathscr{V}$. In this case, we apply Lemma 6.2 to $f^{\prime}$ and $f$. Hence Pl-Holant $(f)$ is \#P-hard, unless $f \in \mathscr{P}_{1} \cup \mathscr{M}_{2} \cup \mathscr{A}_{3} \cup \mathscr{M}_{3} \cup \mathscr{V}$. The exceptional cases imply that $f$ is $\mathscr{A}$ - or $\mathscr{P}$ or $\mathscr{M}$-transformable or vanishing, and Pl-Holant $(f)$ is tractable. 


\section{Mixing $\mathscr{P}_{2}$ and $\mathscr{M}_{4}$-Equalities and Matchgates in the $Z$ Basis}

Given a set $\mathcal{F}$ of symmetric signatures, by Theorem $6.1, \operatorname{Pl}-H o l a n t(\mathcal{F})$ is \#P-hard unless every single non-degenerate signature $f$ of arity at least 3 in $\mathcal{F}$ is in $\mathscr{P}_{1} \cup$ $\mathscr{M}_{2} \cup \mathscr{A}_{3} \cup \mathscr{M}_{3} \cup \mathscr{M}_{4} \cup \mathscr{V}$. Furthermore, we have already proved that the desired full dichotomy holds if $\mathcal{F}$ contains such an $f$ in $\mathscr{P}_{1}, \mathscr{A}_{3}, \mathscr{M}_{2} \backslash \mathscr{P}_{2}$, or $\mathscr{M}_{3}$ due to Corollary 5.4, Corollary 5.6, Lemma 5.8, or Lemma 5.10, respectively, namely, if $\mathcal{F}$ contains a non-degenerate signature $f \in \mathscr{P}_{1} \cup\left(\mathscr{M}_{2} \backslash \mathscr{P}_{2}\right) \cup \mathscr{A}_{3} \cup \mathscr{M}_{3}$ of arity at least 3 , then Pl-Holant $(\mathcal{F})$ is \#P-hard unless $\mathcal{F}$ is $\mathscr{A}-$, $\mathscr{P}$-, or $\mathscr{M}$-transformable, in which case Pl-Holant $(\mathcal{F})$ is tractable.

The remaining cases are when all non-degenerate signatures of arity at least 3 in $\mathcal{F}$ are contained in $\mathscr{P}_{2} \cup \mathscr{M}_{4} \cup \mathscr{V}$. In this section, we consider the mixing of $\mathscr{P}_{2}$ and $\mathscr{M}_{4}$. For this, we do a holographic transformation by $Z=\left[\begin{array}{ll}1 & 1 \\ i & -i\end{array}\right]$ or $Z\left[\begin{array}{ll}0 & 1 \\ 1 & 0\end{array}\right]=$ $\left[\begin{array}{ll}1 & 1 \\ -i & i\end{array}\right]$. Then the problem becomes Pl-Holant $\left(\neq_{2} \mid=_{k}, \operatorname{EXACTONE}_{d}\right)$ with various arities $k$ and $d$. Recall that EXACTONE $_{d}$ denotes the exact one function $[0,1,0, \ldots, 0]$ of arity $d$. These are the signatures for PERFECT MATCHING and they are the basic components of Matchgates.

At this stage of our proof, we found a big surprise. Against the putative form of a complexity classification for planar counting problems, we found that the complexity of the problem Pl-Holant $\left(\neq_{2} \mid={ }_{k}\right.$, EXACTONE $\left.d\right)$ depends on the values of $d$ and $k$, and the problem is tractable for all large $k$. This result has the consequence that, for the first time since Kasteleyn's algorithm, we have discovered some new primitive tractable family of counting problems on planar graphs. These problems cannot be captured by a holographic reduction to Kasteleyn's algorithm, or any other known algorithm. Thus for planar problems the paradigm of holographic algorithms using matchgates (i.e., being $\mathscr{M}$-transformable) is not universal.

Let $\mathcal{E O}=\left\{\right.$ EXACTONE $\left._{d} \mid d \geq 3\right\}$.

\subsection{Hardness when $k=3$ or 4}

We begin with some hardness results.

Lemma 7.1 Pl-Holant $\left(\neq_{2} \mid={ }_{3},[0,1,0,0]\right)$ is \#P-hard.

Proof By connecting two copies of $[0,1,0,0]$ together via $\neq_{2}$, we have $[0,1,0,0,0]$ on the right. Consider the gadget in Fig. 12a. We assign $=3$ to the triangle vertices, $[0,1,0,0]$ to the circle vertices, $\neq_{2}$ to the square vertices, and $[0,1,0,0,0]$ on the diamond vertex in the middle. Let $f$ be the signature of this gadget.

We claim that the support of $f$ is $\{0011,0110,1100,1001\}$. To see this, notice that $[0,1,0,0,0]$ in the middle must match exactly one of the half edges, which forces the corresponding equality signature to take the value 0 and all other equality signatures to take value 1 . The two $[0,1,0,0]$ 's adjacent to the equality assigned 0 must have 0 going out, and the other two $[0,1,0,0]$ 's have 1 going out. 


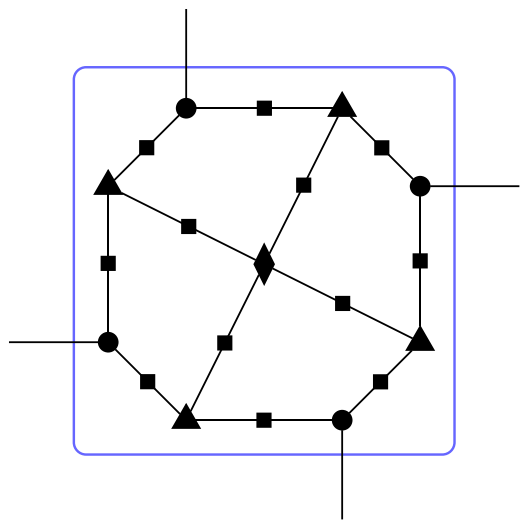

(a)

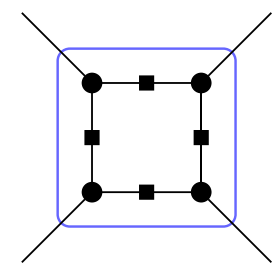

(b)

Fig. 12 Two gadgets used in the proof of Lemma 7.1. a Cycle-like gadget used twice. b Gadget to realize $\hat{g}$

Now we consider the gadget in Fig. 12a again. This time we place $[0,1,0,0]$ on each triangle, $=3$ on each circle, $f$ on the middle diamond, and again $\neq 2$ on each square. Now notice that each support of $f$ makes two $[0,1,0,0]$ 's that are cyclically adjacent on the outer cycle to become $[0,1,0]$ and the other two $[1,0,0]$. It is easy to see that the support of the resulting signature is $\{0111,1011,1101,1110\}$. Therefore it is the reversed ExACTONE 4 signature $[0,0,0,1,0]$ (namely ALLBUTONE 4 ). The whole gadget is illustrated in Fig. 13, where each circle is assigned $[0,1,0,0]$, triangle $=_{3}$, and square $\neq_{2}$.

Finally, we build the gadget in Fig. 12b. We place $=_{3}$ on each circle and $\neq_{2}$ on each square. It is easy to see that there are only two support vectors of the result-

Fig. 13 The whole gadget to realize $[0,0,0,1,0]$

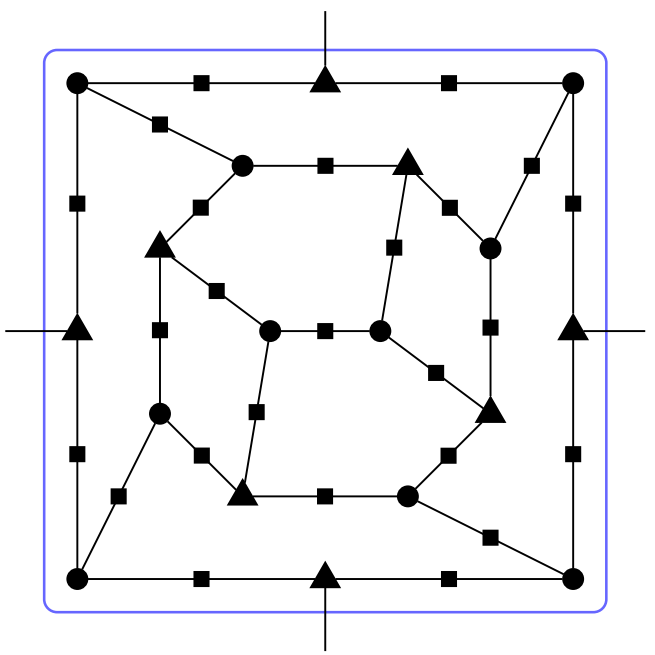


ing signature, which are 0101 and 1010. Recall the definition (6.7) of the partial crossover $\hat{g}$. This gadget realizes exactly $\hat{g}$.

By Lemma 6.7, Pl-Holant $\left(\neq_{2} \mid[0,1,0,0,0],[0,0,0,1,0], \hat{g}\right)$ is \#P-hard. We have constructed $[0,1,0,0,0],[0,0,0,1,0]$, and $\hat{g}$ on the right side. Therefore Pl-Holant $\left(\neq_{2} \mid=3,[0,1,0,0]\right)$ is \#P-hard.

For $k=4$, we need the following lemma.

Lemma 7.2 Let $g$ be the arity 4 signature whose matrix is

$$
M_{g}=\left[\begin{array}{llll}
2 & 0 & 0 & 0 \\
0 & 1 & 0 & 0 \\
0 & 0 & 1 & 0 \\
0 & 0 & 0 & 1
\end{array}\right] .
$$

Then Pl-Holant $(g)$ is \#P-hard.

Proof Let $h=[2,1,1]$. We show that Pl-\#CSP $(h) \leq_{T}$ Pl-Holant $(g)$ in two steps. In each step, we begin with a signature grid and end with a new signature grid such that the Holant values of both signature grids are the same. This follows from Theorem 2.24, since Pl-\#CSP $(h) \equiv \operatorname{Pl-Holant~}(\mathcal{E} \mathcal{Q} \mid h)$ by $(2.2)$.

For step one, let $G=(U, V, E)$ be an instance of Pl-Holant $(\mathcal{E} \mathcal{Q} \mid h)$. Fix an embedding of $G$ in the plane. This defines a cyclic ordering of the edges incident to each vertex. Consider a vertex $u \in U$ of degree $k$. It is assigned the signature $=_{k}$. We decompose $u$ into $k$ vertices. Then we connect the $k$ edges originally incident to $u$ to these $k$ new vertices so that each vertex is incident to exactly one edge. We also connect these $k$ new vertices in a cycle according to the cyclic ordering induced on them by their incident edges. Each of these vertices has degree 3, and we assign them $={ }_{3}$. Clearly the Holant value is unchanged. This completes step one. An example of this step applied to a vertex of degree 4 is given in Fig. 14a. The resulting graph has the following properties: (1) it is planar; (2) every vertex is either degree 2 (in $V$ and assigned $h$ ) or degree 3 (newly created and assigned $={ }_{3}$ ); (3) each degree 2 vertex is connected to two degree 3 vertices; and (4) each degree 3 vertex is connected to one degree 2 vertex and two other degree 3 vertices.

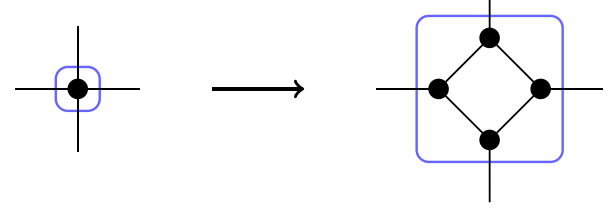

(a)

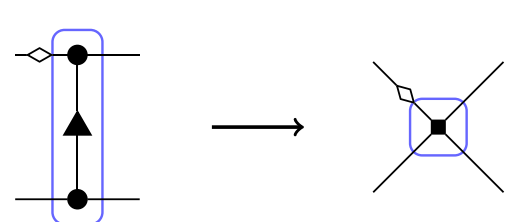

(b)

Fig. 14 A reduction from Pl-Holant $(\mathcal{E} \mathcal{Q} \mid h)$ to Pl-Holant $(g)$ for any binary signature $h$ and a quaternary signature $g$ that depends on $h$. The circle vertices are assigned $=_{4}$ or $=3$ respectively, the triangle vertex is assigned $h$, and the square vertex is assigned the signature of the gadget to its left. a Step one: Degree 4 vertex example. b Step two: Contract edges 
Now step two. For every $v \in V, v$ has degree 2 . We contract the two edges incident to $v$, or equivalently, we replace the two circle vertices and one triangle vertex boxed in Fig. 14b with a single (square) vertex of degree 4 . The resulting graph $G^{\prime}=$ $\left(V^{\prime}, E^{\prime}\right)$ is planar and 4-regular.

Next we determine what is the signature on $v^{\prime} \in V^{\prime}$ after this contraction. Clearly the two inputs to each original circle have to be the same (as illustrated in the first figure in Fig. 14b). Therefore its support is 0000,0110,1001,1111, listed starting from the diamond and going counterclockwise. Moreover, due to the triangle assigned $h$ in the middle, the weight on 0000 is 2 , and every other weight is 1 . Hence it is exactly the signature $g$, with the diamond in Fig. 14b marking the first input bit. This finishes the proof.

Remark 4 From the planar embedding of the graph $G$, treating $h$ vertices as edges, the resulting graph $G^{\prime}$ is known as the medial graph of $G$. The (constructive) definition is usually phrased in the following way. The medial graph $G_{m}$ of plane graph $G$ has a vertex on each edge of $G$ and two vertices in $G_{m}$ are joined by an edge for each face of $G$ in which their corresponding edges occur consecutively. See Fig. 15 for an example. However, our construction described in the proof clearly extends to nonplanar graphs as well.

Lemma 7.3 Pl-Holant $\left(\neq_{2} \mid={ }_{4},[0,1,0,0]\right)$ is \#P-hard.

Proof Consider the gadget in Fig. 16. We assign binary disequality $\neq_{2}$ to the square vertices, $={ }_{4}$ to the circle vertices, and $[0,1,0,0]$ to the triangle vertices. We show that the support of the resulting signature is the set $\{00110011,11001100,11111111\}$, where each vector is the assignment ordered counterclockwise starting from the diamond point.

We call the equality signature $=_{4}$ in the middle the origin. There are two possible assignments at the origin. If it is assigned 0 , then every adjacent perfect matching

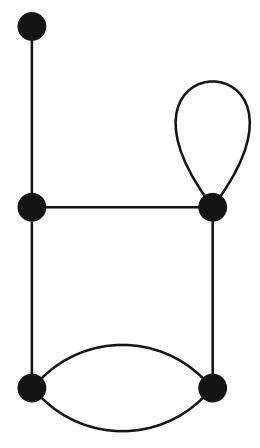

(a)

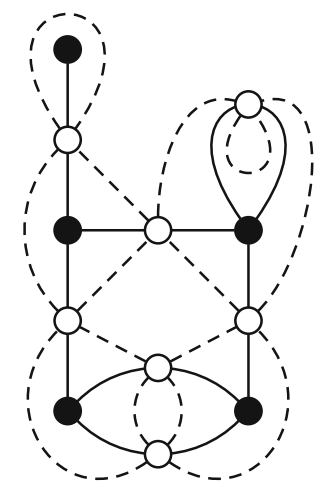

(b)

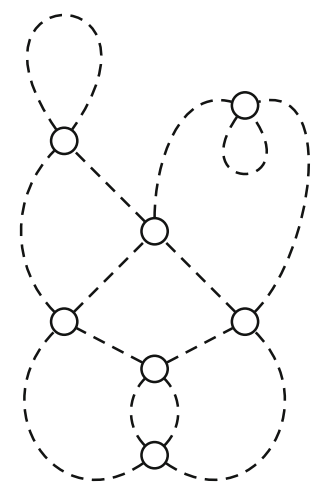

(c)

Fig. 15 A plane graph (a), its medial graph (c), and both graphs superimposed (b) 
Fig. 16 Grid-like gadget used in the proof of Lemma 7.3, whose support vectors are 00110011, 11001100, and 11111111. Each square is assigned a binary disequality $\neq_{2}$, circle $=_{4}$, and triangle $[0,1,0,0]$

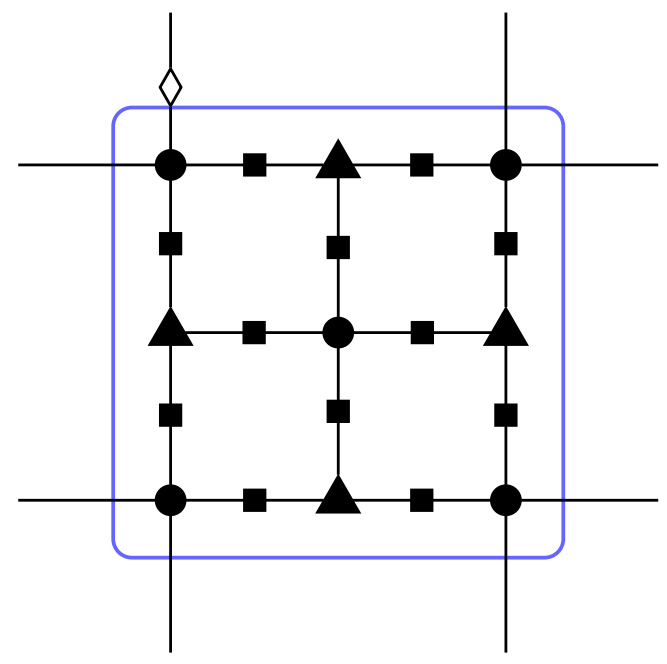

signature $[0,1,0,0]$ is matched to the half edge towards the origin, and every equality $=4$ is forced to be 1 . This gives the support vector 11111111 .

The other possibility is that the origin is 1 . In this case, we can remove the origin leaving the outer cycle, with every $[0,1,0,0]$ becoming $[0,1,0]$. This is effectively a cycle of four equalities connected by $\neq_{2}$. It is easy to see that there are only two support vectors, which are exactly 00110011 and 11001100.

Every pair of half edges at each corner always take the same value. We further connect each pair of these edges to different copy of $=_{4}$ via two copies of $\neq_{2}$. This results in a gadget with signature $f$ whose support is the complement of the original support, that is, $\{11001100,00110011,00000000\}$.

Now consider the gadget in Fig. $17 \mathrm{a}$. We assign $\neq_{2}$ to the square vertices, $=4$ to the circle vertices, $[0,1,0,0]$ to the triangle vertices, and $f$ to the pentagon vertex. Notice that each pair of edges coming out of the pentagon vertex are from the same corner of the gadget in Fig. 16 used to realize $f$. We now study the signature of this gadget.

Notice that if an $=_{4}$ on the outer cycle is assigned 0 , then the two adjacent perfect matchings must match half edges toward that $=4$, and their outgoing edges must be 0 . Furthermore, the two $=_{4}$ one more step away must be 1 . A further observation is that any pair of consecutive $=4$ 's cannot be both 0 , and if a pair of consecutive $=4$ 's are both 1 , then the $[0,1,0,0]$ between them must have a 1 going out. In Fig. 17a, we call the pentagon connecting to four equalities $={ }_{4}$ on the upper right $f_{1}$ and the other one $f_{2}$. Let $g$ be the signature of resulting gadget. We further order the external wires of $f_{1}, f_{2}$, and $g$ counterclockwise, each starting from edge marked with a diamond. With this notation and these observations, we get Table $17 \mathrm{~b}$ listing the support of $g$. The support of $g$ is $\{11111111,01111000,11110000,10000111,00000000,00001111,00000000\}$, and 00000000 has multiplicity 2.

Next we use a domain pairing argument. First we move $=_{4}$ to the left hand side, by connecting four $\neq_{2}$ into it. We apply the domain pairing on the problem 


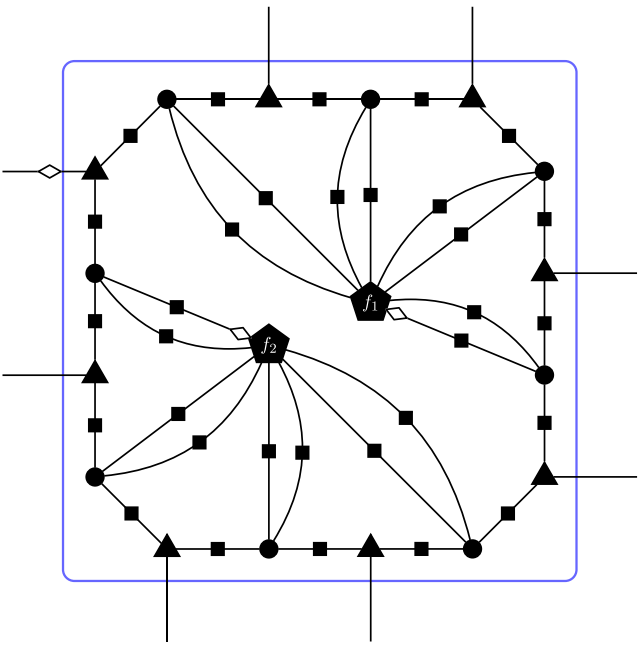

(a)

\begin{tabular}{|c|c||c|}
\hline$f_{1}$ & $f_{2}$ & $g$ \\
\hline \hline 00000000 & 00000000 & 11111111 \\
\hline 00110011 & 00000000 & 01111000 \\
\hline 11001100 & 00000000 & 11110000 \\
\hline 00000000 & 00110011 & 10000111 \\
\hline 00110011 & 00110011 & 00000000 \\
\hline 11001100 & 00110011 & - \\
\hline 00000000 & 11001100 & 00001111 \\
\hline 00110011 & 11001100 & - \\
\hline 11001100 & 11001100 & 00000000 \\
\hline
\end{tabular}

(b)

Fig. 17 Another gadget used in the proof of Lemma 7.3 and a Table listing the support of its signature. a Gadget with signature $g$. Each square is assigned a binary disequality $\neq 2$, circle $=4$, triangle $[0,1,0,0]$, and pentagon $f$. b Support of $g$. Each vector is an assignment ordered counterclockwise from the diamond.

Pl-Holant $\left(=_{4} \mid g\right)$. Specifically, we use $=_{4}$ as $=_{2}$, by pairing each pair of edges together. We also pair adjacent two outputs of $g$ counterclockwise, starting from the diamond point. Each pair of output wires of $g$ are connected to a pair of wires from $={ }_{4}$ on the left hand side. Note that $={ }_{4}$ enforces that each pair of edges always takes the same value. We re-interpret 00 or 11 as 0 or 1 in the Boolean domain. In this way, we can treat $g$ as an arity 4 signature $g^{\prime}$ in the Boolean domain. So the reduction is

$$
\text { Pl-Holant }\left(=2 \mid g^{\prime}\right) \leq_{T} \text { Pl-Holant }\left(=_{4} \mid g\right) \text {. }
$$

We get the expression of $g^{\prime}$ next. The two support bit strings 01111000 and 10000111 of $g$ are eliminated as they do not agree on adjacent paired outputs. So in the paired (Boolean) domain, the support of $g^{\prime}$ becomes $\{1111,1100,0011,0000\}$ where 0000 has multiplicity 2. We further rotate $g^{\prime}$ as a Boolean domain signature such that the support is $\{1111,0110,1001,0000\}$. Now it is easy to see that the matrix of $g^{\prime}$, an arity 4 signature in the Boolean domain, is

$$
M_{g^{\prime}}=\left[\begin{array}{llll}
2 & 0 & 0 & 0 \\
0 & 1 & 0 & 0 \\
0 & 0 & 1 & 0 \\
0 & 0 & 0 & 1
\end{array}\right] .
$$

By Lemma 7.2 Pl-Holant $\left(g^{\prime}\right)$ is \#P-hard. Hence Pl-Holant $\left(\neq_{2} \mid=_{4},[0,1,0,0]\right)$ is \#P-hard.

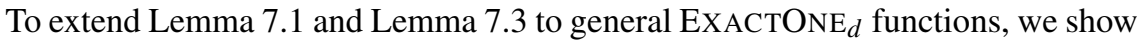
that we can always realize constant functions $[1,0]$ and $[0,1]$ in this setting. 
Lemma 7.4 For any integer $k \geq 3$ and $d \geq 3$ and any signature set $\mathcal{F}$ consisting of nonnegative integer valued signatures,

$\operatorname{Pl}$-Holant $\left(\neq_{2} \mid={ }_{k}\right.$, EXACTONE $\left._{d},[0,1], \mathcal{F}\right) \leq_{T}$ Pl-Holant $\left(\neq_{2} \mid={ }_{k}\right.$, ExACTONE $\left._{d}, \mathcal{F}\right)$.

Furthermore, we can construct $=_{\ell k}$ and $\mathrm{EXACTONE}_{2+\ell(d-2)}$ in the right hand side, for all $\ell \geq 1$.

Proof We can construct $=\ell k$ on the right, for any integer $\ell \geq 1$, by $\neq 2$ on the left and $={ }_{k}$ on the right, as follows. If we connect two copies of $=_{k}$ via $\neq 2$ we get a signature of arity $2 k-2$ with $k-1$ consecutive external wires labeled + and the others labeled -. As $k \geq 3$, we can take 2 wires of the $k-1$ wires labeled - and attach to two copies of $=_{k}$ via two $\neq_{2}$. This creates a signature of arity $3(k-1)+(k-3)$ with $3(k-1)$ consecutive wires labeled + and the other $k-3$ wires labeled - . Finally connect $k-3$ pairs of adjacent $+/-$ labeled wires by $\neq 2$ recursively. This creates a planar gadget with an equality signature of arity $3(k-1)-(k-3)=2 k$, up to a nonzero constant factor. This can be extended to any $=\ell k$ by applying the same process on any consecutive $k$ wires.

We construct $\mathrm{EXACTONE}_{2+\ell(d-2)}$ on the right, for any integer $\ell \geq 1$, by connecting $\ell$ many copies of EXACTONE $E_{d}$ sequentially via $\neq \neq_{2}$.

Next we construct $[0,1]^{\otimes r}$ for some integer $r \geq 1$ on the right hand side. We get $[1,0]^{\otimes(d-2)}$ by a self-loop of EXACTONE $d$ via $\neq 2$, ignoring the factor 2 . We pick an integer $\ell$ large enough so that $d-2<\ell k$. Then we connect $[1,0]^{\otimes(d-2)}$ to $=\ell k$ via $\neq 2$ to get $[0,1]^{\otimes(\ell k-d+2)}$. Thus $r=\ell k-d+2$ suffices.

Given an instance $\Omega$ of Pl-Holant $\left(\neq_{2} \mid=_{k}\right.$, ExACTONE $\left._{d},[0,1], \mathcal{F}\right)$ with an underlying plane graph $G$, consider the dual graph $G^{*}$ of $G$. Take a spanning tree $T$ of $G^{*}$, with the external face as the root. In each face $F$, let $c_{F}$ be the number of $[0,1]$ 's in the face $F$. We start from the leaves to recursively move all the pinnings of $[0,1]$ to the external face. Suppose we are working on the face $F$ as a leaf of $T$, and it is not the root (the external face). If $c_{F}=0$ then we just remove the leaf from $T$ and recurse on another leaf. Otherwise we remove all $[0,1]$ 's in $F$, creating $c_{F}$ dangling edges in the face $F$. Let $s$ be the smallest integer such that $s r \geq c_{F}$. We replace the $\neq_{2}$ edge bordering between $F$ and its parent $F^{\prime}$ by a chain of three signatures: $\neq_{2}$, $\operatorname{EXACTONE}_{2+\ell(d-2)}$ and $\neq_{2}$, where $\ell$ is an integer such that $\ell(d-2) \geq s r-c_{F}$. (Notice that if $\ell(d-2)$ many dangling edges of $\mathrm{EXACTONE}_{2}+\ell(d-2)$ are all pinned to 0 , then this chain becomes a chain of three $\left(\neq_{3}\right)$ 's, and thus functionally equivalent to the original single $\neq_{2}$ between $F$ and $F^{\prime}$.) From EXACTONE $2+\ell(d-2)$ there are two edges connected to the two adjacent copies of $\neq_{2}$. Of the other $\ell(d-2)$ edges we will put $s r-c_{F}$ many dangling edges in $F$, and the remaining $\ell(d-2)-\left(s r-c_{F}\right)$ dangling edges in $F^{\prime}$. Hence there are $s r$ dangling edges in $F$, including those $c_{F}$ many that were connected to $[0,1]$ 's via $\neq_{2}$ before we removed the $[0,1]$ 's. We put $s$ copies of $[0,1]^{\otimes r}$ inside the face $F$ to pin all of them in a planar way. We add $\ell(d-2)-\left(s r-c_{F}\right)$ to $c_{F^{\prime}}$, and they are all pinned to 0 (by connecting to $[0,1]$ via $\neq_{2}$ ). Remove the leaf $F$ from $T$, and recurse.

After the process, all $[0,1]$ 's are in the external face of $G$. Suppose the number is $p$. We put $r$ disjoint copies of $G$ together to form a planar signature grid. Apply a total of $p r$ many [0,1]'s by $p$ copies of $[0,1]^{\otimes r}$ in a planar way. This is now an instance of 
$\operatorname{Pl}$-Holant $\left(\neq_{2} \mid=_{k}\right.$, EXACTONE $\left._{d}, \mathcal{F}\right)$ and the Holant value is the $r$ th power of that of $\Omega$. Since the Holant value of $\Omega$ is a nonnegative integer, we can take the $r$ th root and finish the reduction.

Once we have the pinning $[0,1]$ in the right hand side of Pl-Holant $\left(\neq_{2} \mid=_{k}\right.$, EXACTONE $\left._{d}\right)$, it is easy to construct EXACTONE $3=[0,1,0,0]$ from EXACTONE $_{d}$, for all $d \geq 3$, because connecting $[0,1]$ via $\neq_{2}$ to EXACTONE $_{d}$ gives EXACTONE $_{d-1}$. Therefore combining Lemma 7.4 with Lemma 7.1 and Lemma 7.3 we get the following corollary.

Corollary 7.5 If $d \geq 3$ and $k \in\{3,4\}$, then Pl-Holant $\left(\neq_{2} \mid={ }_{k}\right.$, EXACTONE $\left._{d}\right)$ is \#P-hard.

\subsection{Tractability when $k \geq 5$}

On the other hand, if the arity $k$ of the equality signature is at least 5 , then $\operatorname{Pl}$-Holant $\left(\neq_{2} \mid=_{k}, \mathcal{E O}\right)$ is tractable, where $\mathcal{E O}=\left\{\right.$ EXACTONE $\left._{d} \mid d \geq 3\right\}$. In this subsection we will first prove that this problem is tractable for $k \geq 6$. After that we will return to $=5$.

To prove this, we first do some preprocessing. Let $G$ be the underlying graph of an instance of Pl-Holant $\left(\neq_{2} \mid==_{k}, \mathcal{E O}\right)$. First, we may assume no copy of $=_{k}$ has a self loop by $\neq_{2}$, since this results in 0 for the overall Holant value. Similarly we may assume that no EXACTONE $d$ in $\mathcal{E O}$ has more than one self loops by $\neq_{2}$, since this also results in 0 . If a single self loop by a $\neq_{2}$ is applied to some EXACTONE Ex $_{d}$, the signature EXACTONE $d$ is changed to $[1,0]^{\otimes(d-2)}$ with a factor 2 . In this way we can eliminate all self loops by $\neq_{2}$ on all signatures on the RHS, resulting in some pinning signatures $[1,0]$. These pinning signatures can be applied recursively. Any $[1,0]$ is first transformed to $[0,1]$ via $\neq_{2}$ on LHS and then applied either to $=_{k}$ producing $[0,1]^{\otimes(k-1)}$, or to EXACTONE $_{d}$ (for some $d$ ) producing $[1,0]^{\otimes(d-1)}$. Similarly, any $[0,1]$ is first transformed to $[1,0]$ via $\neq_{2}$ on LHS and then applied either to $=_{k}$ producing $[1,0]^{\otimes(k-1)}$, or to EXACTONE $_{d}$ (for some $d$ ) producing EXACTONE $_{d-1}$. (Of course if a pair of pinning signatures $[1,0]$ and $[0,1]$ meet by $\neq_{2}$, this results in 0 for the overall Holant value.) This process of "using up" the pinning signatures $[1,0]$ 's and $[0,1]$ 's must terminate, even though some new ones may be created in the process, because the sum of arities of all the signatures remaining monotonically decreases. Note that if $d=3$ then ExACTONE $d-1$ is just $\neq_{2}$ on RHS, which combined with its adjacent two copies of $\neq_{2}$ of LHS, is equivalent to a single $\neq_{2}$ of LHS. Finally, whenever an EXACTONE $_{d}$ and another EXACTONE $\ell$ are connected by a $\neq_{2}$, we replace it by a single EXACTONE $_{d+\ell-2}$, shrinking the edge between (and remove the connecting $\neq_{2}$ ). After these steps, we may assume that all pinning signatures $[1,0]$ and $[0,1]$ are eliminated, our instance graph $G$ of Pl-Holant $\left(\neq_{2} \mid={ }_{k}, \mathcal{E O}\right)$ has no self loops by $\neq_{2}$ on RHS signatures, and no $\neq_{2}$ connects two $\mathcal{E O}$ signatures on RHS.

Next we define an $E_{k}$-block. If we view every $\neq_{2}$ in the LHS of the bipartite graph $G$ as an edge, then we get a graph $G^{\prime}$ on the RHS vertices of $G$ only. Every vertex in $G^{\prime}$ is labeled by $=_{k}$ or some EXACTONE $d$, and every edge is labeled by $\neq_{2}$. The 
vertices of $G^{\prime}$ labeled by $={ }_{k}$ form connected components, with edges labeled by $\neq_{2}$. All edges in $G^{\prime}$ from such a connected component to the rest of $G^{\prime}$ (if the rest is nonempty) must connect to some $\mathcal{E} \mathcal{O}$ signatures. We define an $E_{k}$-block to be such a connected component of $=_{k}$ vertices, including the outgoing edges (if any) with all edges (including the outgoing edges) labeled by $\neq_{2}$. We call these outgoing edges of an $E_{k}$-block dangling edges. Notice that the signature defined by an $E_{k}$-block has either exactly two or zero support vectors. This depends on whether or not there exists a contradiction, which is formed by an odd cycle of $=_{k}$ connected by $\neq_{2}$, i.e., a sequence of odd number of vertices labeled by $={ }_{k}$ connected by edges labeled by $\neq_{2}$ in $G^{\prime}$. We say an $E_{k}$-block is trivial if it has no support. This is easy to check. The two support vectors of a nontrivial $E_{k}$-block are complements of each other. We mark dangling edges of a nontrivial $E_{k}$-block by "+" or "-" signs. Dangling edges marked with the same sign take the same value on both support vectors while dangling edges marked with different signs take opposite values on both support vectors. This marking is unique except one may exchange all \pm with $\mp$. The marking uniquely determines the signature of an $E_{k}$-block. Let $n_{+}$and $n_{-}$be the numbers of dangling edges marked + and - respectively. Then it is easy to see that

$$
n_{+} \equiv n_{-} \quad \bmod k \text {. }
$$

An example of $E_{6}$-block is illustrated in Fig. 18, with $8+$ signs and $2-$ signs.

Recall that we have eliminated all edges (labeled by $\neq_{2}$ ) between $\mathcal{E O}$ signatures. Thus if we define the graph between $\mathcal{E} \mathcal{O}$ signatures on the one hand and $E_{k}$-block's on the other hand, we have a bipartite (multi)-graph. Since we consider the dangling edges of any $E_{k}$-block as part of the $E_{k}$-block, we get a bipartite (multi)-graph where edges are labeled by $\left(=_{2}\right)$. This bipartite graph may have multiple edges between a pair of $E_{k}$-block and EXACTONE $d$ signatures. More importantly, we claim that it is a planar bipartite (multi)-graph.

Formally we define a contraction process on the underlying planar connected (multi)-graph for an $E_{k}$-block (which includes the dangling edges). Recursively, pick any non-dangling non-loop edge $e$, we shrink it to a point, maintaining planarity. The local cyclic orders of incident edges of the two vertices of $e$ are spliced along $e$ to form the cyclic order of the new vertex. For any self loop created we simply remove it. This contraction process ends in a single point with a cyclic order of the dangling edges.

After this contraction, we obtain a planar bipartite (multi)-graph connected between $\mathcal{E O}$ signatures and $E_{k}$-block's by edges labeled by $=2$. Note that the

Fig. 18 Example of an $E_{6}$-block. Circle vertices are assigned $=6$ and square vertices are assigned $\neq_{2}$. For simplicity, we omit the square $\neq_{2}$ on the 10 dangling edges

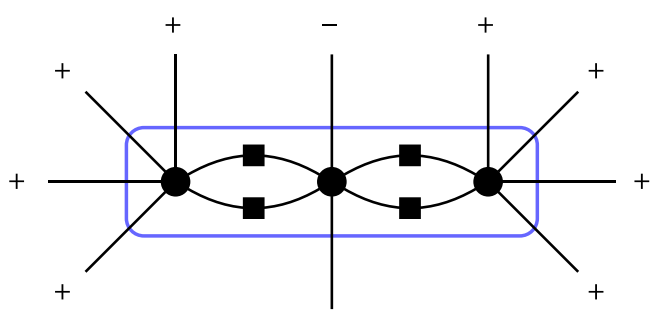

Springer 
connection becomes $=_{2}$ because the dangling edges (labeled by $\neq_{2}$ ) of an $E_{k}$-block are considered as part of the $E_{k}$-block which is now represented by a single vertex after the contraction.

The following Lemma 7.6 is a key observation that for a planar bipartite (multi)graph, if its degrees are large, then it cannot be simple, i.e., it must have parallel edges.

Lemma 7.6 Let $G=(L \cup R, E)$ be a nonempty planar bipartite (multi)-graph with parts $L$ and $R$. If every vertex in $L$ has degree at least 6 and every vertex in $R$ has degree at least 3 , then $G$ is not simple.

Proof We may assume $G$ is connected; otherwise we prove the lemma for a connected component of $G$. For a contradiction, suppose $G$ is simple. Let $v, e$ and $f$ be the total number of vertices, edges, and faces, respectively. Let $v_{i}$ be the number of vertices of degree $i$ in $L$, where $i \geq 6$, and $u_{j}$ be the number of vertices of degree $j$ in $R$, where $j \geq 3$. Since $G$ is simple and bipartite, each face has at least 4 edges. Thus,

$$
2 e \geq 4 f \text {. }
$$

Furthermore, it is easy to see that

$$
v=\sum_{i \geq 6} v_{i}+\sum_{j \geq 3} u_{j} \quad \text { and } \quad e=\sum_{i \geq 6} i v_{i}=\sum_{j \geq 3} j u_{j} .
$$

Then starting from Euler's characteristic equation for planar graphs, we have

$$
\begin{aligned}
2 & =v-e+f \\
& \leq v-\frac{e}{2} \\
& =\sum_{i \geq 6} v_{i}+\sum_{j \geq 3} u_{j}-\frac{1}{6} \sum_{i \geq 6} i v_{i}-\frac{1}{3} \sum_{j \geq 3} j u_{j} \\
& =\sum_{i \geq 6} \frac{6-i}{6} v_{i}+\sum_{j \geq 3} \frac{3-j}{3} u_{j} \leq 0,
\end{aligned}
$$

a contradiction. Here we used the trivial fact that $1 / 2=1 / 6+1 / 3$.

Lemma 7.6 does not give us tractability for the case of $k \geq 6$ yet. The reason is that given an instance of Pl-Holant $\left(\neq_{2} \mid={ }_{k}, \mathcal{E O}\right)$, after the preprocessing and forming $E_{k}$-blocks to make the graph bipartite, it is possible to have $E_{k}$-blocks of arity less than 6, in which case Lemma 7.6 does not apply. However, for $k \geq 6$ and a nontrivial $E_{k}$-block of arity $n$ where $n<6$, by (7.9) and the fact that $0 \leq n_{+}, n_{-} \leq n<k$, we see that $n_{+}=n_{-}$, and $n=n_{+}+n_{-}$must be even. If $k=5$, and $n<5$, we also have $n$ must be even. Moreover, if $n=2$, then this means that the $E_{k}$-block is just $\neq_{2}$. This $\neq_{2}$ connects $\mathcal{E O}$ signatures; if it is a self loop on a single EXACTONE E $_{d}$ signature this creates $[1,0]^{\otimes(d-2)}$, i.e., $d-2$ pinning signatures which can be handled as before, if it connects two signatures from $\mathcal{E} \mathcal{O}$ this creates a new $\mathcal{E} \mathcal{O}$ signature. The problematic case is when $n=4$. 
Figure 19a depicts the two possibilities of $E_{k}$-blocks of arity 4 up to a rotation. An $E_{k}$-block of arity 4 can be viewed as a pair of $\neq_{2}$ in parallel, but there is a correlation between them, namely their support vectors are paired up in a unique way. If we replace the contracted $E_{k}$-block of arity 4 by two parallel edges as indicated in Fig. 19 b, one can revert back to a planar realization in the $E_{k}$-block as it connects to the rest of the graph. This can be seen by reversing the contraction process step by step. Alternatively we can just use the following direct construction of $E_{k}$-blocks of arity 4 for the two types in Fig. 19a. The two types are denoted by their circular markings ++-- and +-+- . If we connect two copies of $={ }_{k}$ by $\neq$, we get the signature represented by the marking $+\cdots+-\cdots-$ with $k-1$ consecutive + 's and -'s. Then we connect the middle $k-3 \geq 0$ pairs of + and - by $\neq$ to get ++-- . For +-+- , we start from ++-- , and turn the middle + to $k-1$ consecutive - 's by connecting another copy of $=_{k}$ using $\neq$. Similarly we can turn the middle - to $k-1$ consecutive + 's. So we get $+-\cdots-+\cdots+-$ where there are $k-1$ consecutive - 's and + 's. Then we cancel the middle $k-2$ pairs of \pm by connecting $\neq$ to get +-+- . Both constructions are completed by adding an extra $\neq$ on all the dangling edges.

We will show in the following lemma how to replace $E_{k}$-blocks of arity 4 by some other signatures while keeping track of the Holant value. We also observe that the tractable set described in Lemma 7.7 allows binary $\neq_{2}$ and unary $[1,0]$ and $[0,1]$ signatures in addition to $\mathcal{E O}$ and $=_{k}$.

Lemma 7.7 For any integer $k \geq 6$, Pl-Holant $\left(\neq_{2} \mid=_{k}, \mathcal{E O}, \neq_{2},[1,0],[0,1]\right)$ is tractable.

Proof Let $\Omega$ be an instance of Pl-Holant $\left(\neq_{2} \mid={ }_{k}, \mathcal{E O}, \neq_{2},[1,0],[0,1]\right)$. Without loss of generality, we assume that $\Omega$ is connected. Any occurrence of $\neq_{2}$ of the right hand side can be removed as follows: It is connected to two adjacent copies of $\neq_{2}$ of the left hand side. We replace this chain of three copies of $\neq_{2}$ by a single $\neq_{2}$ from the left hand side.

The given signatures have no weight, however the proof below can be adapted to the weighted case. For the unweighted case, we only need to count the number of satisfying assignments. We call an edge in the bipartite instance graph of $\Omega$ pinned if it has the same value in all satisfying assignments, if there is any. Clearly any edge incident to a vertex assigned [1,0] or [0, 1] is pinned.

When an edge is pinned to a known value, we can get a smaller instance of the problem Pl-Holant $\left(\neq_{2} \mid=_{k}, \mathcal{E O}, \neq_{2},[1,0],[0,1]\right)$ without changing the number of

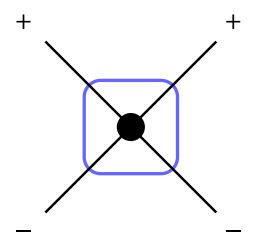

(a)

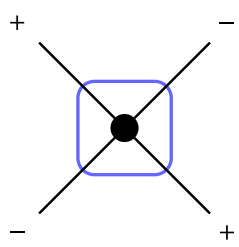

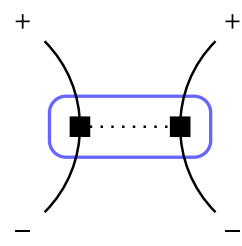

(b)

Fig. 19 Arity $4 E_{k}$-blocks. a Two dierent arity $4 E_{k}$-blocks. b Replace them by parallel $\neq_{2}$ 's 
satisfying assignments. In our algorithm we may also find a contradiction and simply return 0 . If $e$ is a pinned edge, then it is adjacent to another edge $e^{\prime}$ via $\neq 2$ on the left hand side, and both $e$ and $e^{\prime}$ are pinned. We remove $e, e^{\prime}$, and $\neq_{2}$, and perform the following on $e$ (and on $e^{\prime}$ as well). If the other endpoint of $e$ is $u=[1,0]$ or $[0,1]$ we either remove that $u$ if the pinned value on $e$ is consistent with $u$, or return 0 otherwise. If the other endpoint of $e$ is $=k$, then all edges of this $=_{k}$ are pinned to the same value which we can recursively apply. If the other endpoint of $e$ is EXACTONE $_{d} \in \mathcal{E O}$, then we replace this signature by EXACTONE $_{d-1}$ when the pinned value is 0 ; or if the pinned value is 1 then the remaining $d-1$ edges of this EXACTONE $_{d}$ are pinned to 0 which we recursively apply. Notice that we may create an $\operatorname{EXACTONE}_{2}\left(\right.$ i.e. $\neq \neq_{2}$ ) on the right hand side when we pin 0 on EXACTONE 3 . Such $\neq 2$ 's are replaced as described at the beginning. It is easy to see that all these steps do not change the number of satisfying assignments, and work in polynomial time.

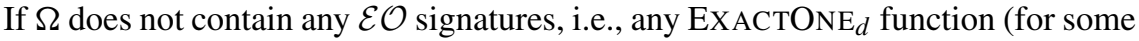
$d \geq 3$ ), then $\Omega$ is an instance of $\mathscr{P}$ and therefore tractable. If $\Omega$ does not contain any $=_{k}$, then it is an instance of $\mathscr{M}$ and therefore also tractable. So we may assume $\Omega$ contains at least one $=_{k}$ and at least one $\mathcal{E} \mathcal{O}$ signature. Then, we claim that there always exists an edge in $\Omega$ that is pinned, or there is a contradiction. Furthermore, in polynomial time we can find a pinned edge with a known value, or return that there is a contradiction. (If there is a contradiction in $\Omega$, we may still return a purported pinned edge with a known value, which we can apply and simplify $\Omega$. The contradiction will eventually be found.) The lemma follows from the claim, for we either recurse on a smaller instance or have a tractable instance.

Now we assume $\Omega$ is an instance where at least one $=_{k}$ and at least one EXACTONE $_{d} \in \mathcal{E O}$ appear. We assume no $\neq_{2}$ appears on the right hand side. If any $[1,0]$ or $[1,0]$ appear, then we have found a pinned edge with a known value. Hence we may assume neither $[1,0]$ nor $[1,0]$ appears in $\Omega$. So we may assume $\Omega$ is an instance for Pl-Holant $\left(\neq_{2} \mid={ }_{k}, \mathcal{E O}\right)$.

If a signature EXACTONE $d \in \mathcal{E O}$ is connected to itself by a self-loop through a $\neq_{2}$, then there are two choices for the assignment on this pair of edges through the $\neq_{2}$, but the remaining $d-2 \geq 1$ edges are pinned to 0 . We can keep track of the factor 2 and have found a pinned edge with a known value. Thus we may assume there are no self-loops via $\neq_{2}$ on $\mathcal{E O}$ signatures.

Next we consider the case that two separate (occurrences of) signatures from $\mathcal{E} \mathcal{O}$, say, EXACTONE $_{d}$ and EXACTONE, are connected by some number of $\neq \neq_{2}$ 's. (Here $d=\ell$ is permitted.) Depending on the number of connecting edges, there are three cases:

1. The connection is by a single $\neq_{2}$. We contract the connecting edge, maintaining planarity, and replace these three signatures by an EXACTONE $_{d+\ell-2}$ to get a new instance $\Omega^{\prime}$. If an edge is pinned in $\Omega^{\prime}$ then it is also pinned in $\Omega$ to the same value. We continue with $\Omega^{\prime}$.

2. The connection is by two $\neq_{2}$ 's. There are two choices for the assignment on these two pairs of edges through $\neq_{2}$, but the remaining $d+\ell-4 \geq 2$ edges are pinned to 0 .

3. The connection is by at least three $\neq_{2}$ 's. The three $\neq_{2}$ 's cannot be all satisfied, so there is no satisfying assignment, a contradiction. We return the value 0 . 
Hence, we may assume there is no connection via any number of $\neq 2$ 's among $\mathcal{E O}$ signatures.

We can now use our definition of an $E_{k}$-block (before Lemma 7.6) as a connected component composed of $=_{k}$ and $\neq_{2}$. All external connecting edges of each $E_{k}$-block are marked with + or - and this can be found by testing bipartiteness of an $E_{k}$-block where we treat $\neq 2$ 's as edges and $=_{k}$ 's as vertices. If any $E_{k}$-block is not bipartite, we return 0 . Such an $E_{k}$-block is called trivial. In the following we may assume no trivial $E_{k}$-block exists. We contract all $E_{k}$-blocks and maintain planarity, as described earlier: For each $E_{k}$-block we contract two vertices that are connected by an edge, one edge at a time, and remove self loops in this contraction process. Any nontrivial $E_{k}$-block of arity 2 has signature $\neq_{2}$, by (7.9). If there is a nontrivial $E_{k}$-block of arity 2 , we replace it with an edge labeled by $\neq_{2}$ to form an instance $\Omega^{\prime}$, maintaining planarity, such that any pinned edge in $\Omega^{\prime}$ corresponds to a pinned edge in $\Omega$. This new edge is between $\mathcal{E} \mathcal{O}$ signatures and can be dealt with as described earlier. So we may assume the arity of any $E_{k}$-block is at least 4 . Since $k \geq 6$, the only possible $E_{k}$-blocks of arity 4 are those in Fig. 19 a up to a rotation. Since there is at least one EXACTONE ${ }_{d}$ signature with $d \geq 3$, forming $E_{k}$-blocks does not consume all of $\Omega$.

After these steps we may consider $\Omega$ a bipartite graph, with one side consisting of $E_{k}$-blocks and the other side $\mathcal{E} \mathcal{O}$ signatures. And they are now connected by edges labeled by $={ }_{2}$.

Suppose there are parallel edges between an $E_{k}$-block and an EXACTONE $_{d}$ signature. We show that this always leads to some pinned edges. If two parallel edges are marked by the same sign in the $E_{k}$-block, then they must be pinned to 0 . If they are marked by different signs, then the remaining $d-2 \geq 1$ edges of the EXACTONE $d$ signature must be pinned to 0 . Therefore, we may assume there are no parallel edges between any $E_{k}$-block and any $\mathcal{E} \mathcal{O}$ signature.

The next thing we do is to consider $E_{k}$-blocks of arity 4 with $\mathcal{E O}$ signatures together. Call a connected component consisting of $E_{k}$-blocks of arity 4 and $\mathcal{E O}$ signatures an EO-Eq-4-block. Figure 20a illustrates an example. Notice that the two

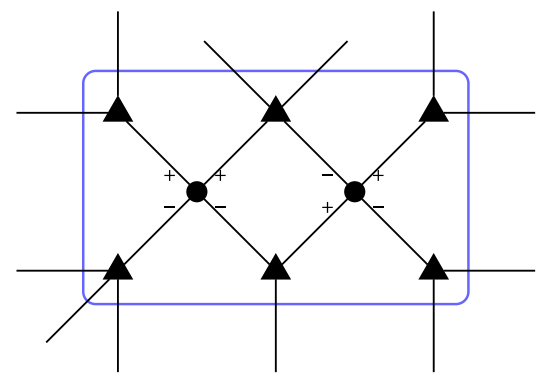

(a)

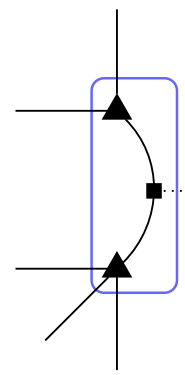

(b)

Fig. $20 E O$-Eq-4-blocks. a An $E O-E q$-4-block. Triangles are assigned $\mathcal{E O}$ signatures and circles are $E_{k}$-blocks of arity 4 . b Break the $E O$ - $E q$-4-block into three components. Squares are assigned $\neq 2$. The component in the middle contains a cycle, and hence is degenerate. The other two are equivalent to $\mathcal{E} \mathcal{O}$ signatures 
possibilities of $E_{k}$-blocks of arity 4 can be viewed as two parallel $\neq 2$ 's but with some correlation between them. This is illustrated in Fig. 19b. Note that the two dotted lines in Fig. 19b represent different correlations.

At this point we would like to replace every $E_{k}$-block of arity 4 by two parallel $\neq 2$ 's. However this replacement destroys the equivalence of the Holant values, before and after.

The surprising move of this proof is that we shall do so anyway!

Suppose we ignore the correlation for the time being and replace every $E_{k}$-block of arity 4 by two parallel $\neq_{2}$ 's (each parallel copy of $\neq_{2}$ is technically a path of length 2 , with the middle vertex labeled by $\neq_{2}$ ) as in Fig. 19b. This replacement produces a planar signature grid $\Omega_{1}$. Every edge in $\Omega_{1}$ corresponds to a unique edge in $\Omega$. The set of satisfying assignments of $\Omega_{1}$ is a superset of that of $\Omega$. Moreover, if there is an edge pinned in $\Omega_{1}$ to a known value, the corresponding edge is also pinned in $\Omega$ to the same value. Once we find that in $\Omega_{1}$ we revert back to work in $\Omega$ and apply the pinned value to the pinned edge.

All that remains to be shown is that pinning always happens in $\Omega_{1}$. Each EOEq-4-block splits into some number of connected components in $\Omega_{1}$. Suppose some component contains a cycle. Such a cycle must alternate between $\neq_{2}$ (these are the newly created ones from splitting the $E_{k}$-blocks of arity 4) and EXACTONE $d$ signatures for $d \geq 3$ (the cycle may involve only one EXACTONE $d$ and one $\neq_{2}$, in which case the cycle has length 2 ). Each cycle has even length (as each copy of $\neq_{2}$ takes length 2), and there are exactly two potential satisfying assignments, which assign exactly one 0 and one 1 to the two cycle edges incident to each EXACTONE $_{d}$ signature. Then any edge not on the cycle but incident to some vertex in the cycle is pinned to 0 . Such edges must exist, for EXACTONE $d$ signatures in the cycle are of arity at least 3 . Thus we have found pinned edges.

Hence we may assume there are no cycles in these components, and every such component forms a tree. We can view this tree as a tree on vertices labeled by $\mathcal{E O}$ functions and edges by $\neq_{2}$ 's. Since we have at least one copy of $\neq_{2}$ in each component, and it does not form a loop on an $\mathcal{E O}$ signature, the tree has $n \geq 2$ vertices, and $n-1$ edges. Similar to the discussion in item 1 above for connecting two $\mathcal{E O}$ functions EXACTONE $_{d}$ and EXACTONE $\ell$ by a single $\neq_{2}$, the whole tree is an EXACTONE $_{t}$ function for some arity $t$. Since each vertex in the tree has degree at least 3 , the arity $t \geq 3 n-2(n-1)=n+2 \geq 4$. We replace these components by EXACTONE $t$ 's.

Thus, each connected component in the graph underlying $\Omega_{1}$ is a planar bipartite graph with $E_{k}$-blocks of arity at least 6 on the one side and $\mathcal{E O}$ signatures on the other. By Lemma 7.6, no component is simple, which means that there are parallel edges between some $E_{k}$-block and some $\mathcal{E} \mathcal{O}$ signature. As discussed earlier, there must exist some pinned edge, and we can find a pinned edge with a known value in polynomial time. This finishes the proof.

As indicated in the proof, Lemma 7.7 holds for the weighted version of the signature sets as well. As defined in Section 2, a weighted binary disequality $(0, a, b, 0)$ (in truth table notation) outputs $a$ and $b$ on input 01 and 10 respectively. A weighted $=_{k}$ has signature $[c, 0, \ldots, 0, d]$ of arity $k$, for some $c, d$. Weighted pinning signatures 
are scalar multiples of $[1,0]$ and $[0,1]$. Finally, weighted $\mathcal{E O}$ signatures are of the form EXACTONE ${ }_{k: a_{1}, \ldots, a_{k}}$ defined in Section 3, where $k \geq 3$.

We now briefly describe the modifications needed to prove Lemma 7.8, the weighted version of Lemma 7.7. First, the removal of weighted $\neq_{2}$ from the RHS still works provided we keep track of the resulting weighted $\neq_{2}$ on the RHS after combining a chain of three weighted $\neq 2$ 's. Next for a pinned edge $e$ connected by a weighted $\neq_{2}$ on the LHS with another edge $e^{\prime}$, we obtain a factor depending on the pinned value is 0 or 1 , and $e^{\prime}$ is also pinned to the same value as $e$. The update on the weighted $={ }_{k}$ or weighted $\mathcal{E O}$ signatures that are connected to $e$ and $e^{\prime}$ on the RHS can be done similarly, keeping track of a multiplicative factor.

If a weighted $\mathcal{E O}$ signature EXACTONE $k_{k} c_{1}, \ldots, c_{k}$ has its $i$ th and $j$ th variables connected to the 1 st and 2 nd variables of a weighted $\neq_{2}$ signature $(0, a, b, 0)$, we obtain

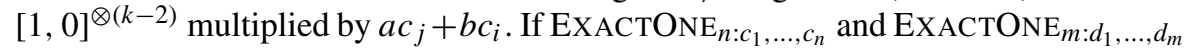
are connected by $(0, a, b, 0)$, for notational simplicity we may assume the $n$th variable of EXACTONE ${ }_{n: c_{1}, \ldots, c_{n}}$ is connected to the 1 st variable of $(0, a, b, 0)$, and the $m$ th variable of EXACTONE $m: d_{1}, \ldots, d_{m}$ to the second. Then we obtain a weighted version of EXACTONE $\mathrm{E}_{n+m-2}$, where the parameters are $a c_{i} d_{m}$ for $1 \leq i \leq n-1$ and $b c_{n} d_{j-n+1}$ for $n \leq j \leq n+m-2$.

Weighted versions of $E_{k}$-block are defined in the same way, except there will be two factors $(a, b)$ associated to it: When all + dangling edges are assigned 1 and all - dangling edges are assigned 0 , we get value $a$, and in the complement assignment we get value $b$.

Finally when we split an $E_{k}$-block of arity 4 into two parallel $\neq_{2}$ 's, we do not have to keep track of the weight of the $E_{k}$-block of arity 4, because the purpose of that split is just to find a pinned edge. When such a pinned edge is found we revert back from $\Omega_{1}$ to $\Omega$ and continue there.

These observations lead to:

Lemma 7.8 Lemma 7.7 holds when each indicated signature is replaced by any set of weighted versions of the signature of the same type.

Unlike the situation in Lemma 7.6, a planar (5,3)-regular bipartite graph can be simple. However, we show that any planar simple bipartite graph where the degree from one side is at least 5 and the degree from the other side is at least 3 must have a special induced subgraph. We call this structure a "wheel", which is pictured in Fig. 21. In this wheel structure, there is a vertex $v$ of degree 5 in the middle, and all faces adjacent to this vertex are 4-gons (i.e. quadrilaterals). Moreover, at least four neighbors of $v$ have degree 3. Depending on whether the degree of the fifth neighbor is 3 or not, we have two types of wheel, which are pictured in Fig. 21a and b.

Lemma 7.9 Let $G=(L \cup R, E)$ be a planar bipartite graph with parts $L$ and $R$. Every vertex in $L$ has degree at least 5 and every vertex in $R$ has degree at least 3 . If $G$ is simple, then there exists one of the two wheel structures in Fig. 21 as an induced subgraph in $G$ (where the five occurrences of the circle vertices on the outer circuit of length 10 may not be all distinct). 


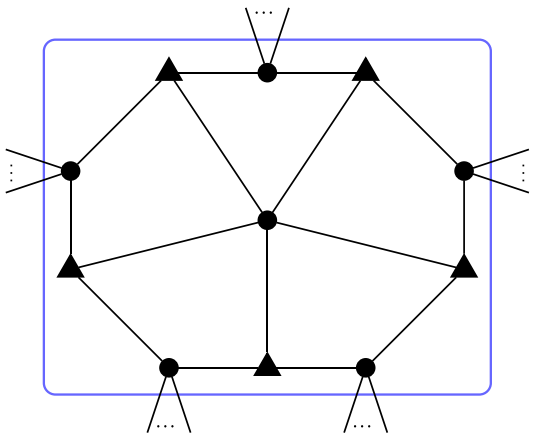

(a)

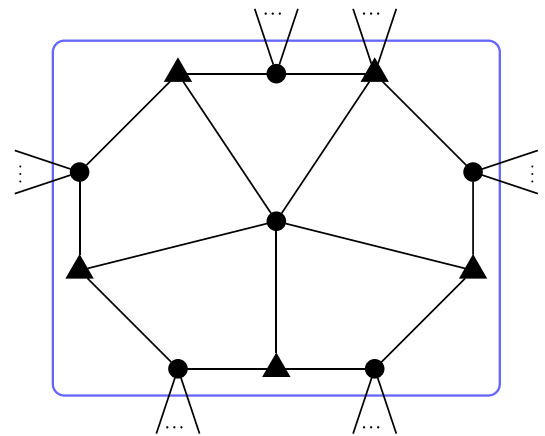

(b)

Fig. 21 Two types of wheels. Each circle is an $E_{5}$-block and each triangle is an $\mathcal{E} \mathcal{O}$ signature. The five occurrences of circle vertices may be nondistinct. The five triangle vertices are all distinct, and the outer circuit of length 10 is edge disjoint. a Type 1 . b Type 2

Proof Let $V=L \cup R$ be the set of vertices and let $F$ be the set of faces. We assign a score $s_{v}$ to each of its vertices $v \in V$. We will define $s_{v}$ so that $\sum_{v \in V} s_{v}=|V|-$ $|E|+|F|=2>0$. For each $v \in V$, the score $s_{v}$ is the sum of the following three parts:

1. For being a vertex, it gets value 1 . This accounts for $|V|$ over all $v \in V$.

2. For each face, we call it a $k$-gon if we encounter $k$ occurrences of edges (and $k$ occurrences vertices) if we traverse the face in the interior clockwise. For each $k$ gon face, and each occurrence of $v$ on it, we assign $\frac{1}{k}$ to $v$. Overall, this accounts for $|F|$.

3. For $-|E|$, we separate two cases. As $G$ is simple and bipartite, there is no self loop. For any edge if one of the two endpoints has degree 3, we give the degree 3 vertex a score of $-\frac{7}{12}$, and the other one $-\frac{5}{12}$. This is well defined because all degree 3 vertices are in $R$. If the endpoints are not of degree 3 , we give each endpoint $-\frac{1}{2}$. This accounts for $-|E|$.

Note that since $G$ is bipartite, every $k$-gon face has $k$ even. And since $G$ has no parallel edges, we have $k>2$. Hence $k \geq 4$. Also, the number of occurrences $v$ appears when we traverse all faces is the degree of $v$. Thus the total score for $v$ coming from all faces is at most $\frac{\operatorname{deg}(v)}{4}$. Now we have the following claim.

Claim: $\quad s_{v} \leq 0$ unless $v \in L$ and $\operatorname{deg}(v)=5$.

To prove the claim, first suppose $v \in L$ and has degree $d \geq 6$. Then

$$
s_{v} \leq 1+\frac{d}{4}-\frac{5}{12} d=1-\frac{d}{6} \leq 0 .
$$

Next, suppose $v \in R$ and $v$ has degree $d \geq 4$. Then every edge incident to $v$ gives a score $-\frac{1}{2}$. Hence,

$$
s_{v} \leq 1+\frac{d}{4}-\frac{1}{2} d=1-\frac{d}{4} \leq 0 .
$$


The remaining case is that $v \in R$ and $v$ has degree 3 . Then,

$$
s_{v} \leq 1+\frac{d}{4}-\frac{7}{12} d=1-\frac{d}{3} \leq 0 .
$$

The claim is proved.

Since the total score is positive, there must exist $v \in L, \operatorname{deg}(v)=5$, and $s_{v}>0$. Because $G$ is bipartite and simple, $v$ and its five neighboring vertices $v_{1}, \ldots, v_{5}$ are six distinct vertices.

We then claim that there must exist such a $v$ so that all five occurrences of adjacent faces are 4-gons. Suppose otherwise. Then for any such $v$, among the five occurrences of faces adjacent to $v$, at least one occurrence of a $k$-gon has $k \geq 6$. In this case,

$$
s_{v} \leq 1+\frac{1}{4} \cdot 4+\frac{1}{6}-\frac{5}{12} \cdot 5=\frac{1}{12} .
$$

Moreover, if $v$ is adjacent to more than one occurrences of $k$-gons with $k \geq 6$, Then

$$
s_{v} \leq 1+\frac{1}{4} \cdot 3+\frac{1}{6} \cdot 2-\frac{5}{12} \cdot 5=0
$$

contrary to the assumption that $s_{v}>0$. Hence $v$ is adjacent to exactly one occurrence of $k$-gon with $k \geq 6$-we will call it $F_{v}$-and all other occurrences are 4-gons. Clearly $F_{v}$ is distinct from the 4-gons. Moreover these four occurrences of 4-gons are all distinct 4 -gons. This is because, by cyclically renaming $v_{1}, \ldots, v_{5}$, we may name $\left\{v, v_{i}, v_{i+1}, u_{i}\right\}$ for $1 \leq i \leq 4$ the vertices of the four occurrences of 4-gons. If there are any multiple occurrences of these 4-gons, by $G$ being bipartite we would have $\left\{v_{i}, v_{i+1}\right\}=\left\{v_{j}, v_{j+1}\right\}$ for some $1 \leq i<j \leq 4$. This is a contradiction since $\left\{v_{1}, \ldots, v_{5}\right\}$ are five distinct vertices.

In $F_{v}, v$ has two neighbors in $R$. We associate each vertex $v$ that has a positive score to the vertex on $F_{v}$ that is the next one in clockwise order from $v$. By bipartiteness, every such $v$ is associated to a vertex in $R$. We do this association in all occurrences of faces containing at least one positively scored vertex. It is possible that more than one such $v$ are associated to the same $u \in R$. Suppose a vertex $u \in R$ is associated to from $\ell$ different such vertices of positive score. This means that $u$ is adjacent to at least $\ell$ many occurrences of $k$-gons with $k \geq 6$. Note that $\ell \leq \operatorname{deg}(u)$. Then, if $u$ has degree 3 then $0 \leq \ell \leq 3$, and $u$ has score

$$
s_{u} \leq 1+\frac{1}{4} \cdot(3-\ell)+\frac{1}{6} \cdot \ell-\frac{7}{12} \cdot 3=-\frac{\ell}{12} .
$$

If $u$ has degree $d \geq 4$ then $0 \leq \ell \leq d$, and $u$ has score

$$
s_{u} \leq 1+\frac{1}{4} \cdot(d-\ell)+\frac{1}{6} \cdot \ell-\frac{1}{2} \cdot d \leq-\frac{\ell}{12} .
$$

Hence in any case, we have $s_{u} \leq-\frac{\ell}{12}$. This implies that the total score of $u$ and all positively scored vertices associated to $u$ is at most 0 . However each positively scored vertex is associated to a vertex in $R$. Hence the total score cannot be positive. This is a contradiction.

Therefore there exists $v \in L$ such that $s_{v}>0$, and has degree 5, and all adjacent occurrences of faces are 4-gons. Then we can name these five occurrences of 4-gons to have vertex sets $\left\{v, v_{i}, v_{i+1}, u_{i}\right\}$ for $1 \leq i \leq 5$ (where we name $v_{6}$ as $v_{1}$ ). Again, by $G$ being bipartite and $\left\{v_{1}, \ldots, v_{5}\right\}$ being five distinct vertices, these five 4-gons are all distinct. (We do not rule out the possibility that $u_{1}, \ldots, u_{5}$ may be not all 
distinct; however, because $G$ is simple, all $u_{1}, \ldots, u_{5}$ are distinct from $v$. Thus, in the two wheels depicted in Fig. 21a and b, among the 11 occurrences of vertices which we just named $v, v_{1}, \ldots, v_{5}, u_{1}, \ldots, u_{5}$, only $u_{1}, \ldots, u_{5}$ may be nondistinct. Since $v_{1}, \ldots, v_{5}$ are distinct, the outer circuit of length 10 is edge disjoint.) We further note that at most one neighbor of $v$ can have degree $\geq 4$, for otherwise,

$$
s_{v} \leq 1+\frac{5}{4}-\frac{1}{2} \cdot 2-\frac{5}{12} \cdot 3=0
$$

If all neighbors of $v$ have degree 3, that is a wheel of type 1 as in Fig. 21a. If one neighbor of $v$ has degree $\geq 4$, that is a wheel of type 2 as in Fig. 21 b.

As we shall see, either structure in Fig. 21 leads to pinned edges.

Lemma 7.10 Pl-Holant $\left(\neq_{2} \mid={ }_{5}, \mathcal{E O}, \neq_{2},[1,0],[0,1]\right)$ is tractable.

Proof We proceed as in Lemma 7.7 up until the point of getting $\Omega_{1}$. Note that due to (7.9) the only nontrivial $E_{5}$-blocks of arity $\leq 4$ are $\neq_{2}$ and those in Fig. 19a. Moreover, each connected component of $\Omega_{1}$ is planar and bipartite with vertices on one side having degree at least 5 and those on the other at least 3 . We only need to show that there are edges pinned in $\Omega_{1}$.

Unlike in Lemma 7.7, the underlying graph of $\Omega_{1}$ does not satisfy the condition of Lemma 7.6 but that of Lemma 7.9. If the graph is not simple, then there are pinned edges similar to Lemma 7.7. Otherwise by Lemma 7.9, the wheel structure in Fig. 21 appears. Note that by (7.9), $n_{+} \equiv n_{-} \bmod 5$ and the arity $n_{+}+n_{-}=5$ imply that the center vertex, which is an $E_{5}$-block, has signature $={ }_{5}$. All we need to show is that wheel structures of either type contain pinned edges.

First we claim that if a wheel of either type has a $E_{5}$-block, call it $E_{1}$, on the outer circuit that has different sign labels $(+/-)$ on the two edges incident to it along the circuit, then the center vertex denoted by $E_{o}$ and has signature $=5$, is pinned. This is pictured in Fig. 22a. It does not matter whether the wheel is type 1 or 2, or the position

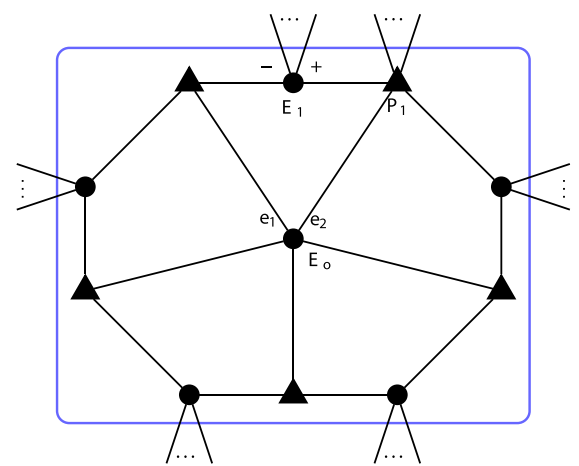

(a)

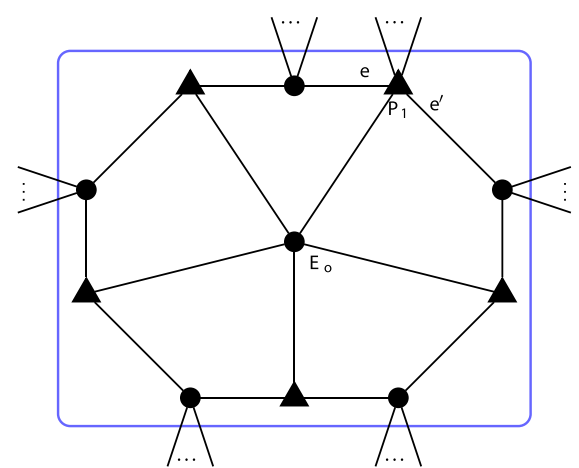

(b)

Fig. 22 Degeneracies in the wheel structure. a Different signs of an $E_{5}$-block along the outer circuit lead to pinning. $\mathbf{b}$ Edges $e$ and $e^{\prime}$ are pinned in wheels of type 2 
of $E_{1}$ relative to the special triangle $P_{1}$ in type 2 . Because $E_{o}$ is an equality, both $e_{1}$ and $e_{2}$, the two edges incident to $E_{o}$ that are connected to the two $\mathcal{E O}$ signatures flanking $E_{1}$, must take the same value. If both $e_{1}$ and $e_{2}$ are assigned 1 , then the two incoming wires of $E_{1}$ along the circuit have to be both assigned 0 , whereas they are marked by different signs. This is a contradiction. Hence both $e_{1}$ and $e_{2}$ are pinned to 0 as well as all edges incident to $E_{o}$.

We may therefore assume that each $E_{5}$-block has equal signs along the outer circuit, either ++ or -- (some may have ++ while others may have -- ). Then as far as the two edges along the outer circuit incident to each $E_{5}$-block are concerned, each $E_{5}$-block serves as a binary equality; this is true regardless whether the five triangle vertices for $E_{5}$-block's along the outer circuit in Fig. 21 are distinct or not.

Suppose the wheel has type 1 . If the edges incident to $E_{o}$ are assigned 0 , then the five EXACTONE 3 signatures along the outer circuit have all effectively become $\neq 2$ along the circuit. If we now consider the $E_{5}$-block signatures along the outer circuit as equalities (since they have signs ++ or -- ) and treat them as edges, we get a cycle on the five EXACTONE 3 signatures along the outer circuit as a cycle of five $\neq 2$ 's. This has no satisfying assignment. Hence $E_{o}$ and all its incident edges are pinned to 1 .

Otherwise the wheel is of type 2 , and each $E_{5}$-block has signs ++ or -- along the outer circuit. We denote by $P_{1}$ the special EXACTONE $d$ function that has arity $d>3$. We claim that the two edges $e$ and $e^{\prime}$ incident to $P_{1}$ along the circuit are both pinned to 0 . This is illustrated in Fig. 22b. As $P_{1}$ is ExACTONE $d$, at most one of $e$ and $e^{\prime}$ is 1 . If one of $e$ and $e^{\prime}$ is 1 , the other is 0 , and as $P_{1}$ is an EXACTONE $_{d}$ function its edge to $E_{o}$ is also 0 , and thus all edges incident to $E_{o}$ are 0 . As all five neighbors of $E_{o}$ are $\mathcal{E O}$ functions, the four EXACTONE 3 functions effectively become $\left(\neq_{2}\right)$ functions along the wheel, and we can remove $E_{o}$ and its incident edges. This becomes the same situation as in the previous case of type 1 , where effectively a circuit of five binary equalities are linked by five binary disequalities, which has no valid assignment. This implies that both $e$ and $e^{\prime}$ are pinned to 0 . This finishes the proof.

Similar considerations as that after the proof of Lemma 7.7 lead to the weighted version of Lemma 7.10.

Lemma 7.11 Lemma 7.10 holds when each indicated signature is replaced by any set of weighted versions of the signature of the same type.

\subsection{Lemmas Related to $\mathscr{M}_{4}$ and $\mathscr{P}_{2}$}

Now we prove some lemmas relating to $\mathscr{M}_{4}$ and $\mathscr{P}_{2}$, defined in Section 3 , that are used in the proof of the full dichotomy, Theorem 8.1.

Recall that AlLBUTONE $d$ is the signature $[0, \ldots, 0,1,0]$ of arity $d$, which is the

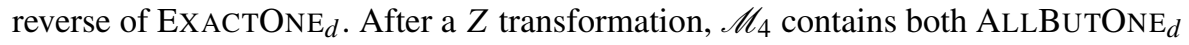
and EXACTONE $d$. However, for arity at least 3, if both appear, then with any $=_{k}$ the problem is hard. 
Lemma 7.12 If integers $d_{1}, d_{2}, k \geq 3$, then Pl-Holant $\left(\neq_{2} \mid=_{k}\right.$, EXACTONE $d_{1}$, AlLButONE $_{d_{2}}$ ) is \#P-hard.

Proof We first get the pinning signatures $[1,0]$ and $[0,1]$. We apply Lemma 7.4 to create $[0,1]$. Then note that AlLBuTONE $d$ is just flipping the roles of 0 and 1 in EXACTONE $_{d}$, we can get $[1,0]$ too. So we get both $[1,0]$ and $[0,1]$ by applying Lemma 7.4 twice. With both $[1,0]$ and $[0,1]$ in hand, we may reduce $d_{1}$ (respectively $d_{2}$ ) to 4 if $d_{1}>4$ (respectively $d_{2}>4$ ), and obtain ExACTONE4 and AlLBUTONE 4 . If either of the two arities $d_{1}$ or $d_{2}$ is 3 , then we connect two copies together via $\neq_{2}$ to realize an arity 4 copy.

Moreover, we use the gadget illustrated in Fig. 23 to create the function $\hat{g}$ in Lemma 6.7 as an $E_{k}$-block. Then by Lemma $6.7, \operatorname{Pl}$-Holant $\left(\neq_{2} \mid=_{k}\right.$, EXACTONE $_{d_{1}}$, AllButONE $d_{2}$ ) is \#P-hard.

By definition $\mathscr{P}_{2}=\mathscr{A}_{2}$, and by Lemma 3.2, signatures in $\mathscr{P}_{2}$ are non-degenerate weighted equalities under the $Z$ transformation. The next several lemmas show that the hardness criterion is the same regardless of the weight.

Lemma 7.13 Let $f \in \mathscr{P}_{2}, g_{1} \in \mathscr{M}_{4}^{+}, g_{2} \in \mathscr{M}_{4}^{-}$be non-degenerate signatures with arity $\geq 3$. Then Pl-Holant $\left(f, g_{1}, g_{2}\right)$ is \#P-hard.

Proof Suppose the arities of $f, g_{1}$, and $g_{2}$ are $n, m_{1}$, and $m_{2}$ respectively. Under a holographic transformation by $Z$, we have

$$
\begin{aligned}
\text { Pl-Holant }\left(f, g_{1}, g_{2}\right) & \equiv \text { Pl-Holant }\left(\neq_{2} \mid\left(Z^{-1}\right)^{\otimes n} f,\left(Z^{-1}\right)^{\otimes m_{1}} g_{1},\left(Z^{-1}\right)^{\otimes m_{2}} g_{2}\right) \\
& \equiv \text { Pl-Holant }\left(\neq_{2} \mid \hat{f}, \text { ExACTONE }_{m_{1}}, \text { ALLBuTONE }_{m_{2}}\right),
\end{aligned}
$$

where $\hat{f}=\left(Z^{-1}\right)^{\otimes n} f$ has the form $[1,0, \ldots, 0, c]$ up to a nonzero constant, with $c \neq 0$, as $f \in \mathscr{P}_{2}$. We do another diagonal transformation by $D=\left[\begin{array}{ll}1 & 0 \\ 0 & c^{1 / n}\end{array}\right]$. Then

Pl- Holant $\left(f, g_{1}, g_{2}\right)$

$$
\begin{aligned}
& \equiv \text { Pl-Holant }\left(\left(\neq_{2}\right) D^{\otimes 2} \mid\left(D^{-1}\right)^{\otimes n} \hat{f},\left(D^{-1}\right)^{\otimes m_{1}} \operatorname{EXACTONE}_{m_{1}},\left(D^{-1}\right)^{\otimes m_{2}} \operatorname{ALLBUTONE}_{m_{2}}\right) \\
& \equiv \text { Pl-Holant }\left(\neq_{2} \mid=_{n}, \text { EXACTONE }_{m_{1}}, \text { ALLBUTONE }_{m_{2}}\right),
\end{aligned}
$$

Fig. 23 Gadget to realize $\hat{g}$ in Lemma 7.12. Circle vertices are assigned $=_{k}$ and square vertices are assigned $\neq_{2}$. The number of parallel edges is $k-2$

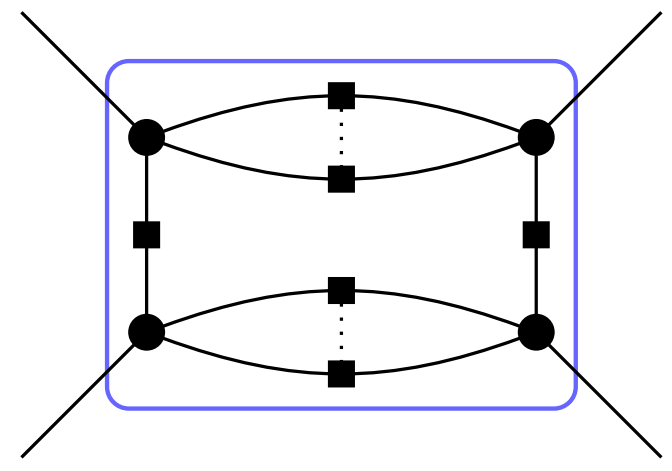


where in the last line we ignored several nonzero factors. The lemma follows from Lemma 7.12.

We also need to consider the mixture of $\mathscr{P}_{2}$ and binary signatures.

Lemma 7.14 Let $\mathcal{F}$ be a set of symmetric signatures. Suppose $\mathcal{F}$ contains a nondegenerate signature $f \in \mathscr{P}_{2}$ of arity $n \geq 3$ and a binary signature $h$. Then $\operatorname{Pl}-H o l a n t(\mathcal{F})$ is \#P-hard unless $h \in Z \mathscr{P}$, or $\operatorname{Pl}$ \#CSP${ }^{2}\left(D Z^{-1} \mathcal{F}\right) \leq_{T} \operatorname{Pl}$-Holant $(\mathcal{F})$ for some diagonal transformation $D$.

Proof We do a $Z$ transformation and get

$$
\begin{aligned}
\operatorname{Pl} \text {-Holant }(\mathcal{F}) & \equiv \operatorname{Pl-Holant}(\mathcal{F}, h, f) \\
& \equiv \text { Pl-Holant }\left(\neq_{2} \mid Z^{-1} \mathcal{F},\left(Z^{-1}\right)^{\otimes 2} h, \hat{f}\right),
\end{aligned}
$$

where $\hat{f}=\left(Z^{-1}\right)^{\otimes n} f=[1,0, \ldots, 0, t]$ up to a nonzero constant with $t \neq 0$. We further do another diagonal transformation of $D_{1}=\left[\begin{array}{ll}1 & 0 \\ 0 & t^{1 / n}\end{array}\right]$. Then

$$
\begin{aligned}
\operatorname{Pl-Holant}(\mathcal{F}) & \equiv \text { Pl-Holant }\left(\left(\neq_{2}\right) D_{1}^{\otimes 2} \mid\left(D_{1}^{-1}\right)^{\otimes n} \hat{f},\left(Z D_{1}\right)^{-1} \mathcal{F},\left(\left(Z D_{1}\right)^{-1}\right)^{\otimes 2} h\right) \\
& \equiv \text { Pl-Holant }\left(\neq_{2} \mid=_{n},\left(Z D_{1}\right)^{-1} \mathcal{F},\left(\left(Z D_{1}\right)^{-1}\right)^{\otimes 2} h\right) \\
& \geq_{T} \text { Pl-Holant }\left(=_{n} \mid\left(Z D_{1}\right)^{-1} \mathcal{F},\left(\left(Z D_{1}\right)^{-1}\right)^{\otimes 2} h\right),
\end{aligned}
$$

where in the second line we ignore a nonzero factor on $\neq_{2}$. Hence by Theorem 2.24 , Pl-Holant $(\mathcal{F})$ is \#P-hard unless $\left(\left(Z D_{1}\right)^{-1}\right)^{\otimes 2} h \in \mathscr{P}$ (cases 1,2 or 3 in Theorem 2.24) or $\left(\left(Z D_{1}\right)^{-1}\right)^{\otimes 2} h=[a, b, c]$ for some $a, b, c \in \mathbb{C}$ such that $a c \neq 0$ and $(a / c)^{2 n}=1$ (cases 4 or 5 in Theorem 2.24).

If $\left(\left(Z D_{1}\right)^{-1}\right)^{\otimes 2} h \in \mathscr{P}$, then $h \in Z D_{1} \mathscr{P}=Z \mathscr{P}$ as $D_{1} \in \operatorname{Stab}(\mathscr{P})$. In the latter case, we construct $=2 n$ on the right by connecting three copies of $=_{n}$ to one copy of $=_{n}$ via $\neq_{2}$. We do the same construction again to realize $=_{4 n}$ using $=2 n$. We connect $n-1$ many $[a, b, c]$ 's to $=2 n$ via $\neq 2$ to realize a binary weighted equality $[1,0, r]$ with $r=(a / c)^{n-1} \neq 0$ ignoring a factor of $c^{n-1}$. Note that $r^{2 n}=$ $(a / c)^{2 n(n-1)}=1$. Then we do another diagonal transformation of $D_{2}=\left[\begin{array}{ll}1 & 0 \\ 0 & r^{1 / 2}\end{array}\right]$ to get Pl-Holant $\left(\neq_{2} \mid\left(Z D_{1} D_{2}\right)^{-1} \mathcal{F},=_{2},\left(D_{2}^{-1}\right)^{\otimes 4 n}\left(=_{4 n}\right)\right)$. Notice that

$$
\left(D_{2}^{-1}\right)^{\otimes 4 n}(=4 n)=\left[1,0, \ldots, 0, r^{-2 n}\right]=(=4 n),
$$

as $r^{2 n}=1$ 
Hence we have $=2$ and $=_{4 n}$ on the right. With $\neq_{2}$ on the left, we get $=_{2}$ on the left and therefore equalities of all even arities on the right. Let $D=\left(D_{1} D_{2}\right)^{-1}$. Then we have the reduction chain:

$$
\begin{aligned}
\operatorname{Pl} \text {-Holant }(\mathcal{F}) & \geq_{T} \text { Pl-Holant }\left(\neq_{2} \mid D Z^{-1} \mathcal{F} \cup\left\{=_{2},=_{4 n}\right\}\right) \\
& \geq_{T} \text { Pl-Holant }\left(\neq_{2} \mid D Z^{-1} \mathcal{F} \cup \mathcal{E} \mathcal{Q}_{2}\right) \\
& \geq_{T} \text { Pl-Holant }\left(\mathcal{E} \mathcal{Q}_{2} \mid D Z^{-1} \mathcal{F}\right) .
\end{aligned}
$$

The last problem is $\operatorname{Pl}-\# \operatorname{CSP}^{2}\left(D Z^{-1} \mathcal{F}\right)$. Thus $\operatorname{Pl-\# CSP}{ }^{2}\left(D Z^{-1} \mathcal{F}\right) \quad \leq_{T}$ $\operatorname{Pl}-$ Holant $(\mathcal{F})$.

Finally, we strengthen Corollary 7.5, Lemma 7.7, Lemma 7.8, Lemma 7.10 and Lemma 7.11, including weighted equalities. We split the hardness and tractability cases. For a set $\mathcal{F}$ of signatures, denote by $\mathcal{F}_{n d}^{\geq 3}$ the set of non-degenerate signatures in $\mathcal{F}$ of arity at least 3 . Moreover denote by $\mathcal{F}^{*}$ the signature set that is the same as $\mathcal{F}$ but with each degenerate signature $[a, b]^{\otimes m}$ in $\mathcal{F}$ replaced by the unary signature $[a, b]$.

Notice that $\mathcal{F} \cap \mathscr{P}_{2}$ and $\mathcal{F}^{*} \cap \mathscr{P}_{2}$ agree on signatures of arity at least 2 , since signatures in $\mathscr{P}_{2}$ of arity at least 2 are non-degenerate. So $\mathcal{F} \cap \mathscr{P}_{2} \subseteq \mathcal{F}^{*} \cap \mathscr{P}_{2}$, and the only possible extra elements are some unary $[x, y]^{\text {'s }}$ from $[x, y]^{\otimes m} \in \mathcal{F}$ for some integer $m \geq 2$ and $[x, y]$ is not a multiple of $[1, \pm i]$. Equivalently the only possible extra elements are unary signatures of the form $Z[a, b]$ for $a b \neq 0$, i.e., not of the form a multiple of $Z[1,0]$ or $Z[0,1]$, when $Z^{-1} \mathcal{F}$ contains some degenerate signatures of the form $[a, b]^{\otimes m}$ for some integer $m \geq 2$ and $a b \neq 0$.

Lemma 7.15 Let $\mathcal{F}$ be a set of symmetric signatures. Let $\mathcal{F}_{n d}^{\geq 3}$ be the set of nondegenerate signatures in $\mathcal{F}$ of arity at least 3. Suppose $\mathcal{F}_{n d}^{\geq 3}$ contains some $f \in \mathscr{M}_{4}$ of arity $d \geq 3$. Moreover, suppose $\mathcal{F}_{n d}^{\geq 3} \cap \mathscr{P}_{2}$ is nonempty, and let $k$ be the greatest common divisor of the arities of signatures in $\mathcal{F}^{*} \cap \mathscr{P}_{2}$. If $k \leq 4$, then $\operatorname{Pl}-\operatorname{Holant}(\mathcal{F})$ is \#P-hard.

Proof We are given $f \in \mathscr{M}_{4}$, which is the union of $\mathscr{M}_{4}^{+}$and $\mathscr{M}_{4}^{-}$. We may assume that $f \in \mathscr{M}_{4}^{+}$, the other case is symmetric. Since $\mathcal{F}_{n d}^{\geq 3} \cap \mathscr{P}_{2}$ is nonempty, there exists $g \in \mathcal{F}_{n d}^{\geq 3} \cap \mathscr{P}_{2}$. By the definition of $\mathcal{F}_{n d}^{\geq 3}, g$ has arity $n \geq 3$. Under a $Z$ transformation,

$$
\operatorname{Pl-Holant}(\mathcal{F}) \equiv \operatorname{Pl-Holant}\left(\neq_{2} \mid \hat{g}, \operatorname{ExACTONE}_{d}, Z^{-1} \mathcal{F}\right),
$$

where $\hat{g}=\left(Z^{-1}\right)^{\otimes n} g$ has the form $[1,0, \ldots, 0, c]$ of arity $n$ for some $c \neq 0$ up to a nonzero factor, and EXACTONE $d$ comes from $f=Z^{\otimes d}$ EXACTONE $_{d}$. We further do a diagonal transformation $D=\left[\begin{array}{ll}1 & 0 \\ 0 & c^{1 / n}\end{array}\right]$ and get

$$
\operatorname{Pl}-\text { Holant }(\mathcal{F}) \equiv \operatorname{Pl} \text {-Holant }\left(\neq_{2} \mid={ }_{n}, \operatorname{ExACTONE}_{d},(Z D)^{-1} \mathcal{F}\right),
$$

where we ignore nonzero factors on $\neq_{2}$ and EXACTONE $_{d}$. 
The proof of this lemma is divided into two parts: We first prove the lemma when $\mathcal{F}^{*} \cap \mathscr{P}_{2} \neq \mathcal{F} \cap \mathscr{P}_{2}$. In this case, there exists some degenerate signature $(Z D[a, b])^{\otimes m} \in \mathcal{F}$ for some $a b \neq 0$ and $m \geq 1$. Since $\mathcal{F}^{*} \cap \mathscr{P}_{2}$ contains unary signatures, we have $k=1$, where $k$ is the gcd of arities defined in the statement of the lemma. We prove that in this case the problem Pl-Holant $(\mathcal{F})$ is \#P-hard. Indeed, we have

$$
\operatorname{Pl-Holant}\left(\neq_{2} \mid={ }_{n}, \operatorname{ExACTONE}_{d},[a, b]^{\otimes m}\right) \leq_{T} \operatorname{Pl-Holant}(\mathcal{F}),
$$

and we prove the former problem is \#P-hard.

We write $d=2+\delta$, with $\delta \geq 1$, and we perform the following operations.

1. Let $r=\operatorname{gcd}(n, \delta) \geq 1$. There exist positive integers $\ell_{1}$ and $\ell_{2}$ such that $r=\ell_{1} n-\ell_{2} \delta$. By Lemma 7.4 we can construct the signatures $=\ell_{1} n$ and $\operatorname{EXACTONE}_{2+\ell_{2} \delta}$, for all $\ell_{1}, \ell_{2} \geq 1$. By a self-loop on EXACTONE $2+\ell_{2} \delta$ via $\neq_{2}$ we get $[1,0]^{\otimes \ell_{2} \delta}$. Connect it to $=_{\ell_{1} n}$ via $\neq_{2}$ we get $[0,1]^{\otimes r}$ on right hand side (RHS) of Pl-Holant $\left(\neq_{2} \mid={ }_{n}\right.$, EXACTONE $\left._{d},[a, b]^{\otimes m}\right)$.

2. Let $s=\operatorname{gcd}(m, r) \geq 1$. There exist positive integers $\ell_{3}$ and $\ell_{4}$ such that $s=$ $\ell_{3} m-\ell_{4} r$. Take $\ell_{3}$ copies of $[a, b]^{\otimes m}$ and connect them to $\ell_{4}$ copies of $[0,1]^{\otimes r}$ via $\neq \neq_{2}$, we get a nonzero multiple of $[a, b]^{\otimes s}$ on RHS.

Note that $s|m, s| r$, and therefore $s \mid n$ and $s \mid \delta$.

3. Connect $r / s-1$ copies of $[a, b]^{\otimes s}$ to $[0,1]^{\otimes r}$ via $\neq 2$ we get a nonzero multiple of $[0,1]^{\otimes s}$ on RHS. (If $r=s$ then we already had $[0,1]^{\otimes s}$.)

4. Connect $\delta / s-1$ copies of $[0,1]^{\otimes s}$ to $\operatorname{EXACTONE}_{2+\delta}$ via $\neq_{2}$, we get EXACTONE$_{2+s}$ on RHS. (If $\delta=s$ then we already had EXACTONE $2+s$.)

5. Connect $n / s-1$ copies of $[a, b]^{\otimes s}$ to $=_{n}$ via $\neq 2$, we get a weighted EQUALITY function $\left[c_{1}, 0, \ldots, 0, c_{2}\right]$ of arity $s$, for some $c_{1} c_{2} \neq 0$. Under a diagonal transformation $D^{\prime}=\left[\begin{array}{ll}c_{2}^{1 / s} & 0 \\ 0 & c_{1}^{1 / s}\end{array}\right]$, it becomes $=_{s}$, up to a factor $c_{1} c_{2} \neq 0$.

The diagonal transformation $D^{\prime}$ does not change $\neq_{2}$ and EXACTONE $2+s$, up to a nonzero factor. And $[a, b]^{\otimes s}$ is transformed to another $\left[a^{\prime}, b^{\prime}\right]^{\otimes s}$, for some $a^{\prime} b^{\prime} \neq 0$.

6. Now we show that Pl-Holant $\left(\neq_{2} \mid={ }_{s}\right.$, EXACTONE $\left.2+s,\left[a^{\prime}, b^{\prime}\right]^{\otimes s}\right)$ is \#P-hard. We can move $={ }_{s}$ to the left hand side by connecting it to $s$ copies of $\neq_{2}$, thus

$$
\begin{aligned}
& \text { Pl-Holant }\left(\neq_{2},={ }_{s} \mid \operatorname{EXACTONE}_{2+s},\left[a^{\prime}, b^{\prime}\right]^{\otimes s}\right) \leq_{T} \\
& \text { Pl-Holant }\left(\neq_{2} \mid=_{s}, \text { EXACTONE }_{2+s},\left[a^{\prime}, b^{\prime}\right]^{\otimes s}\right) .
\end{aligned}
$$

Connecting $\left[a^{\prime}, b^{\prime}\right]^{\otimes s}$ to $\operatorname{EXACTONE}_{2+s}$ via $\neq 2$ gives us a binary signature $h=$ $\left[s a^{\prime}\left(b^{\prime}\right)^{s-1},\left(b^{\prime}\right)^{s}, 0\right]$, which is $\left[s a^{\prime}, b^{\prime}, 0\right]$ up to a nonzero factor. Hence

$$
\text { Pl-Holant }\left(=_{s} \mid h\right) \leq T \text { Pl-Holant }(\mathcal{F}) .
$$

Finally, Pl-Holant $\left(=_{s} \mid h\right)$ is \#P-hard by Theorem 2.24.

This completes the first part of the proof, and now for the second part we assume $\mathcal{F}^{*} \cap \mathscr{P}_{2}=\mathcal{F} \cap \mathscr{P}_{2}$.

By a weighted equality we mean a signature of the form $[a, 0, \ldots, 0, b]$ of some arity $\geq 1$, where $a b \neq 0$. Recall that $\mathscr{P}_{2}$ consists of the $Z$ transformation of all weighted equalities (see Lemma 3.2). Weighted equalities are transformed to 
weighted equalities under the diagonal transformation $D$. Let $\mathcal{G}$ be the set of weighted equalities in $(Z D)^{-1} \mathcal{F}$. In other words, $\mathcal{G}=(Z D)^{-1}\left(\mathcal{F} \cap \mathscr{P}_{2}\right)$ as $(Z D)^{-1} \mathscr{P}_{2}$ contains all weighted equalities. Moreover, up to a nonzero factor, $\left(=_{n}\right) \in \mathcal{G}$, as the signature $g=(Z D)^{\otimes n}\left(={ }_{n}\right) \in \mathcal{F}_{n d}^{\geq 3} \cap \mathscr{P}_{2} \subseteq \mathcal{F} \cap \mathscr{P}_{2}$.

Pick any $g_{1}, g_{2} \in \mathcal{G}$ of arities $\ell_{1}$ and $\ell_{2}$. Let $r=\operatorname{gcd}\left(\ell_{1}, \ell_{2}\right)$. Let $t_{1}, t_{2}$ be two positive integers such that $t_{1} \ell_{1}-t_{2} \ell_{2}=r$. Then connecting $t_{1}$ copies of $g_{1}$ and $t_{2}$ copies of $g_{2}$ via $\neq 2$ in a bipartite and planar way, we get a weighted equality signature of arity $r$.

Applying the same argument repeatedly, we can construct a weighted equality $h$ of arity $k$. We can choose a diagonal transformation $D_{1}$ of rank 2 that transforms it to $=_{k}$, that is,

$$
\begin{aligned}
\operatorname{Pl} \text {-Holant }(\mathcal{F}) & \geq_{T} \text { Pl-Holant }\left(\neq_{2} \mid \mathcal{G}, \operatorname{ExACTONE}_{d}\right) \\
& \geq_{T} \text { Pl-Holant }\left(\neq_{2} \mid h, \operatorname{ExACTONE}_{d}, \mathcal{G}\right) \\
& \geq_{T} \text { Pl-Holant }\left(\left(\neq_{2}\right) D_{1}^{\otimes 2} \mid=_{k},\left(D_{1}^{-1}\right)^{\otimes d} \operatorname{ExACTONE}_{d}, D_{1}^{-1} \mathcal{G}\right) \\
& \geq_{T} \text { Pl-Holant }\left(\neq_{2} \mid=_{k}, \operatorname{ExACTONE}_{d}, D_{1}^{-1} \mathcal{G}\right),
\end{aligned}
$$

where in the last line we ignored nonzero factors of ExACTONE $d$ and $\neq_{2}$. If $k=3$ or 4 , then the hardness follows from Corollary 7.5.

If $k=1$ or 2 , then on the right hand side we have $=_{k}$, which is $=_{1}$ or $=_{2}$, and a weighted equality $\left(D_{1}^{-1}\right)^{\otimes n}\left(=_{n}\right) \in D_{1}^{-1} \mathcal{G}$, where $n \geq 3$. Call it $\hat{g}^{\prime}$. We move the $=_{k}$ to the left hand side via $\neq_{2}$. Then we connect zero or more copies of this $=_{k}$, which is $=_{1}$ or $=_{2}$, to $\hat{g}^{\prime}$ such that its arity is 3 or 4 . (It is possible that $n=3$ or 4 to begin with, and if so we do nothing.) We are done by yet another diagonal transformation and Corollary 7.5.

Lemma 7.16 Let $\mathcal{F}$ be a set of symmetric signatures. Suppose $\mathcal{F} \subseteq Z \mathscr{P} \cup \mathscr{M}_{4}^{\sigma}$ for some $\sigma \in\{+,-\}$ and the greatest common divisor of the arities of all signatures in $\mathcal{F}^{*} \cap \mathscr{P}_{2}$ is $k \geq 5$. Then $\mathrm{Pl}-$ Holant $(\mathcal{F})$ can be computed in polynomial time.

Proof We may assume that $\sigma=+$ and the case of $\sigma=-$ is similar. We do a $Z$ transformation on Pl-Holant $(\mathcal{F})$, and get a problem of Pl-Holant $\left(\neq_{2} \mid Z^{-1} \mathcal{F}\right)$.

As stated after Definition 2.6, symmetric signatures in $\mathscr{P}$ are either degenerate, or binary Disequality $\neq 2$, or $[a, 0, \ldots, 0, b]$ for some $a, b \in \mathbb{C}$. There is a 1-1 correspondence between degenerate signatures in $\mathcal{F}$ and degenerate signatures in $Z^{-1} \mathcal{F}$, and every degenerate signature in $\mathcal{F}$ can be written as $(Z[a, b])^{\otimes m}$ for some $m \geq 1$ and $a, b \in \mathbb{C}$. If $a b \neq 0$, then $Z[a, b] \in \mathcal{F}^{*} \cap \mathscr{P}_{2}$. This contradicts $k \geq 5$. Thus either $a=0$ or $b=0$, and thus up to a scalar factor, all degenerate signatures in $\mathcal{F}$ are of the form $(Z[1,0])^{\otimes m}$ or $(Z[0,1])^{\otimes m}$, if any.

We next consider what nondegenerate signatures can there be in $Z^{-1} \mathcal{F}$. As $\mathcal{F} \subseteq$ $Z \mathscr{P} \cup \mathscr{M}_{4}^{+}$, we have $Z^{-1} \mathcal{F} \subseteq \mathscr{P} \cup Z^{-1} \mathscr{M}_{4}^{+}$. Nondegenerate symmetric signatures in the set $\mathscr{P}$ consist of weighted equalities and $\left(\neq_{2}\right)$ (which is also ExACTONE 2 ), and signatures in $Z^{-1} \mathscr{M}_{4}^{+}$are just the EXACTONE $d$ signatures of arity $d \geq 1$. The 
EXACTONE$_{1}$ and EXACTONE 2 signatures are $[0,1]$ and $(\neq 2)$ respectively; those with $d \geq 3$ are in the set $\mathcal{E} \mathcal{O}$. The nondegenerate weighted equalities in $Z^{-1} \mathcal{F}$ are in the set $Z^{-1}\left(\mathcal{F}^{*} \cap \mathscr{P}_{2}\right)$, thus have arity a multiple of $k$, where $k \geq 5$.

In the bipartite setting of Pl-Holant $\left(\neq_{2} \mid Z^{-1} \mathcal{F}\right)$, if given $=_{n}$ on the right hand side, we can realize $=\ell n$ for any integer $\ell \geq 1$ as an $E_{n}$-block on the right. By Lemma 7.7 and Lemma 7.10, the problem Pl-Holant $\left(\neq_{2} \mid \mathcal{E} \mathcal{Q}_{n}, \mathcal{E} \mathcal{O}, \neq_{2},[1,0],[0,1]\right)$ is tractable for any $n \geq 5$, where $\mathcal{E} \mathcal{Q}_{n}$ denotes the set of all equalities of arity $\ell n$ for all integers $\ell \geq 1$. Pl-Holant $(\mathcal{F})$ is an instance of Pl-Holant $\left(\neq_{2} \mid \mathcal{E} \mathcal{Q}_{k}, \mathcal{E} \mathcal{O}, \neq_{2},[1,0],[0,1]\right)$ except for the weights on the equalities. However this weighted version is tractable by Lemma 7.8 and Lemma 7.11.

Let $\mathcal{G}=\left\{=_{k} \mid k \in S\right\}$ be a set of EQUALITY signatures, where $S$ is a set of positive integers containing at least one $r \geq 3$. Moreover let $\mathcal{E} \mathcal{O}^{+}:=\left\{\right.$EXACTONE $_{d} \mid$ $\left.d \in \mathbb{Z}^{+}\right\}=\mathcal{E} \mathcal{O} \cup\{\neq 2,[0,1]\}$. Then Pl-Holant $\left(\mathcal{G} \mid \mathcal{E} \mathcal{O}^{+}\right)$is the problem of counting perfect matchings over hypergraphs with planar incidence graphs, where the hyperedge sizes are prescribed by $S$. In the incidence graph, vertices assigned signatures in $\mathcal{G}$ on the left represent hyperedges, and vertices assigned signatures in $\mathcal{E O}^{+}$on the right represent vertices of the hypergraph. Let $t=\operatorname{gcd}(S)$. It is stated in the introduction that this problem is tractable if $t \geq 5$ and \#P-hard if $t \leq 4$. The tractability when $t \geq 5$ follows from Lemma 7.7 and 7.10, since we can reduce Pl-Holant $\left(\mathcal{G} \mid \mathcal{E} \mathcal{O}^{+}\right)$ to Pl-Holant $\left(\neq_{2} \mid=_{t}, \mathcal{E O}, \neq_{2},[0,1]\right)$. The reduction goes as follows. With $\neq_{2}$ on the left hand side and $=_{t}$ on the right hand side, we can construct all $E_{t}$-blocks and hence all of $\mathcal{E} \mathcal{Q}_{t}$ on the right. Note that $\mathcal{G} \subseteq \mathcal{E} \mathcal{Q}_{t}$. Then we move all signatures in $\mathcal{G}$ to the left via $\neq_{2}$.

The \#P-hardness of Pl-Holant $\left(\mathcal{G} \mid \mathcal{E} \mathcal{O}^{+}\right)$for $t \leq 4$ follows from Corollary 7.5. We give a reduction to $\mathrm{Pl}$-Holant $\left(\mathcal{G} \mid \mathcal{E} \mathcal{O}^{+}\right)$. We construct $\neq_{2}$ on the left using the gadget pictured in Fig. 7a with $\left(=_{r}\right) \in \mathcal{G}$ on the left side assigned to circle vertices and $\neq_{2}$ on the right side assigned to square vertices. Then we move $\mathcal{G}$ to the right side via $\neq_{2}$ on the right side. We construct $={ }_{t}$ on the right side in the same Euclidean process using $\mathcal{G}$ of the right side and $\neq_{2}$ of the left side. This gives us a reduction from Pl-Holant $\left(\neq_{2} \mid={ }_{t}, \mathcal{E O}\right)$, which is \#P-hard by Corollary 7.5 if $t=3$, 4. Otherwise $t=1,2$. Recall that $\left(=_{r}\right) \in \mathcal{G}$ for some $r \geq 3$. We use $={ }_{t}$ to reduce the arity of $=_{r}$ to 3 or 4 , if necessary. Again we are done by Corollary 7.5.

If there is no hyperedge of size $\geq 3$ in $\operatorname{Pl-Holant}\left(\mathcal{G} \mid \mathcal{E} \mathcal{O}^{+}\right)$, then for $S=\{1\}$, the problem is tractable trivially, and for $S=\{2\}$, the problem is tractable by Kasteleyn's algorithm. The problem is \#P-hard if $S=\{1,2\}$, because we can realize not-necessarily perfect matchings $[1,1,0, \ldots, 0]$ (ATMOSTONE $d$ ) by connecting $(=1)=[1,1]$ to EXACTONE $_{d+1}$. In summary, we have the following theorem.

Theorem 7.17 Let $S$ be a set of positive integers. Let $\mathcal{G}=\left\{=_{k} \mid k \in S\right\}$. The problem Pl-Holant $\left(\mathcal{G} \mid \mathcal{E O}^{+}\right)$counts perfect matchings over hypergraphs with planar incidence graphs, where the hyperedge sizes are prescribed by the set $S$. Let $t=\operatorname{gcd}(S)$. If $t \geq 5$ or $S=\{1\}$ or $\{2\}$, then the problem is computable in polynomial time. Otherwise $t \leq 4, S \neq\{1\}$ and $S \neq\{2\}$, and the problem is \#P-hard. 


\section{Full Dichotomy of PI-Holant $(\mathcal{F})$}

We are finally ready to prove our main dichotomy theorem. Recall that for a set $\mathcal{F}$ of signatures, $\mathcal{F}_{n d}^{\geq 3}$ denotes the set of non-degenerate signatures in $\mathcal{F}$ of arity at least 3 , and $\mathcal{F}^{*}$ denotes $\mathcal{F}$ with all degenerate signatures $[a, b]^{\otimes m}$ replaced by unary $[a, b]$.

Theorem 8.1 Let $\mathcal{F}$ be any set of symmetric, complex-valued signatures in Boolean variables. Then $\mathrm{Pl}-\mathrm{Holant}(\mathcal{F})$ is computable in polynomial time if $\mathcal{F}$ satisfies one of the following conditions:

1. All non-degenerate signatures in $\mathcal{F}$ are of arity at most 2 ;

2. $\mathcal{F}$ is $\mathscr{A}$-transformable;

3. $\mathcal{F}$ is $\mathscr{P}$-transformable;

4. $\mathcal{F} \subseteq \mathscr{V}^{\sigma} \cup\left\{f \in \mathscr{R}_{2}^{\sigma} \mid \operatorname{arity}(f)=2\right\}$ for some $\sigma \in\{+,-\}$;

5. All non-degenerate signatures in $\mathcal{F}$ are in $\mathscr{R}_{2}^{\sigma}$ for some $\sigma \in\{+,-\}$.

6. $\mathcal{F}$ is $\mathscr{M}$-transformable;

7. $\mathcal{F} \subseteq Z \mathscr{P} \cup \mathscr{M}_{4}^{\sigma}$ for some $\sigma \in\{+,-\}$, and the greatest common divisor of the arities of the signatures in $\mathcal{F}^{*} \cap \mathscr{P}_{2}$ is at least 5 .

If $\mathcal{F}$ does not satisfy any of these conditions, then Pl-Holant $(\mathcal{F})$ is \#P-hard. Furthermore, if $\mathcal{F}$ satisfies condition 1, 2, 3, 4, or 5, then $\operatorname{Holant}(\mathcal{F})$ is computable in polynomial time without assuming planarity; otherwise (non-planar) $\operatorname{Holant}(\mathcal{F})$ is \#P-hard.

Proof We may assume that $\mathcal{F}$ contains no identically 0 signatures. We note that removing any identically 0 signature from a set does not affect its complexity, being either tractable or \#P-hard, and it does not affect the set $\mathcal{F}$ satisfying any of the listed conditions in Case 1 to 7.

If all non-degenerate signatures in $\mathcal{F}$ are of arity at most 2 , then the problem is tractable case 1. Otherwise, there is a non-degenerate signature $f \in \mathcal{F}$ of arity at least 3. By Theorem 6.1, Pl-Holant $(\mathcal{F})$ is \#P-hard unless $f \in \mathscr{P}_{1} \cup \mathscr{M}_{2} \cup \mathscr{A}_{3} \cup \mathscr{M}_{3} \cup$ $\mathscr{M}_{4}$ or $f \in \mathscr{V}$. If $f \in \mathscr{P}_{1}, f \in \mathscr{M}_{2} \backslash \mathscr{P}_{2}, f \in \mathscr{A}_{3}$, or $f \in \mathscr{M}_{3}$, then we are done by Corollary 5.4, Lemma 5.8, Corollary 5.6, or Lemma 5.10 respectively. Therefore, we assume that none of these is the case. This implies that $\mathcal{F}_{n d}^{\geq 3}$ is nonempty and that each of its signatures is in $\mathscr{P}_{2}$ or in $\mathscr{M}_{4}$ or vanishing. That is,

$$
\emptyset \neq \mathcal{F}_{n d}^{\geq 3} \subseteq \mathscr{P}_{2} \cup \mathscr{M}_{4} \cup \mathscr{V} .
$$

In fact, as stated after Lemma 3.10 , all signatures in $\mathscr{M}_{4}$ of arity at least 3 already belong to $\mathscr{V}$. We keep $\mathscr{M}_{4}$ in the union because $\mathscr{M}_{4}$ is treated separately than $\mathscr{V} \backslash \mathscr{M}_{4}$ in the following proof.

Suppose there exists some $f \in \mathcal{F}_{n d}^{\geq 3}$ that is in $\mathscr{V} \backslash \mathscr{M}_{4}$. We assume $f \in \mathscr{V}^{+}$since the other case $\mathscr{V}^{-}$is similar. In this case, we show that $\mathrm{Pl}-\operatorname{Holant}(\mathcal{F})$ is \#P-hard, unless $\mathcal{F}$ is in Case 4 or Case 5 . Assume that Pl-Holant $(\mathcal{F})$ is not \#P-hard. ${ }^{2}$ We will

\footnotetext{
${ }^{2}$ Formally, this statement has meaning regardless whether \#P collapses to $\mathrm{P}$. We prove Theorem 8.1 as a logical implication: If $\mathrm{Pl}$-Holant $(\mathcal{F})$ is not \#P-hard then $\mathcal{F}$ belongs to one of the seven cases, and in each case the problem is P-time computable. Of course, the dichotomy is only meaningful if \#P does not collapse to P. But the validity of the dichotomy theorem is proved without making this explicit assumption.
} 
discuss non-degenerate signatures of arity $\geq 3$, of arity 2 , and degenerate signatures separately.

1. For any $g \in \mathcal{F}_{n d}^{\geq 3}$, since signatures in $\mathscr{M}_{4}$ of arity at least 3 already belong to $\mathscr{V}$, we have $g \in \mathscr{P}_{2} \cup \mathscr{V}$. If $g \in \mathscr{P}_{2}$, then Pl-Holant $(f, g)$ is \#P-hard by Lemma 4.7. If $g \in \mathscr{V}^{-}$, then Pl-Holant $(f, g)$ is \#P-hard by Lemma 4.5 as $f \notin \mathscr{M}_{4}$. Therefore we may assume $g \in \mathscr{V}^{+}$.

2. For any non-degenerate binary signature $h \in \mathcal{F}$, it must be that $h \in \mathscr{R}_{2}^{+}$as otherwise Pl-Holant $(f, h)$ is \#P-hard by Lemma 4.3.

3. If $\mathrm{rd}^{+}(g)=1$ for all $g \in \mathcal{F}_{n d}^{\geq 3}$, then $\mathcal{F}_{n d}^{\geq 3} \subseteq \mathscr{R}_{2}^{+}$by Lemma 2.19. Together with the fact just proved that all non-degenerate binary in $\mathcal{F}$ are in $\mathscr{R}_{2}^{+}$, Case 5 is satisfied.

Otherwise there exists $g \in \mathcal{F}_{n d}^{\geq 3}$ such that $\mathrm{rd}^{+}(g) \geq 2$. By the first item above, $g \in \mathscr{V}^{+}$. If $\mathcal{F}$ contains any degenerate signature $v=u^{\otimes m}$ for $m \geq 1$ and some unary $u$ that is not a multiple of $[1, i]$, then by Lemma $4.1, \operatorname{Pl}-\operatorname{Holant}(g, v)$ is \#P-hard. Hence all degenerate signatures are multiples of tensor powers of $[1, i]$, which are in $\mathscr{V}^{+}$. This implies that $\mathcal{F}$ is in Case 4.

Now we have that $\emptyset \neq \mathcal{F}_{n d}^{\geq 3} \subseteq \mathscr{P}_{2} \cup \mathscr{M}_{4}$. We handle this in three cases.

1. Suppose $\mathcal{F}_{n d}^{\geq 3} \subseteq \mathscr{M}_{4}$. First suppose $\mathcal{F}_{n d}^{\geq 3} \subseteq \mathscr{M}_{4}^{\sigma}$ for some $\sigma \in\{+,-\}$. Assume $\sigma=+$ as $\sigma=-$ is similar. Then $\mathcal{F}_{n d}^{\geq 3} \subseteq \mathscr{R}_{2}^{+}$by Lemmas 3.10 and 2.19. If all non-degenerate binary signatures are in $\mathscr{R}_{2}^{+}$as well, then this is Case 5 and tractable. Otherwise let $h$ be a non-degenerate binary signature in $\mathcal{F}$ that is not in $\mathscr{R}_{2}^{+}$. We apply Lemma 4.4, and Pl-Holant $(\mathcal{F})$ is \#P-hard unless $h=Z^{\otimes 2}[a, 0,1]$ up to a nonzero factor, where $a \neq 0$. In this case we apply a $Z$ transformation, and get Pl-Holant $\left(\neq 2 \mid[a, 0,1], Z^{-1} \mathcal{F}\right)$. Then we do a diagonal transformation $D=\left[\begin{array}{lr}a^{1 / 2} & 0 \\ 0 & 1\end{array}\right]$. Note that this only changes $\neq_{2}$ on the left hand side to a nonzero multiple of $\neq_{2}$. Hence we have the reduction chain:

$$
\begin{aligned}
\operatorname{Pl} \text {-Holant }(\mathcal{F}) & \equiv \text { Pl-Holant }\left(\neq_{2} \mid[a, 0,1], Z^{-1} \mathcal{F}\right) \\
& \equiv \text { Pl-Holant }\left(\neq_{2} \mid[1,0,1], D^{-1} Z^{-1} \mathcal{F}\right) \\
& \geq_{T} \text { Pl-Holant }\left(D^{-1} Z^{-1} \mathcal{F}\right),
\end{aligned}
$$

where the last reduction is by a chain of $\left(\neq_{2}\right),\left(=_{2}\right)=[1,0,1]$ and $\left(\neq_{2}\right)$, which gives $(=2)$ on the LHS. Notice that $D^{-1} Z^{-1} \mathcal{F}$ contains EXACTONE $k$ with $k \geq$ 3. To see that, recall that we have $\emptyset \neq \mathcal{F}_{n d}^{\geq 3} \subseteq \mathscr{M}_{4}^{+}$. Signatures in $\mathscr{M}_{4}^{+}$have the form $Z[0,1,0, \ldots, 0]$ up to a nonzero scalar. A nonsingular diagonal $D$ does not change EXACTONE $k$ except for a nonzero scalar. This signature EXACTONE $_{k}$ is in $\mathscr{M}_{3}$ with orthogonal transformation $I_{2}$. Then by Lemma 5.10, Pl-Holant $(\mathcal{F})$ is \#P-hard unless $D^{-1} Z^{-1} \mathcal{F} \subseteq I_{2} \mathscr{M}=\mathscr{M}$, i.e., $\mathcal{F} \subseteq Z D \mathscr{M}=Z \mathscr{M}$. The case $\mathcal{F} \subseteq Z \mathscr{M}$ implies that $\mathcal{F}$ is $\mathscr{M}$-transformable via $Z$, and we are in tractable Case 6.

Otherwise $\mathcal{F}_{n d}^{\geq 3}$ contains both $f \in \mathscr{M}_{4}^{+}$and $g \in \mathscr{M}_{4}^{-}$. Similarly as above, by Lemma 4.4, if Pl-Holant $(\mathcal{F})$ is not \#P-hard, then any non-degenerate binary 
signature in $\mathcal{F}$ has to be either in $\mathscr{R}_{2}^{+} \cap \mathscr{R}_{2}^{-}$, or a nonzero constant multiple of $Z^{\otimes 2}[a, 0,1]$ where $a \neq 0$. For non-degenerate binary signatures, belonging to $\mathscr{R}_{2}^{+} \cap \mathscr{R}_{2}^{-}$is just $\left\{Z^{\otimes 2}(\neq 2)\right\}$ up to a nonzero constant (cf. Lemma 2.19). Moreover, by Lemma 4.6, Pl-Holant $(\mathcal{F})$ is \#P-hard, unless all degenerate signatures in $\mathcal{F}$ are of the form $[1, \pm i]^{\otimes m}$. Note that $[1, i]=Z[1,0]$ and $[1,-i]=Z[0,1]$. When this is the case, $\mathcal{F}$ is $\mathscr{M}$-transformable via $Z$, and we are in tractable Case 6.

2. Suppose $\mathcal{F}_{n d}^{\geq 3} \subseteq \mathscr{P}_{2}$. If $\mathcal{F}$ contains a non-degenerate binary signature $h$, then we apply Lemma 7.14 and Pl-Holant $(\mathcal{F})$ is \#P-hard unless $h \in Z \mathscr{P}$, or $\mathrm{Pl}-\# \mathrm{CSP}^{2}\left(D Z^{-1} \mathcal{F}\right) \leq_{T}$ Pl-Holant $(\mathcal{F})$ for some diagonal transformation $D$. If it is the latter case, then by Theorem 5.1, either Pl-Holant $(\mathcal{F})$ is \#P-hard, or $D Z^{-1} \mathcal{F}$ is a subset of $T \mathscr{A}, \mathscr{P}$, or $T\left[\begin{array}{ll}1 & 1 \\ 1 & -1\end{array}\right] \mathscr{M}$, for some diagonal matrix $T$. We claim that in any of these cases Pl-Holant $(\mathcal{F})$ is tractable. In fact,

(a) if $D Z^{-1} \mathcal{F} \subseteq T \mathscr{A}$, then $\mathcal{F}$ is $\mathscr{A}$-transformable as $\mathcal{F} \subseteq Z D^{-1} T \mathscr{A}$ and $[1,0,1]$ (as a row vector) is transformed into $[1,0,1]\left(Z D^{-1} T\right)^{\otimes 2}$, which is $[0,1,0] \in \mathscr{A}$ up to a nonzero constant;

(b) if $D Z^{-1} \mathcal{F} \subseteq \mathscr{P}$, then $\mathcal{F}$ is $\mathscr{P}$-transformable as $\mathcal{F} \subseteq Z D^{-1} \mathscr{P}$ and $[1,0,1]\left(Z D^{-1}\right)^{\otimes 2}$ is $[0,1,0] \in \mathscr{P}$ up to a nonzero constant;

(c) if $D Z^{-1} \mathcal{F} \subseteq T\left[\begin{array}{ll}1 & 1 \\ 1 & -1\end{array}\right] \mathscr{M}$, then $\mathcal{F}$ is $\mathscr{M}$-transformable as $\mathcal{F} \subseteq Z D^{-1} T\left[\begin{array}{ll}1 & 1 \\ 1 & -1\end{array}\right] \mathscr{M}$ and $[1,0,1]$ is transformed to $[1,0,1]\left(Z D^{-1} T\left[\begin{array}{ll}1 & 1 \\ 1 & -1\end{array}\right]\right)^{\otimes 2}$, which is $[1,0,-1] \in \mathscr{M}$ up to a nonzero constant.

Hence we may assume that every non-degenerate binary in $\mathcal{F}$ is in $Z \mathscr{P}$. Notice that degenerate signatures are always in $\mathscr{P}$ under any transformation. Also $\mathcal{F}_{n d}^{\geq 3}$ is a subset of $Z \mathscr{P}$ because $\mathcal{F}_{n d}^{\geq 3} \subseteq \mathscr{P}_{2}$ and $\mathscr{P}_{2}$ is just weighted equalities under $Z$-transformation. This implies that $\mathcal{F}$ is $\mathscr{P}$-transformable under the $Z$ transformation. Hence we are in Case 3.

3. Finally, suppose neither of the above is the case. Then there are $f, g \in \mathcal{F}_{n d}^{\geq 3}$ with $f \in \mathscr{M}_{4}$ and $g \in \mathscr{P}_{2}$. If $\mathcal{F}_{n d}^{\geq 3}$ contains signatures from both $\mathscr{M}_{4}^{+}$and $\mathscr{M}_{4}^{-}$, then Pl-Holant $(\mathcal{F})$ is \#P-hard by Lemma 7.13. Otherwise $\mathcal{F}_{n d}^{\geq 3} \cap \mathscr{M}_{4} \subseteq \mathscr{M}_{4}^{+}$or $\mathscr{M}_{4}^{-}$. Let $\mathcal{G}=\mathcal{F}^{*} \cap \mathscr{P}_{2}$, and let $d$ be the gcd of the arities of the signatures in $\mathcal{G}$. Then $\mathcal{G}$ contains at least one non-degenerate signature $g$ of arity $\geq 3$. If $d \leq 4$, then Pl-Holant $(\mathcal{F})$ is \#P-hard by Lemma 7.15. Otherwise $d \geq 5$. If $\mathcal{F}$ contains a non-degenerate binary signature $h$, then we apply Lemma 7.14 and by a similar analysis as in the case of " $\mathcal{F}_{n d}^{\geq 3} \subseteq \mathscr{P}_{2}$ " above, we are done unless every such $h$ is in $Z \mathscr{P}$. Ignoring a nonzero factor, this implies that either $h=Z^{\otimes 2}[1,0, a]$ where $a \neq 0$ or $h=Z^{\otimes 2}\left(\neq_{2}\right)$. If $h=Z^{\otimes 2}[1,0, a]$, then $h \in \mathcal{F}^{*} \cap \mathscr{P}_{2}$, and this contradicts $d \geq 5$. Hence $h=Z^{\otimes 2}\left(\neq_{2}\right)$. If there is any degenerate $v=(Z[a, b])^{\otimes m}$ in $\mathcal{F}$ with $a b \neq 0$, then $Z[a, b] \in \mathcal{F}^{*} \cap \mathscr{P}_{2}$ and this also contradicts $d \geq 5$. 
In summary, Pl-Holant $(\mathcal{F})$ is \#P-hard unless $\mathcal{F}$ satisfies all five properties: (1) $\mathcal{F}_{n d}^{\geq 3} \subseteq \mathscr{P}_{2} \cup \mathscr{M}_{4},(2) \mathcal{F}_{n d}^{\geq 3} \cap \mathscr{M}_{4} \subseteq \mathscr{M}_{4}^{\sigma}$ for some $\sigma \in\{+,-\}$, (3) the greatest common divisor of the arities of the signatures in $\mathcal{F}^{*} \cap \mathscr{P}_{2}$ is at least 5, (4) every non-degenerate binary in $\mathcal{F}$ is of the form $Z^{\otimes 2}\left(\neq_{2}\right)$, and (5) every degenerate signature in $\mathcal{F}$ is of the form $(Z[1,0])^{\otimes m}$ or $(Z[0,1])^{\otimes m}$. Notice that $\mathscr{P}_{2}$, $Z^{\otimes 2}\left(\neq_{2}\right),(Z[1,0])^{\otimes m}$, and $(Z[0,1])^{\otimes m}$ are all in $Z \mathscr{P}$. Hence the exceptional case implies that $\mathcal{F} \subseteq Z \mathscr{P} \cup \mathscr{M}_{4}^{\sigma}$ for some $\sigma \in\{+,-\}$ and the greatest common divisor of the arities of the signatures in $\mathcal{F}^{*} \cap \mathscr{P}_{2}$ is at least 5. This is tractable Case 7.

The tractability of Holant $(\mathcal{F})$ in Case 1, Case 2, Case 3, Case 4, and Case 5 follows from the Holant dichotomy Theorem 2.21, which also implies that (the non-planar) Holant $(\mathcal{F})$ is \#P-hard otherwise. The tractability of Pl-Holant $(\mathcal{F})$ in Case 6 follows from Theorem 2.7. The tractability of Pl-Holant $(\mathcal{F})$ in Case 7 follows from Lemma 7.16. This completes the proof.

\section{Part II Planar \#CSP² Dichotomy}

In Part II of this paper, we prove Theorem 9.2, which is the complexity dichotomy theorem of $\mathrm{Pl}-\# \mathrm{CSP}^{2}(\mathcal{F})$, where $\mathcal{F}$ is a set of complex-valued symmetric signatures on Boolean variables. After we define some relevant notions, we give an outline of the proof of Theorem 9.2. Throughout Part II, we denote by $\alpha$ (respectively $\rho$ ) any quantity that satisfies $\alpha^{4}=-1$ (respectively $\rho^{4}=1$ ) and $\epsilon= \pm 1$.

\section{Preliminaries}

We will first define some tractable families of signatures that are expressible under a holographic transformation, specific to the $\mathrm{Pl}-\# \mathrm{CSP}^{2}$ framework.

Definition 9.1 Let $\mathcal{T}_{k}=\left\{\left[\begin{array}{ll}1 & 0 \\ 0 & \theta\end{array}\right] \mid \theta^{k}=1\right\}$ be a set of diagonal matrices of order dividing $k$ and $\mathscr{T}_{k}=\mathcal{T}_{2 k} \backslash \mathcal{T}_{k}=\left\{\left[\begin{array}{ll}1 & 0 \\ 0 & \theta\end{array}\right] \mid \theta^{k}=-1\right\}$. Let $\mathscr{A}^{\dagger}=\mathscr{T}_{4} \mathscr{A}$ and $\widehat{\mathscr{M}^{\dagger}}=$ $\mathscr{T}_{2} \widehat{\mathscr{M}}$ be the sets of signatures transformed by $\mathscr{T}_{4}$ from the Affine family $\mathscr{A}$ and transformed by $\mathscr{T}_{2}$ from $\widehat{\mathscr{M}}$, respectively, where for a class of signatures $\mathscr{C}$, we denote

Let

$$
\mathscr{T}_{k} \mathscr{C}=\left\{T^{\otimes \operatorname{arity}(f)} f \mid T \in \mathscr{T}_{k} \text { and } f \in \mathscr{C}\right\}
$$

$$
\tilde{\mathscr{A}}=\mathscr{A} \cup \mathscr{A}^{\dagger} \quad \text { and } \quad \widetilde{\mathscr{M}}=\widehat{\mathscr{M}} \cup \widehat{\mathscr{M}}^{\dagger}
$$

be the $\mathscr{A}$-transformable and $\mathscr{M}$-transformable signatures for Pl-\#CSP${ }^{2}$.

Recall that $\widehat{\mathscr{M}}=H \mathscr{M}$ is the set of Matchgate signatures $\mathscr{M}$ transformed by the Hadamard basis $H=\left[\begin{array}{ll}1 & 1 \\ 1 & -1\end{array}\right]$. Note that $\mathscr{A}$ is unchanged under the transformation 
by $H$, and thus there is no need to define $\widehat{\mathscr{A}}$. Also note that $\mathscr{P}$ is unchanged under any diagonal matrix. Thus there is no need to define $\mathscr{P}^{\dagger}$. For $T=\left[\begin{array}{ll}1 & 0 \\ 0 & \rho\end{array}\right] \in \mathcal{T}_{4}$ with $\rho^{4}=1, T \mathscr{A}=\mathscr{A}$. Thus $\tilde{\mathscr{A}}$ consists of $\mathscr{A}$ under transformations by all $T=\left[\begin{array}{ll}1 & 0 \\ 0 & \theta\end{array}\right] \in \mathcal{T}_{8}$. For such $T$, we have $\left(={ }_{2 n}\right) T^{\otimes 2 n} \in \mathscr{A}$. Hence $\tilde{\mathscr{A}}$ is $\mathscr{A}$ transformable for Pl-\#CSP ${ }^{2}$. Similarly, for $T=\left[\begin{array}{ll}1 & 0 \\ 0 & \pm 1\end{array}\right], T H=\left[\begin{array}{ll}1 & 1 \\ \pm 1 & \mp 1\end{array}\right]=$ either $H$ or $H\left[\begin{array}{ll}0 & 1 \\ 1 & 0\end{array}\right]$, and $\left[\begin{array}{ll}0 & 1 \\ 1 & 0\end{array}\right] \mathscr{M}=\mathscr{M}$. Thus $T \widehat{\mathscr{M}}=\widehat{\mathscr{M}}$, and $\widetilde{\mathscr{M}}$ is $\mathscr{M}$ transformed under $T H$ for all $T \in \mathcal{T}_{4}$. Also note that for all such $T$, we have $(=2 n)(T H)^{\otimes 2 n} \in \mathscr{M}$. Hence $\widetilde{\mathscr{M}}$ is $\mathscr{M}$-transformable for Pl-\#CSP${ }^{2}$.

In the proof of No-Mixing of different tractable sets in later sections, because of a particular order in which we carry out the proof, to make an overall logical structure more apparent we introduce the following notations

$$
S_{1}=\widehat{\mathscr{M}}, \quad S_{2}=\widehat{\mathscr{M}}^{\dagger}, \quad S_{3}=\mathscr{A}^{\dagger}, \quad S_{4}=\mathscr{A}, \quad \text { and } \quad S_{5}=\mathscr{P} .
$$

We will prove the following Main Theorem of Part II. It is not hard to see that this is a rephrase of Theorem 5.1 from Part I. It follows from Theorem 11.13, Theorem 16.5 and Theorem 15.4, which will be shown in later sections. It follows from the definition of $\mathscr{P}$-transformability, $\mathscr{A}$-transformability and $\mathscr{M}$-transformability that if $\mathcal{F} \subseteq S_{k}$ for any $1 \leq k \leq 5$, then $\mathrm{Pl}_{-} \# \mathrm{CSP}^{2}(\mathcal{F})$ is tractable.

Theorem 9.2 For any set of complex-valued symmetric signatures $\mathcal{F}$ on Boolean variables, if $\mathcal{F} \subseteq \mathscr{P}$, or $\mathscr{A}$, or $\mathscr{A}^{\dagger}$, or $\widehat{\mathscr{M}}$, or $\widehat{\mathscr{M}^{\dagger}}$, then $\mathrm{Pl}-\# \operatorname{CSP}^{2}(\mathcal{F})$ is tractable. Otherwise, $\mathrm{Pl}$-\#CSP${ }^{2}(\mathcal{F})$ is \#P-hard.

In Figs. 24 and 25, we give Venn diagrams to illustrate the relationship among tractable classes for \#CSP${ }^{2}$ and \#Pl-CSP ${ }^{2}$, respectively.

Proof Outline of Theorem 9.2 The overall plan is to break the proof into two main steps.

The first step is to prove the dichotomy theorem for $\operatorname{Pl}^{-\# C S P}(\mathcal{F})$ when there is at least one nonzero signature of odd arity in $\mathcal{F}$. In this case we can make use of Lemma 10.2 that shows that we can simulate $\operatorname{Pl}-\# \operatorname{CSP}(\mathcal{F})$ by $\operatorname{Pl}-\# \operatorname{CSP}^{2}(\mathcal{F})$ if $\mathcal{F}$ includes a unary signature $[a, b]$ with $a b \neq 0$. Then we can apply the known dichotomy Theorem 9.22 for Pl-\#CSP. However this strategy (provably) cannot work when every signature in $\mathcal{F}$ satisfies parity constraints, as in this case there is no unary signature $[a, b]$ with $a b \neq 0$. For this case we employ other means. This first step of the proof is relatively uncomplicated.

The second step is to deal with the case when all nonzero signatures in $\mathcal{F}$ have even arity. This is where the real difficulties lie. In this case it is impossible to directly construct any unary signature. So we cannot use Lemma 10.2 in this case. But Lemma 10.3 provides a way to simulate $\operatorname{Pl}-\# \operatorname{CSP}(\mathcal{F})$ by $\operatorname{Pl}-\# \operatorname{CSP}^{2}(\mathcal{F})$ in a global fashion, if $\mathcal{F}$ includes some tensor power of the form $[a, b]^{\otimes 2}$ where $a b \neq 0$. Moreover, we have a lucky break (for the complexity of the proof) if $\mathcal{F}$ includes a signature 


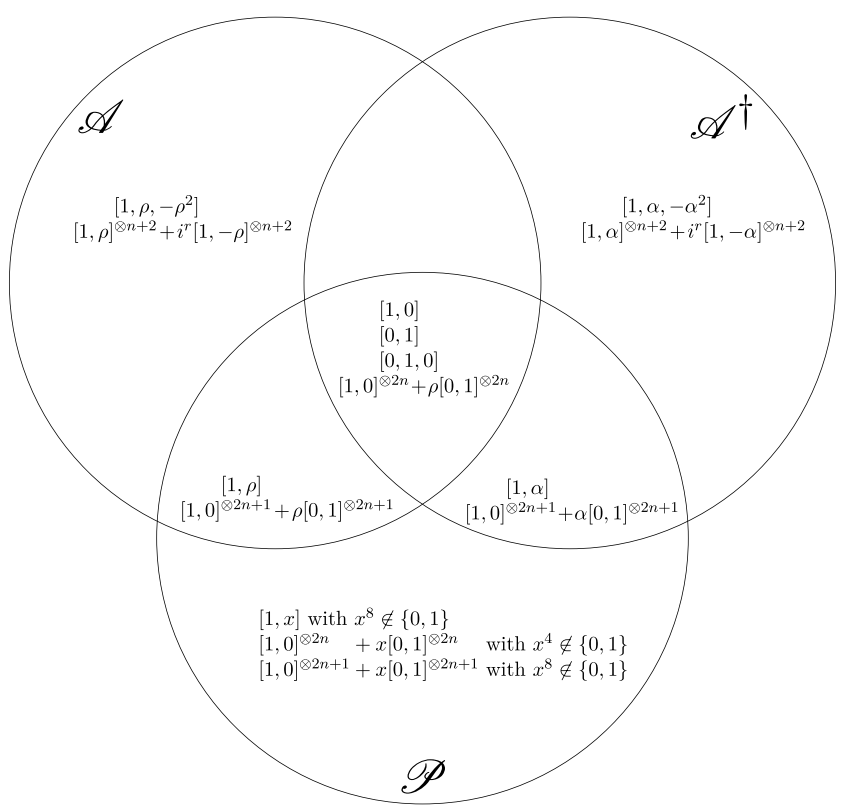

Fig. 24 A Venn diagram of the \#CSP ${ }^{2}$ tractable sets $\mathscr{A}, \mathscr{A}^{\dagger}$, and $\mathscr{P}$. Note that $\rho^{4}=1, \alpha^{4}=-1$, and $n \geq 1$. Excluded are tensor products of unary signatures

that is in $\widehat{\mathscr{M} \backslash}(\mathscr{P} \cup \widetilde{\mathscr{A}})$. In this case, we can construct a special binary signature, and then use Lemma 13.2 to obtain $[1,1]^{\otimes 2}$ by interpolation. This proof uses the theory of cyclotomic fields. This simplifies the proof greatly. For all other cases (when $\mathcal{F}$ has only even arity signatures), the proof gets going in earnest-we will attempt an induction on the arity of signatures.

The lowest arity of this induction will be 2 . We will try to reduce the arity to 2 whenever possible; however for many cases an arity reduction to 2 destroys the \#Phardness at hand. Therefore the true basis of this induction proof of Pl-\#CSP ${ }^{2}$ starts with arity 4. Consequently we will first prove a dichotomy theorem for $\mathrm{Pl}-\# \mathrm{CSP}^{2}(f)$, where $f$ is a signature of arity 4. This proof is presented in Section 12. Several tools will be used. These include the rank criterion for redundant signatures, Theorem 9.21 for arity 2 signatures, and a trick we call the Three Stooges by domain pairing.

However in the next step we do not attempt a general Pl-\#CSP ${ }^{2}$ dichotomy for a single signature of even arity. This would have been natural at this point, but it would have been too difficult. We will need some additional leverage by proving a conditional No-Mixing Lemma for pairs of signatures of even arity. So, seemingly taking a detour, we prove that for two signatures $f$ and $g$ both of even arity, that individually belong to some tractable class, but do not belong to a single tractable class in the conjectured $\mathrm{Pl}-\# \mathrm{CSP}^{2}$ dichotomy (that is yet to be proved), the problem $\mathrm{Pl}$-\#CSP${ }^{2}(f, g)$ is \#P-hard. We prove this No-Mixing Lemma for any pair of signatures $f$ and $g$ both of even arity, not restricted to arity 4. Even though at this point we only have a dichotomy for a single signature of arity 4 , we prove this No-Mixing 


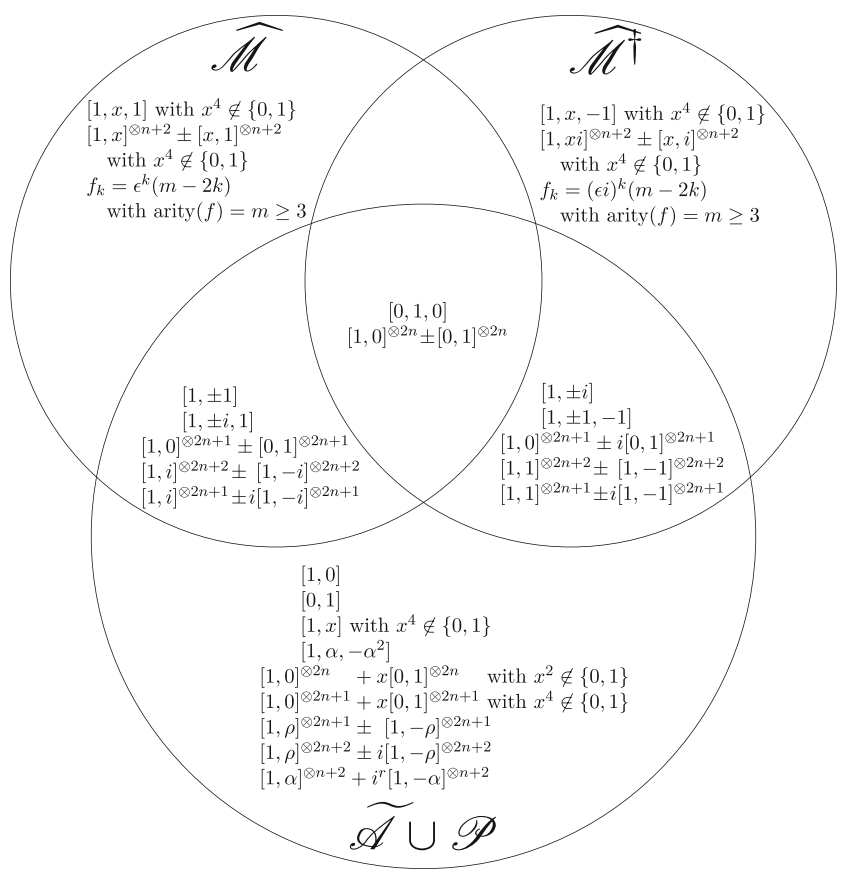

Fig. 25 A Venn diagram of the Pl-\#CSP ${ }^{2}$ tractable sets $\widehat{\mathscr{M}}$ and $\widehat{\mathscr{M}}^{\dagger}$ along with the set $\tilde{\mathscr{A}} \cup \mathscr{P}$ of all tractable \#CSP ${ }^{2}$ signatures. Note that $\rho^{4}=1, \alpha^{4}=-1$, and $n \geq 1$. Excluded are tensor products of unary signatures

Lemma for higher even arity pairs $f$ and $g$ by simulating two signatures $f^{\prime}$ and $g^{\prime}$ of arity 4 that belong to different tractable sets, from that of $\mathrm{Pl}-\# \mathrm{CSP}^{2}(f, g)$. After this arity reduction (within the No-Mixing Lemma), we prove that $\mathrm{Pl} \# \mathrm{CSP}^{2}\left(f^{\prime}, g^{\prime}\right)$ is \#P-hard by the dichotomy for a single signature of arity 4. After this, we prove a No-Mixing Lemma for a set of signatures $\mathcal{F}$ of even arities, which states that if $\mathcal{F}$ is contained in the union of all tractable classes, then it is still \#P-hard unless it is entirely contained in a single tractable class. Note that at this point we still only have a conditional No-Mixing Lemma in the sense that we have to assume every signature in $\mathcal{F}$ belongs to some tractable set.

We then attempt the proof of a Pl-\#CSP ${ }^{2}$ dichotomy for a single signature of arbitrary even arity. This uses all the previous lemmas, in particular the conditional No-Mixing Lemma for a set of signatures. However, after completing the proof of this $\mathrm{Pl}$-\#CSP${ }^{2}$ dichotomy for a single signature of even arity, the conditional No-Mixing Lemma becomes unconditional.

Finally the dichotomy for a single signature of even arity is logically extended to a dichotomy theorem for $\mathrm{Pl}-\# \mathrm{CSP}^{2}(\mathcal{F})$ for a set of signatures where all signatures in $\mathcal{F}$ have even arity. Together with the first main step when $\mathcal{F}$ contains some nonzero signature of odd arity, this completes the proof of Theorem 9.2.

In the rest of this Section 9, we will introduce the operators $\partial$ and $\int$, and give some characterizations of the tractable classes. We will also introduce some 
preliminary lemmas, including one using the domain pairing technique, and list some known dichotomies. In Section 10, we discuss a technique to simulate Pl-\#CSP by Pl-\#CSP ${ }^{2}$. Section 11 proves Theorem 9.2 in the case when $\mathcal{F}$ contains at least one nonzero signature of odd arity. Section 12 proves the base case of the even arity case of Theorem 9.2 when $\mathcal{F}$ consists of a single signature of arity 4 . Section 13 gives an application of cyclotomic field which simplifies the proof of Theorem 9.2 when $\mathcal{F}$ contains a signature in $\widetilde{\mathscr{M}} \backslash(\mathscr{P} \cup \mathscr{\mathscr { A }})$. Section 14 proves the conditional No Mixing lemmas for a pair of signatures of even arity. Section 15 generalizes the No Mixing lemmas to a set of signatures of even arity. Section 16 finishes the proof of Theorem 9.2.

Remark 5 We occasionally make some remarks (such as Remark 6 and Remark 7 in Section 13.2) to explain the complications forced upon the proof by various reasons, and why another more straightforward approach would not succeed. These remarks are not logically necessary to the proof, but hopefully they provide some insight and point out pitfalls in the proof.

In this section we collect a number of simple facts which may appear disparate and unmotivated. They are collected here in one place for the convenience of presenting the proof in later sections. Readers can skim these first and come back to them when needed.

The next lemma is a simple fact that is used many times. It says that the set $\{0,1, i,-1,-i, \infty\}$ is closed set-wise under the mapping $z \mapsto \frac{z+1}{z-1}$ on the extended complex plane $\mathbb{C} \cup\{\infty\}$. The proof is straightforward, so we omit it.

Lemma 9.3 On the extended complex plane $\mathbb{C} \cup\{\infty\}$, the mapping $z \mapsto \frac{z+1}{z-1}$ maps $\{0,1, i,-1,-i, \infty\}$ to itself. In particular, for $x \neq y$ from $\mathbb{C}$, and $\lambda=\frac{x+y}{x-y}$, we have $\lambda^{4} \notin\{0,1\}$ iff $x^{4} \neq y^{4}$ and $x y \neq 0$.

Definition 9.4 (Derivative) Let $f$ and $g$ be two symmetric signatures of arities $n$ and $m$ respectively, and $n>m$. By connecting all $m$ input edges of $g$ to $f$, we get a planar $\{f, g\}$-gate with a signature of arity $n-m$. This derivative signature will be denoted by $\partial_{g}(f)$. If $\mathrm{km}<n$ and we connect $k$ copies of $g$ to $f$, which is the same as forming $\partial_{g}(f)$ sequentially $k$ times, the resulting repeated derivative signature is denoted by $\partial_{g}^{k}(f)$. If $g=[1,0,1]$, we denote $\partial_{g}(f)$ simply by $\partial(f)$.

Calculus: Our proof will make substantial use of an analog calculus using this notion of a derivative. This calculus is essentially a systematic way to calculate the signatures of some gadget constructions. In a Pl-Holant problem Pl-Holant $(\mathcal{G}$ | $\mathcal{F}$ ), if $g \in \mathcal{G}$ and $f \in \mathcal{F}$, then we say that $g$ is from the LHS and $f$ is from the RHS. If $f$ has arity $n$ and $g$ has arity $m$, and $n>m$, then we can form the signature $\partial_{g}(f)$ and $\operatorname{Pl-Holant}\left(\mathcal{G} \mid \mathcal{F} \cup\left\{\partial_{g}(f)\right\}\right) \leq_{T} \operatorname{Pl}$-Holant $(\mathcal{G} \mid \mathcal{F})$. If $m>n$ we can form $\partial_{f}(g)$ and Pl-Holant $\left(\mathcal{G} \cup\left\{\partial_{f}(g)\right\} \mid \mathcal{F}\right) \leq_{T} \operatorname{Pl-Holant}(\mathcal{G} \mid$ $\mathcal{F}$ ). In particular, for $\operatorname{Pl}-\# \operatorname{CSP}^{2}(\mathcal{F}) \equiv \operatorname{Pl}$-Holant $\left(\mathcal{E} \mathcal{Q}_{2} \mid \mathcal{F}\right.$ ) (recall that $\mathcal{E} \mathcal{Q}_{2}=$ $\left\{=2,=_{4},=_{6}, \cdots\right\}$ defined in Section 2.3 in Part I) we have all $\left(=_{2 k}\right)$ from the LHS. In this case if $h \in \mathcal{F}$ with arity $<n$ then we can also form $\partial_{h}(f)$, by 
first moving $h$ to LHS via $(=2) \in \mathcal{E} \mathcal{Q}_{2}$, and then $\operatorname{Pl}_{2} \# \operatorname{CSP}^{2}\left(\mathcal{F} \cup\left\{\partial_{h}(f)\right\}\right) \leq_{T}$ $\mathrm{Pl}-\# \mathrm{CSP}^{2}(\mathcal{F})$. Note that if we discuss $\mathrm{Pl}-\# \operatorname{CSP}^{4}(\mathcal{F}) \equiv \operatorname{Pl}$-Holant $\left(\mathcal{E} \mathcal{Q}_{4} \mid \mathcal{F}\right)$ (recall that $\mathcal{E} \mathcal{Q}_{4}=\left\{=_{4},=8,={ }_{12}, \cdots\right\}$ defined in Section 2.3 in Part I) then this operation $\partial_{h}(f)$ is in general not permissible for $f, h \in \mathcal{F}$, and has to be justified in each individual case, e.g. when $h$ has even arity and one can construct $[1,0,1]^{\otimes 2}$ in the LHS.

To familiarize the readers with this calculus, we list some simple calculations below, which we will use often in our proofs freely without comments. Recall that we use $\epsilon$ to denote \pm 1 .

For any $g$, the operator $\partial_{g}(\cdot)$ is a linear operator. It also depends on $g$ linearly.

By definition $\partial\left(\left[f_{0}, f_{1}, \ldots, f_{n}\right]\right)=\left[f_{0}+f_{2}, f_{1}+f_{3}, \ldots, f_{n-2}+f_{n}\right]$ has arity $n-2$.

1. For $g=\left[g_{0}, g_{1}, \ldots, g_{m}\right]$, we have $\partial_{g}\left(=_{n}\right)=\left[g_{0}, 0, \ldots, 0, g_{m}\right]$ of arity $n-m$, where $n>m$.

2. If $f=[s, t]^{\otimes n}$, then

- $\partial_{[a, b]}^{k}(f)=(a s+b t)^{k}[s, t]^{\otimes n-k}$ if $n>k$.

- $\partial_{[a, b, c]}^{k}(f)=\left(a s^{2}+2 b s t+c t^{2}\right)^{k}[s, t]^{\otimes n-2 k}$ if $n>2 k$;

in particular, $\partial^{k}(f)=\left(s^{2}+t^{2}\right)^{k}[s, t]^{\otimes n-2 k}$.

- $\partial_{=4}^{k}(f)=\left(s^{4}+t^{4}\right)^{k}[s, t]^{\otimes n-4 k}$, if $n>4 k$.

3. Let $f$ be of arity $n$ and $f_{k}=\epsilon^{k}(n-2 k)$ for some $\epsilon= \pm 1$ and for $0 \leq k \leq n$, then

- $\quad \partial(f)$ has arity $n^{\prime}=n-2$ and $(\partial(f))_{k}=2 \epsilon^{k}\left(n^{\prime}-2 k\right)$. If $n$ is odd, then $\partial^{\frac{n-1}{2}}(f)=2^{\frac{n-1}{2}}[1,-\epsilon]$.

- $\partial_{=}(f)$ has arity $n^{\prime \prime}=n-4$ and $\left(\partial_{=}(f)\right)_{k}=2 \epsilon^{k}\left(n^{\prime \prime}-2 k\right)$.

If $n \equiv 1(\bmod 4)$, then $\partial_{=4}^{\frac{n-1}{4}}(f)=2^{\frac{n-1}{4}}[1,-\epsilon]$.

If $n \equiv 3(\bmod 4)$, then $\partial\left(\partial_{=4}^{\frac{n-3}{4}}(f)\right)=2^{\frac{n+1}{4}}[1,-\epsilon]$.

4. Let $f$ be of arity $n$ and $f_{k}=(\epsilon i)^{k}(n-2 k)(0 \leq k \leq n)$, then

- $\partial(f)=4[1, \epsilon i]^{\otimes n-2}$.

- $\partial_{=}(f)$ has arity $m=n-4$ and $\left(\partial_{=}(f)\right)_{k}=2(\epsilon i)^{k}(m-2 k)$.

If $n \equiv 1(\bmod 4)$, then $\partial_{=4}^{\frac{n-1}{4}}(f)=2^{\frac{n-1}{4}}[1,-\epsilon i]$.

If $n \equiv 3(\bmod 4)$, then $\partial\left(\partial_{=4}^{\frac{n-3}{4}}(f)\right)=2^{\frac{n+5}{4}}[1, \epsilon i]$.

Now we define an inverse operator $\int(\cdot)$ to $\partial$. Just like the usual calculus there is a certain non-uniqueness in the expression in an indefinite integral; this non-uniqueness is addressed in Lemma 9.5. One reasonable definition for $\int\left(\left[f_{0}, f_{1}, \ldots, f_{n}\right]\right)$ is $F=$ $\left[F_{0}, F_{1}, \ldots, F_{n+2}\right]$ such that

$$
F_{k}=\sum_{s \geq 0}(-1)^{s} f_{k+2 s}=f_{k}-f_{k+2}+f_{k+4}-\ldots
$$

where we define $f_{k}=0$ for all $k>n$. Clearly $\partial(F)=f$. 
Lemma 9.5 Let $F$ and $G$ be symmetric signatures of arity $n \geq 3$ and suppose $\partial(F)=\partial(G)$. Then $F-G=x[1, i]^{\otimes n}+y[1,-i]^{\otimes n}=[x+y, i(x-y),-(x+$ $\left.y),-i(x-y), \cdots, i^{n}\left(x+(-1)^{n} y\right)\right]$ for some constants $x$ and $y$.

Proof The signature $H=F-G$ satisfies $\partial(H)=0$, and thus satisfies the secondorder recurrence relation $H_{k}+H_{k+2}=0$ for $0 \leq k \leq n-2$. Hence there exist constants $x$ and $y$ such that $H=x[1, i]^{\otimes n}+y[1,-i]^{\otimes n}$.

Note that $x[1, i]^{\otimes n}+y[1,-i]^{\otimes n}$ has the form $[u, v,-u,-v, \ldots]$, where $u=x+y$ and $v=i(x-y)$.

Thus $\int(\cdot)$ is well-defined up to an additive term $x[1, i]^{\otimes n}+y[1,-i]^{\otimes n}$. In this paper, we choose to write the expression $\int(f)$ by the following definition when a certain special expression of $f$ exists. This is more convenient for our proofs.

Definition 9.6 For $n \geq 3, \int(\cdot)$ is a linear operator and

- $\int(0)=0$.

- $\quad$ For $a^{2}+b^{2} \neq 0, \int\left([a, b]^{\otimes n-2}\right)=\frac{1}{a^{2}+b^{2}}[a, b]^{\otimes n}$.

- $\int\left([1, \epsilon i]^{\otimes n-2}\right)$ has arity $n$ and $\left[\int\left([1, \epsilon i]^{\otimes n-2}\right)\right]_{k}=\frac{1}{4}(\epsilon i)^{k}(n-2 k)$.

- If the signature $g$ has arity $n-2$ and $g_{k}=\epsilon^{k}(n-2-2 k)$, then $\int(g)$ has arity $n$ and $\left[\int(g)\right]_{k}=\frac{1}{2} \epsilon^{k}(n-2 k)$.

- If the signature $g$ has arity $n-2$ and $g_{k}=(\epsilon i)^{k}(n-2-2 k)$, then $\int(g)$ has arity $n$ and $\left[\int(g)\right]_{k}=\left(-\frac{n}{2} k+\frac{1}{2} k^{2}\right)(\epsilon i)^{k}$.

Clearly for all $f$ where $\int(f)$ is given in the above definition, $\partial\left[\int(f)\right]=f$.

When we prove the dichotomy theorem for $\mathrm{Pl}^{-\mathrm{CSP}^{2}}(f)$, where $f$ has arity $n$, we can get a signature $f^{\prime}$ of arity $n-2$ by taking a self loop with $f$, i.e., $f^{\prime}=\partial(f)$. Clearly $\mathrm{Pl}-\# \operatorname{CSP}^{2}\left(f^{\prime}\right) \leq_{T} \mathrm{Pl}-\# \operatorname{CSP}^{2}(f)$. If $f^{\prime} \notin \mathscr{P} \cup \mathscr{\mathscr { A }} \cup \widetilde{\mathscr{M}}$, then by induction $\mathrm{Pl}$ \#CSP${ }^{2}\left(f^{\prime}\right)$ is \#P-hard. Thus Pl-\#CSP ${ }^{2}(f)$ is also \#P-hard. Definition 9.6 allows us to write down an explicit expression for $\int\left(f^{\prime}\right)$ for all cases when $f^{\prime} \in \mathscr{P} \cup \widetilde{\mathscr{A}} \cup \widetilde{\mathscr{M}}$.

The following is an explicit list of $\int\left(f^{\prime}\right)$ for $f^{\prime}=\partial(f) \in \mathscr{P} \cup \widetilde{\mathscr{A}} \cup \widetilde{\mathscr{M}}$. We can recover $f$ up to the constants $x, y$ from $\partial(f)$ by Lemma 9.5. This list is for the convenience of the readers.

Proposition 9.7 (Explicit List for $\int\left(f^{\prime}\right)$ )

- $\int\left(f^{\prime}\right) \equiv 0$ if $f^{\prime} \equiv 0$.

- $\int\left([1,0]^{\otimes n-2}+a[0,1]^{\otimes n-2}\right)=[1,0]^{\otimes n}+a[0,1]^{\otimes n}$.

- $\int\left([1, \gamma]^{\otimes n-2}+i^{r}[1,-\gamma]^{\otimes n-2}\right)=\frac{1}{1+\gamma^{2}}[1, \gamma]^{\otimes n}+\frac{i^{r}}{1+\gamma^{2}}[1,-\gamma]^{\otimes n}$ where $\gamma^{2} \neq$ $-1, \gamma^{8}=1$.

- $\int\left([s, t \rho]^{\otimes n-2} \pm[t, s \rho]^{\otimes n-2}\right)=\frac{1}{s^{2}+\rho^{2} t^{2}}[s, \rho t]^{\otimes n} \pm \frac{1}{\rho^{2} s^{2}+t^{2}}[t, \rho s]^{\otimes n}$, where $\rho^{4}=$ $1, s t \neq 0, s^{4} \neq t^{4}$.

- $\left[\int\left(f^{\prime}\right)\right]_{k}=\frac{1}{2} \epsilon^{k}(n-2 k)$ if $f^{\prime}$ has arity $n-2$ and $f_{k}^{\prime}=\epsilon^{k}(n-2-2 k)$.

- $\left[\int\left(f^{\prime}\right)\right]_{k}=\frac{1}{4}(\epsilon i)^{k}(n-2 k)$ if $f^{\prime}$ has arity $n-2$ and $f^{\prime}=[1, \epsilon i]^{\otimes n-2}$. 
- $\left[\int\left(f^{\prime}\right)\right]_{k}=\frac{1}{4}\left[i^{k}+i^{r}(-i)^{k}\right](n-2 k)$ if $f^{\prime}$ has arity $n-2$ and $f^{\prime}=[1, i]^{\otimes n-2}+$ $i^{r}[1,-i]^{\otimes n-2}$.

- $\left[\int\left(f^{\prime}\right)\right]_{k}=\left(-\frac{n}{2} k+\frac{1}{2} k^{2}\right)(\epsilon i)^{k}$ if $f^{\prime}$ has arity $n-2$ and $f_{k}^{\prime}=(\epsilon i)^{k}(n-2-2 k)$.

Proof Note that all the $f^{\prime}$ in the list are linear combinations of the signatures in Definition 9.6 and $\int(\cdot)$ is a linear operator. So the list follows from Definition 9.6 directly.

The following lemma is used to determine whether a binary signature belongs to various tractable sets $\mathscr{P}, \mathscr{A}, \mathscr{A}^{\dagger}, \widehat{\mathscr{M}}$, and $\widehat{\mathscr{M}}^{\dagger}$. It can be proved directly by the definition.

Lemma 9.8 For any binary symmetric signature $f$,

- $f \in \mathscr{P}$ iff $f=[a, 0, c]$ or $f=[0, b, 0]$ or $f=[a, b]^{\otimes 2}$.

- $f \in \mathscr{A}$ iff up to a scalar, $f=\left[1, \rho,-\rho^{2}\right]$ where $\rho^{4}=1$, or $[0,1,0]$, or $[1,0, \rho]$ where $\rho^{4}=1$, or $[x, y]^{\otimes 2}$ where $\left(x^{4}=y^{4} \neq 0\right.$ or $\left.x y=0\right)$.

- $f \in \mathscr{A}^{\dagger}$ iff up to a scalar, $f=\left[1, \alpha,-\alpha^{2}\right]$ where $\alpha^{4}=-1$, or $[0,1,0]$, or $[1,0, \rho]$ where $\rho^{4}=1$, or $[x, y]^{\otimes 2}$ where $\left(x^{4}=-y^{4} \neq 0\right.$ or $\left.x y=0\right)$.

- $f \in \widehat{\mathscr{M}}$ iff $f=[a, b, a]$ or $[a, 0,-a]$.

- $f \in \widehat{\mathscr{M}}^{\dagger}$ iff $f=[a, b,-a]$ or $[a, 0, a]$.

Proof The condition for $f \in \mathscr{P}$ follows from Definition 2.6.

The condition for $f \in \mathscr{A}$ follows from the lists after Definition 2.5.

If $f \in \mathscr{A}^{\dagger}$, then $\left[\begin{array}{ll}1 & 0 \\ 0 & \alpha\end{array}\right]^{\otimes 2} f \in \mathscr{A}$. Then the condition for $f \in \mathscr{A}^{\dagger}$ follows from the condition $f \in \mathscr{A}$.

By Proposition 2.8, one binary signature is in $\mathscr{M}$ iff it has the form $[x, 0, y]$ or $[0, x, 0]$. After the holographic transformation using $\left[\begin{array}{ll}1 & 1 \\ 1 & -1\end{array}\right]$, we get the condition for $f \in \widehat{\mathscr{M}}$.

Then after the holographic transformation using $\left[\begin{array}{ll}1 & 0 \\ 0 & i\end{array}\right]$, we get the condition for $f \in \widehat{\mathscr{M}^{\dagger}}$.

Corollary 9.9 gives some necessary conditions for a binary signature to belong to a tractable set.

Corollary 9.9 For any binary signature $f=[a, b, c]$,

- $f \in \mathscr{P} \Longrightarrow f$ satisfies either the parity constraints or $b^{2}=a c$.

- $f \in \mathscr{A} \Longrightarrow a^{2}=c^{2}$ or $b=0$. If $f \in \mathscr{A} \backslash \mathscr{P}$, then $f=\left[1, \rho,-\rho^{2}\right], \rho^{4}=1$.

- $f \in \mathscr{A}^{\dagger} \Longrightarrow a^{2}=-c^{2}$ or $b=0$. If $f \in \mathscr{A}^{\dagger} \backslash \mathscr{P}$, then $f=\left[1, \alpha,-\alpha^{2}\right]$, $\alpha^{4}=-1$.

- $f \in \tilde{\mathscr{A}} \Longrightarrow$ the norms of all nonzero entries are equal.

- $f \in \widetilde{\mathscr{M}} \Longrightarrow a^{2}=c^{2}$. 
Furthermore, all signatures in each tractable set satisfy a second-order recurrence relation.

Definition 9.10 Let $f=\left[f_{0}, f_{1}, \ldots, f_{n}\right]$. If there exist constants $a, b$ and $c$, not all zero, such that $a f_{k}-b f_{k+1}+c f_{k+2}=0$ for $0 \leq k \leq n-2$, then we say $f$ has type $\langle a, b, c\rangle$, and it is denoted by $f \in\langle a, b, c\rangle$.

For a non-degenerate symmetric signature $f$ of arity at least 3 , if $f$ has type $\langle a, b, c\rangle$, its type is uniquely determined up to a nonzero multiple. The next lemma states this type information for the various tractable sets. We can use the lemma to check whether a symmetric signature can possibly be in a tractable set.

Lemma 9.11 Let $f \in \mathscr{P} \cup \widetilde{\mathscr{A}} \cup \widetilde{\mathscr{M}}$ be non-degenerate and have arity $\geq 3$.

- If $f \in \mathscr{P}$ then $f \in\langle 0,1,0\rangle$.

- If $f \in \mathscr{A}$ then $f \in\langle 0,1,0\rangle$ or $f \in\langle 1,0, \pm 1\rangle$. If $f \in \mathscr{A} \backslash \mathscr{P}$ then $f \in$ $\langle 1,0, \pm 1\rangle$.

- If $f \in \mathscr{A}^{\dagger}$ then $f \in\langle 0,1,0\rangle$ or $f \in\langle 1,0, \pm i\rangle$. If $f \in \mathscr{A}^{\dagger} \backslash \mathscr{P}$ then $f \in$ $\langle 1,0, \pm i\rangle$.

- If $f \in \widehat{\mathscr{M}}$ then $f \in\langle 0,1,0\rangle$ or $f \in\langle 1, c, 1\rangle$. If $f \in \widehat{\mathscr{M}} \backslash(\mathscr{P} \cup \widetilde{\mathscr{A}})$ then $f \in\langle 1, c, 1\rangle$ with $c \neq 0$.

- If $f \in \widehat{\mathscr{M}^{\dagger}}$ then $f \in\langle 0,1,0\rangle$ or $f \in\langle 1, c,-1\rangle$. If $f \in \widehat{\mathscr{M}^{\dagger}} \backslash(\mathscr{P} \cup \tilde{\mathscr{A}})$ then $f \in\langle 1, c,-1\rangle$ with $c \neq 0$.

Proof By Definition 2.6, if $f \in \mathscr{P}$ is non-degenerate and has arity $\geq 3$, then $f$ has the form $[a, 0, \cdots, 0, b]$. Thus $f \in\langle 0,1,0\rangle$.

By the lists after Definition 2.5, $f \in \mathscr{A}$ then $f \in\langle 0,1,0\rangle$ or $f \in\langle 1,0, \pm 1\rangle$. Moreover, if $f \in \mathscr{A} \backslash \mathscr{P}$ then $f \in\langle 1,0, \pm 1\rangle$.

After the holographic transformation using $\left[\begin{array}{ll}1 & 0 \\ 0 & \alpha\end{array}\right]$, we get the conditions for $f \in$ $\mathscr{A}^{\dagger}$ and $f \in \mathscr{A}^{\dagger} \backslash \mathscr{P}$.

By Proposition 2.8, $f^{\prime} \in \mathscr{M}$ iff $f^{\prime} \in\langle x, 0, y\rangle$ or $f^{\prime} \in\langle 0,1,0\rangle$. Then after the holographic transformation using $\left[\begin{array}{ll}1 & 1 \\ 1 & -1\end{array}\right], f \in \widehat{\mathscr{M}}$ iff $f \in\langle x+y, x-y, x+y\rangle$. If $x+y \neq 0$, then let $c=\frac{x-y}{x+y}$ and $f \in\langle 1, c, 1\rangle$ after the scalar $x+y$. Otherwise $f \in\langle 0,1,0\rangle$ after the scalar $x-y$. Note that if $f \in\langle 0,1,0\rangle$, then $f \in \mathscr{P}$. Thus if $f \in \widehat{\mathscr{M}} \backslash(\mathscr{P} \cup \widetilde{\mathscr{A}})$ then $f \in\langle 1, c, 1\rangle$ with $c \neq 0$.

After the holographic transformation using $\left[\begin{array}{ll}1 & 0 \\ 0 & i\end{array}\right]$, we get the conditions for $f \in$ $\widehat{\mathscr{M}^{\dagger}}$ and $f \in \widehat{\mathscr{M}^{\dagger}} \backslash(\mathscr{P} \cup \widetilde{\mathscr{A}})$.

The following two corollaries follow from Lemma 9.8 for the binary case, and Lemma 9.11 for arity $n \geq 3$. 
Corollary 9.12 If $f \in \mathscr{A} \backslash \mathscr{P}$, then $f \notin \mathscr{A}^{\dagger}$. Similarly, If $f \in \mathscr{A}^{\dagger} \backslash \mathscr{P}$, then $f \notin \mathscr{A}$.

Corollary 9.13 If $f \in \widehat{\mathscr{M} \backslash}(\mathscr{P} \cup \widetilde{\mathscr{A}})$, then $f \notin \widehat{\mathscr{M}^{\dagger}}$. Similarly, if $f \in \widehat{\mathscr{M}}^{\dagger} \backslash(\mathscr{P} \cup \tilde{\mathscr{A}})$, then $f \notin \widehat{\mathscr{M}}$.

The following lemma gives a characterization of $\widetilde{\mathscr{M}} \backslash(\mathscr{P} \cup \widetilde{\mathscr{A}})$.

Lemma 9.14 Let $f=\left[f_{0}, \ldots, f_{n}\right]$ be a symmetric signature of arity $n$. Then $f \in$ $\widehat{\mathscr{M} \backslash} \backslash(\mathscr{P} \cup \mathscr{A})$ iff

- $n=2$ and $f=\lambda[1, a, 1]$, where $a^{4} \notin\{0,1\}$ and $\lambda \neq 0$; or

- $n \geq 3$ and $f=[s, t]^{\otimes n} \pm[t, s]^{\otimes n}$, where $s t \neq 0$ and $s^{4} \neq t^{4}$; or

- $n \geq 3$ and $f_{k}=\lambda \epsilon^{k}(n-2 k)$ for $0 \leq k \leq n$, where $\lambda \neq 0$.

Similarly, $f \in \widehat{\mathscr{M}^{\dagger}} \backslash(\mathscr{P} \cup \widetilde{\mathscr{A}})$ iff

- $n=2$ and $f=\lambda[1, b,-1]$, where $b^{4} \notin\{0,1\}$ and $\lambda \neq 0$; or

- $n \geq 3$ and $f=[s, t i]^{\otimes n} \pm[t, \text { si }]^{\otimes n}$ where $s t \neq 0$ and $s^{4} \neq t^{4}$; or

- $n \geq 3$ and $f_{k}=\lambda(\epsilon i)^{k}(n-2 k)$ for $0 \leq k \leq n$, where $\lambda \neq 0$.

Proof We prove the lemma for $\widehat{\mathscr{M}}$. The proof for $\widehat{\mathscr{M}}^{\dagger}$ follows from a holographic transformation by $\left[\begin{array}{ll}1 & 0 \\ 0 & i\end{array}\right]$.

By Lemma 9.8, a binary symmetric signature $f \in \widehat{\mathscr{M}}$ has the form $[a, b, a]$ or $[a, 0,-a]$. Since $[a, 0,-a] \in \mathscr{A}$ is a multiple of $[1,0,-1]$, we exclude it. For $[a, b, a]$, if $a b=0$, then $f \in \mathscr{P}$. Also if $a^{4}=b^{4}$, then $[a, b, a] \in \mathscr{A}$, being a multiple of $[1, \pm 1]^{\otimes 2}$ or $[1, \pm i, 1]$. This gives the form $f=\lambda[1, b, 1]$ with $b^{4} \notin\{0,1\}$ and $\lambda \neq 0$. Conversely, any $f$ of this form belongs to $\widehat{\mathscr{M} \backslash}(\mathscr{P} \cup \widetilde{\mathscr{A}})$.

For arity $n \geq 3$, by Proposition 2.8 and the holographic transformation using $\left[\begin{array}{ll}1 & 1 \\ 1 & -1\end{array}\right], f \in \widehat{\mathscr{M}}$ iff $f$ takes the form $[s, t]^{\otimes n} \pm[t, s]^{\otimes n}$ or $f_{k}=\lambda \epsilon^{k}(n-2 k)$. For the latter case $f \in \widehat{\mathscr{M}} \backslash(\mathscr{P} \cup \widetilde{\mathscr{A}})$ follows from its type $\langle 1, \pm 2,1\rangle$.

For $f=[s, t]^{\otimes n} \pm[t, s]^{\otimes n}$, if $s t=0$, then $f \in \mathscr{P}$. If $s^{2}=t^{2}$, then $f$ is degenerate, thus $f \in \mathscr{P}$. If $s^{2}=-t^{2}$, then $f \in \mathscr{A}$. Conversely, if $s t \neq 0$ and $s^{4} \neq t^{4}$, then $f$ is non-degenerate and $f_{k}$ has type $\left\langle 1, \frac{s}{t}+\frac{t}{s}, 1\right\rangle$. Note that $\frac{s}{t}+\frac{t}{s} \neq 0$ by $s^{4} \neq t^{4}$. Thus $f \in \widehat{\mathscr{M} \backslash} \backslash(\mathscr{P} \cup \widetilde{\mathscr{A}})$ by Lemma 9.11 .

By the second-order recurrence relation of the signatures in $\widetilde{\mathscr{M}} \backslash(\mathscr{P} \cup \widetilde{\mathscr{A}})$, we have the following lemma that will be used in the proof of Theorem 11.11. Recall that $\widetilde{\mathscr{M}}=\widehat{\mathscr{M}} \cup \widehat{\mathscr{M}^{\dagger}}$.

Corollary 9.15 If $f \in \widetilde{\mathscr{M}} \backslash(\mathscr{P} \cup \widetilde{\mathscr{A}})$, then $f$ does not satisfy the parity constraints.

Proof For $f \in \widehat{\mathscr{M} \backslash} \backslash(\mathscr{P} \cup \tilde{\mathscr{A}})$, if $f$ has arity 2 , then $f=\lambda[1, a, 1]$ for some $\lambda \neq 0$, $a^{4} \neq 0,1$ by Lemma 9.14 . Thus it does not satisfy the parity constraints.

For $f$ with arity $n \geq 3$, by Lemma 9.11 , there exists a constants $c \neq 0$ such that $f \in\langle 1, c, 1\rangle$. Note that there exists $f_{k} \neq 0$, where $1 \leq k \leq n-1$ by $f \notin \mathscr{P}$. If $f$ 
satisfies the parity constraints, then $f_{k-1}=f_{k+1}=0$. Moreover, by $f_{k-1}-c f_{k}+$ $f_{k+1}=0$, we have $c=0$. This is a contradiction.

The proof for $f \in \widehat{\mathscr{M}^{\dagger}} \backslash(\mathscr{P} \cup \widetilde{\mathscr{A}})$ follows from a holographic transformation by $\left[\begin{array}{ll}1 & 0 \\ 0 & i\end{array}\right]$.

The following lemma gives a characterization of nonzero signatures in $\widehat{\mathscr{M}}$. A GEN-EQ is a signature of the form $f=[a, 0, \ldots, 0, b]$, called a generalized equality (with $a=0$ or $b=0$ allowed.)

Lemma 9.16 A GEN-EQ signature $f$ is in $\widehat{\mathscr{M}}$ iff $f=\lambda[1,0, \ldots, 0, \pm 1]$, for some $\lambda$. Suppose $f$ is a symmetric signature that is not a GEN-EQ. Then $f \in \widehat{\mathscr{M}}$ iff $f$ satisfies a second-order recurrence $f_{k}-c f_{k+1}+f_{k+2}=0($ for $0 \leq k \leq \operatorname{arity}(f)-2)$ and the following conditions hold.

If $f$ has arity $2 n$, then

- $f_{n-k}=f_{n+k}($ for $0 \leq k \leq n), f_{n} \neq 0, c=\frac{2 f_{n-1}}{f_{n}} ;$ or

- $f_{n-k}=-f_{n+k}($ for $0 \leq k \leq n), f_{n-1} \neq 0, c=\frac{f_{n-2}}{f_{n-1}}$.

If $f$ has arity $2 n+1$, then

- $f_{n-k}=f_{n+1+k}($ for $0 \leq k \leq n), f_{n} \neq 0, c=\frac{f_{n-1}}{f_{n}}+1$; or

- $f_{n-k}=-f_{n+1+k}($ for $0 \leq k \leq n), f_{n} \neq 0, c=\frac{f_{n-1}}{f_{n}}-1$.

Proof By Proposition 2.8 and the holographic transformation using $\left[\begin{array}{ll}1 & 1 \\ 1 & -1\end{array}\right]$, symmetric signatures in $\widehat{\mathscr{M}}$ have the following forms, $f=[s, t]^{\otimes m} \pm[t, s]^{\otimes m}$, or $f_{k}=\lambda \epsilon^{k}(m-2 k)(0 \leq k \leq m)$. A GEN-EQ $f \in \widehat{\mathscr{M}}$ iff it takes the first form with $s t=0$. Suppose $f$ is not a GEN-EQ, then we have $s t \neq 0$ in the first form. In particular $f$ is not identically zero. In both forms, $f$ satisfies a second-order recurrence $f_{k}-c f_{k+1}+f_{k+2}=0(0 \leq k \leq m-2)$, for some $c$. For example in the first form with a tensor sum, the product of the eigenvalues $s / t \cdot t / s=1$.

We prove the lemma for the case that $m=2 n$ is even (the odd-arity case is similar and we omit it here).

If $f=[s, t]^{\otimes 2 n}+[t, s]^{\otimes 2 n}$, we have the symmetry $f_{n+k}=f_{n-k}$. Thus $f_{n-1}=$ $f_{n+1}$ and $c f_{n}=2 f_{n-1}$. If $f_{n}=0$, then $f$ is identically zero, a contradiction. Therefore, we have $c=\frac{2 f_{n-1}}{f_{n}}$.

For $f=[s, t]^{\otimes 2 n}-[t, s]^{\otimes 2 n}$, or $f_{k}=\lambda \epsilon^{k}(2 n-2 k)$, we have $f_{n+k}=-f_{n-k}$. Thus we have $f_{n}=0$ and $c f_{n-1}=f_{n-2}$. If $f_{n-1}=0$, then $f$ is identically zero, a contradiction. Therefore, we have $c=\frac{f_{n-2}}{f_{n-1}}$.

Conversely, the second-order recurrence $f_{k}-c f_{k+1}+f_{k+2}=0$ gives the expression $f=c_{1}[s, t]^{\otimes 2 n}+c_{2}[t, s]^{\otimes 2 n}$, or in the double root case when $c=\epsilon 2$, we have the form $f_{k}=\lambda \epsilon^{k}(2 n-\mu k)$. If $f_{n+k}=-f_{n-k}$, then $f_{n}=0$, the double root case must be $f_{k}=\lambda \epsilon^{k}(2 n-2 k)$, and the tensor sum takes the form $f=[s, t]^{\otimes 2 n}-[t, s]^{\otimes 2 n}$. If $f_{n+k}=f_{n-k}$, then we only have the form $f=$ $[s, t]^{\otimes 2 n}+[t, s]^{\otimes 2 n}$. 
For odd arity, the proof is similar. We omit it here.

Corollary 9.17 If $f \in \widehat{\mathscr{M}}^{\dagger}$ has even arity $2 n$, then

$f_{0}=f_{2 n}, f_{1}=-f_{2 n-1}, f_{2}=f_{2 n-2}, \ldots ;$ or $f_{0}=-f_{2 n}, f_{1}=f_{2 n-1}, f_{2}=-f_{2 n-2}, \ldots$

In other words, for all $0 \leq k \leq 2 n$,

$$
f_{k}=f_{2 n-k} \quad \text { or } \quad f_{k}=-f_{2 n-k}
$$

and the signs strictly alternate according to $k$ in either cases.

Proof By definition, $\widehat{\mathscr{M}^{\dagger}}=\left[\begin{array}{ll}1 & 0 \\ 0 & i\end{array}\right]^{\otimes 2 n} \widehat{\mathscr{M}}$. By Lemma 9.16, we have $i^{n-k} f_{n-k}=$ $\epsilon i^{n+k} f_{n+k}$ for all $k$. The Corollary follows.

The discussion above is a characterization of symmetric tractable signatures. In more detail, we characterize the binary symmetric tractable signatures in Lemma 9.8 and Corollary 9.9. For symmetric signatures of arity $\geq 3$, we use the type of the second-order recurrence relation to characterize them in Lemma 9.11. More characterizations are given in Lemma 9.14 and 9.16. Moreover, by Definition 2.6, all unary signatures are in $\mathscr{P}$. If a unary signature satisfies the parity constraints, then it is in $\mathscr{P} \cap \mathscr{A} \cap \mathscr{A}^{\dagger}$, but is not in $\widehat{\mathscr{M}} \cup \widehat{\mathscr{M}}^{\dagger}$. We summarize these relationships among the tractable signatures in Figs. 24 and 25.

In the proof of $\mathrm{Pl}$-\#CSP${ }^{2}$ dichotomy, we often use the following Corollary. It gives a characterization of a signature of arity 4 in $\widetilde{\mathscr{M}}$. It follows directly from Lemma 9.16 and the definition of $\mathscr{\mathscr { M }}^{\dagger}$.

Corollary 9.18 An arity 4 signature $f \in \widehat{\mathscr{M}}$ has one of the following forms:

- $\quad[u, v, w, v, u]$ and $(u+w) w=2 v^{2}$;or

- $\quad[u, v, 0,-v,-u]$.

An arity 4 signature $f \in \widehat{\mathscr{M}^{\dagger}}$ has one of the following forms:

- $\quad[u, v, w,-v, u]$ and $(u-w) w=2 v^{2}$,

- $\quad[u, v, 0, v,-u]$.

The following lemma can be proved by domain pairing. We can use it to derive \#P-hardness of Pl-\#CSP ${ }^{2}$ problems by applying the known dichotomy of Pl-\#CSP.

Lemma 9.19 Suppose $f=\left[f_{0}, f_{1}, \ldots, f_{2 n}\right]$ is a symmetric signature of arity $2 n$. Let $g=\left[f_{0}, f_{2}, \ldots, f_{2 n}\right]$ be a symmetric signature of arity $n$ consisting of all even indexed entries of $f$. Then

$$
\mathrm{Pl} \text { \#CSP }(g) \leq \mathrm{Pl}-\# \mathrm{CSP}^{2}(f) .
$$


Proof For any instance of Pl-\#CSP $(g)$, we replace each edge $e$ by two edges that connect the same incident nodes of $e$. For each variable node that is connected to $k$ edges, we replace its label $=k$ by $=2 k$. We replace each occurrence of $g$ by $f$ as a constraint. Then the new instance is a problem in $\mathrm{Pl}_{-1} \mathrm{CSP}^{2}(f)$ and has the same value as the given instance of $\operatorname{Pl} \# \operatorname{CSP}(g)$, because $g_{k}=f_{2 k}$. Note that the values $f_{2 k+1}$ with an odd index contribute nothing to the partition function in this instance.

The case when $f=[1, i]^{\otimes 4}+a[1,-i]^{\otimes 4}$ poses some special difficulty, mainly because $\partial(f)$ is identically 0 . The following lemma shows that in this case, with $a \neq 0$, we can construct $[1,0,-1]^{\otimes 2}$ in the LHS in a Pl-Holant problem with $f$ on the RHS. Its utility is that after a holographic transformation by $\left[\begin{array}{ll}1 & 0 \\ 0 & i\end{array}\right]$ or by $\left[\begin{array}{ll}1 & 1 \\ i & -i\end{array}\right]=\left[\begin{array}{ll}1 & 0 \\ 0 & i\end{array}\right]\left[\begin{array}{ll}1 & 1 \\ 1 & -1\end{array}\right]$ we have $[1,0,1]^{\otimes 2}$ on the LHS.

Lemma 9.20 Let $\mathcal{F}$ be a set of signatures containing $f=[1, i]^{\otimes 4}+a[1,-i]^{\otimes 4}$. Then

$$
\operatorname{Pl-Holant}\left([1,0,-1]^{\otimes 2} \cup \mathcal{E} \mathcal{Q}_{2} \mid \mathcal{F}\right) \equiv \operatorname{Pl} \# \operatorname{CSP}^{2}(\mathcal{F})
$$

Proof Suppose $a \neq-1$ and consider the gadget in Fig. 26a. We assign $f$ to the circle vertex and $=4$ to the square vertices. This gives $(1+a)[1,0,-1]^{\otimes 2}$ on the left as desired.

Otherwise $a=-1$. Consider the gadget in Fig. 26b. We assign $f$ to the circle vertices and $={ }_{4}$ to the square vertices. This gives $-8[1,0,-1]^{\otimes 2}$ on the left as desired.

Next we state a couple of complexity dichotomy theorems that were previously shown $[13,31]$. They are also quoted as Theorem 2.24 in Section 2 of Part I. Here we restate them for easier reference. The first is a dichotomy theorem about Boolean domain spin systems (counting complex weighted graph homomorphisms) on degree prescribed graphs. It includes $\mathrm{Pl}-\# \mathrm{CSP}^{2}(f)$, where $f$ is a symmetric binary signature, as a special case.

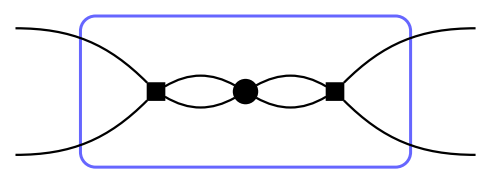

(a)

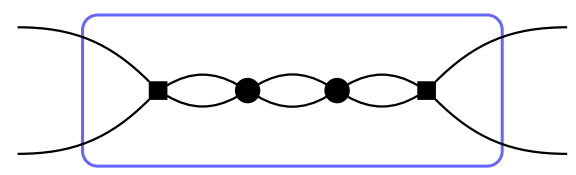

(b)

Fig. 26 Two gadgets used to obtain $[1,0,-1]^{\otimes 2}$. The circle vertices are assigned $f$ and the square vertices are assigned $=4$ 
Theorem 9.21 Let $S \subseteq \mathbb{Z}^{+}$contain $k \geq 3$, let $\mathcal{G}=\left\{=_{k} \mid k \in S\right\}$, and let $d=\operatorname{gcd}(S)$. Further suppose that $f_{0}, f_{1}, f_{2} \in \mathbb{C}$. Then Pl-Holant $\left(\left[f_{0}, f_{1}, f_{2}\right] \mid \mathcal{G}\right)$ is \#P-hard unless one of the following conditions holds:

1. $f_{0} f_{2}=f_{1}^{2}$;

2. $f_{0}=f_{2}=0$;

3. $f_{1}=0$;

4. $f_{0} f_{2}=-f_{1}^{2}$ and $f_{0}^{d}=-f_{2}^{d} \neq 0$;

5. $f_{0}^{d}=f_{2}^{d} \neq 0$.

In any exceptional case, the problem is computable in polynomial time.

Theorem 9.21 is explicit and easy to apply. Conceptually, it can be restated as Theorem $9.21^{\prime}$, which supports the putative form of a Pl-\#CSP ${ }^{d}$ dichotomy (which is false, by Theorem 8.1 of Part I, for $d>2$ ).

Theorem 9.21' Let $S \subseteq \mathbb{Z}^{+}$contain $k \geq 3$, let $\mathcal{G}=\left\{={ }_{k} \mid k \in S\right\}$, and let $d=\operatorname{gcd}(S)$. Further suppose that $f$ is a non-degenerate, symmetric, complex-valued binary signature in Boolean variables. Then Pl-Holant $(f \mid \mathcal{G})$ is \#P-hard unless $f$ satisfies one of the following conditions, in which case, the problem is computable in polynomial time:

1. there exists $T \in \mathcal{T}_{4 d}$ such that $T^{\otimes 2} f \in \mathscr{A}$;

2. $f \in \mathscr{P}$;

3. there exists $T \in \mathcal{T}_{2 d}$ such that $T^{\otimes 2} f \in \widehat{\mathscr{M}}$.

The following theorem is the dichotomy theorem of $\operatorname{Pl} \# \operatorname{CSP}(\mathcal{F})$, where $\mathcal{F}$ is a set of symmetric signatures. This is also quoted as Theorem 2.25 in Part I.

Theorem 9.22 (Theorem 9.3 in [26]) Let $\mathcal{F}$ be any set of symmetric, complex-valued signatures in Boolean variables. Then $\mathrm{Pl}-\mathrm{ASP}(\mathcal{F})$ is $\#$ P-hard unless $\mathcal{F} \subseteq \mathscr{A}, \mathcal{F} \subseteq$ $\mathscr{P}$, or $\mathcal{F} \subseteq \widehat{\mathscr{M}}$, in which case the problem is computable in polynomial time.

We repeat the definition of redundant matrices in Section 2.7.

Definition 9.23 (Definition 32, p. 1692 in [11]) A 4-by-4 matrix is redundant if its middle two rows and middle two columns are the same.

An example of a redundant matrix is the signature matrix of a symmetric arity 4 signature.

Definition 9.24 (Definition 33, p. 1693 in [11]) The signature matrix of a symmetric arity 4 signature $f=\left[f_{0}, f_{1}, f_{2}, f_{3}, f_{4}\right]$ is

$$
M_{f}=\left[\begin{array}{llll}
f_{0} & f_{1} & f_{1} & f_{2} \\
f_{1} & f_{2} & f_{2} & f_{3} \\
f_{1} & f_{2} & f_{2} & f_{3} \\
f_{2} & f_{3} & f_{3} & f_{4}
\end{array}\right] .
$$


This definition extends to an asymmetric signature $g$ as

$$
M_{g}=\left[\begin{array}{llll}
g^{0000} & g^{0010} & g^{0001} & g^{0011} \\
g^{0100} & g^{0110} & g^{0101} & g^{0111} \\
g^{1000} & g^{1010} & g^{1001} & g^{1011} \\
g^{1100} & g^{1110} & g^{1101} & g^{1111}
\end{array}\right] \text {. }
$$

When we present $g$ as an $\mathcal{F}$-gate, we order the four external edges ABCD counterclockwise. In $M_{g}$, the row index bits are ordered $\mathrm{AB}$ and the column index bits are ordered DC, in reverse order. This is for convenience so that the signature matrix of the linking of two arity $4 \mathcal{F}$-gates is the matrix product of the signature matrices of the two $\mathcal{F}$-gates.

If $M_{g}$ is redundant, we also define the compressed signature matrix of $g$ as

$$
\widetilde{M}_{g}=\left[\begin{array}{llll}
1 & 0 & 0 & 0 \\
0 & \frac{1}{2} & \frac{1}{2} & 0 \\
0 & 0 & 0 & 1
\end{array}\right] M_{g}\left[\begin{array}{lll}
1 & 0 & 0 \\
0 & \frac{1}{2} & 0 \\
0 & \frac{1}{2} & 0 \\
0 & 0 & 1
\end{array}\right] .
$$

The definition of compressed signature matrix is a slight change from [26] where $\widetilde{M}_{g}\left[\begin{array}{lll}1 & 0 & 0 \\ 0 & 2 & 0 \\ 0 & 0 & 1\end{array}\right]$ is called by that name. It does not affect the following lemma. We repeat the following lemma from [26], which is very convenient to apply.

Lemma 9.25 (Corollary 3.8 in [26]) Let $f$ be an arity 4 signature with complex weights. If $M_{f}$ is redundant and $\widetilde{M_{f}}$ is nonsingular, then $\mathrm{Pl}-H o l a n t(f)$ is \#P-hard.

\section{Reduction from $\mathrm{PI}-\#$ CSP to $\mathrm{PI}-\# \mathrm{CSP}^{2}$}

Definition 10.1 For $k \geq 1, \ell \geq 0$ and any $\xi$, we define $E_{k}^{\ell}(\xi)=\left[1,0, \ldots, 0, \xi^{\ell}\right]$ to be a signature of arity $k$, and define $E(\xi)=\left\{E_{k}^{\ell}(\xi) \mid k \equiv \ell(\bmod 2)\right\}$. We also write $E_{k}^{\ell}$ for $E_{k}^{\ell}(\xi)$ when $\xi$ is clear from the context.

The following lemma shows that if we have a unary $[1, \xi] \in \mathcal{F}$ with $\xi \neq 0$, then either $\mathcal{F}$ is contained in one single tractable set or $\mathrm{Pl}^{-\# C^{2} P^{2}}(\mathcal{F})$ is \#P-hard. We will use this lemma for the case that $\mathcal{F}$ contains at least one nonzero signature of odd arity. The proof of this lemma also demonstrates in a simple setting the idea that will be used in the proof of Lemma 10.3.

Lemma 10.2 Let $\xi \neq 0$ and let $\mathcal{F}$ be a set of symmetric signatures containing $[1, \xi] \in \mathcal{F}$. If $\mathcal{F} \nsubseteq \mathscr{P}, \mathcal{F} \nsubseteq \mathscr{A}, \mathcal{F} \nsubseteq \mathscr{A}^{\dagger}, \mathcal{F} \nsubseteq \widehat{\mathscr{M}}$, and $\mathcal{F} \nsubseteq \widehat{\mathscr{M}^{\dagger}}$, then Pl$\# C S P^{2}(\mathcal{F})$ is \#P-hard. 
Proof Firstly, we have $E_{k}^{k}(\xi)=\partial_{[1, \xi]}^{k}(=2 k)$ of arity $k$ on the LHS in Pl$\operatorname{Holant}\left(\mathcal{E} \mathcal{Q}_{2} \mid \mathcal{F}\right) \equiv \operatorname{Pl}$-\#CSP${ }^{2}(\mathcal{F})$, for all $k \geq 1$. By a holographic transformation using $T^{-1}$, where $T=\left[\begin{array}{ll}1 & 0 \\ 0 & \xi\end{array}\right]$, we have $\left(E_{k}^{k}(\xi)\right) T^{-1}=\left(=_{k}\right)$ on the LHS, and

$$
\operatorname{Pl} \# \operatorname{CSP}(T \mathcal{F}) \leq_{T} \operatorname{Pl-Holant}\left(\mathcal{E} \mathcal{Q} \cup \mathcal{E} \mathcal{Q}_{2} T^{-1} \mid T \mathcal{F}\right) \leq_{T} \operatorname{Pl-\# CSP}(\mathcal{F}),
$$

where $\mathcal{E} \mathcal{Q}$ on LHS of the Holant instance comes from $E_{k}^{k}(\xi)$ in the second step of the reduction. If $T \mathcal{F} \nsubseteq \mathscr{P}, T \mathcal{F} \nsubseteq \mathscr{A}$ and $T \mathcal{F} \nsubseteq \widehat{\mathscr{M}}$, then $\operatorname{Pl}$-\#CSP $(T \mathcal{F})$ is \#P-hard by Theorem 9.22. Thus $\mathrm{Pl}$-\#CSP${ }^{2}(\mathcal{F})$ is \#P-hard.

Otherwise, $T \mathcal{F} \subseteq \mathscr{P}, T \mathcal{F} \subseteq \mathscr{A}$ or $T \mathcal{F} \subseteq \widehat{\mathscr{M}}$. If $T \mathcal{F} \subseteq \mathscr{P}$, then $\mathcal{F} \subseteq \mathscr{P}$. In the following, assume that $T \mathcal{F} \nsubseteq \mathscr{P}$, then $T \mathcal{F} \subseteq \mathscr{A}$ or $T \mathcal{F} \subseteq \widehat{\mathscr{M}}$.

Note that $\left[1, \xi^{2}\right] \in T \mathcal{F}$. If $\xi^{8} \neq 1$, then $\left[1, \xi^{2}\right] \notin \mathscr{A} \cup \widehat{\mathscr{M}}$. This is a contradiction. If $\xi^{4}=-1$, then $\left[1, \xi^{2}\right] \notin \widehat{\mathscr{M}}$. Thus $T \mathcal{F} \subseteq \mathscr{A}$. It follows that $\mathcal{F} \subseteq \mathscr{A}^{\dagger}$.

For $\xi^{4}=1$, if $T \mathcal{F} \subseteq \mathscr{A}$, then $\mathcal{F} \subseteq \mathscr{A}$. If $T \mathcal{F} \subseteq \widehat{\mathscr{M}}$, then either $\mathcal{F} \subseteq \widehat{\mathscr{M}}$ if $\xi^{2}=1$, or $\mathcal{F} \subseteq \widehat{\mathscr{M}}^{\dagger}$ if $\xi^{2}=-1$.

Lemma 10.2 allows us to transfer the complexity question of $\mathrm{Pl}-\# \mathrm{CSP}^{2}$ to that of $\mathrm{Pl}$-\#CSP, to which we can apply the known dichotomy (Theorem 9.22). However it requires a unary signature. We observe that if all signatures in $\mathcal{F}$ have even arities, then there is no way to construct a unary in $\operatorname{Pl}_{-\operatorname{CSP}^{2}}(\mathcal{F})$. In this case, we use the next lemma, which is similar to Lemma 10.2. It shows that if we have $[1, \xi]^{\otimes 2}$ with $\xi \neq 0$ in $\mathcal{F}$, then we can still transfer the question of Pl-\#CSP ${ }^{2}$ to that of Pl-\#CSP. It is proved using a global simulation of Pl-\#CSP by Pl-\#CSP ${ }^{2}$.

Lemma 10.3 Let $\mathcal{F}$ be a set of signatures of even arities. Suppose $[1, \xi]^{\otimes 2} \in \mathcal{F}$ for some $\xi \neq 0$. If $\mathcal{F} \nsubseteq \mathscr{P}, \mathcal{F} \nsubseteq \mathscr{A}, \mathcal{F} \nsubseteq \mathscr{A}^{\dagger}, \mathcal{F} \nsubseteq \widehat{\mathscr{M}}$ and $\mathcal{F} \nsubseteq \widehat{\mathscr{M}}^{\dagger}$, then $\mathrm{Pl} \# \mathrm{CSP}^{2}(\mathcal{F})$ is \#P-hard.

Proof Let $E_{o}(\xi)=\left\{E_{k}^{\ell}(\xi) \mid k \equiv \ell \equiv 1(\bmod 2)\right\}$ be the subset of signatures of odd arity in $E(\xi)$. Let $E_{e}(\xi)=\left\{E_{k}^{k}(\xi) \mid k \equiv 0(\bmod 2)\right\}$ be the subset of signatures $E_{k}^{\ell}(\xi) \in E(\xi)$ with even arity $k$ and $\ell=k$. Note that not all even arity signatures in $E(\xi)$ are in $E_{e}(\xi)$; in partiuclar, $k \geq 2$ for any $E_{k}^{k}(\xi) \in E_{e}(\xi)$. We will prove that $\operatorname{Pl}-H o l a n t\left(E_{o}(\xi) \cup E_{e}(\xi) \mid \mathcal{F}\right) \leq_{T} \operatorname{Pl}-\# C^{2} S^{2}(\mathcal{F})$, and the conclusion of the lemma will follow by a holographic transformation.

For the rest of this proof, we write $E_{k}^{\ell}$ for $E_{k}^{\ell}(\xi)$.

We first give an intuitive description of the proof method. For $k \geq 1$ and $\ell \geq 0$, we have all of $E_{2 k}^{2 \ell}=\partial_{[1, \xi]^{\otimes 2}}^{\ell}(=2 k+2 \ell)$ on LHS in Pl-Holant $\left(\mathcal{E} \mathcal{Q}_{2} \mid \mathcal{F}\right) \equiv \operatorname{Pl}^{\prime} \# \operatorname{CSP}^{2}(\mathcal{F})$. Given any instance $\Omega$ of Pl-Holant $\left(E_{o}(\xi) \cup E_{e}(\xi) \mid \mathcal{F}\right)$, since all signatures in $\mathcal{F}$ have even arities, the number of $E_{k}^{\ell}$ of odd arity in the LHS must be even. In each connected component of $\Omega$, we will match up all LHS $E_{k}^{\ell}$ of odd arity in pairs in a planar way, and replace each by an even arity $E_{k+1}^{\ell-1}$ connected by $[1, \xi]$, using $[1, \xi]^{\otimes 2}$ as a pair of $[1, \xi]$. Note that when one input of $E_{k+1}^{\ell-1}$ is connected to a unary $[1, \xi]$, it becomes $E_{k}^{\ell}$. Hence a pair $E_{2 v-1}^{2 u+1}$ and $E_{2 v^{\prime}-1}^{2 u^{\prime}+1}$ can be functionally replaced by a pair $E_{2 v}^{2 u}$ and $E_{2 v^{\prime}}^{2 u^{\prime}}$ that are connected by $[1, \xi]^{\otimes 2}$. When an odd arity $E_{\ell}^{k}$ is the 
signature assigned to a vertex on an edge separating two faces of the planar graph of $\Omega$, we may consider this $E_{\ell}^{k}$ as belonging to either of the two faces. If an even arity $E_{k}^{k}$ is such a signature between two faces, we can first transform it to an odd arity $E_{k+1}^{k-1}$. We will then carefully account for the number of odd arity signatures in $E_{o}(\xi)$ within each face, and argue that they can all be matched up in pairs in a planar way.

Now we give the proof. First, we may assume that the planar graph $\Omega$ is connected and is given a planar embedding, since the Holant value on $\Omega$ is the product over its connected components. The number of $E_{k}^{\ell} \in E_{o}(\xi)$ of odd arity on the LHS is even in each connected component of $\Omega$. We will match up pairs of $E_{k}^{\ell}$ of odd arity and use copies of $[1, \xi]^{\otimes 2}$ within each connected component.

Let $T$ be a spanning tree of the dual graph of $\Omega$, and we pick the external face of $\Omega$ as the root of $T$. We will process each face of $\Omega$ by going from the leaves of $T$ to the root. We prune the tree $T$ as we carry out this process; after we have dealt with one face (a leaf node in the current tree) we will delete it from the tree $T$. If on a leaf (and non-root) node of the current $T$, i.e., a face $F$ of $\Omega$, there are an even number of odd arity LHS signatures in $E_{o}(\xi)$, we replace each such $E_{k}^{\ell} \in E_{o}(\xi)$ by $E_{k+1}^{\ell-1}$, and then we connect adjacent pairs of them in clockwise order within the face $F$ with $[1, \xi]^{\otimes 2}$. This maintains planarity, and as noted above this does not change the Holant value. Then we delete this $F$ from $T$.

Suppose on the leaf node $F$ there are an odd number of odd arity LHS signatures in $E_{o}(\xi)$, and suppose $F$ is not the root of $T$. Let $F^{\prime}$ be the parent node of $F$ in $T$ and let $e=\{u, v\}$ be the edge connecting $F^{\prime}$ and $F$. Since Pl-Holant $\left(E_{o}(\xi) \cup E_{e}(\xi) \mid\right.$ $\mathcal{F}$ ) is bipartite, either $u$ or $v$ is labeled by a signature from the LHS signature set $E_{o}(\xi) \cup E_{e}(\xi)$. We have two cases.

1. It is some $E_{k}^{\ell} \in E_{o}(\xi)$. Then both $k, \ell \geq 1$ are odd. We match up the other (zero or more) odd arity LHS signatures in $E_{o}(\xi)$ within $F$ in pairs, and replace them by even arity signatures in $E(\xi)$ as above, connected with $[1, \xi]^{\otimes 2}$ in pairs while maintaining planarity as above. Then we delete this leaf node $F$ from $T$ and consider the odd arity $E_{k}^{\ell}$, which is on either $u$ or $v$, to belong to $F^{\prime}$.

2. It is some $E_{2 k}^{2 k} \in E_{e}(\xi)$, for some $k \geq 1$. On $F$ there must be some LHS odd arity $E_{s}^{t} \in E_{o}(\xi)$ since there are an odd number of them in $F$. We find clockwise the first such $E_{s}^{t}$ in $F$ starting from $E_{2 k}^{2 k}$, and replace both $E_{s}^{t}$ by $E_{s+1}^{t-1}$, and $E_{2 k}^{2 k}$ by $E_{2 k+1}^{2 k-1}$, and connect them by $[1, \xi]^{\otimes 2}$. Note that $E_{s+1}^{t-1}$ has even arity and $t-1 \geq 0$ is even, and $E_{2 k+1}^{2 k-1}$ has odd arity and $2 k-1 \geq 1$ is odd. This replacement does not change the Holant value, maintains planarity, and effectively changes the arity of $E_{2 k}^{2 k}$ between $F$ and $F^{\prime}$ to being odd. So we have transformed this case to case 1 . Thus we can match up the other odd arity LHS signatures in $E_{o}(\xi)$ within $F$ in pairs, and replace them by even arity signatures in $E(\xi)$ and connected with $[1, \xi]^{\otimes 2}$ in pairs as above. Then we delete $F$ from $T$ and consider the odd arity $E_{2 k+1}^{2 k-1}$ belonging to $F^{\prime}$.

The above process does not change the property that the total number of LHS odd arity signatures from $E_{o}(\xi)$ is even. The proof is completed by induction. After the tree has been pruned so that only the root of $T$ remains, there must be an even number 
of $E_{k}^{\ell}$ of odd arity in the external face corresponding to this root node, including those that have been transformed by its children in $T$ and considered to belong to the root node. We can pair them up just as before.

This proves the reduction

$$
\operatorname{Pl}-H o l a n t\left(E_{o}(\xi) \cup E_{e}(\xi) \mid \mathcal{F}\right) \leq_{T} \operatorname{Pl}-\# C P^{2}(\mathcal{F}) .
$$

Note that $E_{k}^{k} \in E_{o}(\xi) \cup E_{e}(\xi)$, for all $k \geq 1$. Thus we have

$$
\operatorname{Pl-Holant}\left(E_{1}^{1}, E_{2}^{2}, \ldots, E_{k}^{k}, \ldots \mid \mathcal{F}\right) \leq_{T} \operatorname{Pl}_{-\# C^{2}}^{2}(\mathcal{F}) \text {. }
$$

Then by a holographic transformation using $T^{-1}$, where $T=\left[\begin{array}{ll}1 & 0 \\ 0 & \xi\end{array}\right]$, we have

$$
\mathrm{Pl}-\# \operatorname{CSP}(T \mathcal{F}) \equiv \operatorname{Pl}-\operatorname{Holant}\left(E_{1}^{1}, E_{2}^{2}, \ldots, E_{k}^{k}, \ldots \mid \mathcal{F}\right) \leq_{T} \operatorname{Pl-\# CSP}{ }^{2}(\mathcal{F}) .
$$

The rest of the proof is essentially the same as the proof of Lemma 10.2, using the following fact: For either $\mathscr{C}=\mathscr{A}$ or $\widehat{\mathscr{M}}$, if $\left[1, \xi^{2}\right]^{\otimes 2} \in \mathscr{C}$, then $\left[1, \xi^{2}\right] \in \mathscr{C}$. For the case of $\mathscr{A}$ this follows from the form of the definition in Definition 2.5. For $\mathscr{M}$ this follows from Matchgate-Identities ([4, 10] see Theorem 4.12, page 117). The statement for $\widehat{\mathscr{M}}$ follows from the fact that holographic transformation is distributive over tensor products. We omit the details.

The next lemma shows that when we obtain $[1,0,1]^{\otimes 2}$, we can reduce a Pl-\#CSP 2 problem to a Pl-\#CSP 4 problem, when all signatures in $\mathcal{F}$ have arity divisible by 4.

Lemma 10.4 $P l$-\#CSP$P^{2}(\mathcal{F}) \leq_{T} P l$-\#CSP$P^{4}\left(\mathcal{F},[1,0,1]^{\otimes 2}\right)$, if all signatures in $\mathcal{F}$ have arity $\equiv 0(\bmod 4)$.

Proof Let $\Omega$ be an instance of $\operatorname{Pl}-\# \operatorname{CSP}^{2}(\mathcal{F})$. Since all signatures in $\mathcal{F}$ have arity $\equiv 0$ $(\bmod 4)$, the number of EQUALITIES of arity $\equiv 2(\bmod 4)$ must be even. We can connect in pairs all EQUALITIES of arity $\equiv 2(\bmod 4)$ by some copies of $[1,0,1]^{\otimes 2}$ maintaining planarity similarly as in the proof of Lemma 10.3. When two inputs of $={ }_{m+2}$ are connected to $[1,0,1]$ it becomes $\partial\left(=_{m+2}\right)=\left(=_{m}\right)$. Hence a pair $=_{4 k-2}$ and $=4 \ell-2$ can be functionally replaced by a pair $=_{4 k}$ and $=4 \ell$ that are connected by $[1,0,1]^{\otimes 2}$. The rest of the proof is the same as in Lemma 10.3 and we omit it here.

The next corollary is used in the proof of the No-Mixing theorems. We present it here since the proof uses a global simulation that is similar to Lemma 10.4.

Corollary 10.5 Suppose $f=[1, i]^{\otimes 4}+i^{r}[1,-i]^{\otimes 4}(0 \leq r \leq 3)$ and $g=$ $\left[g_{0}, \ldots, g_{2 n}\right]$ with $g_{k}=(\epsilon i)^{k}(2 n-2 k)$. Furthermore, let $\hat{g}=\left(\overline{Z^{-1}}\right)^{\otimes 2 n}$, where $Z=\left[\begin{array}{ll}1 & 1 \\ i & -i\end{array}\right]$. Then

$\operatorname{Pl}-\# \mathrm{CSP}^{2}(\hat{g}) \leq_{T} \operatorname{Pl}-\# \mathrm{CSP}^{2}(f, g)$. 
Proof Clearly $\hat{g}=[0,1,0, \ldots, 0]$ or $\hat{g}=[0, \ldots, 0,1,0]$, the perfect matching signature or its reversal. By applying Lemma 9.20 to $f=[1, i]^{\otimes 4}+i^{r}[1,-i]^{\otimes 4}$, we get $[1,0,-1]^{\otimes 2}$ on the left:

$$
\text { Pl-Holant }\left([1,0,-1]^{\otimes 2} \cup \mathcal{E} \mathcal{Q}_{2} \mid f, g\right) \leq_{T} \mathrm{Pl} \# \operatorname{CSP}^{2}(f, g) .
$$

Under a holographic transformation by $Z$, we have

$$
\text { Pl-Holant }\left([1,0,1]^{\otimes 2} \mid \hat{f}, \hat{g}\right) \leq_{T} \text { Pl-Holant }\left([1,0,-1]^{\otimes 2} \cup \mathcal{E} \mathcal{Q}_{2} \mid f, g\right),
$$

where $\hat{f}=\left(Z^{-1}\right)^{\otimes 4} f=\left[1,0,0,0, i^{r}\right]$. Note that $[1,0,-1] Z^{\otimes 2}=2[1,0,1]$, as $Z^{\mathrm{T}}\left[\begin{array}{ll}1 & 0 \\ 0 & -1\end{array}\right] Z=2\left[\begin{array}{ll}1 & 0 \\ 0 & 1\end{array}\right]$. Consider the gadget in Fig. 27. We assign $\hat{f}$ to the circle vertices and $[1,0,1]^{\otimes 2}$ the dashed subgadgets rotated appropriately so that it is equivalent to assigning $[1,0,1]$ to the square vertices. The signature of this gadget is ${ }_{4}$, for any $0 \leq r \leq 3$. Thus

$$
\text { Pl-Holant }\left([1,0,1]^{\otimes 2} \mid={ }_{4}, \hat{g}\right) \leq_{T} \text { Pl-Holant }\left([1,0,1]^{\otimes 2} \mid \hat{f}, \hat{g}\right) .
$$

In Pl-Holant $\left([1,0,1]^{\otimes 2} \mid={ }_{4}, \hat{g}\right)$, by $[1,0,1]^{\otimes 2}$ and $=4$, we can get all of $=4 k$ for $k \geq$ 1 on RHS and then move them to LHS by $[1,0,1]^{\otimes 2}$. Moreover, we have $[1,0,1]^{\otimes 2}$ on RHS by connecting two copies of $=_{4}$ by $[1,0,1]^{\otimes 2}$. Thus

$$
\operatorname{Pl}-H o l a n t\left(\mathcal{E} \mathcal{Q}_{4} \mid[1,0,1]^{\otimes 2}, \hat{g}\right) \leq_{T} \operatorname{Pl}-H o l a n t\left([1,0,1]^{\otimes 2} \mid={ }_{4}, \hat{g}\right) \text {. }
$$

Now we simulate $\mathrm{Pl}-\# \operatorname{CSP}^{2}(\hat{g})$ by Pl-Holant $\left(\mathcal{E} \mathcal{Q}_{4} \mid[1,0,1]^{\otimes 2}, \hat{g}\right)$. If $\hat{g}$ has arity $2 n \equiv 0(\bmod 4)$, then we are done by Lemma 10.4 .

If $\hat{g}$ has arity $2 n \equiv 2(\bmod 4)$, then in an instance $\Omega$ of $\operatorname{Pl}_{-\# C^{2}}(\hat{g})$, the number of occurrences of EQUALITIES of arity $\equiv 2(\bmod 4)$ has the same parity as the number of occurrences of $\hat{g}$, which could be odd. However, we observe that all entries of signatures in $\mathrm{Pl}-\# \operatorname{CSP}^{2}(\hat{g})$ are nonnegative integers. Thus the value of $\Omega$ is a nonnegative integer. Let $\Omega \uplus \Omega$ be the disjoint union of two copies of $\Omega$ as a plane graph with a common external face, then the value of $\Omega \uplus \Omega$ is the square of the value of $\Omega$. Thus computing the values on $\Omega \uplus \Omega$ and $\Omega$ are equivalent. In $\Omega \uplus \Omega$, the number of EQUALITIES of arity $\equiv 2(\bmod 4)$ is even. Now we can use the same global simulation as in Lemma 10.4, except that in the last step we may use one extra copy of $[1,0,1]^{\otimes 2}$ to connect two EQUALITIES of arity $\equiv 2(\bmod 4)$ at the two root nodes of the two spanning trees of the dual graphs of $\Omega$, if the number of occurrences of EQUALITIES of arity $\equiv 2(\bmod 4)$ in $\Omega$ is odd. Thus we have

$$
\operatorname{Pl} \# \operatorname{CSP}^{2}(\hat{g}) \leq \operatorname{Pl}-H o l a n t\left(\mathcal{E} \mathcal{Q}_{4} \mid[1,0,1]^{\otimes 2}, \hat{g}\right) .
$$

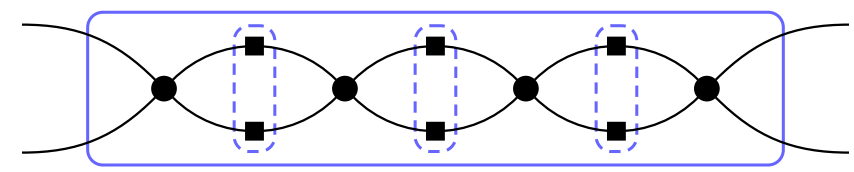

Fig. 27 Gadget used to obtain $=4$. The circle vertices are assigned $\hat{f}$ and the dashed subgadgets are assigned $[1,0,1]^{\otimes 2}$ aligned horizontally so that it is equivalent to assigning $[1,0,1]$ to the square vertices 


\section{Dichotomy Theorem when $\mathcal{F}$ Contains an Odd Arity Signature}

In this section, we give a dichotomy theorem for $\operatorname{Pl}-\# \operatorname{CSP}^{2}(\mathcal{F})$, where $\mathcal{F}$ includes at least one nonzero signature $f$ that has odd arity.

The next result applies a domain pairing technique used in the proof of Lemma 6.2 in [26].

Lemma 11.1 Let $x, y \in \mathbb{C}$ and $f=[x, 0, y, 0]$. If $y \neq 0$ and $x^{4} \neq y^{4}$, then $P l$-\#CSP $P^{2}(f)$ is \#P-hard.

Proof We reduce from $\mathrm{Pl}-\# \mathrm{CSP}\left(\left[x^{2}, y^{2}, y^{2}\right]\right)$ to $\mathrm{Pl}-\# \mathrm{CSP}^{2}(f)$. Since $\mathrm{Pl}$ \#CSP $\left(\left[x^{2}, y^{2}, y^{2}\right]\right)$ is \#P-hard when $y \neq 0$ and $x^{4} \neq y^{4}$ by Theorem 9.21 , this shows that $\mathrm{Pl}$ \#CSP${ }^{2}(f)$ is also \#P-hard.

An instance of $\mathrm{Pl}$-\#CSP $\left(\left[x^{2}, y^{2}, y^{2}\right]\right)$ is a signature grid $\Omega$ with underlying graph $G=(U, V, E)$, where $G$ is bipartite and planar, and every vertex in $U$ has degree 2 . We replace every vertex in $V$ of degree $k$ (which is assigned $=_{k} \in \mathcal{E} \mathcal{Q}$ ) with a vertex of degree $2 k$, and bundle two adjacent variables to form $k$ bundles of 2 edges each. The $k$ bundles correspond to the $k$ incident edges of the original vertex with degree $k$. We assign $=2 k$ to the new vertices of degree $2 k$.

If the inputs to these equality signatures are restricted to $\{(0,0),(1,1)\}$ on each bundle, then these equality signatures take value 1 on $((0,0), \ldots,(0,0))$ and $((1,1), \ldots,(1,1))$ and take value 0 elsewhere. Thus, if we restrict the domain to $\{(0,0),(1,1)\}$, it is the equality signature $=_{k}$.

To simulate $\left[x^{2}, y^{2}, y^{2}\right]$, we connect two copies of $f=[x, 0, y, 0]$ by a single edge as shown in Fig. 28 to form a gadget with signature

$$
h\left(a_{1}, a_{2}, b_{1}, b_{2}\right)=\sum_{c=0,1} f\left(a_{1}, b_{1}, c\right) f\left(a_{2}, b_{2}, c\right) .
$$

We replace every (degree 2) vertex in $U$ (which is assigned $\left[x^{2}, y^{2}, y^{2}\right]$ ) by a degree 4 vertex assigned $h$, where the variables of $h$ are bundled as $\left(a_{1}, a_{2}\right)$ and $\left(b_{1}, b_{2}\right)$.

The vertices in this new graph $G^{\prime}$ are connected as in the original graph $G$, except that every original edge is replaced by two edges that connect to the same side of the gadget in Fig. 28. Notice that $h$ is only connected by $\left(a_{1}, a_{2}\right)$ and $\left(b_{1}, b_{2}\right)$ to some bundle of two incident edges of an equality signature. Since this equality signature enforces that the value on each bundle is either $(0,0)$ or $(1,1)$, we only need to consider the restriction of $h$ to the domain $\{(0,0),(1,1)\}$. On this domain,

Fig. 28 Gadget designed for the paired domain. Both vertices are assigned $[x, 0, y, 0]$

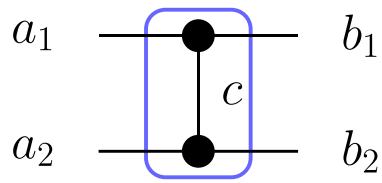


$h=\left[x^{2}, y^{2}, y^{2}\right]$ is a symmetric signature of arity 2 . Therefore, the signature grid $\Omega^{\prime}$ with underlying graph $G^{\prime}$ has the same Holant value as the original signature grid $\Omega$.

The following lemma is a dichotomy for $\mathrm{Pl}_{-} \mathrm{CSP}^{2}(f)$ where $f$ is a symmetric ternary signature.

Lemma 11.2 Let $f$ be a symmetric signature of arity 3 , then $\mathrm{Pl}$-\#CSP${ }^{2}(f)$ is \#P-hard unless $f \in \mathscr{P} \cup \widetilde{\mathscr{A}} \cup \widetilde{\mathscr{M}}$.

Proof Let $f=\left[f_{0}, f_{1}, f_{2}, f_{3}\right]$. If $f$ satisfies the parity constraints, then $f=$ $\left[f_{0}, 0, f_{2}, 0\right]$ or $f=\left[0, f_{1}, 0, f_{3}\right]$.

For $f=\left[f_{0}, 0, f_{2}, 0\right]$, if $f_{2}=0$, then $f \in \mathscr{P}$. If $f_{0}^{2}=f_{2}^{2}$, then $f \in \mathscr{A}$. If $f_{0}^{2}=-f_{2}^{2}$, then $f \in \mathscr{A}^{\dagger}$. Otherwise, we have $f_{2} \neq 0$ and $f_{0}^{4} \neq f_{2}^{4}$. Thus Pl-\#CSP ${ }^{2}(f)$ is \#P-hard by Lemma 11.1. For $f=\left[0, f_{1}, 0, f_{3}\right]$, the proof follows from a holographic transformation using $\left[\begin{array}{ll}0 & 1 \\ 1 & 0\end{array}\right]$.

In the following, assume that $f$ does not satisfy the parity constraints. Firstly, we have $\partial(f)=\left[f_{0}+f_{2}, f_{1}+f_{3}\right]$.

- $\quad$ For $\left(f_{0}+f_{2}\right)\left(f_{1}+f_{3}\right) \neq 0$, we are done by Lemma 10.2.

- For $f_{0}+f_{2}=f_{1}+f_{3}=0, f=\left[f_{0}, f_{1},-f_{0},-f_{1}\right]$. Since $f$ does not satisfy the parity constraints, we have $f_{0} f_{1} \neq 0$. If $f_{0}^{2}=f_{1}^{2}$, then $f \in \mathscr{A}$.

Otherwise, we have $\partial_{f}\left(=_{4}\right)=\left[f_{0},-f_{1}\right]$ on LHS and $\partial_{\left[f_{0},-f_{1}\right]}(f)=\left[f_{0}^{2}-\right.$ $\left.f_{1}^{2}, 2 f_{0} f_{1}, f_{1}^{2}-f_{0}^{2}\right]$ on RHS. Moreover, we have $\partial_{\left[f_{0}^{2}-f_{1}^{2}, 2 f_{0} f_{1}, f_{1}^{2}-f_{0}^{2}\right]}(=4)=$ $\left(f_{0}^{2}-f_{1}^{2}\right)[1,0,-1]$ on LHS, where $f_{0}^{2}-f_{1}^{2} \neq 0$. So we have $\partial_{[1,0,-1]}(f)=$ $2\left[f_{0}, f_{1}\right]$ on RHS. Then we are done by Lemma 10.2 and $f_{0} f_{1} \neq 0$.

- For $f_{0}+f_{2} \neq 0, f_{1}+f_{3}=0$, we have $f_{1}=-f_{3} \neq 0$ since $f$ does not satisfy the parity constraints. Note that we have $\partial(f)=\left(f_{0}+f_{2}\right)[1,0]$ in RHS, so we have $\partial_{[1,0]}^{2}(f)=\left[f_{0}, f_{1}\right]$ in RHS. If $f_{0} \neq 0$, then we are done by Lemma 10.2. If $f_{0}=0$, then $f_{2} \neq 0$ since $f_{0}+f_{2} \neq 0$. Note that we have $f_{1}[0,1]$ and $f_{2}[1,0]$ now. Thus we have $\partial_{[1,0]}\left[\partial_{[0,1]}(f)\right]=\left[f_{1}, f_{2}\right]$. Then we are done by Lemma 10.2.

- For $f_{0}+f_{2}=0, f_{1}+f_{3} \neq 0$, the proof follows from a holographic transformation using $\left[\begin{array}{ll}0 & 1 \\ 1 & 0\end{array}\right]$.

The next lemma shows that if we have an odd arity signature in $\widetilde{\mathscr{M}} \backslash(\mathscr{P} \cup \tilde{\mathscr{A}})$, then we can prove Theorem 9.2 directly. The key point is that we can use such a signature to get a unary $[1, \xi]$ with $\xi \neq 0$.

Lemma 11.3 Let $\mathcal{F}$ be a symmetric signature set and suppose $f \in \mathcal{F}$ has odd arity.

- If $f \in \widehat{\mathscr{M} \backslash} \backslash(\mathscr{P} \cup \widetilde{\mathscr{A}})$, then either $\mathcal{F} \subseteq \widehat{\mathscr{M}}$ or $\operatorname{Pl}_{-} \operatorname{CSP}^{2}(\mathcal{F})$ is $\# P$-hard.

- If $f \in \widehat{\mathscr{M}}^{\dagger} \backslash(\mathscr{P} \cup \mathscr{\mathscr { A }})$, then either $\mathcal{F} \subseteq \widehat{\mathscr{M}}^{\dagger}$ or $\mathrm{Pl}_{-\mathrm{CSP}^{2}}(\mathcal{F})$ is \#P-hard. 
Proof We will use our calculus with the derivative operator $\partial$. Firstly, we prove the lemma for $f \in \widehat{\mathscr{M}} \backslash(\mathscr{P} \cup \widetilde{\mathscr{A}})$. We already have $\mathcal{F} \nsubseteq \mathscr{P}, \mathcal{F} \nsubseteq \mathscr{A}, \mathcal{F} \nsubseteq \mathscr{A}^{\dagger}$ by the presence of $f$, and $\mathcal{F} \nsubseteq \widehat{\mathscr{M}}$ by Corollary 9.13. If we can construct a unary $[a, b]$ with $a b \neq 0$, then we can finish the proof by Lemma 10.2.

As $f \notin \mathscr{P}$ and has odd arity, its arity $n \geq 3$. By Lemma 9.14, the signature $f \in \widehat{\mathscr{M} \backslash} \backslash(\mathscr{P} \cup \widetilde{\mathscr{A}})$ can take one of the following two forms (see the Calculus after Definition 9.4):

- For $f=[s, t]^{\otimes n} \pm[t, s]^{\otimes n}$, where $n \geq 3$ is odd, we have $s t \neq 0$ and $s^{4} \neq t^{4}$. Thus we have $\partial^{\frac{n-1}{2}}(f)=\left(s^{2}+t^{2}\right)^{\frac{n-1}{2}}([s, t] \pm[t, s])=\left(s^{2}+t^{2}\right)^{\frac{n-1}{2}}(s \pm t)[1, \pm 1]$, a nonzero multiple of $[1, \pm 1]$. So we are done by Lemma 10.2.

- For $f_{k}=\lambda \epsilon^{k}(n-2 k)$, we have $\partial^{\frac{n-1}{2}}(f)=2^{\frac{n-1}{2}} \lambda[1,-\epsilon]$ and we are done by Lemma 10.2.

Similarly, for $f \in \widehat{\mathscr{M}}^{\dagger} \backslash(\mathscr{P} \cup \widetilde{\mathscr{A}})$, we just need to construct a unary $[a, b]$ with $a b \neq 0$.

- $\quad$ For $f=[s, t i]^{\otimes n} \pm[t, s i]^{\otimes n}$, we have $\partial^{\frac{n-1}{2}}(f)=\left(s^{2}-t^{2}\right)^{\frac{n-1}{2}}[s, t i] \pm\left(t^{2}-\right.$ $\left.s^{2}\right)^{\frac{n-1}{2}}[t, s i]=\left(s^{2}-t^{2}\right)^{\frac{n-1}{2}}(s \pm t)[1, \pm i]$. By Lemma 9.14, we have $s t \neq 0$ and $s^{4} \neq t^{4}$, and so this is a nonzero multiple of $[1, \pm i]$. So we are done by Lemma 10.2.

- For $f_{k}=\lambda(\epsilon i)^{k}(n-2 k)$, if $n \equiv 1(\bmod 4)$, we have $\partial_{=4}^{\frac{n-1}{4}}(f)=2^{\frac{n-1}{4}} \lambda[1,-\epsilon i]$ and we are done by Lemma 10.2. If $n \equiv 3(\bmod 4)$, we have $\partial\left[\partial_{=4}^{\frac{n-3}{4}}(f)\right]=$ $2^{\frac{n+5}{4}} \lambda[1, \epsilon i]$ and we are done by Lemma 10.2 .

We remark that the use of $\partial_{=4}$ instead of just $\partial$ in this proof is necessary, because $\partial^{2}(f)=0$ when $f_{k}=\lambda(\epsilon i)^{k}(n-2 k)$ and $n \geq 5$. One may also suppose that the case for $\widehat{\mathscr{M}} \backslash(\mathscr{P} \cup \widetilde{\mathscr{A}})$ can be reduced to the case for $\widehat{\mathscr{M} \backslash}(\mathscr{P} \cup \widetilde{\mathscr{A}})$ by the transformation $T=\left[\begin{array}{ll}1 & 0 \\ 0 & i\end{array}\right]$. While $T$ transforms $\widehat{\mathscr{M}}^{\dagger}$ to $\widehat{\mathscr{M}}$, and keeps $\mathscr{P}$ and $\tilde{\mathscr{A}}$ invariant, this transformation does not keep $\mathcal{E} \mathcal{Q}_{2}$ invariant. In fact $[1,0,1] T_{\sim}^{\otimes 2}=[1,0,-1] \notin$ $\mathcal{E} \mathcal{Q}_{2}$. Therefore we need to handle the proof for $\widehat{\mathscr{M}}^{\dagger} \backslash(\mathscr{P} \cup \mathscr{\mathscr { A }})$ separately.

By definitions of $\mathscr{P}$ and $\widetilde{\mathscr{A}}$, we have the following simple lemma.

Lemma 11.4 Assume that $f$ satisfies the parity constraints, then $f \in \mathscr{P} \cup \tilde{\mathscr{A}}$ iff $f$ belongs to the following set, up to a scalar factor $\left\{[1,0]^{\otimes n},[0,1]^{\otimes n},[1,0]^{\otimes 2 n}+t[0,1]^{\otimes 2 n},[1, \rho]^{\otimes n} \pm[1,-\rho]^{\otimes n},[1, \alpha]^{\otimes n} \pm[1,-\alpha]^{\otimes n} \mid t \neq 0, n \geq 1\right\}$.

Proof Note that $f$ satisfies the parity constraints. Then by Definition $2.6, f \in \mathscr{P}$ iff $f$ belongs to

$$
\left\{[1,0]^{\otimes n},[0,1]^{\otimes n},[1,0]^{\otimes 2 n}+t[0,1]^{\otimes 2 n} \mid t \neq 0, n \geq 1\right\}
$$

up to a scalar factor. Moreover, by the lists after Definition $2.5, f \in \mathscr{A} \backslash \mathscr{P}$ satisfies the parity constraints iff $f$ has the form $[1, \rho]^{\otimes n} \pm[1,-\rho]^{\otimes n}$ up to a scalar factor. 
Then after the holographic transformation using $\left[\begin{array}{ll}1 & 0 \\ 0 & \alpha\end{array}\right], f \in \mathscr{A}^{\dagger} \backslash \mathscr{P}$ iff $f$ has the form $[1, \alpha]^{\otimes n} \pm[1,-\alpha]^{\otimes n}$ up to a scalar factor. This finishes the proof.

The next lemma shows that if we have a nonzero odd arity signature $f \in \mathscr{P} \cup \tilde{\mathscr{A}}$ that does not satisfy the parity constraints, then we can obtain a unary $[a, b]$ with $a b \neq 0$. Note that if we have a unary $[a, b]$ with $a b \neq 0$, then we can apply Lemma 10.2.

Lemma 11.5 If $f \in \mathscr{P} \cup \tilde{\mathscr{A}}$ has odd arity and does not satisfy the parity constraints, then we can construct a unary $[a, b]$ with $a b \neq 0$ in $\mathrm{Pl}_{-1} \mathrm{CSP}^{2}(f)$.

Proof Let $f$ have arity $2 n+1, n \geq 0$. Not satisfying the parity constraints implies that $f$ is not identically 0 . Up to a nonzero factor, $f$ has one of the following forms. If $f \in \mathscr{P}$, then $f=[a, b]^{\otimes 2 n+1}$ with $a b \neq 0$ or $f=[1,0, \ldots, 0, x]$ with $x \neq 0$.

- If $f=[1,0, \ldots, 0, x], x \neq 0$, then $\partial^{n}(f)=[1, x]$.

- If $f=[a, b]^{\otimes 2 n+1}, a^{2}+b^{2} \neq 0$, then $\partial^{n}(f)=\left(a^{2}+b^{2}\right)^{n}[a, b]$.

- For $f=[1, \pm i]^{\otimes 2 n+1}$, if $n$ is even, then $\partial_{=}^{\frac{n}{2}}(f)=2^{\frac{n}{2}}[1, \pm i]$. If $n$ is odd, then we have $\partial_{f}(=2 n+2)=[1, \mp i]$ on LHS and we have $\partial_{[1, \mp i]}^{2 n}(f)=2^{2 n}[1, \pm i]$ on RHS.

For $f \in \tilde{\mathscr{A}} \backslash \mathscr{P}$, we have $f=[1, \rho]^{\otimes 2 n+1} \pm i[1,-\rho]^{\otimes 2 n+1}$ or $f=[1, \alpha]^{\otimes 2 n+1} \pm$ $i[1,-\alpha]^{\otimes 2 n+1}$.

- If $f=[1, \alpha]^{\otimes 2 n+1} \pm i[1,-\alpha]^{\otimes 2 n+1}$ or $[1, \rho]^{\otimes 2 n+1} \pm i[1,-\rho]^{\otimes 2 n+1}$ with $\rho^{2}=1$, then $\partial^{n}(f)=\left(1+\alpha^{2}\right)^{n}[1 \pm i,(1 \mp i) \alpha]$ or $\left(1+\rho^{2}\right)^{n}[1 \pm i,(1 \mp i) \rho]$.

- For $f=[1, \rho]^{\otimes 2 n+1} \pm i[1,-\rho]^{\otimes 2 n+1}$ with $\rho^{2}=-1$, and if $n$ is even, then we have $\partial_{2_{4}^{\frac{n}{2}}}(f)=2^{\frac{n}{2}}[1 \pm i,(1 \mp i) \rho]$. If $n$ is odd, then $2 n+1 \equiv 3(\bmod 4)$, and $( \pm \rho)^{2 n+1}= \pm \rho^{3}=\mp \rho$, by $\rho^{2}=-1$. Then we have $\partial_{f}(=2 n+2)=$ $\left[1, \rho^{2 n+1}\right] \pm i\left[1,(-\rho)^{2 n+1}\right]=[1,-\rho] \pm i[1, \rho]=(1 \pm i)[1, \pm i \rho]$ on LHS. Note that $\left(\frac{1 \mp i}{1 \pm i}\right)^{2 n}=(\mp i)^{2 n}=(-1)^{n}=-1$ since $n$ is odd.

Then we have $\partial_{[1, \pm i \rho]}^{2 n}(f)=(1 \mp i)^{2 n}[1, \rho] \pm i(1 \pm i)^{2 n}[1,-\rho]=(1 \pm$ $i)^{2 n}\left\{\left(\frac{1 \mp i}{1 \pm i}\right)^{2 n}[1, \rho] \pm i[1,-\rho]\right\}=(1 \pm i)^{2 n}\{-[1, \rho] \pm i[1,-\rho]\}=(1 \pm i)^{2 n}[-1 \pm$ $i,-\rho(1 \pm i)]$.

If a signature $f$ satisfies the parity constraints, then there is no way to construct $[a, b]$ with $a b \neq 0$ from $f$. In fact in $\mathrm{Pl}$-\#CSP${ }^{2}$ using $f$, the signature of any $\{f\}$-gate will also satisfy the parity constraints, and in particular a unary signature can only be a multiple of $[1,0]$ or $[0,1]$. The next lemma shows that if we have a nonzero odd arity signature $f \in \mathscr{P} \cup \mathscr{A}$ that satisfies the parity constraints, then we can obtain $[1,0]$ or $[0,1]$. We also remark that in $\mathrm{Pl}-\# \mathrm{CSP}^{2}$ using signatures of even arity one can only produce signatures of even arity, and thus no unary signatures. 
Lemma 11.6 If a nonzero $f \in \mathscr{P} \cup \tilde{\mathscr{A}}$ has odd arity and satisfies the parity constraints, then we can construct a unary $[1,0]$ or $[0,1]$ in $P l-\# C S P^{2}(f)$.

Proof By Lemma 11.4, any nonzero $f$ of odd arity belongs to the following set, up to a nonzero factor,

$\left.\left.\left\{[1,0]^{\otimes 2 n+1},[0,1]^{\otimes 2 n+1},[1, \rho]^{\otimes 2 n+1} \pm[1,-\rho]^{\otimes 2 n+1}\right],[1, \alpha]^{\otimes 2 n+1} \pm[1,-\alpha]^{\otimes 2 n+1}\right] \mid n \geq 0\right\}$.

For $f=[1,0]^{\otimes 2 n+1},[0,1]^{\otimes 2 n+1},[1, \alpha]^{\otimes 2 n+1} \pm[1,-\alpha]^{\otimes 2 n+1}$ or $[1, \rho]^{\otimes 2 n+1} \pm$ $[1,-\rho]^{\otimes 2 n+1}$ with $\rho^{2}=1, \partial^{n}(f)=[1,0],[0,1],\left(1+\alpha^{2}\right)^{n}[1 \pm 1,(1 \mp 1) \alpha]$ or $\left(1+\rho^{2}\right)^{n}[1 \pm 1,(1 \mp 1) \rho]$ respectively, all of which are nonzero multiples of $[1,0]$ or $[0,1]$.

For $f=[1, \rho]^{\otimes 2 n+1} \pm[1,-\rho]^{\otimes 2 n+1}$, with $\rho^{2}=-1$, if $2 n+1 \equiv 1(\bmod 4)$, then $\partial^{\frac{n}{2}}(f)=2^{\frac{n}{2}}[1 \pm 1,(1 \mp 1) \rho]$, a nonzero multiple of $[1,0]$ or $[0,1]$. If $2 n+1 \equiv 3$ $(\bmod 4)$, then $( \pm \rho)^{2 n+1}=\mp \rho$. If we write $f=\left[f_{0}, f_{1}, \ldots, f_{2 n+1}\right]$, then exactly one of $f_{0}$ and $f_{2 n+1}$ is nonzero. We have the unary $u=\partial_{f}(=2 n+2)=\left[f_{0}, f_{2 n+1}\right]$ in LHS, a nonzero multiple of $[1,0]$ or $[0,1]$. Then we get $\partial_{u}^{2 n}(f)$ in RHS, also a nonzero multiple of $[1,0]$ or $[0,1]$.

The next lemma shows that if we already have $[1,0]$ or $[0,1]$ and also a signature $f$ of any arity that does not satisfy the parity constraints, then we can construct a unary $[a, b]$ with $a b \neq 0$.

Lemma 11.7 If $f$ does not satisfy the parity constraints, then we can construct a unary $[a, b]$ with $a b \neq 0$ in $\mathrm{Pl}-\# \operatorname{CSP}^{2}([1,0], f)$ or $\mathrm{Pl}_{-\# C^{2}}([0,1], f)$.

Proof We prove the lemma for $\mathrm{Pl}_{-} \mathrm{CSSP}^{2}([1,0], f)$. The proof for the other case follows from a holographic transformation by $\left[\begin{array}{ll}0 & 1 \\ 1 & 0\end{array}\right]$.

Let $f=\left[f_{0}, f_{1}, \ldots, f_{n}\right]$. Since $f$ does not satisfy the parity constraints, there exist $0 \leq i<j \leq n$ such that $\left[f_{i}, f_{i+1}, \ldots, f_{j-1}, f_{j}\right]=\left[f_{i}, 0, \ldots, 0, f_{j}\right]$, where $f_{i} f_{j} \neq 0$ and $j-i$ is odd. We can get both $f^{\prime}=\partial_{[1,0]}^{n-i}=\left[f_{0}, f_{1}, \ldots, f_{i}\right]$ and $f^{\prime \prime}=\partial_{[1,0]}^{n-j}=\left[f_{0}, f_{1}, \ldots, f_{j}\right]$ on RHS. Either $i$ or $j$ is odd. And so we have either $=_{i+1}$ or $=j+1$, and we can get either $\partial_{f^{\prime}}\left(=_{i+1}\right)=\left[f_{0}, f_{i}\right]$ or $\partial_{f^{\prime \prime}}\left(={ }_{j+1}\right)=\left[f_{0}, f_{j}\right]$ on LHS. Without loss of generality, assume that we have $\left[f_{0}, f_{i}\right]$ on LHS.

If $f_{0}=0$, then we have $[0,1]$ on LHS and $f^{\prime \prime \prime}=\partial^{\frac{j-i-1}{2}}\left(\partial_{[0,1]}^{i}\left(f^{\prime \prime}\right)\right)=$ $\partial^{\frac{j-i-1}{2}}\left(\left[f_{i}, 0, \ldots, 0, f_{j}\right]\right)=\left[f_{i}, f_{j}\right]$ on RHS, and we are done.

If $f_{0} \neq 0$, let $m=\min _{1 \leq k \leq n}\left\{k \mid f_{k} \neq 0\right\}$. (As $j>0$ and $f_{j} \neq 0$, this $m$ is well-defined.) Then $f^{(4)}=\partial_{[1,0]}^{n-m}(f)=\left[f_{0}, 0, \ldots, 0, f_{m}\right]$. Moreover, we have $\partial_{\left[f_{0}, f_{i}\right]}^{m-1}\left(f^{(4)}\right)=\left[f_{0}^{m}, f_{i}^{m-1} f_{m}\right]$ with two nonzero entries.

The next lemma assumes the presence of a non-degenerate binary GEN-EQ. The conclusion is about a transformed signature but still in the $\mathrm{Pl}_{-1} \mathrm{CSP}^{2}$ setting. 
Lemma 11.8 For any $x \neq 0$ and any signature $f$ of arity $2 n$, let $\hat{f}^{x}=$ $\left[\begin{array}{ll}1 & 0 \\ 0 & x^{-\frac{1}{2}}\end{array}\right]^{\otimes 2 n} f$. Then $P l$-\#CSP$P^{2}\left(\hat{f}^{x}\right) \leq_{T} P l$-\#CSP$P^{2}(f,[1,0, x])$.

Proof After a holographic transformation by $\left[\begin{array}{ll}1 & 0 \\ 0 & x^{\frac{1}{2}}\end{array}\right]$, we have

$$
\operatorname{Pl-\# CSP}([1,0, x], f) \equiv_{T} \operatorname{Pl-Holant}\left([1,0, x],\left[1,0,0,0, x^{2}\right], \cdots \mid[1,0,1], \hat{f}^{x}\right) .
$$

If $x$ is a root of unity, then there exists a $t \geq 1$ such that $x^{t}=1$. Thus we have $=2 k t$ for all $k \geq 1$ on LHS. Moreover, we have $=_{2 k}$ by $\partial^{k(t-1)}(=2 k t)$ on LHS for all $k \geq 1$. Thus we are done.

If $x$ is not a root of unity, then we have $\partial^{d-2}\left(E_{2 d}^{d}(x)\right)=\left[1,0,0,0, x^{d}\right]$ of arity 4 on LHS for all $d \geq 2$, where $E_{2 d}^{d}(x)=\left[1,0, \ldots, 0, x^{d}\right]$ has arity $2 d$. Thus we can get $[1,0,0,0,1]$ on LHS by interpolation. Then we can get all of $=2 k$ on LHS since we have $[1,0,1]$ on RHS.

Lemma 11.9 Suppose either $f=[1, \rho]^{\otimes 3} \pm[1,-\rho]^{\otimes 3}$ or $f=[1, \alpha]^{\otimes 3} \pm[1,-\alpha]^{\otimes 3}$, and let $h=[1,0, x]$. If $x^{4} \notin\{0,1\}$, then $\mathrm{Pl}-\# \mathrm{CSP}^{2}(f, h)$ is \#P-hard.

Proof We prove the lemma for $f=[1, \rho]^{\otimes 3} \pm[1,-\rho]^{\otimes 3}$. The proof for $f=$ $[1, \alpha]^{\otimes 3} \pm[1,-\alpha]^{\otimes 3}$ is similar and we omit it here.

Let $\hat{f}=\left[1, x^{-\frac{1}{2}} \rho\right]^{\otimes 3} \pm\left[1,-x^{-\frac{1}{2}} \rho\right]^{\otimes 3}$, then $\operatorname{Pl}^{8} \# \operatorname{CSP}^{2}(\hat{f}) \leq \operatorname{Pl}-\# \operatorname{CSP}^{2}(f, h)$ by Lemma 11.8. $\hat{f}$ satisfies a second-order recurrence with eigenvalues $\pm x^{-\frac{1}{2}} \rho$ with sum 0 and product $-\rho^{2} / x$. Hence $\hat{f}$ has type $\left\langle-\rho^{2} / x, 0,1\right\rangle$. Moreover, the secondorder recurrence relation is unique up to a scalar since $\hat{f}$ is non-degenerate and has arity 3 . By $\left(x^{-1} \rho^{2}\right)^{4} \neq 1$, we have $\hat{f} \notin \mathscr{P} \cup \widetilde{\mathscr{A}} \cup \widetilde{\mathscr{M}}$ by Lemma 9.11 . So $\mathrm{Pl}$-\#CSP${ }^{2}(\hat{f})$ is \#P-hard by Lemma 11.2. Thus $\mathrm{Pl}^{-\# C^{2}}{ }^{2}(f, h)$ is \#P-hard.

Lemma 11.10 Let $f=[1, \rho]^{\otimes 3} \pm[1,-\rho]^{\otimes 3}$ and $g=[1, \alpha]^{\otimes 3} \pm[1,-\alpha]^{\otimes 3}$. Then $\mathrm{Pl}$-\#CSP $\mathrm{CS}^{2}(f, g)$ is \#P-hard.

Proof Consider the gadget in Fig. 29. We assign $f$ to the circle vertices and $g$ to the triangle vertices. Let $h$ be the signature of this gadget.

- If $f=[1, \rho]^{\otimes 3}+[1,-\rho]^{\otimes 3}$ and $g=[1, \alpha]^{\otimes 3}+[1,-\alpha]^{\otimes 3}$, then $h=$ $32\left[\rho^{2} \alpha^{2}, 0,-2\right]$.

- If $f=[1, \rho]^{\otimes 3}-[1,-\rho]^{\otimes 3}$ and $g=[1, \alpha]^{\otimes 3}+[1,-\alpha]^{\otimes 3}$, then $h=$ $32 \rho^{2}\left[-2,0, \rho^{2} \alpha^{2}\right]$.

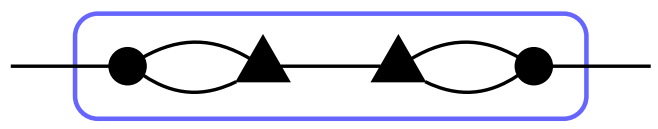

Fig. 29 Gadget used to obtain a signature of the form $[a, 0, b]$ with $|a| \neq|b|$. The circle vertices are assigned $f$ and the triangle vertices are assigned $g$ 
- If $f=[1, \rho]^{\otimes 3}+[1,-\rho]^{\otimes 3}$ and $g=[1, \alpha]^{\otimes 3}-[1,-\alpha]^{\otimes 3}$, then $h=$ $32 \alpha^{2}\left[\rho^{2} \alpha^{2}, 0,2\right]$.

- If $f=[1, \rho]^{\otimes 3}-[1,-\rho]^{\otimes 3}$ and $g=[1, \alpha]^{\otimes 3}-[1,-\alpha]^{\otimes 3}$, then $h=$ $32 \rho^{2} \alpha^{2}\left[2,0, \rho^{2} \alpha^{2}\right]$.

Note that both $f$ and $g$ satisfy the parity constraints, and thus $h$ also satisfies them. Hence, e.g., in the first case, $f=2\left[1,0, \rho^{2}, 0\right]$ and $g=2\left[1,0, \alpha^{2}, 0\right]$, we only need to calculate $h_{0}$ and $h_{2}$, since $h_{1}=0$ by parity. In fact the left half of Fig. 29, connecting $f$ to $g$, also satisfies the parity constraints and has the signature $4[1+$ $\left.\rho^{2} \alpha^{2}, 0,2 \rho^{2} \alpha^{2}\right]$, and thus $h=16\left[\left(1+\rho^{2} \alpha^{2}\right)^{2}, 0,4\left(\rho^{2} \alpha^{2}\right)^{2}\right]=32\left[\rho^{2} \alpha^{2}, 0,-2\right]$ (recall that $\rho^{4}=1$ and $\alpha^{4}=-1$ ).

Since $|\alpha \rho|=1 \neq 2$, we are done by Lemma 11.9.

Recall the notations $S_{1}, \ldots, S_{5}$ introduced in (9.12). We have

$$
\bigcup_{k=1}^{5} S_{k}=\mathscr{P} \cup \tilde{\mathscr{A}} \cup \widetilde{\mathscr{M}}=\mathscr{P} \cup \mathscr{A} \cup \mathscr{A}^{\dagger} \cup \widehat{\mathscr{M}} \cup \widehat{\mathscr{M}}{ }^{\dagger} .
$$

Now we can prove a conditional No-Mixing theorem for Pl-\#CSP ${ }^{2}$ when a set of signatures $\mathcal{F}$ is assumed to consist of only tractable signatures and has at least one nonzero signature of odd arity.

Theorem 11.11 Let $\mathcal{F} \subseteq \bigcup_{k=1}^{5} S_{k}$ be a set of symmetric signatures that includes at least one nonzero signature of odd arity. If $\mathcal{F} \nsubseteq S_{k}$ for all $1 \leq k \leq 5$, then $\mathrm{Pl} \# \operatorname{CSP}^{2}(\mathcal{F})$ is \#P-hard.

Proof If $\mathcal{F}$ contains a signature of odd arity in $\widetilde{\mathscr{M}} \backslash(\mathscr{P} \cup \widetilde{\mathscr{A}})$, then we are done by Lemma 11.3. Thus we can assume that $\mathcal{F}$ contains at least one nonzero signature of odd arity $f \in \mathscr{P} \cup \widetilde{\mathscr{A}}$.

By Lemma 11.5, if $f$ does not satisfy the parity constraints, then we have a unary $[a, b]$ with $a b \neq 0$ and we are done by Lemma 10.2. Otherwise, we have [1,0] or $[0,1]$ by Lemma 11.6. If there exists a signature in $\mathcal{F}$ that does not satisfy the parity constraints, then we can obtain a unary $[a, b]$ with $a b \neq 0$ by Lemma 11.7. Thus we are done by Lemma 10.2 .

Now we can assume that $\mathcal{F}$ includes a nonzero odd arity signature $f \in \mathscr{P} \cup \tilde{\mathscr{A}}$ and all signatures in $\mathcal{F}$ satisfy the parity constraints. Thus $\mathcal{F} \cap(\widetilde{\mathscr{M}} \backslash(\mathscr{P} \cup \tilde{\mathscr{A}}))=\emptyset$ by Corollary 9.15. So we have $\mathcal{F} \subseteq \mathscr{P} \cup \widetilde{\mathscr{A}}$, i.e., $\mathcal{F} \subseteq \bigcup_{k=3}^{5} S_{k}$. Then by Lemma 11.4, we have, up to scalar multiples,

$$
\mathcal{F} \subseteq\left\{\begin{array}{l}
{[1,0]^{\otimes n}, \quad[0,1]^{\otimes n}, \quad[1,0]^{\otimes 2 n}+t[0,1]^{\otimes 2 n},} \\
{[1, \rho]^{\otimes n} \pm[1,-\rho]^{\otimes n}, \quad[1, \alpha]^{\otimes n} \pm[1,-\alpha]^{\otimes n}}
\end{array} \mid t \neq 0 \text { and } n \geq 1\right\} .
$$


Note that the following signatures are all in $\bigcap_{k=3}^{5} S_{k}$ (see Fig. 24): For $n \geq 1$,

$$
\begin{aligned}
& {[1,0]^{\otimes n} \quad \text { and } \quad[0,1]^{\otimes n},} \\
& {[1,0]^{\otimes 2 n}+t[0,1]^{\otimes 2 n} \quad \text { with } t^{4}=1,} \\
& {[1, \rho]^{\otimes m} \pm[1,-\rho]^{\otimes m} \quad \text { and } \quad[1, \alpha]^{\otimes \ell} \pm[1,-\alpha]^{\otimes \ell} \quad \text { with } \quad 1 \leq m, \ell \leq 2 .}
\end{aligned}
$$

Let

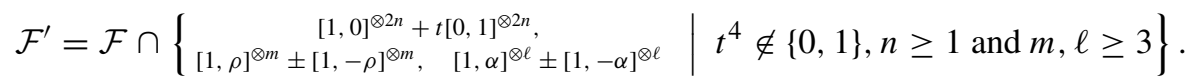

It follows that $\mathcal{F} \backslash \mathcal{F}^{\prime} \subseteq \bigcap_{k=3}^{5} S_{k}$.

Then $\mathcal{F}^{\prime} \nsubseteq S_{k}$ for all $3 \leq k \leq 5$. Indeed if $\mathcal{F}^{\prime} \subseteq S_{k}$ for some $3 \leq k \leq 5$, then $\mathcal{F} \subseteq S_{k}$, contrary to the given condition of the theorem. Let

$$
S=\mathcal{F}^{\prime} \cap\left\{[1, \rho]^{\otimes m} \pm[1,-\rho]^{\otimes m} \mid m \geq 3\right\} \quad \text { and } \quad T=\mathcal{F}^{\prime} \cap\left\{[1, \alpha]^{\otimes \ell} \pm[1,-\alpha]^{\otimes \ell} \mid \ell \geq 3\right\} .
$$

If $S \neq \emptyset$ and $T \neq \emptyset$, then there exist $g, h \in \mathcal{F}^{\prime}$ such that $g=[1, \alpha]^{\otimes m} \pm[1,-\alpha]^{\otimes m}$ and $h=[1, \rho]^{\otimes \ell} \pm[1,-\rho]^{\otimes \ell}$, where $m, \ell \geq 3$. By Lemma 11.6, we can get $[1,0]$ or $[0,1]$ from $f$. If we have $[1,0]$, then we have $g^{\prime}=\partial_{[1,0]}^{m-3}(g)=[1, \alpha]^{\otimes 3} \pm[1,-\alpha]^{\otimes 3}$ and $h^{\prime}=\partial_{[1,0]}^{\ell-3}(h)=[1, \rho]^{\otimes 3} \pm[1,-\rho]^{\otimes 3}$, and are done by Lemma 11.10. If we have $[0,1]$, then the proof follows from a transformation by $\left[\begin{array}{ll}0 & 1 \\ 1 & 0\end{array}\right]$.

If exactly one of $S$ and $T$ is not empty, then there exists some $[1,0]^{\otimes 2 n}+t[0,1]^{\otimes 2 n}$ with $t^{4} \notin\{0,1\}$ in $\mathcal{F}^{\prime}$, since otherwise $\mathcal{F}^{\prime}$ would be contained in either $\mathscr{A}$ or $\mathscr{A}^{\dagger}$. This contradicts $\mathcal{F}^{\prime} \nsubseteq S_{k}$ for $3 \leq k \leq 5$. By taking $\partial^{n-1}$, we have [1,0,t]. Moreover, we have $g=[1, \alpha]^{\otimes \ell} \pm[1,-\alpha]^{\otimes \ell}$ or $h=[1, \rho]^{\otimes m} \pm[1,-\rho]^{\otimes m}$ in $\mathcal{F}^{\prime}$, where $m, \ell \geq 3$. By a similar proof as in the previous case, first getting $[0,1]$ or $[1,0]$ by Lemma 11.6, we can have $g^{\prime}=[1, \alpha]^{\otimes 3} \pm[1,-\alpha]^{\otimes 3}$ or $h^{\prime}=[1, \rho]^{\otimes 3} \pm[1,-\rho]^{\otimes 3}$ in $\mathcal{F}^{\prime}$. Thus Pl-\#CSP${ }^{2}\left(\mathcal{F}^{\prime}\right)$ is \#P-hard by Lemma 11.9. So Pl-\#CSP$(\mathcal{F})$ is \#P-hard.

If $S=\emptyset$ and $T=\emptyset$, then $\mathcal{F}^{\prime} \subseteq\left\{[1,0]^{\otimes 2 n}+t[0,1]^{\otimes 2 n} \mid t^{4} \notin\{0,1\}\right\} \subseteq \mathscr{P}$. This contradicts that $\mathcal{F}^{\prime} \nsubseteq S_{k}$ for $3 \leq \bar{k} \leq 5$.

Now we can prove the dichotomy for $\mathrm{Pl}-\mathrm{CSSP}^{2}$ with a single symmetric signature of odd arity.

Theorem 11.12 If $f$ is a symmetric signature of odd arity, then either $\mathrm{Pl}-\# \mathrm{CSP}^{2}(f)$ is \#P-hard or $f \in \mathscr{P} \cup \mathscr{\mathscr { A }} \cup \widetilde{\mathscr{M}}$.

Proof Let $f$ have arity $2 n+1$. If $2 n+1=1$, then $f \in \mathscr{P}$. If $2 n+1=3$, then we are done by Lemma 11.2. In the following, assume that $2 n+1 \geq 5$. Let $f^{\prime}=\partial(f)$. If $f^{\prime} \notin \mathscr{P} \cup \widetilde{\mathscr{A}} \cup \widetilde{\mathscr{M}}$, then Pl-\#CSP${ }^{2}\left(f^{\prime}\right)$ is \#P-hard by induction. Thus Pl-\#CSP ${ }^{2}(f)$ is \#P-hard as well. Otherwise, $f^{\prime} \in \mathscr{P} \cup \widetilde{\mathscr{A}} \cup \widetilde{\mathscr{M}}$.

If $f^{\prime} \in \widetilde{\mathscr{M}} \backslash(\mathscr{P} \cup \mathscr{A})$, then we are done by Lemma 11.3. So we can assume that $f^{\prime} \in \mathscr{P} \cup \tilde{\mathscr{A}}$. Note that $f^{\prime}$ has odd arity, so if $f^{\prime}$ does not satisfy the parity constraints, then we have $[a, b]$ with $a b \neq 0$ by Lemma 11.5 and we are done by Lemma 10.2. Otherwise, either $f^{\prime}$ is identically zero or, as $f^{\prime}$ has odd arity and satisfies the parity constraints, by Lemma 11.4

$$
f^{\prime} \in\left\{[1,0]^{\otimes 2 n-1},[0,1]^{\otimes 2 n-1},[1, \alpha]^{\otimes 2 n-1} \pm[1,-\alpha]^{\otimes 2 n-1},[1, \rho]^{\otimes 2 n-1} \pm[1,-\rho]^{\otimes 2 n-1}\right\} .
$$


If $f^{\prime} \equiv 0$, then $f=x[1, i]^{\otimes 2 n+1}+y[1,-i]^{2 n+1}$ by Lemma 9.5. If $x=0$ or $y=0$ or $\left[x y \neq 0 \wedge x^{4}=y^{4}\right]$, then $f \in \mathscr{A}$. Otherwise, $x y \neq 0 \wedge x^{4} \neq y^{4}$.

- For $2 n+1 \equiv 1(\bmod 4)$, we have $\partial_{=}^{\frac{n}{2}}(f)=2^{\frac{n}{2}}\{x[1, i]+y[1,-i]\}=2^{\frac{n}{2}}[x+$ $y,(x-y) i]$. Note that $x+y \neq 0, x-y \neq 0$ by $x^{4} \neq y^{4}$. Then we are done by Lemma 10.2.

- For $2 n+1 \equiv 3(\bmod 4)$, we have $f^{\prime \prime}=\partial_{=4}^{\frac{n-1}{2}}(f)=2^{\frac{n-1}{2}}\left\{x[1, i]^{\otimes 3}+\right.$ $\left.y[1,-i]^{\otimes 3}\right\}$. Note that $x y \neq 0$ and $f$ is non-degenerate. And by its second-order recurrence, $f \in\langle 1,0,1\rangle$. it follows from Lemma 9.11 that $f^{\prime \prime} \notin \mathscr{P} \cup \widetilde{\mathscr{A}} \cup \widetilde{\mathscr{M}}$ since $x^{4} \neq y^{4}$. Thus $\mathrm{Pl}$-\#CSP${ }^{2}\left(f^{\prime \prime}\right)$ is \#P-hard by Lemma 11.2. So $\mathrm{Pl} \# \operatorname{CSP}^{2}(f)$ is \#P-hard.

If $f^{\prime} \in\left\{[1,0]^{\otimes 2 n-1},[0,1]^{\otimes 2 n-1},[1, \alpha]^{\otimes 2 n-1} \pm[1,-\alpha]^{\otimes 2 n-1},[1, \rho]^{\otimes 2 n-1} \pm\right.$ $\left.[1,-\rho]^{\otimes 2 n-1}\right\}$, then we have $[1,0]$ or $[0,1]$ by Lemma 11.6. So if $f$ does not satisfy the parity constraints, then we have $[a, b]$ with $a b \neq 0$ by Lemma 11.7 and we are done by Lemma 10.2. So we can assume that $f$ satisfies the parity constraints in the following.

- For $f^{\prime}=[1,0]^{\otimes 2 n-1}, f=x[1, i]^{\otimes 2 n+1}+y[1,-i]^{2 n+1}+[1,0]^{\otimes 2 n+1}$ by Lemma 9.5. If $x=y=0$, then $f \in \mathscr{P}$. Otherwise, $(x, y) \neq(0,0)$.

Let $a=x+y, b=(x-y) i$, then $(a, b) \neq(0,0)$. Note that $f=$ $[1+a, b,-a,-b, \ldots, \pm a, \pm b]$. Since $1+a$ and $-a$ cannot be both 0 , by the parity constraints, we have $b=0$. And thus $a \neq 0$. Moreover we have $\partial^{n-1}\left([1,0]^{2 n-1}\right)=[1,0]$ and $f^{\prime \prime \prime}=\partial_{[1,0]}^{2 n-3}(f)=[1+a, 0,-a, 0, a]$. We note that $2 n-3 \geq 1$ and so $\partial_{[1,0]}^{2 n-3}$ is defined. Note that $f^{\prime \prime \prime}$ is a redundant signature and its compressed signature matrix $\left[\begin{array}{llll}1+a & 0 & -a \\ 0 & -a & 0 \\ -a & 0 & a\end{array}\right]$ is nonsingular, so $\mathrm{Pl}-\# \mathrm{CSP}^{2}\left(f^{\prime \prime \prime}\right)$ is \#P-hard by Lemma 9.25. Thus $\mathrm{Pl}-\# \mathrm{CSP}^{2}(f)$ is \#P-hard.

- For $f^{\prime}=[0,1]^{\otimes 2 n-1}$, the proof follows from the previous case by a transformation using $\left[\begin{array}{ll}0 & 1 \\ 1 & 0\end{array}\right]$.

- For $f^{\prime}=[1, \alpha]^{\otimes 2 n-1} \pm[1,-\alpha]^{\otimes 2 n-1}, f=x[1, i]^{\otimes 2 n+1}+y[1,-i]^{2 n+1}+$ $\frac{1}{1+\alpha^{2}}\left\{[1, \alpha]^{\otimes 2 n+1} \pm[1,-\alpha]^{\otimes 2 n+1}\right\}$ by Lemma 9.5. If $x=y=0$, then $f \in \mathscr{A}^{\dagger}$. Otherwise, $(x, y) \neq(0,0)$. Firstly, we construct $\left[1,0, \alpha^{2}\right]$ by $f$. Note that we have $f^{(4)}=\partial^{n-1}(f)=\left(1+\alpha^{2}\right)^{n-2}\left\{[1, \alpha]^{\otimes 3} \pm[1,-\alpha]^{\otimes 3}\right\}$.

If $f^{(4)}=\left(1+\alpha^{2}\right)^{n-2}\left\{[1, \alpha]^{\otimes 3}+[1,-\alpha]^{\otimes 3}\right\}$ with a + sign, we have $\partial\left(f^{(4)}\right)=$ $2\left(1+\alpha^{2}\right)^{n-1}[1,0]$ and $\partial_{[1,0]}\left(f^{(4)}\right)=2\left(1+\alpha^{2}\right)^{n-2}\left[1,0, \alpha^{2}\right]$.

If $f^{(4)}=\left(1+\alpha^{2}\right)^{n-2}\left\{[1, \alpha]^{\otimes 3}-[1,-\alpha]^{\otimes 3}\right\}$ with a - sign, we have $\partial\left(f^{(4)}\right)=$ $2 \alpha\left(1+\alpha^{2}\right)^{n-1}[0,1]$ and $\partial_{[0,1]}\left(f^{(4)}\right)=2 \alpha\left(1+\alpha^{2}\right)^{n-2}\left[1,0, \alpha^{2}\right]$.

In either case, we have $\left[1,0, \alpha^{2}\right]$. Then we have $f^{(5)}=\partial_{\left[1,0, \alpha^{2}\right]}^{n-1}(f)=(1-$ $\left.\alpha^{2}\right)^{n-1}\left\{x[1, i]^{\otimes 3}+y[1,-i]^{3}\right\}$. If $x=0$ or $y=0$ or $\left[x y \neq 0 \wedge x^{4}=y^{4}\right]$, then $f^{(5)} \in \mathscr{A} \backslash \mathscr{A}^{\dagger}$. By the eigenvalues, $f^{\prime} \in\langle 1,0, \pm i\rangle$, hence $f^{\prime} \in \mathscr{A}^{\dagger} \backslash$ $(\mathscr{P} \cup \mathscr{A} \cup \widetilde{\mathscr{M}})$ in this case. So $\mathrm{Pl}-\# \operatorname{CSP}^{2}\left(f^{(5)}, f^{\prime}\right)$ is \#P-hard by Theorem 11.11. 
Otherwise, $x y \neq 0$ and $x^{4} \neq y^{4}$. Then $f^{(5)} \notin \mathscr{P} \cup \widetilde{\mathscr{A}} \cup \widetilde{\mathscr{M}}$. Thus $\operatorname{Pl}-\# \operatorname{CSP}^{2}\left(f^{(5)}\right)$ is \#P-hard by Lemma 11.2. So $\mathrm{Pl}-\# \mathrm{CSP}^{2}(f)$ is \#P-hard.

The final case is $f^{\prime}=[1, \rho]^{\otimes 2 n-1} \pm[1,-\rho]^{\otimes 2 n-1}$.

- $\quad$ For $f^{\prime}=[1,1]^{\otimes 2 n-1}+[1,-1]^{\otimes 2 n-1}$,

$$
f=x[1, i]^{\otimes 2 n+1}+y[1,-i]^{2 n+1}+\frac{1}{2}\left\{[1,1]^{\otimes 2 n+1}+[1,-1]^{\otimes 2 n+1}\right\} .
$$

If $x=y=0$, then $f \in \mathscr{A}$. In the following, assume that $(x, y) \neq(0,0)$. Let $a=x+y, b=(x-y) i$, then $(a, b) \neq(0,0)$. Moreover, $f=$ $[a, b,-a,-b, \ldots, \pm b]+[1,0,1,0, \ldots, 0]=[a+1, b,-a+1,-b, a+$ $1, \ldots, \pm b]$. Since $a+1$ and $-a+1$ cannot be both 0 , and $f$ satisfies the parity constraints, we have $b=0$. Thus $f=[a+1,0,-a+1,0, a+1, \ldots, \pm a+1,0]$. As $b=0$ we have $a \neq 0$. Note that we have $\partial^{n}(f)=2^{n}[1,0]$. Thus we have $f^{(6)}=\partial_{[1,0]}^{2 n-3}(f)=[a+1,0,-a+1,0, a+1]$. The compressed signature matrix of $f^{(6)}$ is $\left[\begin{array}{lll}a+1 & 0 & -a+1 \\ 0 & -a+1 & 0 \\ -a+1 & 0 & a+1\end{array}\right]$ with determinant $4 a(1-a)$. If $a \neq 1$, then by $a \neq 0$, this determinant is nonzero. Thus the compressed signature matrix of $f^{(6)}$ is nonsingular and $\mathrm{Pl}-\# \mathrm{CSP}^{2}\left(f^{(6)}\right)$ is \#P-hard by Lemma 9.25. So $\mathrm{Pl}-\# \mathrm{CSP}^{2}(f)$ is \#P-hard.

If $a=1$, then we have $f^{(7)}=\partial_{[1,0]}^{2 n-4}(f)=2[1,0,0,0,1,0]$ of arity 5 (note that $2 n-4 \geq 0$ ). Consider the gadget in Fig. 30. We assign $[1,0,0,0,1,0]$ to both vertices. The signature of this gadget is redundant, and its compressed signature matrix is $\left[\begin{array}{lll}1 & 0 & 0 \\ 0 & 1 & 0 \\ 0 & 0 & 3\end{array}\right]$. Since this matrix is nonsingular, we are done by Lemma 9.25.

- $\quad$ For $f^{\prime}=[1,1]^{\otimes 2 n-1}-[1,-1]^{\otimes 2 n-1}$,

$$
f=x[1, i]^{\otimes 2 n+1}+y[1,-i]^{2 n+1}+\frac{1}{2}\left\{[1,1]^{\otimes 2 n+1}-[1,-1]^{\otimes 2 n+1}\right\} .
$$

After the holographic transformation by $\left[\begin{array}{ll}0 & 1 \\ 1 & 0\end{array}\right]$, we have $\mathrm{Pl}-\# \mathrm{CSP}^{2}\left(f, f^{\prime}\right) \equiv$ $\operatorname{Pl}-\# \operatorname{CSP}^{2}\left(\widehat{f}, \widehat{f}^{\prime}\right)$, where $\widehat{f}^{\prime}=[1,1]^{\otimes 2 n-1}+[1,-1]^{\otimes 2 n-1}$, and $\widehat{f}=$

Fig. 30 Gadget used to obtain a signature whose signature matrix is redundant

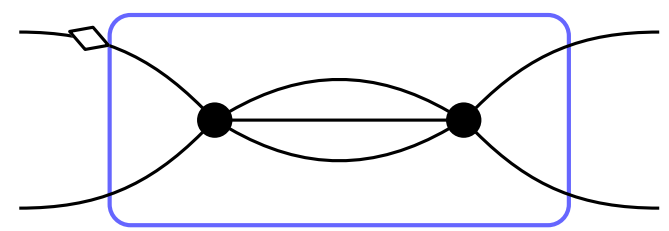


$x i^{2 n+1}[1,-i]^{\otimes 2 n+1}+y(-i)^{2 n+1}[1, i]^{2 n+1}+\frac{1}{2}\left\{[1,1]^{\otimes 2 n+1}+[1,-1]^{\otimes 2 n+1}\right\}$.

Thus we are done by the previous case.

- $\quad$ For $f^{\prime}=[1, i]^{\otimes 2 n-1}+[1,-i]^{\otimes 2 n-1}, f$ has arity $2 n+1$ and using Proposition 9.7 (the Explicit List for $\left.\int\left(f^{\prime}\right)\right), \int\left([1, \epsilon i]^{\otimes 2 n-1}\right)$ is a sum of $\lambda[1, \epsilon i]^{\otimes 2 n+1}$ with a signature having the $k$-th term of the form $-\frac{1}{2} k(\epsilon i)^{k}$ for $0 \leq k \leq 2 n+1$. Thus, we can write $f_{k}=\left(x-\frac{1}{2} k\right) i^{k}+\left(y-\frac{1}{2} k\right)(-i)^{k}$ for some $x$ and $y$, and for $0 \leq k \leq 2 n+1$ by Lemma 9.5 .

We have $\partial_{f^{\prime}}(=2 n)=\partial_{\partial(f)}(=2 n)=2[1,0]$ on LHS.

Let $a=x+y, b=(x-y) i$, then $f=[a, b,-a+2,-b, a-4, \ldots, \pm b]$. Since $a$ and $-a+2$ cannot be both 0 , and $f$ satisfies the parity constraints, we have $b=0$. Then we have $f^{(8)}=\partial_{[1,0]}^{2 n-3}(f)=[a, 0,-a+2,0, a-4]$. If $a \neq 2$, then the compressed signature matrix of $f^{(8)}$ is $\left[\begin{array}{lll}a & 0 & -a+2 \\ 0 & -a+2 & 0 \\ -a+2 & 0 & a-4\end{array}\right]$, and is nonsingular and we are done by Lemma $9.2 \overline{5}$.

For $a=2$, we have $\partial_{[1,0]}^{2 n-4}(f)=2[1,0,0,0,-1,0]$. Consider the gadget in Fig. 30. We assign $[1,0,0,0,-1,0]$ to both vertices. The signature of this gadget is redundant, and its compressed signature matrix is $\left[\begin{array}{lll}1 & 0 & 0 \\ 0 & 1 & 0 \\ 0 & 0 & 3\end{array}\right]$. Since this matrix is nonsingular, we are done by Lemma 9.25.

- For $f^{\prime}=[1, i]^{\otimes 2 n-1}-[1,-i]^{\otimes 2 n-1}$, the proof follows from the previous case by a holographic transformation using $\left[\begin{array}{ll}0 & 1 \\ 1 & 0\end{array}\right]$.

By Theorem 11.11 and Theorem 11.12, we have the following dichotomy theorem.

Theorem 11.13 For any set of symmetric signatures $\mathcal{F}$ that contains at least one nonzero signature with odd arity, if $\mathcal{F} \subseteq \mathscr{P}$, or $\mathscr{A}$, or $\mathscr{A}^{\dagger}$, or $\widehat{\mathscr{M}}$, or $\widehat{\mathscr{M}}^{\dagger}$, then $P l$-\#CSP $P^{2}(\mathcal{F})$ is tractable. Otherwise, $P l-\# C S P^{2}(\mathcal{F})$ is \#P-hard.

\section{Arity 4 Dichotomy for PI-\#CSP ${ }^{2}(f)$}

The goal of this section is a dichotomy theorem for $\mathrm{Pl}^{-\# \mathrm{CSP}^{2}}(f)$ when $f$ is a symmetric signature of arity 4 . Frequently our first test uses the determinantal criterion of a redundant signature of arity 4 based on Lemma 9.25.

Lemma 12.1 Let $f$ be an arity 4 symmetric signature. If the signature matrix of $f$ is redundant, and its compressed form is nonsingular, then $\mathrm{Pl}-\# \mathrm{CSP}^{2}(f)$ is \#P-hard.

Proof Since Pl-Holant $(f) \leq_{T} \mathrm{Pl}_{\mathrm{ACSP}}(f)$, we are done by Lemma 9.25. 
Next we introduce a trick which we call the "Three Stooges". For $f=$ $[a, b, c, d, e]$, define

$$
\begin{aligned}
f^{\times} & =[a, c, e] \\
f^{\times \times} & =\left[a^{2}+c^{2}+2 b^{2}, a c+c e+2 b d, c^{2}+e^{2}+2 d^{2}\right], \quad \text { and } \\
f^{\times} & =\left[a^{2}+c^{2}+2 b^{2}, b^{2}+d^{2}+2 c^{2}, c^{2}+e^{2}+2 d^{2}\right] .
\end{aligned}
$$

The following lemma is proved by the technique of domain pairing.

Lemma 12.2 If $f=\left[a, b, c, d\right.$, e], then $\mathrm{Pl}-\# \operatorname{CSP}\left(f^{\times}, f^{\ngtr}, f^{\times \times}\right) \leq_{T} \operatorname{Pl}^{\times} \# \mathrm{CSP}^{2}(f)$.

Proof Let $f^{\prime}$ be the signature of the gadget in Fig. 31 and $f^{\prime \prime}$ be the signature of the gadget in Fig. 31 rotated $90^{\circ}$. Then $f^{\prime}$ and $f^{\prime \prime}$ have the first and the second signature matrix below respectively:

$$
\begin{aligned}
& {\left[\begin{array}{llll}
a^{2}+c^{2}+2 b^{2} & a b+c d+2 b c & a b+c d+2 b c & a c+c e+2 b d \\
a b+c d+2 b c & b^{2}+d^{2}+2 c^{2} & b^{2}+d^{2}+2 c^{2} & b c+d e+2 c d \\
a b+c d+2 b c & b^{2}+d^{2}+2 c^{2} & b^{2}+d^{2}+2 c^{2} & b c+d e+2 c d \\
a c+c e+2 b d & b c+d e+2 c d & b c+d e+2 c d & c^{2}+e^{2}+2 d^{2}
\end{array}\right]} \\
& {\left[\begin{array}{llll}
a^{2}+c^{2}+2 b^{2} & a b+c d+2 b c & a b+c d+2 b c & b^{2}+d^{2}+2 c^{2} \\
a b+c d+2 b c & a c+c e+2 b d & b^{2}+d^{2}+2 c^{2} & b c+d e+2 c d \\
a b+c d+2 b c & b^{2}+d^{2}+2 c^{2} & a c+c e+2 b d & b c+d e+2 c d \\
b^{2}+d^{2}+2 c^{2} & b c+d e+2 c d & b c+d e+2 c d & c^{2}+e^{2}+2 d^{2}
\end{array}\right]}
\end{aligned}
$$

Fig. 31 Gadget used in Lemma 12.2. Both vertices are assigned $f$

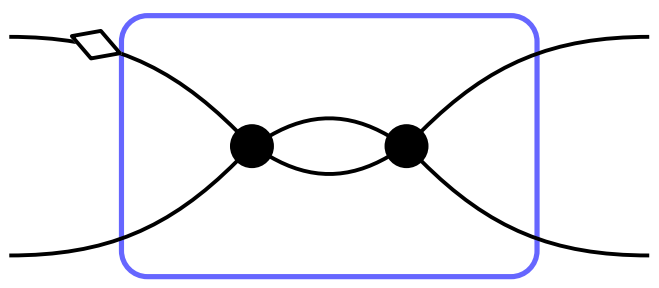


We highlight the relevant entries in the display below (in fact, readers should only focus on the entries highlighted; see Fig. 2 in Part I for an illustration of the rotation operation):

$$
\begin{gathered}
{\left[\begin{array}{cccc}
a^{2}+c^{2}+2 b^{2} & * & * & a c+c e+2 b d \\
* & b^{2}+d^{2}+2 c^{2} & * & * \\
* & * & b^{2}+d^{2}+2 c^{2} & * \\
a c+c e+2 b d & * & * & c^{2}+e^{2}+2 d^{2}
\end{array}\right]} \\
{\left[\begin{array}{cccc}
a^{2}+c^{2}+2 b^{2} & * & * & b^{2}+d^{2}+2 c^{2} \\
* & a c+c e+2 b d & * & \\
* & * & a c+c e+2 b d & * \\
b^{2}+d^{2}+2 c^{2} & * & * & c^{2}+e^{2}+2 d^{2}
\end{array}\right]}
\end{gathered}
$$

For any instance of $\mathrm{Pl}$-\#CSP$\left(f^{\times}, f^{\times}, f^{\times \times}\right)$, we replace each edge $e$ by two edges that connect the same incident nodes of $e$. For each variable node that is connected to $k$ edges, we replace its label $=k$ by $=2 k$. We replace each occurrence of $f^{\times}, f^{\times}, f^{\times \times}$by $f, f^{\prime}, f^{\prime \prime}$ as a constraint respectively. Then the new instance is a problem in $\mathrm{Pl}-\# \operatorname{CSP}^{2}\left(f, f^{\prime}, f^{\prime \prime}\right)$ and has the same value as the given instance of

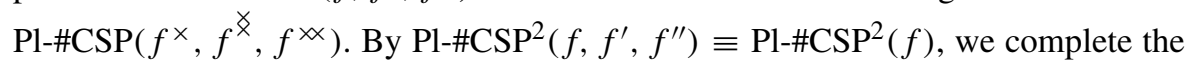
proof.

We demonstrate a simple use of the "Three Stooges" in the following lemma.

Lemma 12.3 If $a^{4} \notin\{0,1\}$, then $\mathrm{Pl}-\# \mathrm{CSP}^{2}\left(\left[1,0, a, 0, a^{2}\right]\right)$ is \#P-hard.

Proof For $f=\left[1,0, a, 0, a^{2}\right]$, we have $f^{\times}=\left[1, a, a^{2}\right]$ and $f^{\times}=[1+$ $\left.a^{2}, 2 a^{2}, a^{2}\left(1+a^{2}\right)\right]$. By Lemma 9.8, $f^{\times} \notin \mathscr{A} \cup \widehat{\mathscr{M}}$ since $a^{4} \notin\{0,1\}$. By the same reason and Lemma 9.8, the only possibility for $f^{\ngtr} \in \mathscr{P}$ is being degenerate. Thus $a^{2}\left(1+a^{2}\right)^{2}=4 a^{4}$. This implies that $a=0$ or $a= \pm 1$; a contradiction. This implies that $f^{\times}$and $f^{\ngtr}$ cannot both be in $\mathscr{P}, \mathscr{A}$, or $\widehat{\mathscr{M}}$. Thus $\mathrm{Pl} \# \operatorname{CSP}\left(f^{\times}, f^{\ngtr}\right)$ is \#P-hard by Theorem 9.22. Then by Lemma 12.2, $\mathrm{Pl}-\# \mathrm{CSP}^{2}(f)$ is \#P-hard.

Lemma 12.4 Let $f=[1,1]^{\otimes 4}+a[1,-1]^{\otimes 4}$, where $a^{4} \neq 0,1$. Then $\mathrm{Pl}^{-\# C_{S P}^{2}}(f)$ is \#P-hard.

Proof Under a holographic transformation by $H=\left[\begin{array}{ll}1 & 1 \\ 1 & -1\end{array}\right]$, we have

$$
\begin{aligned}
\operatorname{Pl-\# CSP} & (f) \\
& \equiv \operatorname{Pl}-H o l a n t\left(\mathcal{E} \mathcal{Q}_{2} \mid f\right) \\
& \equiv \operatorname{Pl-Holant}([1,0,1],[1,0,1,0,1], \ldots \mid \hat{f}),
\end{aligned}
$$


where $\hat{f}=\left(H^{-1}\right)^{\otimes 4} f=[1,0,0,0, a]$. By Lemma 12.3, $\operatorname{Pl}_{-\# C S P}{ }^{2}\left(\left[1,0, a, 0, a^{2}\right]\right)$ is \#P-hard, and we have

$$
\begin{aligned}
\mathrm{Pl}-\# \operatorname{CSP}^{2}\left(\left[1,0, a, 0, a^{2}\right]\right) & \equiv \operatorname{Pl}-\operatorname{Holant}\left(\mathcal{E}_{\left.\mathcal{Q}_{2} \mid\left[1,0, a, 0, a^{2}\right]\right)}\right. \\
& \equiv \operatorname{Pl-Holant}\left([1,0, a],\left[1,0,0,0, a^{2}\right], \cdots \mid[1,0,1,0,1]\right) \\
& \leq \operatorname{Pl}-\operatorname{Holant}\left([1,0, a],\left[1,0,0,0, a^{2}\right], \cdots \mid[1,0,1],[1,0,1,0,1], \cdots\right),
\end{aligned}
$$

where the second equivalence $\equiv$ is by a holographic transformation with $\left[\begin{array}{cc}1 & 0 \\ 0 & \sqrt{a}\end{array}\right]$.

The problem in (12.15) can simulate the problem in (12.18). With $[1,0,1]$ on the left and $\hat{f}$ on the right in $(12.15)$, we can get $\partial(\hat{f})=[1,0, a]$ on the right. Now consider the gadget in Fig. 32 . We assign $\hat{f}$ to the circle vertices, $={ }_{2}$ to the square vertices, and $[1,0, a]$ to the triangle vertex. If there are $k-1$ occurrences of the dashed subgadget, then the signature of this gadget is $\left[1,0, \ldots, 0, a^{k}\right]$ of arity $2 k$. Thus

$$
\leq \quad \begin{aligned}
& \text { Pl-Holant }\left([1,0,1],[1,0,1,0,1], \cdots \mid[1,0, a],\left[1,0,0,0, a^{2}\right], \cdots\right) \\
& \operatorname{Pl-Holant}([1,0,1],[1,0,1,0,1], \cdots \mid \hat{f}) .
\end{aligned}
$$

Then combining three reductions, we have $\operatorname{Pl}-\# \mathrm{CSP}^{2}\left(\left[1,0, a, 0, a^{2}\right]\right) \leq$ $\mathrm{Pl}$ \#CSP${ }^{2}(f)$, where $a^{4} \neq 0,1$. Thus $\mathrm{Pl}$ \#CSP${ }^{2}(f)$ is \#P-hard by Lemma 12.3.

Now we are ready to prove the following theorem.

Theorem 12.5 Let $f$ be a symmetric signature of arity 4 , then $\mathrm{Pl}-\# \mathrm{CSP}^{2}(f)$ is \#Phard or $f \in \mathscr{P} \cup \mathscr{A} \cup \widetilde{\mathscr{M}}$.

Proof The first step is to apply Lemma 12.2 to $f^{\times}$. For $f=\left[f_{0}, f_{1}, f_{2}, f_{3}, f_{4}\right]$ we have $f^{\times}=\left[f_{0}, f_{2}, f_{4}\right]$. If $\mathrm{Pl}$-\#CSP $\left(f^{\times}\right)$is \#P-hard, then $\mathrm{Pl}$-\#CSP${ }^{2}(f)$ is \#P-hard by Lemma 12.2. In the following, assume that $\operatorname{Pl} \# \operatorname{CSP}\left(f^{\times}\right)$is tractable. So we can use the dichotomy Theorem 9.21 for $d=1$, i.e., for $\left[f_{0}, f_{2}, f_{4}\right]$, one of the following conditions holds:

1. $f_{0} f_{4}=f_{2}^{2}$;

2. $f_{0}=f_{4}=0$

3. $f_{2}=0$;

4. $f_{0} f_{4}=-f_{2}^{2}$ and $f_{0}=-f_{4} \neq 0$;

5. $f_{0}=f_{4} \neq 0$.

Now we can characterize the form of $\left[f_{0}, f_{2}, f_{4}\right]$.

- If $f_{2}=0$, then $\left[f_{0}, f_{2}, f_{4}\right]$ takes the form $[0,0,0],[0,0,1],[1,0,0]$ or $[1,0, a]$ with $a \neq 0$ up to a scalar;

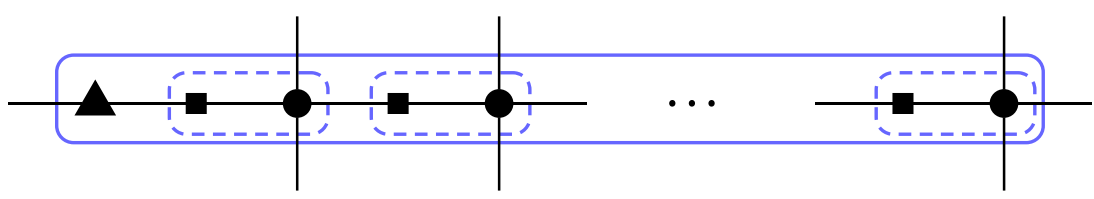

Fig. 32 Gadget $\Gamma_{k}$, which has $k-1$ copies of the dashed box. Circle vertices are assigned $\hat{f}$, square vertices are assigned $=_{2}$, and the triangle vertex is assigned $[1,0, a]$ 
- if $f_{2} \neq 0, f_{0}=0$ or $f_{4}=0$, then $\left[f_{0}, f_{2}, f_{4}\right]$ takes the form $[0,1,0]$ up to a scalar;

- if all of $f_{0}, f_{2}, f_{4}$ are nonzero, then up to a scalar, $\left[f_{0}, f_{2}, f_{4}\right]$ has to take the form $\left[1, r, r^{2}\right],[1,1,-1],[1,-1,-1]$ or $[1, b, 1]$ with $r \neq 0, b \neq 0$;

i.e., $\left[f_{0}, f_{2}, f_{4}\right]$ takes one of the following form

$[0,0,0],[1,0,0],[0,0,1],\left[1, r, r^{2}\right],[0,1,0],[1,0, a],[1,1,-1],[1,-1,-1]$, or $[1, b, 1]$

up to a scalar, where $r \neq 0, a \neq 0$, and $b^{2} \notin\{0,1\}$.

Case 1: $\left[f_{0}, f_{2}, f_{4}\right]=[0,0,0]$

In this case, $f=[0, x, 0, y, 0]$ and $f^{\ngtr}=\left[2 x^{2}, x^{2}+y^{2}, 2 y^{2}\right]$.

- If $x^{2}=y^{2}$, then $f=[0, x, 0, \pm x, 0] \in \mathscr{A}$.

- If $x^{2}=-y^{2}$, then $f=[0,1,0, \pm i, 0] \in \mathscr{A}^{\dagger}$ since $\left[\begin{array}{ll}1 & 0 \\ 0 & \sqrt{i}\end{array}\right]^{\otimes 4} f \in \mathscr{A}$.

- If $x^{4} \neq y^{4}$, then $\mathrm{Pl}$-\#CSP$\left(f^{\searrow}\right)$ is \#P-hard by Theorem 9.21, so $\mathrm{Pl}$-\#CSP${ }^{2}(f)$ is \#P-hard by Lemma 12.2.

Case 2: $\left[f_{0}, f_{2}, f_{4}\right]=[1,0,0]$ or $[0,0,1]$

We prove the case for $\left[f_{0}, f_{2}, f_{4}\right]=[1,0,0]$, i.e., $f=[1, x, 0, y, 0]$. The other case is similar.

Note that we have $\partial(f)=[1, x+y, 0]$. If $x+y \neq 0$, then $\operatorname{Pl}_{-1 C S P}([1, x+y, 0])$ is \#P-hard by Theorem 9.21. Thus $\mathrm{Pl}-\# \mathrm{CSP}^{2}(f)$ is \#P-hard.

If $x=-y \neq 0$, then $\mathrm{Pl}$-\#CSP$(f)$ is \#P-hard by Lemma 12.1.

If $x=-y=0$, then $f=[1,0]^{\otimes 4} \in \mathscr{P}$.

Case 3: $\left[f_{0}, f_{2}, f_{4}\right]=\left[1, r, r^{2}\right]$ with $r \neq 0$

In this case, $f=\left[1, x, r, y, r^{2}\right]$. If $r x \neq y$, then $\mathrm{Pl}-\# \mathrm{CSP}^{2}(f)$ is \#P-hard by Lemma 12.1. Otherwise, $f=\left[1, x, r, x r, r^{2}\right]$. Then we have $\partial(f)=(1+$ $r)[1, x, r]$. If $r \neq-1$, then we have [1, $x, r]$. In the following we will separate out the cases according to value of $r$.

For $r^{4} \neq 1$ in $f=\left[1, x, r, x r, r^{2}\right]$.

- If $x=0$, then $f=\left[1,0, r, 0, r^{2}\right]$, and $\mathrm{Pl}-\# \mathrm{CSP}^{2}(f)$ is \#P-hard by Lemma 12.3.

- If $x^{2}=r$, then $f=[1, x]^{\otimes 4} \in \mathscr{P}$.

- If $x^{2} \neq r$ and $x \neq 0$, then Pl-\#CSP ${ }^{2}([1, x, r])$ is \#P-hard by Theorem 9.21. Thus Pl-\#CSP${ }^{2}(f)$ is \#P-hard.

For $r=1$, then $f=[1, x, 1, x, 1]$.

- If $x^{4}=0$ or 1 , then $f \in \mathscr{A}$. 
- If $x^{4} \neq 0,1$, then let $a=\frac{1-x}{1+x}$ and we have $a^{4} \neq 0,1$ by Lemma 9.3. Note that $f=\frac{1}{1+a}\left\{[1,1]^{\otimes 4}+a[1,-1]^{\otimes 4}\right\}$. By Lemma $12.4, \mathrm{Pl}$ \#CSP${ }^{2}(f)$ is \#P-hard. For $r=-1$, then $f=[1, x,-1,-x, 1]$.

- If $x^{4}=0$ or 1 , then $f \in \mathscr{A}$.

- If $x^{4} \neq 0,1$, then let $a=\frac{1+x i}{1-x i}$ and we have $a^{4} \neq 0,1$ by Lemma 9.3. Note that $f=\frac{1}{a+1}\left\{[1, i]^{\otimes 4}+a[1,-i]^{\otimes 4}\right\}$. Thus we have $[1,0,-1]^{\otimes 2}$ on the left by Lemma 9.20. Under the holographic transformation by $\left[\begin{array}{ll}1 & 0 \\ 0 & i\end{array}\right]$, this $[1,0,-1]^{\otimes 2}$ is transformed to $[1,0,1]^{\otimes 2}$, and we have $\mathrm{Pl} \# \mathrm{CSP}^{2}(f) \equiv_{T} \operatorname{Pl}-\operatorname{Holant}\left(\mathcal{E} \mathcal{Q}_{4} \cup\left\{[1,0,1]^{\otimes 2},[1,0,-1],[1,0,0,0,0,0,-1], \cdots\right\} \mid f^{\prime}\right)$,

where $f^{\prime}=\frac{1}{1+a}\left\{[1,1]^{\otimes 4}+a[1,-1]^{\otimes 4}\right\}$. Now having $[1,0,1]^{\otimes 2}$ on the left, we can form a pair of self loops in a planar way for a pair of adjacent $f^{\prime}$ and get $\left(\partial\left(f^{\prime}\right)\right)^{\otimes 2}=\left(\frac{2}{1+a}[1+a, 1-a, 1+a]\right)^{\otimes 2}$ on the right side. Since we have $[1,0,1]^{\otimes 2}$ on the left side, we can obtain $[1,0,1]^{\otimes 2}$ on the right side by interpolation using $[1+a, 1-a, 1+a]^{\otimes 2}$. Note that the matrix $\left[\begin{array}{cc}1+a & 1-a \\ 1-a & 1+a\end{array}\right]$ can be diagonalized by $\left[\begin{array}{ll}1 & 1 \\ 1 & -1\end{array}\right]$. This implies that

$$
\mathrm{Pl}-\# \mathrm{CSP}^{4}\left(f^{\prime},[1,0,1]^{\otimes 2}\right) \leq_{T}
$$$$
\operatorname{Pl} \text {-Holant }\left(\mathcal{E} \mathcal{Q}_{4} \cup\left\{[1,0,1]^{\otimes 2},[1,0,-1],[1,0,0,0,0,0,-1], \cdots\right\} \mid f^{\prime}\right) \text {. }
$$

Then by (12.19) and Lemma 10.4, we have

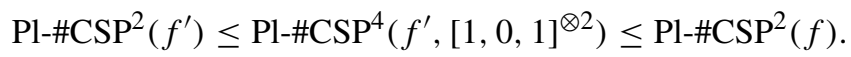

By Lemma 12.4, $\mathrm{Pl}$-\#CSP${ }^{2}\left(f^{\prime}\right)$ is \#P-hard. Thus $\mathrm{Pl}$ \#CSP${ }^{2}(f)$ is \#P-hard.

For $r^{2}=-1$, then $r= \pm i$ in $f=[1, x, r, x r,-1]$.

- If $x=0$, then $f=[1,0, r, 0,-1] \in \mathscr{A}^{\dagger}$ since $\left[\begin{array}{ll}1 & 0 \\ 0 & \sqrt{i}\end{array}\right]^{\otimes 4} f=$ $[1,0, \pm 1,0,1] \in \mathscr{A}$.

- If $x^{2}=r$, then $f=[1, x]^{\otimes 4} \in \mathscr{P}$.

- If $x^{2}=-r$, then $f=\left[1, x,-x^{2},-x^{3},-1\right] \in \mathscr{A}^{\dagger}$ since $\left[\begin{array}{ll}1 & 0 \\ 0 & x^{-1}\end{array}\right]^{\otimes 4} f \in \mathscr{A}$, with $x^{4}=-1$.

- If $x^{4} \neq 0,-1$, thus $x^{2} \neq \pm r$. Then $\mathrm{Pl}-\# \mathrm{CSP}^{2}([1, x, r])$ is \#P-hard by Theorem 9.21. Thus $\mathrm{Pl}$-\#CSP${ }^{2}(f)$ is \#P-hard.

Case 4: $\left[f_{0}, f_{2}, f_{4}\right]=[0,1,0]$ 
In this case, $f=[0, x, 1, y, 0]$. We first apply Lemma 12.1 and calculate the determinant of the compressed matrix for $f$, which is $2 x y-1$. If $x y \neq \frac{1}{2}$, then $\mathrm{Pl}-\# \mathrm{CSP}^{2}(f)$ is \#P-hard by Lemma 12.1.

If $x y=\frac{1}{2}$ and $x-y=0$, then $f=\left[0, \frac{1}{\sqrt{2}}, 1, \frac{1}{\sqrt{2}}, 0\right]$ or $f=\left[0,-\frac{1}{\sqrt{2}}, 1,-\frac{1}{\sqrt{2}}, 0\right]$. Both are in $\widehat{\mathscr{M}}$, by Lemma 9.18.

If $x y=\frac{1}{2}$ and $x+y=0$, then $f=\left[0, \frac{i}{\sqrt{2}}, 1,-\frac{i}{\sqrt{2}}, 0\right]$ or $f=$ $\left[0,-\frac{i}{\sqrt{2}}, 1, \frac{i}{\sqrt{2}}, 0\right]$. Both are in $\widehat{\mathscr{M}^{\dagger}}$, by Lemma 9.18. In fact from the previous line with $\left[0, \pm \frac{1}{\sqrt{2}}, 1, \pm \frac{1}{\sqrt{2}}, 0\right]$, we can see directly $\left[\begin{array}{ll}1 & 0 \\ 0 & i\end{array}\right]^{\otimes 4} f \in \widehat{\mathscr{M}}$.

In the following we have $x y=\frac{1}{2}$ and $x^{2} \neq y^{2}$. Then $f^{\vee}=\left[1+2 x^{2}, 2+x^{2}+\right.$ $\left.y^{2}, 1+2 y^{2}\right]$ and $f^{\infty \times}=\left[1+2 x^{2}, 1,1+2 y^{2}\right]$. We will prove that $\operatorname{Pl}$-\#CSP$\left(f^{\ngtr}, f^{\infty \times}\right)$ is \#P-hard by showing that $f^{\ngtr}, f^{\times x}$ cannot be both in the same $\mathscr{P}, \mathscr{A}$, or $\widehat{\mathscr{M}}$.

- $\quad$ By $x^{2} \neq y^{2}$ and Lemma 9.8, we have $f^{\infty \times} \notin \widehat{\mathscr{M}}$.

- Suppose $\left\{f^{\ngtr}, f^{\times}\right\} \subseteq \mathscr{P} . f^{x}$ is not of the form $[1,0, a]$, and also not of the form $[0,1,0]$ since $1+2 x^{2} \neq 1+2 y^{2}$. Thus $f^{\infty \times}$ is degenerate, i.e., $\left(1+2 x^{2}\right)\left(1+2 y^{2}\right)=1$. Note that $f^{x}$ is not of the form $[0,1,0]$ since $1+2 x^{2} \neq$ $1+2 y^{2}$. If $f^{\searrow}$ is of the form $[1,0, a]$, then $x^{2}+y^{2}=-2$. Then together with $x y=\frac{1}{2}$ we obtain $\left(1+2 x^{2}\right)\left(1+2 y^{2}\right)=-2 \neq 1$. This contradicts that $f^{\times x}$ is degenerate. Thus $f^{\vee}$ and $f^{x \times}$ are both degenerate. Then we have

$$
\begin{gathered}
\left(1+2 x^{2}\right)\left(1+2 y^{2}\right)=\left(x^{2}+y^{2}+2\right)^{2}, \\
\left(1+2 x^{2}\right)\left(1+2 y^{2}\right)=1 .
\end{gathered}
$$

We show that these two equations contradict the known relation $x y=1 / 2$. Note that the left sides of (12.21) and (12.22) are the same. Thus we have $\left(x^{2}+y^{2}+2\right)^{2}=1$, i.e., $x^{2}+y^{2}=-3$ or $x^{2}+y^{2}=-1$. By expanding (12.22), we have $2\left(x^{2}+y^{2}\right)+4 x^{2} y^{2}=0$. If $x^{2}+y^{2}=-3$ or -1 , then $4 x^{2} y^{2}=6$ or 2 respectively. Both possibilities contradict that $x y=\frac{1}{2}$. Thus $f^{\times}$and $f^{\times x}$ cannot both belong to $\mathscr{P}$.

- Suppose $\left\{f^{\times}, f^{x \times}\right\} \subseteq \mathscr{A}$. By $f^{\times x} \in \mathscr{A}$ and the middle term is nonzero, by Corollary 9.9 we have $1+2 x^{2}= \pm\left(1+2 y^{2}\right)$. Since $x^{2} \neq y^{2}$, we have $1+2 x^{2}=-1-2 y^{2}$. This leads to $(x+y)^{2}=0$ by using $x y=\frac{1}{2}$. This contradicts $x^{2} \neq y^{2}$.

We have proved that $f^{\times}, f^{\times x}$ cannot be both in $\mathscr{P}$, or $\mathscr{A}$, or $\widehat{\mathscr{M}}$. Thus $\mathrm{Pl}$-\#CSP $\left(f^{\times}, f^{\infty \times}\right)$ is \#P-hard by Theorem 9.22. So Pl-\#CSP${ }^{2}(f)$ is \#P-hard by Lemma 12.2. 
Case 5: $\left[f_{0}, f_{2}, f_{4}\right]=[1,0, a]$ with $a \neq 0$

In this case, $f=[1, x, 0, y, a]$. We first apply Lemma 12.1 and calculate the determinant of the compressed matrix for $f$, which is $-\left(a x^{2}+y^{2}\right)$. If $a x^{2}+$ $y^{2} \neq 0$, then $\mathrm{Pl}$ \#CSP${ }^{2}(f)$ is \#P-hard by Lemma 12.1. In the following we assume $a x^{2}+y^{2}=0$.

If $x=y=0$, then $f \in \mathscr{P}$.

If $x=y \neq 0$, then $a=-1$. So $f=[1, x, 0, x,-1] \in \widehat{\mathscr{M}^{\dagger}}$, by Corollary 9.18.

If $x=-y \neq 0$, then $a=-1$. So $f=[1, x, 0,-x,-1] \in \widehat{\mathscr{M}}$, by Corollary 9.18 . Now we assume $a x^{2}+y^{2}=0$ and $x^{2} \neq y^{2}$. Then $a \neq-1$ and $x y \neq 0$ by $a \neq 0$. In this case, the "Three Stooges" are

$f^{\times}=[1,0, a], \quad f^{\ngtr}=\left[1+2 x^{2}, x^{2}+y^{2}, a^{2}+2 y^{2}\right], \quad$ and $\quad f^{\infty \times}=\left[1+2 x^{2}, 2 x y, a^{2}+2 y^{2}\right]$.

By $a x^{2}+y^{2}=0$, we have

$$
f^{\ngtr}=\left[1+2 x^{2},(1-a) x^{2}, a^{2}-2 a x^{2}\right] \quad \text { and } \quad f^{\not x}=\left[1+2 x^{2}, 2 x y, a^{2}-2 a x^{2}\right] .
$$

We will prove that $\mathrm{Pl}$-\#CSP $\left(f^{\times}, f^{\times}, f^{\times \times}\right)$is \#P-hard by showing that $f^{\times}, f^{\times}$and $f^{\times \times}$cannot be all in the same $\mathscr{P}, \mathscr{A}$, or $\widehat{\mathscr{M}}$.

- $\quad$ Suppose $\left\{f^{\times}, f^{\times}\right\} \subseteq \widehat{\mathscr{M}}$. Note that $a \neq-1$. If $f^{\times} \in \widehat{\mathscr{M}}$, we have $a=1$ by Lemma 9.8. Then by $f^{x} \in \widehat{\mathscr{M}}$ and Lemma 9.8, we have $1+2 x^{2}=1-2 x^{2}$ or $2 x y=0$. This contradicts that $x y=\frac{1}{2}$.

- Suppose $\left\{f^{\times}, f^{\times}\right\} \subseteq \mathscr{P}$. If $1+2 x^{2}$ and $a^{2}-2 a x^{2}$ are both zero, then $a=0$ or -1 . This contradicts that $a \neq 0$ and $a \neq-1$. Thus $f^{\times}, f^{\times \times}$are not of the form $[0,1,0]$. By $x y \neq 0, f^{x}$ is not of the form $[1,0, c]$ with $c \neq 0$. Thus $f^{x \times}$ is degenerate by Lemma 9.8 , i.e.,

$$
\left(1+2 x^{2}\right)\left(a^{2}-2 a x^{2}\right)=4 x^{2} y^{2}=-4 a x^{4},
$$

where the last equality is by $a x^{2}+y^{2}=0$.

If $a=1$, we have $1-4 x^{4}=-4 x^{4}$ by (12.23). This is a contradiction.

If $a \neq 1$, then $f^{\ngtr}$ is not of the form $[1,0, c]$ with $c \neq 0$. Thus $f^{\ngtr}$ is degenerate by $f^{\gtrless} \in \mathscr{P}$, i.e.,

$$
\left(1+2 x^{2}\right)\left(a^{2}-2 a x^{2}\right)=(1-a)^{2} x^{4} .
$$

Then by (12.23), we have $-4 a x^{4}=(1-a)^{2} x^{4}$. This implies that $-4 a=$ $(1-a)^{2}$ by $x \neq 0$. Then $(1+a)^{2}=0$, contradicting $a \neq \pm 1$.

- Suppose $\left\{f^{\times}, f^{\times}\right\} \subseteq \mathscr{A}$. By $f^{\times} \in \mathscr{A}$, and $a \neq 0$, we get $a^{4}=1$ from Lemma 9.8. It follows that $a=1$ or $a^{2}=-1$, as we have $a \neq-1$.

For $a=1$, the equation $a x^{2}+y^{2}=0$ gives us $y^{2}=-x^{2}$. Then from Corollary 9.9 we have

$$
\left(1+2 x^{2}\right)^{2}=\left(1-2 x^{2}\right)^{2}
$$

by $f^{x \times} \in \mathscr{A}$ and $2 x y \neq 0$. Thus $x=0$. This is a contradiction.

For $a^{2}=-1$, by $f^{\infty x} \in \mathscr{A}$ and $2 x y \neq 0$, we have $\left(1+2 x^{2}\right)^{2}=(-1-$ $\left.2 a x^{2}\right)^{2}$ by Corollary 9.9. $1+2 x^{2}=1+2 a x^{2}$ leads to a contradiction $a=1$, 
hence $1+2 x^{2}=-\left(1+2 a x^{2}\right)$. Then $x^{2}=-\frac{1}{a+1}$ and $f^{x \times}=\left[\frac{a-1}{a+1}, 2 x y, \frac{a-1}{a+1}\right]$. Note that $a+1 \neq 0$. We observe that the norm of $x^{2}$ is $\frac{1}{\sqrt{2}}$ and the norm of $x$ is equal to the norm of $y$ by $a x^{2}=-y^{2}$ and $a^{2}=-1$. Thus the norm of $2 x y$ is $\sqrt{2}$. Moreover, the norm of $\frac{a-1}{a+1}$ is 1 , as $a= \pm i$. Thus the norm of $2 x y$ is not equal to the norm of $\frac{a-1}{a+1}$, and are nonzero. So $f^{\times \times} \notin \mathscr{A}$ by Corollary 9.9.

This implies that $f^{\times}, f^{\times}$and $f^{\times \times}$cannot be all in $\mathscr{P}$, or all in $\mathscr{A}$, or all in $\widehat{\mathscr{M}}$. Thus the problem $\mathrm{Pl}-\# \operatorname{CSP}\left(f^{\times}, f^{\times}, f^{\times}\right)$is \#P-hard by Theorem 9.22. So $\mathrm{Pl}-\# \mathrm{CSP}^{2}(f)$ is \#P-hard.

Case 6: $\left[f_{0}, f_{2}, f_{4}\right]=[1, \pm 1,-1]$

In this case, $f=[1, x, 1, y,-1]$ or $[1, x,-1, y,-1]$. We consider the first case; the second case is similar.

We have $\partial(f)=[2, x+y, 0]$. If $x+y \neq 0$, then $\mathrm{Pl}-\# \operatorname{CSP}^{2}([2, x+y, 0])$ is \#P-hard by Theorem 9.21. Thus Pl-\#CSP $(f)$ is \#P-hard. Now we assume $x+y=0$. Next we apply Lemma 12.1 and calculate the determinant of the compressed matrix for $f$, which is a nonzero constant multiple of $x^{2}+1$. If $x^{2}+1 \neq 0$, then $\mathrm{Pl}_{-1} \mathrm{CSP}^{2}(f)$ is \#P-hard by Lemma 12.1.

If $x+y=0$ and $x^{2}+1=0$, then $f=[1, \pm i, 1, \mp i,-1]$. We have

$\partial(f)=2[1,0,0], \quad \partial_{[1,0,0]}(f)=[1, \pm i, 1], \quad$ and $\quad \partial_{[1, \pm i, 1]}(f)=[0, \pm 2 i, 2]$.

Then $\mathrm{Pl}$-\#CSP${ }^{2}([0, \pm 2 i, 2])$ is \#P-hard by Theorem 9.21. Thus $\mathrm{Pl}$-\#CSP${ }^{2}(f)$ is \#P-hard.

Case 7: $\left[f_{0}, f_{2}, f_{4}\right]=[1, b, 1]$ with $b^{2} \neq 0,1$

In this last case of Theorem 12.5, $f=[1, x, b, y, 1]$ and the determinant of the compress signature matrix is

$$
D=b+2 b x y-b^{3}-x^{2}-y^{2} .
$$

If $D \neq 0$, then $\mathrm{Pl}-\# \mathrm{CSP}^{2}(f)$ is \#P-hard by Lemma 12.1. In the following we assume that $D=0$.

If $x=y=0$, then $b=0$ or $b^{2}=1$ by $D=b\left(1-b^{2}\right)=0$. This is a contradiction. If $x=y \neq 0$, then $D=(1-b)\left[b(1+b)-2 x^{2}\right]=0$. By $b \neq 1$, we have $b(1+b)=2 x^{2}$. This implies that $f \in \widehat{\mathscr{M}}$ by Corollary 9.18.

Similarly, if $x=-y \neq 0$, then $D=(1+b)\left[b(1-b)-2 x^{2}\right]=0$. By $b \neq-1$, we have $b(1-b)=2 x^{2}$. This implies that $f \in \widehat{\mathscr{M}^{\dagger}}$ by Corollary 9.18.

In the following, assume that $x^{2} \neq y^{2}$ in additioto $D=0$. In this case, the "Three Stooges" are

$$
\begin{aligned}
f^{\times} & =[1, b, 1], \\
f^{\times} & =\left[1+b^{2}+2 x^{2}, 2 b^{2}+x^{2}+y^{2}, 1+b^{2}+2 y^{2}\right], \\
f^{\times \times} & =\left[1+b^{2}+2 x^{2}, 2 b+2 x y, 1+b^{2}+2 y^{2}\right] .
\end{aligned}
$$

and 
We will prove that $\operatorname{Pl}$-\#CSP $\left(f^{\times}, f^{\times}, f^{\infty \times}\right)$ is \#P-hard by showing that $f^{\times}, f^{\times}, f^{\times \times}$cannot all be in the same $\mathscr{P}$, or $\mathscr{A}$, or $\widehat{\mathscr{M}}$.

By $b^{2} \neq 0,1$, we have $f^{\times} \notin \mathscr{P}$ by Lemma 9.8 .

- Suppose $b^{2} \neq-1$. Then in addition to $b^{2} \neq 0,1$, we have $b^{4} \neq 0,1$. Then $f^{\times} \notin \mathscr{A}$ by Lemma 9.8. Moreover, if $f^{\triangleright} \in \widehat{\mathscr{M}}$, then by Lemma 9.8 and the fact that $x^{2} \neq y^{2}$, we must have

$$
1+b^{2}+2 x^{2}=-\left(1+b^{2}+2 y^{2}\right) \quad \text { and } \quad 2 b^{2}+x^{2}+y^{2}=0 .
$$

From (12.25), we get $b^{2}=1$. This is a contradiction. This implies that $f^{\times}, f^{\ngtr}$ cannot be all in $\mathscr{P}$, or all in $\mathscr{A}$, or all in $\widehat{\mathscr{M}}$ when $b^{2} \neq-1$.

- Now suppose $b^{2}=-1$. Then $f^{\ngtr}=\left[2 x^{2}, x^{2}+y^{2}-2,2 y^{2}\right]$ and $f^{\not x}=$ $2\left[x^{2}, b+x y, y^{2}\right]$. If $f^{x} \in \widehat{\mathscr{M}}$, then by $x^{2} \neq y^{2}$ and Lemma 9.8, we have

$$
x^{2}=-y^{2} \quad \text { and } \quad x^{2}+y^{2}-2=0
$$

This is a contradiction.

Finally suppose $\left\{f^{\not}, f^{\times \times}\right\} \subseteq \mathscr{A}$.

- If $x^{2}+y^{2}=0$, then $x y=-1$ by $b^{2}=-1$ and

$$
D=b+2 b x y-b^{3}-x^{2}-y^{2}=0 .
$$

Then $f^{\ngtr}=2\left[x^{2},-1, y^{2}\right], f^{\times \times}=2\left[x^{2}, b-1, y^{2}\right]$ both have all nonzero entries. If they are both in $\mathscr{A}$, the norm of their entries must be all the same $|b-1|=\left|x^{2}\right|=|-1|=1$, by Corollary 9.9. However $b-1$ does not have norm 1 since $b^{2}=-1$.

- If $x^{2}+y^{2} \neq 0$, then, since we also have $x^{2} \neq y^{2}$, the first and the last entries of both $f^{\ngtr}$ and $f^{\times}$are neither equal nor negative of each other. It follows from membership in $\mathscr{A}$ that $x^{2}+y^{2}-2=0$ and $b+x y=0$ by Corollary 9.9. Then by $D=b+2 b x y-b^{3}-x^{2}-y^{2}=$ 0 and $b^{2}=-1$, we get a contradiction.

We have proved that $f^{\times}, f^{\times}, f^{\times \times}$cannot be all in $\mathscr{P}$, or all in $\mathscr{A}$, or all in $\widehat{\mathscr{M}}$ when $b^{2}=-1$.

From above, $f^{\times}, f^{\times}, f^{\times \times}$cannot be all in $\mathscr{P}$, or all in $\mathscr{A}$, or all in $\widehat{\mathscr{M}}$ when $x^{2} \neq y^{2}$ and $D=0$. Thus $\mathrm{Pl}$-\#CSP $\left(f^{\times}, f^{\times}, f^{\times \times}\right)$is \#P-hard by Theorem 9.22. So $\mathrm{Pl}$-\#CSP${ }^{2}(f)$ is \#P-hard. This completes the proof of Case 7.

This completes the proof of Theorem 12.5. 


\section{An Application of Cyclotomic Field}

\subsection{Dichotomy Theorem with a Signature in $\widehat{\mathscr{M}} \backslash(\mathscr{P} \cup \widetilde{\mathscr{A}})$}

The next three lemmas are crucial. The purpose of these lemmas is to give a similar result as Lemma 11.3 when the signature set $\mathcal{F}$ contains some $f \in \widehat{\mathscr{M} \backslash} \backslash(\mathscr{P} \cup \widetilde{\mathscr{A}})$, and all signatures in $\mathcal{F}$ have even arity. The proof uses an argument involving the degree of extension of a cyclotomic field.

We first prove that if we have an even arity signature in $\widehat{\mathscr{M}} \backslash(\mathscr{P} \cup \tilde{\mathscr{A}})$, then we can construct a binary $[1, a, 1]$ with $a^{4} \notin\{0,1\}$.

Lemma 13.1 Let $\mathcal{F}$ be a set of symmetric signatures containing some $f \in \widehat{\mathscr{M}} \backslash$ $(\mathscr{P} \cup \widetilde{\mathscr{A}})$, which has even arity. Then

$$
\mathrm{Pl}-\# \mathrm{CSP}^{2}([1, a, 1], \mathcal{F}) \leq_{T} \mathrm{Pl}^{-\# C_{S P}^{2}}(\mathcal{F})
$$

for some a satisfying $a^{4} \notin\{0,1\}$.

Proof If $f$ has arity 2, then we are done by Lemma 9.14. Thus, we assume that $f$ has arity $2 n \geq 4$. By Lemma 9.14, we have either $f=[s, t]^{\otimes 2 n} \pm[t, s]^{\otimes 2 n}$ with $s^{4} \neq t^{4}$ and $s t \neq 0$ or $f_{k}=\epsilon^{k}(2 n-2 k)$ up to a scalar.

For $f=[s, t]^{\otimes 2 n}+[t, s]^{\otimes 2 n}$, we have $\partial^{n-1}(f)=\left(s^{2}+t^{2}\right)^{n-1}\left\{[s, t]^{\otimes 2}+\right.$ $\left.[t, s]^{\otimes 2}\right\}=\left(s^{2}+t^{2}\right)^{n}[1, a, 1]$, where $a=\frac{2 s t}{s^{2}+t^{2}}$. Note that $s^{2}+t^{2} \neq 0$ and $a \neq 0, \pm 1$. If $a \neq \pm i$, then we are done. Suppose $a= \pm i$. Then $g=\partial^{n-2}(f)=$ $\left(s^{2}+t^{2}\right)^{n-2}\left\{[s, t]^{\otimes 4}+[t, s]^{\otimes 4}\right\}$. A simple calculation shows that $g=-2 s^{2} t^{2}\left(s^{2}+\right.$ $\left.t^{2}\right)^{n-2}[3, \pm i,-1, \pm i, 3]$. Consider the gadget in Fig. 33. We assign $[3, \pm i,-1, \pm i, 3]$ to the circle vertices and $={ }_{6}$ to the square vertex. Its signature is $[8, \pm 6 i, 8]$, so we are done.

For $f=[s, t]^{\otimes 2 n}-[t, s]^{\otimes 2 n}$, we have $\partial^{n-1}(f)=\left(s^{2}+t^{2}\right)^{n-1}\left\{[s, t]^{\otimes 2}-\right.$ $\left.[t, s]^{\otimes 2}\right\}=\lambda[1,0,-1]$, where $\lambda=\left(s^{2}+t^{2}\right)^{n-1}\left(s^{2}-t^{2}\right) \neq 0$. For $2 n \geq 6$, we have $\partial_{[1,0,-1]}(f)=\left(s^{2}-t^{2}\right)\left\{[s, t]^{\otimes 2 n-2}+[t, s]^{\otimes 2 n-2}\right\}$ and we are done by the proof of the previous case, as $2 n-2 \geq 4$. For $2 n=4$, we have $\partial_{[1,0,-1]}(f)=$ $\left(s^{2}-t^{2}\right)\left\{[s, t]^{\otimes 2}+[t, s]^{\otimes 2}\right\}=\left(s^{4}-t^{4}\right)[1, a, 1]$, where $a=\frac{2 s t}{s^{2}+t^{2}} \neq 0, \pm 1$. If $a \neq \pm i$, then we are done. Suppose $a= \pm i$, then a simple calculation shows that $f$ is a nonzero multiple of $[2 i, \mp 1,0, \pm 1,-2 i]$. (One can verify that the ratio of the first two entries of $f=[s, t]^{\otimes 4}-[t, s]^{\otimes 4}$ is $\frac{s^{3} t-s t^{3}}{s^{4}-t^{4}}=\frac{s t}{s^{2}+t^{2}}=\frac{a}{2}= \pm \frac{i}{2}$.) Consider the gadget in Fig. 33. We assign $[2 i, \mp 1,0, \pm 1,-2 i]$ to the circle vertices and $={ }_{6}$ to the square vertex.. The signature of this gadget is $[-3, \mp 4 i,-3]$, so we are done.

For $f_{k}=\epsilon^{k}(2 n-2 k)$, we have $\partial^{n-2}(f)=2^{n-1}[2, \epsilon, 0,-\epsilon,-2]$. Consider the gadget in Fig. 33. We assign $[2, \epsilon, 0,-\epsilon,-2]$ to the circle vertices and $={ }_{6}$ to the square vertex. The signature of this gadget is $[5,4 \epsilon, 5]$, so we are done.

Fig. 33 Gadget used in the proof of Lemma 13.1

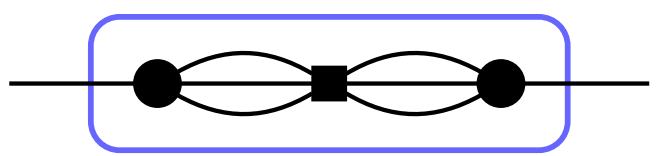


The next lemma shows that if we have $[1, a, 1]$ with $a^{4} \neq 0,1$, then we can obtain $[1,1]^{\otimes 2}$ by interpolation.

Lemma 13.2 For any signature set $\mathcal{F}$ and any $a^{4} \notin\{0,1\}$,

$$
\mathrm{Pl}-\# \operatorname{CSP}^{2}\left(\left\{[1,1]^{\otimes 2}\right\} \cup \mathcal{F}\right) \leq_{T} \mathrm{Pl}^{-\# C^{2}}{ }^{2}(\{[1, a, 1]\} \cup \mathcal{F}) .
$$

Proof The eigenvalues of $\left[\begin{array}{ll}1 & b \\ b & 1\end{array}\right]$ are $1+b$ and $1-b$ respectively. If we have a signature $[1, b, 1]$, for some $b \neq 1$, such that ratio $\frac{1+b}{1-b}$ of eigenvalues is not a root of unity, then we can interpolate any binary signature $[1, x, 1]$ for $x \in \mathbb{C}$. In particular, we can interpolate the desired $[1,1]^{\otimes 2}$.

Indeed, let $\Omega$ be an instance of $\operatorname{Pl}-\# \operatorname{CSP}^{2}(\{[1, x, 1]\} \cup \mathcal{F})$ in which $[1, x, 1]$ occurs $n$ times. Write $\left[\begin{array}{ll}1 & x \\ x & 1\end{array}\right]$ as $H\left[\begin{array}{ll}1+x & 0 \\ 0 & 1-x\end{array}\right] H$, where $H=\frac{1}{\sqrt{2}}\left[\begin{array}{ll}1 & 1 \\ 1 & -1\end{array}\right]$. We can stratify the partition function value on $\Omega$ as $\mathrm{Z}(\Omega)=\sum_{\ell=1}^{n} c_{\ell}(1+x)^{\ell}(1-x)^{n-\ell}$, where $c_{\ell}$ is the sum, over all assignments that assign 00 to $\ell$ copies of $\left[\begin{array}{ll}1+x & 0 \\ 0 & 1-x\end{array}\right]$ and 11 to the remaining $n-\ell$ copies, of the product of evaluations of all other signatures from $\mathcal{F}$ and those copies of $H$. If we construct a sequence $\Omega_{k}$ of instances of $\mathrm{Pl}$ \#CSP${ }^{2}(\{[1, b, 1]\} \cup \mathcal{F})$, where we replace each occurrence of $[1, x, 1]$ by a chain of $k$ linked copies of $[1, b, 1]$, then since $\left[\begin{array}{ll}1 & b \\ b & 1\end{array}\right]^{k}=H\left[\begin{array}{ll}(1+b)^{k} & 0 \\ 0 & (1-b)^{k}\end{array}\right] H$, we have $\mathrm{Z}\left(\Omega_{k}\right)=(1-b)^{k n} \sum_{\ell=1}^{n} c_{\ell}\left(\frac{1+b}{1-b}\right)^{k \ell}$, for $0 \leq k \leq n$. This is a Vandermonde system of full rank, and we can solve for all $c_{\ell}$ and find the value $\mathrm{Z}(\Omega)$.

The simple gadget with two copies of $=2 k$ connected by $2 k-1$ parallel copies of $[1, a, 1]$ has signature $\left[1, a^{2 k-1}, 1\right]$. If there is some $k \geq 1$ such that $a^{2 k-1}=1$, then $\left[1, a^{2 k-1}, 1\right]=[1,1]^{\otimes 2}$, and we are done. Suppose $a^{2 k-1} \neq 1$ for all $k \geq 1$. Our key claim is that there exists a $k \geq 1$, depending only on $a$, such that $\frac{1+a^{2 k-1}}{1-a^{2 k-1}}$ is not a root of unity. Then we are done by the interpolation given above.

For a contradiction, assume that $\frac{1+a^{2 k-1}}{1-a^{2 k-1}}$ is a root of unity for all $k \geq 1$. For $k=1$, $\frac{1+a}{1-a}$ is some root of unity $e^{2 \pi i j / m}$, where $\operatorname{gcd}(j, m)=1$. Then $a \in \Phi_{m}=\mathbb{Q}\left(e^{2 \pi i / m}\right)$, the $m$-th cyclotomic field. Therefore $a^{2 k-1} \in \Phi_{m}$ as well for all $k \geq 1$. Furthermore, $\left|\frac{1+a}{1-a}\right|=1$, so $a$ is purely imaginary, i.e. $a=i h$ for some real $h \notin\{0, \pm 1\}$ since $a^{4} \notin\{0,1\}$. First we consider the case $0<|h|<1$. Then $a^{2 k-1}= \pm i h^{2 k-1}$ and $\lim _{k \rightarrow \infty} h^{2 k-1}=0$.

By assumption, for all $k \geq 1, \frac{1+a^{2 k-1}}{1-a^{2 k-1}}$ is some root of unity $e^{2 \pi i J / M}$ (in which $J$ and $M$ depend on $k$ ), where $0<|J|<M / 2$ with $\operatorname{gcd}(J, M)=1$. Then $e^{2 \pi i / M} \in$ $\Phi_{m}$ as well, so $\Phi_{M} \subseteq \Phi_{m}$. Note that $|\tan (\pi J / M)|=|h|^{2 k-1}$. Hence $|h|^{2 k-1} \geq$ $\tan (\pi / M) \geq \pi / M$. Thus $M \geq \pi /|h|^{2 k-1}$.

However, the $M$-th cyclotomic field $\Phi_{M}$ has degree of extension $\left[\mathbb{Q}\left(e^{2 \pi i / M}\right)\right.$ : $\mathbb{Q}]=\varphi(M)$, where $\varphi$ is the Euler totient function. We have a crude estimate $(\varphi(M))^{2} \geq M / 2$ : Let $M=\prod_{i} p_{i}^{e_{i}}$ be its prime factorization, where $p_{i}$ are primes and $e_{i} \geq 1$, then $\varphi(M)=\prod_{i} \varphi\left(p_{i}^{e_{i}}\right)=\prod_{i} p_{i}^{e_{i}-1}\left(p_{i}-1\right)$. For $p=p_{i} \geq 3$, if $e \geq 1$, 
then $(p-1)^{2}>p$ which implies that $\left(\varphi\left(p^{e}\right)\right)^{2}=p^{2 e-2}(p-1)^{2}>p^{e}$. For $p=2$, if $e \geq 1$, then $\left(\varphi\left(2^{e}\right)\right)^{2}=2^{2 e-2} \geq 2^{e} / 2$. We get the lower bound $(\varphi(M))^{2} \geq M / 2$ by multiplicativity of $\varphi$. Then it follows that $\lim _{M \rightarrow \infty} \varphi(M)=\infty$, which contradicts $\varphi(M) \leq \varphi(m)<\infty$.

The remaining case $|h|>1$ can be handled similarly. In fact, $\lim _{k \rightarrow \infty}\left|h^{2 k-1}\right|=$ $\infty$, and therefore $\lim _{k \rightarrow \infty} \tan ^{-1}\left(\left|h^{2 k-1}\right|\right)=\frac{\pi}{2}$, but for all $k \geq 1, \tan ^{-1}\left(\left|h^{2 k-1}\right|\right)<$ $\frac{\pi}{2}$. Let $\theta_{k}$ be the argument of the complex number $\frac{1+a^{2 k-1}}{1-a^{2 k-1}}=\frac{1 \pm i h^{2 k-1}}{1 \mp i h^{2 k-1}}$, where the sign depends on $2 k-1 \equiv 1$ or $-1 \bmod 4$. Thus the absolute value $\left|\theta_{k}\right|$ of the angle $\theta_{k}$ is strictly less than $\pi$ but approaches $\pi$ when $k \rightarrow \infty$. On the other hand, the order of this root of unity is at most $2(\varphi(m))^{2}$. Any such root of unity not equal to -1 must have angle at least $\frac{1}{4(\varphi(m))^{2}}$ away from $\pi$. This is a contradiction.

Combining Corollary 9.13, Lemma 10.3, Lemma 13.1 and Lemma 13.2, we have proved the following lemma.

Lemma 13.3 Let $\mathcal{F}$ be a set of signatures of even arities. If $\mathcal{F}$ contains some $f \in$ $\widehat{\mathscr{M} \backslash} \backslash(\mathscr{P} \cup \mathscr{\mathscr { A }})$, then $\mathrm{Pl}-\# \mathrm{CSP}^{2}(\mathcal{F})$ is \#P-hard unless $\mathcal{F} \subseteq \widetilde{\mathscr{M}}$.

\subsection{Dichotomy Theorem with a Signature in $\widehat{\mathscr{M}}^{\dagger} \backslash(\mathscr{P} \cup \widetilde{\mathscr{A}})$}

We would like to prove a corresponding statement to Lemma 13.3 after replacing the condition $f \in \widehat{\mathscr{M}} \backslash(\mathscr{P} \cup \widetilde{\mathscr{A}})$ by $f \in \widehat{\mathscr{M}}^{\dagger} \backslash(\mathscr{P} \cup \widetilde{\mathscr{A}})$. This corresponding statement is indeed true and is implied by Theorem 9.2, the final dichotomy theorem for Pl-\#CSP ${ }^{2}$. However, at this point leading up to the proof of Theorem 9.2, we are not able to prove it. Instead, we prove a weaker version, Lemma 13.7, in which $f$ is assisted by a binary signature other than a multiple of $[1,0,1]$.

Remark 6 Here we explain some of the difficulties in the proof caused by structural complications of the signatures involved.

When we prove the No-Mixing statements for $\widehat{\mathscr{M}}$ the crucial step is the ability to construct $[1, \xi]^{\otimes 2}$ with $\xi \neq 0$ in the Pl-\#CSP${ }^{2}$ setting (cf. Lemma 13.1 and Lemma 13.2). This is the key, and the only known method, for us to leverage the existing dichotomy for Pl-\#CSP (cf. Lemma 10.3). Then in a similar spirit, to prove the No-Mixing statements for $\widehat{\mathscr{M}^{\dagger}}$, we would like to be able to construct $[1, \xi]^{\otimes 2}$ as well.

A signature $f=\left[f_{0}, \ldots, f_{n}\right]$ is called an odd signature if $f_{2 k}=0$ for all $k \geq 0$, and an even signature if $f_{2 k+1}=0$ for all $k \geq 0$.

In any $\mathcal{F}$-gate $H$, if every signature in $\mathcal{F}$ satisfies the parity constraints, then the signature of $H$ also satisfies the parity constraints. In fact the parity of the signature of $H$ is the same as the parity of the number of occurrences of odd signatures of $\mathcal{F}$ in $H$. To see this, suppose $\sigma$ is a $\{0,1\}$-assignment to all the edges of $H$, including internal and external edges, that has a nonzero evaluation on $H$. By the parity constraints, each odd (resp. even) signature appearing in $H$ has an odd (resp. even) number of incident edges assigned 1 . Adding up all these numbers mod 2, noting that each internal edge of $H$ assigned 1 contributes 2 to the sum while each external edge of $H$ assigned 1 contributes 1 , we get $N \equiv 2 X+Y \equiv Y(\bmod 2)$, where $N$ is the number of occurrences of odd signatures of $\mathcal{F}$ in $H$, and $X$ (resp. $Y$ ) is the 
number of internal (resp. external) edges assigned to 1 by $\sigma$. Hence $H$ has the same parity as $N$.

For any signature of the form $f=[s, t i]^{\otimes m} \pm[t, s i]^{\otimes m}$, or $f_{k}=(\epsilon i)^{k}(m-2 k)$, for any arity $m,\left(Z^{-1}\right)^{\otimes m} f$ satisfies the parity constraints, where $Z=\frac{1}{2}\left[\begin{array}{ll}1 & 1 \\ i & -i\end{array}\right]$. In fact for $f$ of the first type, $\left(Z^{-1}\right)^{\otimes m} f=[u, v]^{\otimes m} \pm[u,-v]^{\otimes m}$ for $u=s+t$ and $v=s-t$, and for $f$ of the second type, $\left(Z^{-1}\right)^{\otimes m} f=2^{m}[0,1,0, \ldots, 0]$ or $2^{m}[0, \ldots, 0,1,0]$. Note that

$$
\left[\begin{array}{ll}
1 & 1 \\
i & -i
\end{array}\right]^{\otimes m}[0,1,0, \ldots, 0]=\operatorname{Sym}_{m}^{m-1}\left(\left[\begin{array}{l}
1 \\
i
\end{array}\right] ;\left[\begin{array}{l}
1 \\
-i
\end{array}\right]\right)
$$

has its $k$-th term $i^{k}(m-2 k)$. Similarly, $\left[\begin{array}{ll}1 & 1 \\ i & -i\end{array}\right]^{\otimes m}[0, \ldots, 0,1,0]$ has its $k$-th term $(-i)^{k}(m-2 k)$.

Under the holographic transformation $Z$, we have

$$
\operatorname{Pl-\# CSP}(f) \equiv_{T} \operatorname{Pl}-H o l a n t([0,1,0],[1,0,1,0,1], \ldots \mid \hat{f}),
$$

where $\hat{f}=\left(Z^{-1}\right)^{\otimes m} f$, and $\frac{1}{2}[0,1,0]=(=2) Z^{\otimes 2}, \frac{1}{2^{3}}[1,0,1,0,1]=\left(={ }_{4}\right) Z^{\otimes 4}$, etc. Notice that for the signatures $(=2 n) Z^{\otimes 2 n}$, if the arity $2 n \equiv 2(\bmod 4)$ then the signature is odd, and if $2 n \equiv 0(\bmod 4)$ then the signature is even.

Every signature of the form $[s, t i]^{\otimes m}+[t, s i]^{\otimes m}$ is even, every signature of the form $[s, t i]^{\otimes m}-[t, s i]^{\otimes m}$ is odd, and for even arity $2 n$ the signatures $[0,1,0, \ldots, 0]$ and $[0, \ldots, 0,1,0]$ are both odd.

Thus, if we focus on signatures $f=[s, t i]^{\otimes 2 n}+[t, s i]^{\otimes 2 n}$ with arity $2 n \equiv 0$ $(\bmod 4)$, or $f=[s, t i]^{\otimes 2 n}-[t, s i]^{\otimes 2 n}$ with arity $2 n \equiv 2(\bmod 4)$, or $f_{k}=$ $(\epsilon i)^{k}(2 n-2 k)$ with arity $2 n \equiv 2(\bmod 4)$, then the following property holds for all the signatures in the bipartite Pl-Holant problem in (13.26):

All signatures of arity $2 n \equiv 2(\bmod 4)$ satisfy odd parity and all signatures of arity $2 n \equiv 0(\bmod 4)$ satisfy even parity.

It follows that, for such $f$, any gadget constructed from (13.26) has the same parity as the number of occurrences of signatures of arity $2 n \equiv 2(\bmod 4)$.

Furthermore, in a bipartite gadget construction in Pl-Holant $([0,1,0]$, $[1,0,1,0,1], \ldots \mid \hat{f})$, if the resulting signature of the gadget is binary, the number of occurrences of signatures of arity $2 n \equiv 2(\bmod 4)$ in this gadget must be odd. Indeed let $N_{0}$ (resp. $N_{2}$ ) denote the number of occurrences of signatures of arity $2 n \equiv 0(\bmod 4)(\operatorname{resp} .2 n \equiv 2(\bmod 4))$ in this bipartite gadget, and we add up the arities of all signatures modulo 4 , we get $0 N_{0}+2 N_{2} \equiv 2 N_{I}+2(\bmod 4)$, where $N_{I}$ is the number of internal edges in the bipartite gadget, and the additive term 2 is because the gadget is a binary gadget. Thus $N_{2} \equiv N_{I}+1(\bmod 2)$. On the other hand, since the gadget is bipartite, $N_{I}$ is the sum of all arities of signatures from RHS, and minus 2 if the external 2 edges come from the RHS. As all signatures in this gadget have even arity, $N_{I} \equiv 0(\bmod 2)$. Hence $N_{2} \equiv 1(\bmod 2)$. 
This implies that any binary signature constructed in Pl-Holant $([0,1,0]$, $[1,0,1,0,1], \ldots \mid \hat{f})$ must have odd parity, i.e., they are all of the form $\lambda[0,1,0]$. Thus, before the $Z$-transformation, one can only construct binary signatures of the form $\frac{\lambda}{2}[1,0,1]=\lambda Z^{\otimes 2}[0,1,0]$ in Pl-\#CSP $(f)$ by gadget construction. This can be verified as $\left[\begin{array}{ll}1 & 1 \\ i & -i\end{array}\right]\left[\begin{array}{ll}0 & 1 \\ 1 & 0\end{array}\right]\left[\begin{array}{ll}1 & i \\ 1 & -i\end{array}\right]=2\left[\begin{array}{ll}1 & 0 \\ 0 & 1\end{array}\right]$.

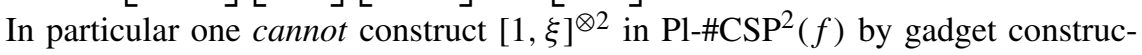
tion. This explains the extra mile we have to travel in this proof.

As indicated, therefore, we prove a weaker version of Lemma 13.3 in this subsection, namely Lemma 13.7 , in which $f$ is assisted by a binary signature other than a multiple of $[1,0,1]$.

We begin with the following lemma.

Lemma 13.4 Let $\mathcal{F}$ be any set of symmetric signatures of even arities, and suppose $\mathcal{F}$ contains signatures $f$ and $g$, where $f \in \widehat{\mathscr{M}}^{\dagger} \backslash(\mathscr{P} \cup \mathscr{A})$, and $g=\left[g_{0}, g_{1}, \ldots, g_{2 n}\right]$ and there exists a positive integer s such that $g_{0}^{s}=-g_{2 n}^{s} \neq 0$. Then either $\mathcal{F} \subseteq \mathscr{M}^{\dagger}$ or $\mathrm{Pl}$-\#CSP${ }^{2}(\mathcal{F})$ is \#P-hard.

Proof Let $E_{2 k}(-1)=[1,0, \ldots, 0,-1]$ have arity $2 k$ and $\mathcal{E}(-1)=\left\{E_{2 k}(-1) \mid k \geq\right.$ $1\}$. Firstly, by our calculus we have $\partial_{g}^{s}\left(=_{2 n s+2 k}\right)=g_{0}^{s} E_{2 k}(-1)$ on LHS for $k \geq 1$. Thus we have

$$
\operatorname{Pl} \text {-Holant }\left(\mathcal{E}(-1) \cup \mathcal{E} \mathcal{Q}_{2} \mid \mathcal{F}\right) \leq_{T} \operatorname{Pl}-\# \operatorname{CSP}^{2}(\mathcal{F})
$$

Under a holographic transformation by $T^{-1}=\left[\begin{array}{ll}1 & 0 \\ 0 & i\end{array}\right]$, the set $\mathcal{E}(-1) \cup \mathcal{E} \mathcal{Q}_{2}$ is setwise invariant. Indeed, for all $k \geq 1$, signatures of arity $4 k$ in $\mathcal{E}(-1) \cup \mathcal{E} \mathcal{Q}_{2}$ are pointwise fixed, and signatures of arity $4 k-2$ in $\mathcal{E}(-1)$ and in $\mathcal{E} \mathcal{Q}_{2}$ are interchanged. Thus, $\operatorname{Pl}-\# \operatorname{CSP}^{2}(T \mathcal{F}) \leq_{T} \operatorname{Pl-Holant}\left(\mathcal{E}(-1) \cup \mathcal{E} \mathcal{Q}_{2} \mid T \mathcal{F}\right) \equiv_{T} \operatorname{Pl-Holant}\left(\mathcal{E}(-1) \cup \mathcal{E} \mathcal{Q}_{2} \mid \mathcal{F}\right)$. Note that $T^{\otimes 2 n} f \in T \mathcal{F}$ is in $\widehat{\mathscr{M} \backslash}(\mathscr{P} \cup \widetilde{\mathscr{A}})$. Thus either $T \mathcal{F} \subseteq \widehat{\mathscr{M}}$ or $\operatorname{Pl}_{-} \operatorname{CSP}^{2}(T \mathcal{F})$ is \#P-hard by Lemma 13.3. Note that $T \mathcal{F} \subseteq \widehat{\mathscr{M}}$ iff $\mathcal{F} \subseteq \widehat{\mathscr{M}^{\dagger}}$. Thus either $\mathcal{F} \subseteq \widehat{\mathscr{M}^{\dagger}}$ or $\mathrm{Pl}-\# \mathrm{CSP}^{2}(\mathcal{F})$ is \#P-hard.

The next two lemmas show that if we have a signature in $\widehat{\mathscr{M}} \backslash \backslash(\mathscr{P} \cup \widetilde{\mathscr{A}})$ and a binary signature that is not a multiple of $[1,0,1]$, then we have the same statement for $\widetilde{\mathscr{M}}^{\dagger}$, as Lemma 13.3 is for $\widehat{\mathscr{M}}$. This will be stated as Lemma 13.7. Note that if $f \in \widehat{\mathscr{M}} \backslash(\mathscr{P} \cup \widetilde{\mathscr{A}})$ is a binary signature, then $f$ takes the form $[1, b,-1]$ by Lemma 9.14, and this case is covered by Lemma 13.4, where $f$ also plays the role of $g$. Thus we assume $f \in \widehat{\mathscr{M}}^{\dagger} \backslash(\mathscr{P} \cup \widetilde{\mathscr{A}})$ has arity $\geq 4$. By Lemma 9.14 , such a signature $f$ has two forms. Lemma 13.5 and 13.6 handle these two cases respectively.

Lemma 13.5 Let $\mathcal{F}$ be any set of symmetric signatures of even arities, and suppose $\mathcal{F}$ contains signatures $f$ and $h$, where $f=[s, t i]^{\otimes 2 n} \pm[t, s i]^{\otimes 2 n}$ with $2 n \geq 4$, $s^{4} \neq t^{4}$ and $s t \neq 0$, and $h$ is any nonzero binary signature other than $\lambda[1,0,1]$. Then either $\mathcal{F} \subseteq \widehat{\mathscr{M}}^{\dagger}$ or $\mathrm{Pl}$-\#CSP${ }^{2}(\mathcal{F})$ is \#P-hard. 
Proof Firstly, by our calculus, ignoring the nonzero factor $\left(s^{2}-t^{2}\right)^{n-2}$ in $\partial^{n-2}(f)$, we have $g=[s, t i]^{\otimes 4} \pm(-1)^{n-2}[t, s i]^{\otimes 4}$. If $g=[s, t i]^{\otimes 4}-[t, s i]^{\otimes 4}$, then we have $\partial(g)=\left(s^{2}-t^{2}\right)\left\{[s, t i]^{\otimes 2}+[t, s i]^{\otimes 2}\right\}=\left(s^{2}-t^{2}\right)\left[s^{2}+t^{2}, 2 s t i,-\left(s^{2}+t^{2}\right)\right]$ and we are done by Lemma 13.4 .

Suppose $g=[s, t i]^{\otimes 4}+[t, s i]^{\otimes 4}$, and we also have $h \neq \lambda[1,0,1]$. If $h \notin \mathscr{P} \cup$ $\widetilde{\mathscr{A}} \cup \widetilde{\mathscr{M}}$, then $\mathrm{Pl}$-\#CSP${ }^{2}(\mathcal{F})$ is \#P-hard by Theorem $9.21^{\prime}$. Otherwise, by Lemma 9.8, the possibilities for $h$, after normalizing, are

$[a, b]^{\otimes 2},[1,0, x],[0,1,0],\left[1, \rho,-\rho^{2}\right],\left[1, \alpha,-\alpha^{2}\right],[1, u, 1]$, and $[1, v,-1]$, where $x \notin\{0,1\}, \rho^{4}=1, \alpha^{4}=-1, u^{4} \notin\{0,1\}$, and $v^{4} \notin\{0,1\}$.

- If $h=[a, b]^{\otimes 2}$ with $a b \neq 0$, then we are done by Lemma 10.3.

- If $h \in\left\{[1,0,-1],[1,0, \pm i],[1, \pm 1,-1],\left[1, \alpha,-\alpha^{2}\right],[1, v,-1]\right\}$, then we are done by Lemma 13.4.

- If $h=[1, u, 1]$ with $u^{4} \neq 0,1$, then $h \in \widehat{\mathscr{M} \backslash} \backslash(\mathscr{P} \cup \widetilde{\mathscr{A}})$ by Lemma 9.14. Thus we are done by Lemma 13.3.

The remaining cases are $h=[1,0]^{\otimes 2},[0,1]^{\otimes 2},[1,0, x],[0,1,0]$ or $[1, \pm i, 1]$, where $x^{4} \neq 0,1$.

- If $h=[1,0, x]$ with $x^{4} \neq 0,1$, then by taking 4 copies of $h$ and connecting one input of $h$ to each edge of $g$, we have $\hat{g}=\left[\begin{array}{ll}1 & 0 \\ 0 & x\end{array}\right]^{\otimes 4} g=[s, x t i]^{\otimes 4}+$ $[t, x s i]^{\otimes 4}$. The signature $\hat{g}$ is non-degenerate, has arity 4 , and satisfies a second order recurrence relation. The eigenvalues of the recurrence relation are $\frac{x t i}{s}$ and $\frac{x s i}{t}$. By the trace and product, $\hat{g}$ has type $\left\langle-x^{2}, \frac{x t i}{s}+\frac{x s i}{t}, 1\right\rangle$. Thus $\hat{g} \notin \mathscr{P} \cup$ $\widetilde{\mathscr{A}} \cup \widetilde{\mathscr{M}}$ by Lemma 9.11, since $\left(-x^{2}\right)^{2} \neq 0,1$ and $\frac{t}{s}+\frac{s}{t} \neq 0 . \operatorname{So~Pl}^{2} \# \operatorname{CSP}^{2}(\hat{g})$ is \#P-hard by Theorem 12.5. Thus $\mathrm{Pl}-\# \mathrm{CSP}^{2}(\mathcal{F})$ is \#P-hard.

- If $h=[0,1,0]$, then $\partial_{h}(g)=2 s t i\left\{[s, t i]^{\otimes 2}+[t, s i]^{\otimes 2}\right\}=2 s t i\left[s^{2}+\right.$ $\left.t^{2}, 2 s t i,-\left(s^{2}+t^{2}\right)\right]$. Then we are done by Lemma 13.4.

- If $h=[1, \pm i, 1]$, by connecting two copies of $[1, \pm i, 1]$ we have $\pm 2 i[0,1,0]$, as $\left[\begin{array}{ll}1 & \pm i \\ \pm i & 1\end{array}\right]^{2}=\left[\begin{array}{ll}0 & \pm 2 i \\ \pm 2 i & 0\end{array}\right]$. Then we are done by the previous case.

- If $h=[1,0]^{\otimes 2}$, then we have $g^{\prime}=\partial_{h}(g)=s^{2}[s, t i]^{\otimes 2} \pm t^{2}[t, s i]^{\otimes 2}=\left[s^{4}+\right.$ $\left.t^{4},\left(s^{2}+t^{2}\right) s t i,-2 s^{2} t^{2}\right]$. We claim that $g^{\prime} \notin \mathscr{P} \cup \widetilde{\mathscr{A}} \cup \widetilde{\mathscr{M}}$.

- If $g^{\prime} \in \mathscr{P}$, then $g^{\prime}$ is degenerate by $\left(s^{2}+t^{2}\right) s t i \neq 0$ and $-2 s^{2} t^{2} \neq 0$. So $-2 s^{2} t^{2}\left(s^{4}+t^{4}\right)=-\left(s^{2}+t^{2}\right)^{2} s^{2} t^{2}$. Thus $s t=0$ or $\left(s^{2}-t^{2}\right)^{2}=0$. This is a contradiction.

- If $g^{\prime} \in \mathscr{A} \backslash \mathscr{P}$, then $g^{\prime}=\left[1, \rho,-\rho^{2}\right]$ up to a scalar by Corollary 9.9, where $\rho^{4}=1$. By $\rho^{2}= \pm 1$, we have $s^{4}+t^{4}= \pm 2 s^{2} t^{2}$. This contradicts that $s^{4} \neq t^{4}$.

- If $g^{\prime} \in \mathscr{A}^{\dagger} \backslash \mathscr{P}$, then $g^{\prime}=\left[1, \alpha,-\alpha^{2}\right]$ up to a scalar by Corollary 9.9, where $\alpha^{4}=-1$. Thus $2 s^{2} t^{2}\left(s^{4}+t^{4}\right)=-\left(s^{2}+t^{2}\right)^{2} s^{2} t^{2}$. Then, by $s t \neq 0$, we have $3\left(s^{4}+t^{4}\right)=-2 s^{2} t^{2} \neq 0$, and so $\left|s^{4}+t^{4}\right| \neq\left|-2 s^{2} t^{2}\right|$. This implies that the norms of two nonzero entries of $g^{\prime}$ are not equal. This contradicts the form $g^{\prime}=\lambda\left[1, \alpha,-\alpha^{2}\right]$. 
- Since $s^{4} \neq t^{4}$ we have $s^{4}+t^{4} \neq \pm 2 s^{2} t^{2}$. Hence $g^{\prime} \notin \widetilde{\mathscr{M}}$ by Corollary 9.9 .

Then by Theorem 9.21', Pl-\#CSP${ }^{2}\left(g^{\prime}\right)$ is \#P-hard. Thus $\mathrm{Pl}-\# \operatorname{CSP}^{2}(\mathcal{F})$ is \#P-hard. - If $h=[0,1]^{\otimes 2}$, then we apply the transformation $\left[\begin{array}{ll}0 & 1 \\ 1 & 0\end{array}\right]$ and are done by the previous case.

Lemma 13.6 Let $\mathcal{F}$ be any set of symmetric signatures of even arities, and suppose $\mathcal{F}$ contains signatures $f$ and $h$, where $f$ has arity $2 n \geq 4$ and $f_{k}=(\epsilon i)^{k}(2 n-2 k)$, and $h$ is any nonzero binary signature other than $\lambda[1,0,1]$. Then either $\mathcal{F} \subseteq \widehat{\mathscr{M}}^{\dagger}$ or $\mathrm{Pl}$ \# $\operatorname{CSP}^{2}(\mathcal{F})$ is \#P-hard.

Proof If $2 n \equiv 0(\bmod 4)$, then $f_{0}=-f_{2 n}=2 n$. Thus we are done by Lemma 13.4. Suppose $2 n \equiv 2(\bmod 4)$. Thus $n \geq 3$ and we have $g=\partial_{=4}^{\frac{n-3}{2}}(f)$ of arity 6 . Ignoring the nonzero factor $2^{\frac{n-3}{2}}$, we have $g_{k}=(\epsilon i)^{k}(6-2 k)$. Removing another factor 2, we have

$$
g=[3,2 i \epsilon,-1,0,-1,-2 i \epsilon, 3] .
$$

We also have a nonzero binary signature $h \neq \lambda[1,0,1]$. If $h \notin \mathscr{P} \cup \widetilde{\mathscr{A}} \cup \widetilde{\mathscr{M}}$, then $\mathrm{Pl}$-\#CSP${ }^{2}(\mathcal{F})$ is \#P-hard by Theorem 9.21'. Otherwise (similar to the proof of Lemma 13.5), by Lemma 9.8, the possibilities for $h$, after normalizing, are

$[a, b]^{\otimes 2},[1,0, x],[0,1,0],\left[1, \rho,-\rho^{2}\right],\left[1, \alpha,-\alpha^{2}\right],[1, u, 1], \quad$ and $[1, v,-1]$, where $x \notin\{0,1\}, \rho^{4}=1, \alpha^{4}=-1, u^{4} \notin\{0,1\}$, and $v^{4} \notin\{0,1\}$. If $h=[1,0,-1]$, $[1,0, \pm i],[1, \pm 1,-1],\left[1, \alpha,-\alpha^{2}\right],[1, v,-1],[1, u, 1]$, or $[a, b]^{\otimes 2}$ with $a b \neq 0$, then we are done with the same proof as in Lemma 13.5.

The remaining cases are $h=[1,0]^{\otimes 2},[0,1]^{\otimes 2},[1,0, x],[0,1,0]$, or $[1, \pm i, 1]$, where $x^{4} \notin\{0,1\}$.

- For $h=[1,0, x]$ with $x^{4} \notin\{0,1\}$, we have $g^{\prime}=\partial_{h}(g)=[3-x, \pm 2 i,-1-$ $x, \mp 2 x i,-1+3 x]$. The signature $g^{\prime}$ is non-degenerate because $( \pm 2 i)(\mp 2 x i) \neq$ $(-1-x)^{2}$ by $x \neq 1$. Moreover, $g^{\prime}$ satisfies the second order recurrence relation with type $\langle 1, \mp 2 i,-1\rangle$. Thus $g^{\prime} \notin \mathscr{P} \cup \widetilde{\mathscr{A}} \cup \widehat{\mathscr{M}}$ by Lemma 9.11. Moreover, by $x \neq \pm 1$, we have $3-x \neq \pm(-1+3 x)$, so $g^{\prime} \notin \widehat{\mathscr{M}^{\dagger}}$ by Corollary 9.17. So $\mathrm{Pl}-\# \operatorname{CSP}^{2}\left(g^{\prime}\right)$ is \#P-hard by Theorem 12.5. Thus $\operatorname{Pl} \# \operatorname{CSP}^{2}(\mathcal{F})$ is \#P-hard.

- If $h=[0,1,0]$, then $\partial_{h}(g)=[ \pm 2 i,-1,0,-1, \pm 2 i]$. Then we are done by Lemma 13.4.

- If $h=[1, \pm i, 1]$, by connecting two copies of $[1, i, \pm 1]$ we have $\pm 2 i[0,1,0]$. Then we are done by the proof of the previous case.

- If $h=[1,0]^{\otimes 2}$, then we have $g^{\prime \prime}=\partial_{h}^{2}(g)=[3, \pm 2 i,-1]$. By Corollary 9.9, we have $g^{\prime \prime} \notin \mathscr{P} \cup \widetilde{\mathscr{A}} \cup \widetilde{\mathscr{M}}$. Then by Theorem $9.21^{\prime}, \mathrm{Pl}-\# \operatorname{CSP}^{2}\left(g^{\prime \prime}\right)$ is \#P-hard. Thus $\mathrm{Pl}$ \#\#CSP${ }^{2}(\mathcal{F})$ is \#P-hard.

- If $h=[0,1]^{\otimes 2}$, we apply the transformation $\left[\begin{array}{ll}0 & 1 \\ 1 & 0\end{array}\right]$ and it follows from the previous case. 
Lemma 13.7 Let $\mathcal{F}$ be any set of symmetric signatures of even arities, and suppose $\mathcal{F}$ contains signatures $f$ and $h$, where $f \in \mathscr{\mathscr { M }}^{\dagger} \backslash(\mathscr{P} \cup \mathscr{\mathscr { A }})$, and $h$ is any nonzero binary signature other than $\lambda[1,0,1]$. Then either $\mathcal{F} \subseteq \widehat{\mathscr{M}}^{\dagger}$ or $\operatorname{Pl}-\# \operatorname{CSP}^{2}(\mathcal{F})$ is \#P-hard.

Proof If $f$ has arity 2 , then $f=[1, b,-1]$ by Lemma 9.14 . Then we are done by Lemma 13.4.

If $f$ has arity $2 n \geq 4$, then by Lemma 9.14, we have $f=[s, t i]^{\otimes 2 n} \pm[t, s i]^{\otimes 2 n}$ with $s t \neq 0, s^{4} \neq t^{4}$, or $f_{k}=(\epsilon i)^{k}(2 n-2 k)$ up to a scalar. These two cases are proved in Lemma 13.5, and 13.6 respectively.

Remark 7 Lemma 13.3 and Lemma 13.7 will substantially simplify the succeeding proof for No-Mixing Lemmas concerning $\widehat{\mathscr{M}}$ and $\widehat{\mathscr{M}}{ }^{\dagger}$. Thus it is natural that we wish to do the same for $\mathscr{A}$, and that means we would like to construct $[1, \xi]^{\otimes 2}$ with $\xi \neq 0$ in $\mathrm{Pl}-\# \operatorname{CSP}^{2}(f)$ for $f \in \mathscr{A}$. Unfortunately, for most cases of $f \in \mathscr{A}$ this is impossible.

First, for a signature $f \in \mathscr{A}$, if $f$ satisfies the parity constraints, then all signatures constructed in $\mathrm{Pl}$-\#CSP${ }^{2}(f)$ satisfy the parity constraints, since all $\mathcal{E} \mathcal{Q}_{2}$ also satisfy the parity constraints. So it is impossible to construct $[1, \xi]^{\otimes 2}$ with $\xi \neq 0$ in $\mathrm{Pl}-\# \mathrm{CSP}^{2}(f)$.

If a signature $f \in \mathscr{A}$ is degenerate and does not satisfy the parity constraints, then $f=[1, \pm 1]^{\otimes 2 n}$ or $f=[1, \pm i]^{\otimes 2 n}$ up to a scalar. For $f=[1, \pm 1]^{\otimes 2 n}$, we have $\partial^{n-1}(f)=2^{n-1}[1, \pm 1]^{\otimes 2}$. For $f=[1, \pm i]^{\otimes 2 n}$ and $2 n \equiv 2(\bmod 4)$, we have $\partial_{=}^{\frac{n-1}{2}}(f)=2^{\frac{n-1}{2}}[1, \pm i]^{\otimes 2}$. Thus in these two particular cases we can get $[1, \xi]^{\otimes 2}$ with $\xi \neq 0$. We will show that these are the only cases that this is possible.

Let $f=[1, \pm i]^{\otimes 2 n}$ and $2 n \equiv 0(\bmod 4)$. After a holographic transformation by $Z=\left[\begin{array}{ll}1 & 1 \\ i & -i\end{array}\right]$, we have

$$
\mathrm{Pl}-\# \operatorname{CSP}^{2}(f) \equiv_{T} \text { Pl-Holant}([0,1,0],[1,0,1,0,1], \ldots \mid \hat{f}),
$$

where $\hat{f}=\left(Z^{-1}\right)^{\otimes 2 n} f$, i.e., $\hat{f}=[1,0]^{\otimes 2 n}$ or $\hat{f}=[0,1]^{\otimes 2 n}$. In Pl-Holant $([0,1,0],[1,0,1,0,1], \ldots \mid \hat{f})$, all signatures of arity $\equiv 0(\bmod 4)$ have even parity and all signatures of arity $\equiv 2(\bmod 4)$ have odd parity. By the same proof in Remark 6, all nonzero binary signatures that can be constructed in Pl-Holant $([0,1,0],[1,0,1,0,1], \ldots \mid \hat{f})$ are multiples of $[0,1,0]$. In terms of signatures that can be constructed before the $Z$-transformation, this is equivalent to say that all nonzero binary signatures that can be constructed in $\operatorname{Pl}_{-1} \operatorname{CSP}^{2}(f)$ must be multiples of $[1,0,1]$. In particular, one cannot construct $[1, \xi]^{\otimes 2}$ with $\xi \neq 0$ in $\mathrm{Pl}-\# \mathrm{CSP}^{2}(f)$.

If $f \in \mathscr{A}$ is non-degenerate and does not satisfy the parity constraints, then $f=$ $[1, i]^{\otimes 2 n} \pm i[1,-i]^{\otimes 2 n}$ or $f=[1,1]^{\otimes 2 n} \pm i[1,-1]^{\otimes 2 n}$. If we can construct $[1, \xi]^{\otimes 2}$ with $\xi \neq 0$ in $\operatorname{Pl}_{-\# C^{2}}(f)$, then $[1, \xi]^{\otimes 2}$ must be in $\mathscr{A}$. Thus $[1, \xi]^{\otimes 2}=[1, \pm 1]^{\otimes 2}$ or $[1, \pm i]^{\otimes 2}$.

For $f=[1, i]^{\otimes 2 n} \pm i[1,-i]^{\otimes 2 n}, f=\left[1, \pm 1,-1, \mp 1, \ldots,(-1)^{n}\right]$ up to the scalar $1 \pm i$. In any construction in $\mathrm{Pl}_{-\# C^{2}}(f)$, if we ignore a global scalar factor which 
is a power of $1 \pm i$, all entries of the constructed signature are real numbers. Thus the ratio of any two nonzero entries is a real number. But this is not the case with $[1, \pm i]^{\otimes 2}$. This implies that we cannot construct $[1, \pm i]^{\otimes 2}$ in $\operatorname{Pl}_{-} \# \mathrm{CSP}^{2}(f)$ by gadget construction.

Moreover, we claim that it is impossible to get $[1, \pm 1]^{\otimes 2}$ in $\mathrm{Pl}-\# \operatorname{CSP}^{2}(f)$ by gadget construction. After a holographic transformation by $Z=\left[\begin{array}{ll}1 & 1 \\ i & -i\end{array}\right]$, we have

$$
\left.\operatorname{Pl-\# CSP}(f) \equiv_{T} \text { Pl-Holant([0, 1, 0], }[1,0,1,0,1], \ldots \mid \hat{f}\right),
$$

where $\hat{f}=\left(Z^{-1}\right)^{\otimes 2 n} f=[1,0, \ldots, 0, \pm i]$. All signatures in Pl-Holant $([0,1,0]$, $[1,0,1,0,1], \ldots \mid \hat{f})$ satisfy the parity constraints. Thus we cannot construct $\left(Z^{-1}\right)^{\otimes 2}[1, \pm 1]^{\otimes 2}=\mp \frac{i}{2}[1, \pm i]^{\otimes 2}$, which does not satisfy the parity constraints, by gadget construction. Thus we cannot get $[1, \pm 1]^{\otimes 2}$ in $\mathrm{Pl}-\# \mathrm{CSP}^{2}(f)$ by gadget construction.

$$
\text { For } f=[1,1]^{\otimes 2 n} \pm i[1,-1]^{\otimes 2 n} \text {, after a holographic transformation by }\left[\begin{array}{ll}
1 & 0 \\
0 & i
\end{array}\right] \text {, we }
$$

can use the same argument as the previous case for $[1, \pm i]^{\otimes 2}$ to prove that we cannot get $[1, \pm 1]^{\otimes 2}$ in $\mathrm{Pl}-\# \mathrm{CSP}^{2}(f)$ by gadget construction. Moreover, it is also impossible to get $[1, \pm i]^{\otimes 2}$ in $\mathrm{Pl}-\# \operatorname{CSP}^{2}(f)$ by gadget construction. After a holographic transformation by $H=\left[\begin{array}{ll}1 & 1 \\ 1 & -1\end{array}\right]$, we have

$$
\operatorname{Pl-\# CSP}(f) \equiv_{T} \operatorname{Pl-Holant}([1,0,1],[1,0,1,0,1], \ldots \mid \hat{f}),
$$

where $\hat{f}=\left(H^{-1}\right)^{\otimes 2 n} f=[1,0, \ldots, 0, \pm i]$. All signatures in Pl-Holant([1, 0, 1], $[1,0,1,0,1], \ldots \mid \hat{f})$ satisfy the parity constraints. Thus we cannot construct $\left(H^{-1}\right)^{\otimes 2}[1, \pm i]^{\otimes 2}= \pm \frac{i}{2}[1, \mp i]^{\otimes 2}$ by gadget construction. This implies that we cannot get $[1, \pm i]^{\otimes 2}$ in $\mathrm{Pl}-\# \mathrm{CSP}^{2}(f)$ by gadget construction.

\section{No-Mixing of a Pair of Signatures of Even Arity}

The general theme of this section and the next is that, for planar $\mathrm{Pl}-\# \mathrm{CSP}^{2}$ problems, various tractable signatures of different types cannot mix. In these two sections, all signatures are of even arity. In this section we prove a No-Mixing theorem for a pair of signatures. This will be extended to a set of signatures in the next section.

Recall that

$$
S_{1}=\widehat{\mathscr{M}}, \quad S_{2}=\widehat{\mathscr{M}^{\dagger}}, \quad S_{3}=\mathscr{A}^{\dagger}, \quad S_{4}=\mathscr{A}, \quad \text { and } \quad S_{5}=\mathscr{P} .
$$

The general form of the No-Mixing theorem to be proved in this section is as follows: Let $f$ and $g$ be two symmetric signatures of even arity. Suppose for some $1 \leq j<$ $i \leq 5, f \in S_{i} \backslash S_{j}$ and $g \in S_{j} \backslash S_{i}$, and for all $1 \leq k \leq 5,\{f, g\} \nsubseteq S_{k}$. Then $\mathrm{Pl}$-\#CSP${ }^{2}(f, g)$ is \#P-hard. We will call such a statement No-Mixing- $(i, j)$ (or NoMixing theorem- $(i, j)$, or a No-Mixing lemma). 
It is easy to see that, with possibly switching the names $f$ and $g$, the condition stated above is equivalent to the following assumption:

$$
\{f, g\} \subseteq \bigcup_{k=1}^{5} S_{k} \text { but for any } 1 \leq k \leq 5, \text { we have }\{f, g\} \nsubseteq S_{k} .
$$

However under this assumption, we make the following observation that any index $i$ for which $f \in S_{i}$ can be chosen as the distinguishing index:

If $f \in S_{i}$ for some $i$, then there exists some $j \neq i$ such that $g \in S_{j} \backslash S_{i}$ and $f \in S_{i} \backslash S_{j}$.

In particular, neither $f$ nor $g$ can be identically 0 .

We will prove the No-Mixing theorem- $(i, j)$ in a reverse lexicographic order of $(i, j)$ : We order the statements as $(5,4),(5,3),(5,2)$, $(5,1),(4,3),(4,2),(4,1),(3,2),(3,1),(2,1)$. After having proved all No-Mixing theorem- $\left(i^{\prime}, j^{\prime}\right)$ preceding $(i, j)$ in this order, we assume there are two signatures $f$ and $g$ such that $f \in S_{i} \backslash S_{j}$ and $g \in S_{j} \backslash S_{i}$. Now we may make the following additional assumption:

$$
f, g \notin \bigcup_{i<k \leq 5} S_{k} \quad \text { and } \quad g \notin \bigcup_{j<k \leq i} S_{k} .
$$

Indeed, if $f$ or $g$ belongs to $S_{k}$ for some $k>i$, then let $k$ be the maximum index such that $S_{k}$ contains either $f$ or $g$. Then by the observation above, there exists some $j \neq k$ such that one signature belongs to $S_{j} \backslash S_{k}$, and the other one belongs to $S_{k} \backslash S_{j}$. By the maximality of $k$, we have $k>j$. Since $k>i$ and No-Mixing theorem$(k, j)$ has already been proved, we have $\mathrm{Pl}-\# \mathrm{CSP}^{2}(f, g)$ is \#P-hard. Moreover, if $g \in \bigcup_{j<\ell \leq i} S_{\ell}$, then $g \in S_{\ell}$ for some $j<\ell<i$, as $g \notin S_{i}$. Then $f \in S_{i} \backslash$ $S_{\ell}$ since $\{f, g\} \nsubseteq S_{\ell}$, and also $g \in S_{\ell} \backslash S_{i}$. Hence $\mathrm{Pl}$ \#CSP${ }^{2}(f, g)$ is \#P-hard by No-Mixing- $(i, \ell)$ already proved.

We now proceed with this plan. We first prove a preliminary result, which allows us to construct signatures of arbitrarily high even arities from a given binary signature.

Lemma 14.1 For any binary signature $[a, b, c]$, any integer $k \geq 1$, and any signature set $\mathcal{F}$,

$$
\mathrm{Pl}-\# \operatorname{CSP}^{2}\left([a, b]^{\otimes 2 k}+[b, c]^{\otimes 2 k}, \mathcal{F}\right) \leq_{T} \operatorname{Pl}-\# \operatorname{CSP}^{2}([a, b, c], \mathcal{F}) .
$$

Proof We take $2 k$ copies of $[a, b, c]$ and connect one input of each $[a, b, c]$ to an edge of $=2 k$. The resulting signature is $[a, b]^{\otimes 2 k}+[b, c]^{\otimes 2 k}$, since $\left[\begin{array}{ll}a & b \\ b & c\end{array}\right]^{\otimes n}\left(\left[\begin{array}{l}1 \\ 0\end{array}\right]^{\otimes n}+\left[\begin{array}{l}0 \\ 1\end{array}\right]^{\otimes n}\right)=\left[\begin{array}{l}a \\ b\end{array}\right]^{\otimes n}+\left[\begin{array}{l}b \\ c\end{array}\right]^{\otimes n}$.

In the next lemma, we will prove that for any symmetric signature $f \in \mathscr{A} \backslash \mathscr{P}$ of even arity, we can construct an arity 4 signature $g \in \mathscr{A} \backslash \mathscr{P}$ in $\operatorname{Pl-\# CSP}{ }^{2}(\{f\} \cup \mathcal{F})$. Thus we can assume that we have an arity 4 signature $g \in \mathscr{A} \backslash \mathscr{P}$ in the proof of the No-Mixing lemma of $\mathscr{P}$ versus $\mathscr{A}$, namely No-Mixing- $(5,4)$. We can prove a similar result for $\mathscr{A}^{\dagger} \backslash \mathscr{P}$. This is for the proof of No-Mixing-(5, 3). 
Lemma 14.2 For any symmetric signature $f \in \mathscr{A} \backslash \mathscr{P}$ (respectively, $f \in \mathscr{A}^{\dagger} \backslash \mathscr{P}$ ) of even arity $2 n \geq 2$, there exists a symmetric signature $g \in \mathscr{A} \backslash \mathscr{P}$ (respectively, $\left.g \in \mathscr{A}^{\dagger} \backslash \mathscr{P}\right)$ of arity 4 , such that for any set $\mathcal{F}$,

$$
\mathrm{Pl}-\# \operatorname{CSP}^{2}(\{g\} \cup \mathcal{F}) \leq_{T} \operatorname{Pl}-\# \operatorname{CSP}^{2}(\{f\} \cup \mathcal{F}) .
$$

Proof If $f$ has arity $2 n=4$, then there is nothing to prove. Suppose $2 n \neq 4$. For $f \in$ $\mathscr{A}^{\dagger} \backslash \mathscr{P}$, if $2 n=2$, then $f=\left[1, \alpha,-\alpha^{2}\right]$ by Corollary 9.9. By Lemma 14.1, we have $g=[1, \alpha]^{\otimes 4}-[1,-\alpha]^{\otimes 4}$, since $\alpha^{4}=-1$. Clearly $g \in \mathscr{A}^{\dagger}$ and is non-degenerate. Note that $g$ satisfies a second-order recurrence relation of type $\left\langle-\alpha^{2}, 0,1\right\rangle$, since the eigenvalues of the recurrence are $\pm \alpha$ with trace 0 and product $-\alpha^{2}$. Thus $g \notin \mathscr{P}$ by Lemma 9.11. For $2 n \geq 6$, we have $f=[1, \alpha]^{\otimes 2 n}+i^{r}[1,-\alpha]^{\otimes 2 n}$ by definitions (see Fig. 24). Then by our calculus, we have $\partial^{n-2}(f)=\left(1+\alpha^{2}\right)^{n-2}\left\{[1, \alpha]^{\otimes 4}+\right.$ $\left.i^{r}[1,-\alpha]^{\otimes 4}\right\}$. Clearly it is in $\mathscr{A}^{\dagger}$ and is non-degenerate. It also has type $\left\langle-\alpha^{2}, 0,1\right\rangle$ and therefore it is not in $\mathscr{P}$.

For $f \in \mathscr{A} \backslash \mathscr{P}$, if $2 n=2$, then $f=\left[1, \rho,-\rho^{2}\right]$ by Corollary 9.9. By Lemma 14.1, we have $g=[1, \rho]^{\otimes 4}+[1,-\rho]^{\otimes 4}$, since $\rho^{4}=1$. Clearly $g \in \mathscr{A}$ and is non-degenerate. Note that $g$ has type $\left\langle-\rho^{2}, 0,1\right\rangle$, since the eigenvalues of its second-order recurrence relation are $\pm \rho$ with trace 0 and product $-\rho^{2}$. Thus $g \notin \mathscr{P}$ by Lemma 9.11 .

For $2 n \geq 6$, we have $f=[1, \rho]^{\otimes 2 n}+i^{r}[1,-\rho]^{\otimes 2 n}$ by definitions (see Fig. 24). If $2 n \equiv 0(\bmod 4)$, then $n$ is even, and we have $\partial_{=4}^{\frac{n-2}{2}}(f)=2^{\frac{n-2}{2}}\left\{[1, \rho]^{\otimes 4}+\right.$ $\left.i^{r}[1,-\rho]^{\otimes 4}\right\}$ that is in $\mathscr{A}$, and not in $\mathscr{P}$ by its type $\left\langle-\rho^{2}, 0,1\right\rangle$. For $2 n \equiv 2(\bmod 4)$, we have $h=\partial_{=4}^{\frac{n-1}{2}}(f)=2^{\frac{n-1}{2}}\left\{[1, \rho]^{\otimes 2}+i^{r}[1,-\rho]^{\otimes 2}\right\}$.

- If $r=2$, then we have $h=2^{\frac{n-1}{2}}[0,2 \rho, 0]$. Thus we have $[0,1,0]$ up to a nonzero scalar and $\partial_{[0,1,0]}^{n-2}(f)=(2 \rho)^{n-2}\left\{[1, \rho]^{\otimes 4}+i^{r}(-1)^{n-2}[1,-\rho]^{\otimes 4}\right\}$ that is in $\mathscr{A}$, and not in $\mathscr{P}$ by its type $\left\langle-\rho^{2}, 0,1\right\rangle$.

- If $r \neq 2$, then $h=2^{\frac{n-1}{2}}\left(1+i^{r}\right)\left[1, \frac{1-i^{r}}{1+i^{r}} \rho, \rho^{2}\right]$. Then we have $\partial_{\left[1, \frac{1-i^{r}}{1+i^{r}} \rho, \rho^{2}\right]}(=4)=$ $\left[1,0, \rho^{2}\right]$ on LHS and $\partial_{\left[1,0, \rho^{2}\right]}^{n-2}(f)=2^{n-2}\left\{[1, \rho]^{\otimes 4}+i^{r}[1,-\rho]^{\otimes 4}\right\}$ by $\rho^{4}=1$, that is in $\mathscr{A} \backslash \mathscr{P}$ by the same reason.

We note that the complication for the case $f \in \mathscr{A} \backslash \mathscr{P}$ is unavoidable since if $\rho= \pm i$, then $\partial(f)=0$, therefore we need to use $\partial_{=_{4}}(f)$.

\subsection{Mixing with $S_{5}=\mathscr{P}$}

In this subsection, we prove No-Mixing- $(5, j)$, for $1 \leq j \leq 4$, namely the NoMixing of one signature in $\mathscr{P}$ and another signature in a different tractable set. Thus we assume there is some $f \in S_{5}=\mathscr{P}$, and some $g \in S_{k}$ for some $1 \leq k \leq 4$, and for no $1 \leq k \leq 5,\{f, g\} \subset S_{k}$. Under this assumption we show that $\mathrm{Pl}_{-\# \mathrm{CSP}^{2}}(f, g)$ is \#P-hard. As explained earlier, for $j<k<5$, when we prove No-Mixing- $(5, j)$, we can make logical use of No-Mixing- $(5, k)$. 
Lemma 14.3 Let $\{f, g\} \subseteq \bigcup_{k=1}^{5} S_{k}$ and $\{f, g\} \nsubseteq S_{j}$ for every $1 \leq j \leq 5$. Assume that $f \in S_{5}=\mathscr{P}$, then $\mathrm{Pl}-\# \mathrm{CSP}^{2}(f, g)$ is \#P-hard.

Proof As explained earlier, since $f \in \mathscr{P}$, there exists some $1 \leq k \leq 4$, such that $g \in S_{k} \backslash \mathscr{P}$ and $f \in \mathscr{P} \backslash S_{k}$. Since $[0,1,0] \in \bigcap_{k=1}^{5} S_{k}$, we know that $f$ is not a multiple of $[0,1,0]$. Then by $f \in \mathscr{P}$ (see Fig. 24), we have $f=[a, b]^{\otimes 2 n}$ with $a$ and $b$ not both 0 (because $f$ is not identically 0 ), or $f=[1,0, \ldots, 0, x]$ with $x \neq 0$.

We first consider the case $f=[a, b]^{\otimes 2 n}$, with $(a, b) \neq(0,0)$. It has three subcases.

- If $a b \neq 0$ (i.e., $a$ and $b$ both nonzero) and $a^{2}+b^{2} \neq 0$, then we have $\partial^{n-1}(f)=$ $\left(a^{2}+b^{2}\right)^{n-1}[a, b]^{\otimes 2}$. We are done by Lemma 10.3 .

- If $a b \neq 0$ and $a^{2}+b^{2}=0$, then $f=[1, \pm i]^{\otimes 2 n}$ up to a nonzero scalar. Note that $f \in \mathscr{P} \cap \mathscr{A} \cap \widehat{\mathscr{M}^{\dagger}}$. Hence $g \in \mathscr{A}^{\dagger} \backslash\left(\mathscr{P} \cup \mathscr{A} \cup \widehat{\mathscr{M}^{\dagger}}\right)$ or $g \in \widehat{\mathscr{M} \backslash}\left(\mathscr{P} \cup \mathscr{A} \cup \widehat{\mathscr{M}}{ }^{\dagger}\right)$.

If $g \in \widehat{\mathscr{M}} \backslash\left(\mathscr{P} \cup \widetilde{\mathscr{A}} \cup \widehat{\mathscr{M}}^{\dagger}\right)$, then a fortiori, $g \in \widehat{\mathscr{M}} \backslash(\mathscr{P} \cup \mathscr{\mathscr { A }})$. Therefore we are done by Lemma 13.3.

The other case is $g \in \mathscr{A}^{\dagger} \backslash\left(\mathscr{P} \cup \mathscr{A} \cup \widehat{\mathscr{M}}^{\dagger}\right)$, then a fortiori, $g \in \mathscr{A}^{\dagger} \backslash \mathscr{P}$, and by Lemma 14.2, we have an arity 4 signature $g^{\prime} \in \mathscr{A}^{\dagger} \backslash \mathscr{P}$. By definition (see Fig. 24), $g^{\prime}=[1, \alpha]^{\otimes 4}+i^{r}[1,-\alpha]^{\otimes 4}$. For $r=2$, we have $\partial\left(g^{\prime}\right)=2 \alpha(1+$ $\left.\alpha^{2}\right)[0,1,0]$ and $\partial_{[0,1,0]}^{n-1}(f)=( \pm 2 i)^{n-1}[1, \pm i]^{\otimes 2}$. Then we are done by Lemma 10.3. For $r \neq 2$, we have on LHS

$$
\begin{aligned}
\partial_{g^{\prime}}(=6) & =\partial_{[1, \alpha]^{\otimes 4}}(=6)+i^{r} \partial_{[1,-\alpha]^{\otimes 4}}(=6)=\left[1,0, \alpha^{4}\right]+i^{r}\left[1,0,(-\alpha)^{4}\right] \\
& =\left(1+i^{r}\right)[1,0,-1]
\end{aligned}
$$

and $\partial_{[1,0,-1]}^{n-1}(f)=2^{n-1}[1, \pm i]^{\otimes 2}$. Then again we are done by Lemma 10.3.

- For $f=[1,0]^{\otimes 2 n}$ or $[0,1]^{\otimes 2 n}$, we have $\partial^{n-1}(f)=[1,0]^{\otimes 2}$ or $[0,1]^{\otimes 2}$. Note

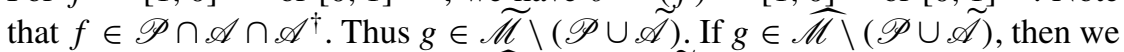
are done by Lemma 13.3. If $g \in \widehat{\mathscr{M}}^{\dagger} \backslash(\mathscr{P} \cup \mathscr{\mathscr { A }})$, then we are done by Lemma 13.7, where the binary signature is supplied by $\partial^{n-1}(f)=[1,0]^{\otimes 2}$ or $[0,1]^{\otimes 2}$.

The remaining case is $f=[1,0, \ldots, 0, x]$ with $x \neq 0$. We have $\partial^{n-1}(f)=[1,0, x]$. Suppose $g \in \mathscr{A}$. As $f \in \mathscr{P}$, we have $g \notin \mathscr{P}$. Then we have an arity 4 signature $g^{\prime} \in \mathscr{A} \backslash \mathscr{P}$ by Lemma 14.2. Moreover, by definition (see Fig. 24), we have $g^{\prime}=$ $[1, \gamma]^{\otimes 4}+i^{r}[1,-\gamma]^{\otimes 4}$ where $\gamma^{8}=1$. Depending on whether $g \in \mathscr{A}$ or $\mathscr{A}^{\dagger}$, we have either $f \in \mathscr{P} \backslash \mathscr{A}$, or $f \in \mathscr{P} \backslash \mathscr{A}^{\dagger}$. Then we claim that $x^{4} \neq 1$. Note that $f$ has even arity $2 n$. If $x^{4}=1$, then $f=[1,0, \ldots, 0, x] \in \mathscr{A}$ as well as $\left[\begin{array}{ll}1 & 0 \\ 0 & \alpha\end{array}\right]^{\otimes 2 n} f=\left[1,0, \ldots, 0, x i^{n}\right] \in \mathscr{A}$ thus $f \in \mathscr{A}^{\dagger}$. This is a contradiction. Thus we have $x^{4} \neq 0,1$. Let $\widehat{g^{\prime}}=\left[1, x^{-\frac{1}{2}} \gamma\right]^{\otimes 4}+i^{r}\left[1,-x^{-\frac{1}{2}} \gamma\right]^{\otimes 4}$. Then by Lemma 11.8 , $\mathrm{Pl}-\# \mathrm{CSP}^{2}\left(\widehat{g^{\prime}}\right) \leq \mathrm{Pl}-\# \mathrm{CSP}^{2}(f, g)$. Note that ${\widehat{g^{\prime}}}^{\prime}$ has type $\left\langle-x^{-1} \gamma^{2}, 0,1\right\rangle$ by calculating the trace and product of the eigenvalues of the second order recurrence relation. Note that $\left(-x^{-1} \gamma^{2}\right)^{4}=x^{-4} \neq 0,1$. Thus $\widehat{g^{\prime}} \notin \mathscr{P} \cup \widetilde{\mathscr{A}} \cup \widetilde{\mathscr{M}}$ by Lemma 9.11. This implies that $\mathrm{Pl}-\# \operatorname{CSP}^{2}\left(\widehat{g^{\prime}}\right)$ is \#P-hard by Theorem 12.5. So $\mathrm{Pl}$-\#CSP${ }^{2}(f, g)$ is \#P-hard.

Now we may assume that $g \notin \tilde{\mathscr{A}}$. Thus $g \in \widetilde{\mathscr{M} \backslash} \backslash(\mathscr{P} \cup \widetilde{\mathscr{A}})$. If $g \in \widehat{\mathscr{M} \backslash}(\mathscr{P} \cup \tilde{\mathscr{A}})$, then we are done by Lemma 13.3. If $g \in \widehat{\mathscr{M}}^{\dagger} \backslash(\mathscr{P} \cup \mathscr{\mathscr { A }})$, then $f \in \mathscr{P} \backslash \widehat{\mathscr{M}} \widehat{C}^{\dagger}$. In this case we claim that $x \neq 1$. Suppose for a contradiction that $x=1$, then we show that $f \in \widehat{\mathscr{M}^{\dagger}}$. Notice that $f=[1,0, \ldots, 0,1]=(=2 n)$ and $\widehat{\mathscr{M}}^{\dagger}=Z \mathscr{M}$, where 
$Z=\left[\begin{array}{ll}1 & 1 \\ i & -i\end{array}\right]$. Crucially recall that $f$ has even arity. Then, up to a nonzero scalar, $\left(Z^{-1}\right)^{\otimes 2 n} f=[1,0,1, \ldots, 0,1] \in \mathscr{M}$ of arity $2 n$ (if $n$ is even) or $\left(Z^{-1}\right)^{\otimes 2 n} f=$ $[0,1,0, \ldots, 1,0] \in \mathscr{M}$ of arity $2 n$ (if $n$ is odd). Hence $x \neq 1$. Then we are done by Lemma 13.7, with $g \in \widehat{\mathscr{M}} \backslash(\mathscr{P} \cup \widetilde{\mathscr{A}})$, and the help of $\partial^{n-1}(f)=[1,0, x]$.

\subsection{Mixing with $S_{4}=\mathscr{A}$}

In this subsection, we prove the No-Mixing lemma of $\mathscr{A}$ with other tractable sets. Because we have already proved Lemma 14.3, the No-Mixing lemma for $S_{5}=\mathscr{P}$, we only need to consider No-Mixing- $(4, j)$ of $S_{4}=\mathscr{A}$ with $S_{j}$ for $1 \leq j \leq 3$.

There is a particular case involving $\mathscr{A}$ and $\mathscr{A}^{\dagger}$ that requires some special care. This is when two signatures $f \in \mathscr{A}$ and $g \in \mathscr{A}^{\dagger}$ both satisfy the parity constraints. We deal with this case first. Furthermore, by Lemma 14.2, for two signatures $f \in$ $\mathscr{A} \backslash \mathscr{P}$ and $g \in \mathscr{A}^{\dagger} \backslash \mathscr{P}$ we may assume the signatures $f$ and $g$ have arity 4. Hence the next lemma considers signatures $f$ and $g$ of arity 4 .

Lemma 14.4 Let $f=[1, \rho]^{\otimes 4} \pm[1,-\rho]^{\otimes 4} \in \mathscr{A}$ and $g=[1, \alpha]^{\otimes 4} \pm[1,-\alpha]^{\otimes 4} \in$ $\mathscr{A}^{\dagger}$. Then $\mathrm{Pl}$-\#CSP${ }^{2}(f, g)$ is \#P-hard.

Proof There are four cases depending on the combination of the two \pm signs. Suppose $f=[1, \rho]^{\otimes 4}+[1,-\rho]^{\otimes 4}$ and $g=[1, \alpha]^{\otimes 4}+[1,-\alpha]^{\otimes 4}$. Consider the gadget in Fig. 34a. We assign $g$ to the circle vertex and $f$ to the triangle vertex. Since both $f=2\left[1,0, \rho^{2}, 0,1\right]$ and $g=2\left[1,0, \alpha^{2}, 0,-1\right]$ have even parity, the signature of this gadget also has even parity. It is also clearly a redundant signature by design. Hence there are only five signature entries we need to compute. E.g., the entry of Hamming weight 0 is $g_{0} f_{0}+g_{2} f_{2}=4\left(1+\alpha^{2} \rho^{2}\right)$. Up to a factor of 4 , the signature of this gadget has signature matrix

$$
\begin{aligned}
& {\left[\begin{array}{cccc}
\alpha^{2} \rho^{2}+1 & 0 & 0 & \alpha^{2}+\rho^{2} \\
0 & 2 \alpha^{2} \rho^{2} & 2 \alpha^{2} \rho^{2} & 0 \\
0 & 2 \alpha^{2} \rho^{2} & 2 \alpha^{2} \rho^{2} & 0 \\
\alpha^{2}-\rho^{2} & 0 & 0 & \alpha^{2} \rho^{2}-1
\end{array}\right] \text {, which becomes }} \\
& {\left[\begin{array}{cccc}
\alpha^{2} \rho^{2}+1 & 0 & 0 & 2 \alpha^{2} \rho^{2} \\
0 & \alpha^{2}-\rho^{2} & 2 \alpha^{2} \rho^{2} & 0 \\
0 & 2 \alpha^{2} \rho^{2} & \alpha^{2}+\rho^{2} & 0 \\
2 \alpha^{2} \rho^{2} & 0 & 0 & \alpha^{2} \rho^{2}-1
\end{array}\right]}
\end{aligned}
$$

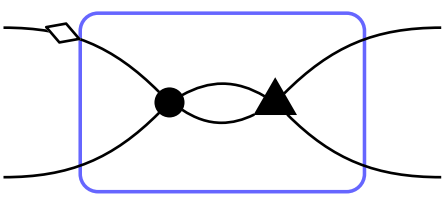

(a)

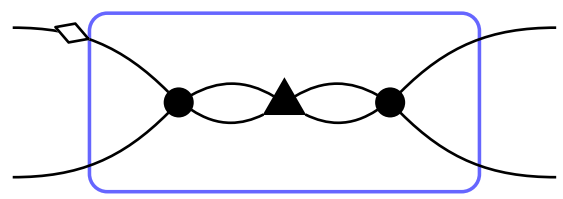

(b)

Fig. 34 Two gadgets used in the proof of Lemma 14.4 
after a $90^{\circ}$ counterclockwise rotation of the gadget. (See Fig. 2 in Part I for an illustration of the rotation operation.) Taking the four corner entries, we define the binary signature $h=\left[\alpha^{2} \rho^{2}+1,2 \alpha^{2} \rho^{2}, \alpha^{2} \rho^{2}-1\right]$. By domain pairing, $\operatorname{Pl}$ \#CSP $(h) \leq_{T}$ $\mathrm{Pl}-\# \mathrm{CSP}^{2}(f, g)$. (Domain pairing is the following reduction: In an instance of $\mathrm{Pl}$-\#CSP $(h)$ replace every occurrence of $h$ by a copy of the $90^{\circ}$ counterclockwise rotated gadget, and replace both edges of $h$ by two parallel edges each, and replace every $(=k)$ in the $\mathrm{Pl}$-\#CSP $(h)$ instance by $(=2 k)$ in $\operatorname{Pl}_{-} \# \operatorname{CSP}^{2}(f, g)$. Note that the rotation is necessary to create a symmetric binary signature $h$ in the paired domain.)

Note that $\alpha^{2}= \pm i$ and $\rho^{2}= \pm 1$, so $\alpha^{2} \rho^{2} \pm 1$ has norm $\sqrt{2}$, while $2 \alpha^{2} \rho^{2}$ has norm 2. Also $\alpha^{2} \rho^{2}+1 \neq \alpha^{2} \rho^{2}-1$. Hence $h \notin \mathscr{P} \cup \mathscr{A}$ by Corollary 9.9 and also $h \notin \widehat{\mathscr{M}}$ by Lemma 9.8. Thus Pl-\#CSP $(h)$ is \#P-hard by Theorem 9.22. $\operatorname{So~} P l-\# C S P^{2}(f, g)$ is \#P-hard.

Suppose $f=[1, \rho]^{\otimes 4}-[1,-\rho]^{\otimes 4}$ and $g=[1, \alpha]^{\otimes 4}-[1,-\alpha]^{\otimes 4}$. Consider the same construction. Up to a nonzero factor of $4 \alpha \rho$, the signature of this gadget has the signature matrix

$$
\begin{aligned}
& {\left[\begin{array}{cccc}
2 & 0 & 0 & 2 \rho^{2} \\
0 & 1+\alpha^{2} \rho^{2} & 1+\alpha^{2} \rho^{2} & 0 \\
0 & 1+\alpha^{2} \rho^{2} & 1+\alpha^{2} \rho^{2} & 0 \\
2 \alpha^{2} & 0 & 0 & 2 \alpha^{2} \rho^{2}
\end{array}\right] \text {, which becomes }} \\
& {\left[\begin{array}{cccc}
2 & 0 & 0 & 1+\alpha^{2} \rho^{2} \\
0 & 2 \alpha^{2} & 1+\alpha^{2} \rho^{2} & 0 \\
0 & 1+\alpha^{2} \rho^{2} & 2 \rho^{2} & 0 \\
1+\alpha^{2} \rho^{2} & 0 & 0 & 2 \alpha^{2} \rho^{2}
\end{array}\right]}
\end{aligned}
$$

after a $90^{\circ}$ counterclockwise rotation of the gadget. Let $h=\left[2,1+\alpha^{2} \rho^{2}, 2 \alpha^{2} \rho^{2}\right]$. By domain pairing, we have $\mathrm{Pl}-\# \operatorname{CSP}(h) \leq_{T} \operatorname{Pl}^{-\# C_{C S P}}(f, g)$. Note that $1+\alpha^{2} \rho^{2}=1 \pm i$ has norm $\sqrt{2}$ while $2 \alpha^{2} \rho^{2} \neq 2$ but has norm 2 . Hence $h \notin \mathscr{P} \cup \mathscr{A} \cup \widehat{\mathscr{M}}$ by Corollary 9.9 and Lemma 9.8. Thus we are done by Theorem 9.22.

Suppose $f=[1, \rho]^{\otimes 4}-[1,-\rho]^{\otimes 4}$ and $g=[1, \alpha]^{\otimes 4}+[1,-\alpha]^{\otimes 4}$. Consider the gadget in Fig. 34b. We assign $f$ to the circle vertices and $g$ to the triangle vertex. Up to a nonzero factor of $16 \alpha^{2} \rho^{2}$, the signature of this gadget has the signature matrix

$$
\left[\begin{array}{cccc}
2 & 0 & 0 & 2 \rho^{2} \\
0 & \rho^{2} & \rho^{2} & 0 \\
0 & \rho^{2} & \rho^{2} & 0 \\
2 \rho^{2} & 0 & 0 & 2
\end{array}\right], \quad \text { which becomes } \quad\left[\begin{array}{cccc}
2 & 0 & 0 & \rho^{2} \\
0 & 2 \rho^{2} & \rho^{2} & 0 \\
0 & \rho^{2} & 2 \rho^{2} & 0 \\
\rho^{2} & 0 & 0 & 2
\end{array}\right]
$$

after a $90^{\circ}$ rotation of the gadget. Let $h=\left[2, \rho^{2}, 2\right]$. We also have $g^{\times}=$ $2\left[1, \alpha^{2}\right]^{\otimes 2}$ by domain pairing with $g$ (see Lemma 9.19). Then $\operatorname{Pl}$-\#CSP $\left(g^{\times}, h\right) \leq_{T}$ 
Pl-\#CSP ${ }^{2}(f, g)$. Note that $\left|\rho^{2}\right|=1 \neq 2$, so by Lemma 9.8 and Corollary 9.9, $h \in \widehat{\mathscr{M}} \backslash(\mathscr{P} \cup \mathscr{A})$. Also by Lemma 9.8 and $\left(\alpha^{2}\right)^{2}=-1 \neq 1$ we have $g^{\times} \notin \widehat{\mathscr{M}}$. Thus we are done by Theorem 9.22. Note that in this case, the rotation is necessary to create a non-degenerate binary signature $h$ in the paired domain.

Finally, suppose $f=[1, \rho]^{\otimes 4}+[1,-\rho]^{\otimes 4}$ and $g=[1, \alpha]^{\otimes 4}-[1,-\alpha]^{\otimes 4}$. Consider the gadget in Fig. 34b. We assign $g$ to the circle vertices and $f$ to the triangle vertex. Up to a nonzero factor of $16 \alpha^{2} \rho^{2}$, the signature of this gadget has the signature matrix

$$
\left[\begin{array}{cccc}
2 & 0 & 0 & 2 \alpha^{2} \\
0 & \alpha^{2} & \alpha^{2} & 0 \\
0 & \alpha^{2} & \alpha^{2} & 0 \\
2 \alpha^{2} & 0 & 0 & -2
\end{array}\right], \quad \text { which becomes } \quad\left[\begin{array}{cccc}
2 & 0 & 0 & \alpha^{2} \\
0 & \alpha^{2} & 2 \alpha^{2} & 0 \\
0 & 2 \alpha^{2} & \alpha^{2} & 0 \\
\alpha^{2} & 0 & 0 & -2
\end{array}\right]
$$

after a $90^{\circ}$ rotation of the gadget. Let $h=\left[2, \alpha^{2},-2\right]$, then $\mathrm{Pl}$-\#CSP $(h) \leq_{T}$ $\mathrm{Pl}-\# \mathrm{CSP}^{2}(f, g)$ by domain pairing. Since $\left|\alpha^{2}\right|=1 \neq 2$, we have $h \notin \mathscr{P} \cup \mathscr{A}$ by Corollary 9.9 and also $h \notin \widehat{\mathscr{M}}$ by Lemma 9.8. Thus we are done by Theorem 9.22. Note that in this case, the rotation is also necessary to create a non-degenerate binary signature $h$ in the paired domain.

Remark 8 The use of a more complicated construction in the third case is necessary. Notice that $g=[1, \alpha]^{\otimes 4}+[1,-\alpha]^{\otimes 4}=2\left[1,0, \alpha^{2}, 0,-1\right]$ has an even parity, while $f=[1, \rho]^{\otimes 4}-[1,-\rho]^{\otimes 4}=2 \rho\left[0,1,0, \rho^{2}, 0\right]$ has an odd parity. Then in any construction of a signature using $f$ and $g$, if the number of occurrences $N_{f}$ of $f$ is odd (resp. even), then the resulting signature also has an odd (resp. even) parity. To see this, let $H$ be an arbitrary $\{f, g\}$-gate with $N_{f}$ occurrences of $f$. Suppose $\sigma$ is a $\{0,1\}$-assignment to all the edges of $H$, including internal and external edges, that has a nonzero evaluation on $H$. Then each copy of $f$ has an odd number of incident edges assigned to 1 . Summing these numbers (mod 2) over all copies of $f$ we get a number $\equiv N_{f}(\bmod 2)$, since each of these numbers is $\equiv 1(\bmod 2)$. Similarly each copy of $g$ has an even number of incident edges assigned to 1. Summing these numbers $(\bmod 2)$ over all copies of $g$ we get a number $\equiv 0(\bmod 2)$. On the other hand, if we add these two sums together we get $2 X+Y$ where $X$ is the number of internal edges and $Y$ is the number of external edges assigned to 1 by $\sigma$. This is because each internal edge assigned to 1 appears exactly twice in the sum. Hence this number is $\equiv Y(\bmod 2)$. We conclude that $N_{f} \equiv Y(\bmod 2)$, the Hamming weight of $\sigma$ on the external edges.

If $N_{f}$ is odd, from any constructed signature of arity 4 , by rotation and domain pairing we can only get the identically zero binary signature. Thus we must use $f$ an even number of times. Using $g$ alone will not get out of $\mathscr{A}^{\dagger}$, which is a tractable set. Thus we must use $f$ at least twice. Also using $g$ alone will not get out of $\mathscr{A}$, another tractable set. Therefore we must use $g$ at least once. Therefore the construction we give is the simplest possible.

The same consideration applies for the construction in the fourth case. 
The next Lemma deals with the situation when we have a binary signature in $\mathscr{A} \backslash \mathscr{P}$ and an arity 4 signature in $\mathscr{A}^{\dagger} \backslash \mathscr{P}$.

Lemma 14.5 Let $f=\left[1, \rho,-\rho^{2}\right]$ and $g=[1, \alpha]^{\otimes 4}+i^{r}[1,-\alpha]^{\otimes 4}$. Then $\mathrm{Pl}-\# \mathrm{CSP}^{2}(f, g)$ is \#P-hard.

Proof By our calculus, we have $\partial_{\left[1, \rho,-\rho^{2}\right]}(g)=\lambda[1, \alpha]^{\otimes 2}+i^{r} \mu[1,-\alpha]^{\otimes 2}$, where $\lambda=1-\rho^{2} \alpha^{2}+2 \rho \alpha$ and $\mu=1-\rho^{2} \alpha^{2}-2 \rho \alpha$. Note that $1-\rho^{2} \alpha^{2}=$ $1 \pm i$ has norm $\sqrt{2}$ and $|2 \rho \alpha|=2$, we have $\lambda \neq 0$. Let $x=i^{r} \mu / \lambda$, then $\partial_{\left[1, \rho,-\rho^{2}\right]}(g)=\lambda(1+x)\left[1, \frac{1-x}{1+x} \alpha, \alpha^{2}\right]$. By norm, $\left(1-\rho^{2} \alpha^{2}\right)^{4} \neq(2 \rho \alpha)^{4}$ and $\left(1-\rho^{2} \alpha^{2}\right)(2 \rho \alpha) \neq 0$, we have $x^{4} \neq 0,1$ by Lemma 9.3. By Lemma 9.3 again, we have $\left(\frac{1-x}{1+x}\right)^{4} \neq 0,1$. Thus $\left[1, \frac{1-x}{1+x} \alpha, \alpha^{2}\right] \notin \mathscr{P} \cup \widetilde{\mathscr{A}} \cup \widetilde{\mathscr{M}}$ by Corollary 9.9. This implies that $\mathrm{Pl}-\# \operatorname{CSP}^{2}\left(\left[1, \frac{1-x}{1+x} \alpha, \alpha^{2}\right]\right)$ is \#P-hard by Theorem 9.21'. Thus $\mathrm{Pl}-\# \mathrm{CSP}^{2}(f, g)$ is \#P-hard.

The next lemma is the No-Mixing lemma of $\mathscr{A}$ with the other tractable sets, namely the statements No-Mixing- $(4, j)$ for $1 \leq j \leq 3$. Having already proved Lemma 14.3, we can assume that both $f$ and $g$ are not in $S_{5}=\mathscr{P}$.

Lemma 14.6 Let $\{f, g\} \subseteq\left(\bigcup_{k=1}^{4} S_{k}\right) \backslash S_{5}$ and $\{f, g\} \nsubseteq S_{j}$ for every $1 \leq j \leq 4$. Assume that $f \in S_{4}=\mathscr{A}$, then $\mathrm{Pl}-\# \operatorname{CSP}^{2}(f, g)$ is \#P-hard.

Proof By $f \in \mathscr{A}$, we have $g \notin \mathscr{A}$. Thus, $g \in\left(\mathscr{A}^{\dagger} \cup \widehat{\mathscr{M} \cup} \widehat{\mathscr{M}} \widehat{\mathscr{M}}^{\dagger}\right) \backslash(\mathscr{P} \cup \mathscr{A})$.

1. Suppose $g \in \mathscr{A}^{\dagger} \backslash(\mathscr{P} \cup \mathscr{A})$. Then a fortiori, $g \in \mathscr{A}^{\dagger} \backslash \mathscr{P}$. As $f \in \mathscr{A} \backslash \mathscr{P}$, by Lemma 14.2, we have some $f^{\prime} \in \mathscr{A} \backslash \mathscr{P}$ and $g^{\prime} \in \mathscr{A}^{\dagger} \backslash \mathscr{P}$, both of arity 4. Without loss of generality, we will assume the given $f$ and $g$ are of arity 4. By definition (see Fig. 24), we can assume that

$f=[1, \rho]^{\otimes 4}+i^{r}[1,-\rho]^{\otimes 4}$ and $g=[1, \alpha]^{\otimes 4}+i^{s}[1,-\alpha]^{\otimes 4}$ where $r, s=0,1,2,3$.

- If both $r, s \equiv 0(\bmod 2)$, then $f=[1, \rho]^{\otimes 4} \pm[1,-\rho]^{\otimes 4}$ and $g=[1, \alpha]^{\otimes 4} \pm$ $[1,-\alpha]^{\otimes 4}$. This is the case where both $f$ and $g$ satisfy the parity constraints, and it is proved in Lemma 14.4.

- If $r \equiv 1(\bmod 2)$ then $f=[1, \rho]^{\otimes 4} \pm i[1,-\rho]^{\otimes 4}$. For $\rho^{2}=1$, by our calculus we have

$\partial(f)=2\left\{[1, \rho]^{\otimes 2} \pm i[1,-\rho]^{\otimes 2}\right\}=2(1 \pm i)\left[1, \mp i \rho, \rho^{2}\right]=2(1 \pm i)\left[1, \rho^{\prime},-\rho^{\prime 2}\right]$,

where $\rho^{\prime}=\mp i \rho$, and $\rho^{\prime 4}=1$. Thus Pl-\#CSP$\left(\left[1, \rho^{\prime},-\rho^{\prime 2}\right], g\right)$ is \#P-hard by Lemma 14.5. So Pl-\#CSP${ }^{2}(f, g)$ is \#P-hard.

For $\rho^{2}=-1$, we cannot use $[1,0,1]$ to reduce the arity of $f$, because $\partial(f)=0$ in this case. Instead we construct a suitable binary signature from $g$. If $s \neq 2$, then we have $g_{0}=1+i^{s} \neq 0$ and $g_{4}=\alpha^{4}+i^{s}(-\alpha)^{4}=$ $-\left(1+i^{s}\right)=-g_{0}$, and therefore $\partial_{g}\left(={ }_{6}\right)=g_{0}[1,0,-1]$ on the LHS. Then we have $\partial_{[1,0,-1]}(f)=2\left\{[1, \rho]^{\otimes 2} \pm i[1,-\rho]^{\otimes 2}\right\}=2(1 \pm i)\left[1, \mp i \rho, \rho^{2}\right]=$ $2(1 \pm i)\left[1, \rho^{\prime},-\rho^{\prime 2}\right]$, where $\rho^{\prime}=\mp i \rho$ and $\rho^{\prime 4}=1$. Then we are done 
by Lemma 14.5. If $s=2$, then $\partial(g)=\left(1+\alpha^{2}\right)\left\{[1, \alpha]^{\otimes 2}-[1,-\alpha]^{\otimes 2}\right\}$, a nonzero multiple of $[0,1,0]$. Thus we have $\partial_{[0,1,0]}(f)=2 \rho\left\{[1, \rho]^{\otimes 2} \mp\right.$ $\left.i[1,-\rho]^{\otimes 2}\right\}=2 \rho(1 \mp i)\left[1, \pm i \rho, \rho^{2}\right]=2 \rho(1 \mp i)\left[1, \rho^{\prime},-\rho^{\prime 2}\right]$, where $\rho^{\prime}=$ $\pm i \rho$ and $\rho^{\prime 4}=1$. Then we are done by Lemma 14.5 again.

- If $r \equiv 0(\bmod 2)$ and $s \equiv 1(\bmod 2)$, i.e., $f=[1, \rho]^{\otimes 4} \pm[1,-\rho]^{\otimes 4}$ and $g=[1, \alpha]^{\otimes 4} \pm i[1,-\alpha]^{\otimes 4}$, then we will construct a binary signature $h=[1, b, \pm 1]$. Note that $h \in \widetilde{\mathscr{M}}$ by Lemma 9.8. Furthermore, we will ensure that $b^{4} \neq 0,1$, thus $h \notin \mathscr{P} \cup \mathscr{A}$ by Corollary 9.9. Then we are done by Lemma 13.3 and Lemma 13.7.

We have $\partial(g)=\left(1+\alpha^{2}\right)\left\{[1, \alpha]^{\otimes 2} \pm i[1,-\alpha]^{\otimes 2}\right\}=\left(1+\alpha^{2}\right)(1 \pm$ $i)\left[1, \mp i \alpha, \alpha^{2}\right]$, a nonzero multiple of $\left[1, \alpha^{\prime},-\alpha^{\prime 2}\right]$, where $\alpha^{\prime}=\mp i \alpha$ and $\alpha^{\prime 4}=-1$. Moreover, we have $h=\partial_{\left[1, \alpha^{\prime},-\alpha^{\prime 2}\right]}(f)=\lambda[1, \rho]^{\otimes 2} \pm$ $\mu[1,-\rho]^{\otimes 2}$, where $\lambda=1-\rho^{2} \alpha^{\prime 2}+2 \rho \alpha^{\prime}$ and $\mu=1-\rho^{2} \alpha^{\prime 2}-2 \rho \alpha^{\prime}$. Then $h=\lambda(1 \pm x)\left[1, a \rho, \rho^{2}\right]$, where $x=\frac{\mu}{\lambda}$ and $a=\frac{1 \mp x}{1 \pm x}$. Note that $1-\rho^{2} \alpha^{\prime 2}=1 \pm i$ has norm $\sqrt{2}$ and $\left|2 \rho \alpha^{\prime}\right|=2$, thus $\lambda \neq 0$ and $\left(1-\rho^{2} \alpha^{\prime 2}\right)^{4} \neq\left(2 \rho \alpha^{\prime}\right)^{4}$ by norm. Denote by $u=1-\rho^{2} \alpha^{\prime 2}$ and $v=-2 \rho \alpha^{\prime}$. Then $x=\frac{u+v}{u-v}$. By Lemma 9.3, $x^{4} \neq 0,1$. Then by Lemma 9.3 again, $a^{4} \neq 0,1$, and so $(a \rho)^{4}=a^{4} \neq 0,1$ as well. As $\lambda \neq 0,1 \pm x \neq 0, \rho^{2}= \pm 1$, we have a nonzero multiple of $[1, a \rho, \pm 1]$, our desired binary signature, and we are done by Lemma 13.3 and Lemma 13.7.

In the following we may assume $g \notin \mathscr{A}^{\dagger}$.

2. Suppose $g \in \widehat{\mathscr{M}} \backslash(\mathscr{P} \cup \mathscr{A})$, then $g \in \widehat{\mathscr{M} \backslash}(\mathscr{P} \cup \widetilde{\mathscr{A}})$. We also have $f \notin \widehat{\mathscr{M}}$, lest $\{f, g\} \subseteq \widehat{\mathscr{M}}$, and we are done by Lemma 13.3.

3. Suppose $g \in \widehat{\mathscr{M}} \backslash(\mathscr{P} \cup \mathscr{A})$, then $g \in \widehat{\mathscr{M}} \backslash \backslash(\mathscr{P} \cup \tilde{\mathscr{A}})$. Now $f \in \mathscr{A} \backslash\left(\mathscr{P} \cup \widehat{\mathscr{M}}^{\dagger}\right)$. Note that $[1,1]^{\otimes 2 n} \pm[1,-1]^{\otimes 2 n} \in \widehat{\mathscr{M}}^{\dagger}$. This can be verified as follows: Let $Z=\left[\begin{array}{ll}1 & 1 \\ i & -i\end{array}\right]$, then $\widehat{\mathscr{M}} \dagger=Z \mathscr{M}$, and $Z^{-1}=\frac{1}{2}\left[\begin{array}{ll}1 & -i \\ 1 & i\end{array}\right]$. We first verify that $[1,0]^{\otimes 2 n} \pm[0,1]^{\otimes 2 n} \in \widehat{\mathscr{M}^{\dagger}}=Z \widehat{\mathscr{M}}$, by

$$
\begin{aligned}
{\left[\begin{array}{ll}
1 & -i \\
1 & i
\end{array}\right]^{\otimes 2 n}\left\{\left[\begin{array}{l}
1 \\
0
\end{array}\right]^{\otimes 2 n} \pm\left[\begin{array}{l}
0 \\
1
\end{array}\right]^{\otimes 2 n}\right\} } & =\left[\begin{array}{l}
1 \\
1
\end{array}\right]^{\otimes 2 n} \pm(-i)^{2 n}\left[\begin{array}{l}
1 \\
-1
\end{array}\right]^{\otimes 2 n} \\
& =\left[\begin{array}{l}
1 \\
1
\end{array}\right]^{\otimes 2 n} \pm(-1)^{n}\left[\begin{array}{l}
1 \\
-1
\end{array}\right]^{\otimes 2 n} \in \mathscr{M}
\end{aligned}
$$

Then notice that

$$
\left[\begin{array}{l}
1 \\
1
\end{array}\right]^{\otimes 2 n} \pm\left[\begin{array}{l}
1 \\
-1
\end{array}\right]^{\otimes 2 n}=\left[\begin{array}{cc}
1 & 1 \\
1 & -1
\end{array}\right]^{\otimes 2 n}\left\{\left[\begin{array}{l}
1 \\
0
\end{array}\right]^{\otimes 2 n} \pm\left[\begin{array}{l}
0 \\
1
\end{array}\right]^{\otimes 2 n}\right\} \in\left[\begin{array}{cc}
1 & 1 \\
1 & -1
\end{array}\right] \widehat{\mathscr{M}^{\dagger}}
$$

However

$$
\left[\begin{array}{cc}
1 & 1 \\
1 & -1
\end{array}\right]\left[\begin{array}{cc}
1 & 1 \\
i & -i
\end{array}\right]=\left[\begin{array}{cc}
1+i & 1-i \\
1-i & 1+i
\end{array}\right]=\left[\begin{array}{cc}
1 & 1 \\
i & -i
\end{array}\right]\left[\begin{array}{cc}
0 & 1-i \\
1+i & 0
\end{array}\right]
$$


and $\left[\begin{array}{cc}0 & 1-i \\ 1+i & 0\end{array}\right] \mathscr{M}=\mathscr{M}$, therefore $\left[\begin{array}{cc}1 & 1 \\ 1 & -1\end{array}\right] \widehat{\mathscr{M}}=\left[\begin{array}{cc}1 & 1 \\ 1 & -1\end{array}\right] Z \mathscr{M}=$ $Z\left[\begin{array}{cc}0 & 1-i \\ 1+i & 0\end{array}\right] \mathscr{M}=Z \mathscr{M}=\widehat{\mathscr{M}}$. (Also see Fig. 25).

Now, since $[1,1]^{\otimes 2 n} \pm[1,-1]^{\otimes 2 n} \in \widehat{\mathscr{M}}^{\dagger}$ and $f \notin \widehat{\mathscr{M}}^{\dagger}, f$ cannot take the form $[1,1]^{\otimes 2 n} \pm[1,-1]^{\otimes 2 n}$. Then by definition (see Fig. 24) $f$ takes the form $\left[1, \rho,-\rho^{2}\right]$, or $[1,1]^{\otimes 2 n} \pm i[1,-1]^{\otimes 2 n}$, or $[1, i]^{\otimes 2 n}+i^{r}[1,-i]^{\otimes 2 n}$, where $2 n \geq 4$.

The following three cases are immediately done by Lemma 13.7:

- $f=\left[1, \rho,-\rho^{2}\right]$.

- $f=[1,1]^{\otimes 2 n} \pm i[1,-1]^{\otimes 2 n}$ with $2 n \geq 4$, then we have $\partial^{n-1}(f)=2^{n-1}[1 \pm$ $i, 1 \mp i, 1 \pm i]$ which is not $\lambda[1,0,1]$.

- If $f=[1, i]^{\otimes 2 n}+i^{r}[1,-i]^{\otimes 2 n}$ with $2 n \equiv 2(\bmod 4)$, then we have $\partial_{=4}^{\frac{n-1}{2}}(f)=2^{\frac{n-1}{2}}\left[1+i^{r},\left(1-i^{r}\right) i,-\left(1+i^{r}\right)\right]$ which is not $\lambda[1,0,1]$, no matter what value $r$ takes.

The remaining case is that $f=[1, i]^{\otimes 2 n}+i^{r}[1,-i]^{\otimes 2 n}$ with $2 n \equiv 0$ $(\bmod 4)$. In this case, we have

$$
\partial_{=4}^{\frac{n-2}{2}}(f)=2^{\frac{n-2}{2}}\left\{[1, i]^{\otimes 4}+i^{r}[1,-i]^{\otimes 4}\right\} .
$$

We will denote by $f^{\prime}=[1, i]^{\otimes 4}+i^{r}[1,-i]^{\otimes 4}$. If $g$ has arity 2 , then up to a nonzero scalar, $g=[1, b,-1]$ with $b^{4} \neq 0,1$ by Lemma 9.14 , and we are done by Lemma 13.7. In the following, assume that $g$ has arity $2 m \geq 4$. By Lemma 9.14, either $g=[s, t i]^{\otimes 2 m} \pm[t, s i]^{\otimes 2 m}$ with $s^{4} \neq t^{4}$ and $s t \neq 0$, or $g$ has arity $2 m$ and $g_{k}=(\epsilon i)^{k}(2 m-2 k)$.

- If $g$ has arity $2 m \geq 4$ and $g_{k}=(\epsilon i)^{k}(2 m-2 k)$ up to a nonzero scalar, then let $\hat{g}=\left(Z^{-1}\right)^{\otimes 2 m} g$, where $Z=\left[\begin{array}{ll}1 & 1 \\ i & -i\end{array}\right]$. Then $\hat{g}=[0,1,0, \ldots, 0]$ or $\hat{g}=[0, \ldots, 0,1,0]$ of arity $2 m$. By Corollary 10.5 , we have

$$
\mathrm{Pl}-\# \mathrm{CSP}^{2}(\hat{g}) \leq \mathrm{Pl} \# \operatorname{CSP}^{2}\left(f^{\prime}, g\right) \text {. }
$$

Let $\hat{g}^{\prime}=\partial^{m-2}(\hat{g})=[0,1,0,0,0]$ or $[0,0,0,1,0]$. Clearly $\hat{g}^{\prime}$ is nondegenerate. It also has a second order recurrence of type $\langle 0,0,1\rangle$ or $\langle 1,0,0\rangle$. By Lemma 9.11, $\hat{g}^{\prime} \notin \mathscr{P} \cup \widetilde{\mathscr{A}} \cup \widetilde{\mathscr{M}}$. Then Pl- \#CSP${ }^{2}\left(\hat{g}^{\prime}\right)$ is \#P-hard by Theorem 12.5 and we are done.

- If $g=[s, t i]^{\otimes 2 m} \pm[t, s i]^{\otimes 2 m}$, we have

$$
g^{\prime}=\partial^{m-2}(g)=\left(s^{2}-t^{2}\right)^{m-2}\left\{[s, t i]^{\otimes 4} \pm(-1)^{m-2}[t, s i]^{\otimes 4}\right\}
$$

and from $f^{\prime}$ we get $[1,0,-1]^{\otimes 2}$ on LHS by Lemma 9.20 , thus

$$
\text { Pl-Holant }\left([1,0,-1]^{\otimes 2} \cup \mathcal{E} \mathcal{Q}_{2} \mid f^{\prime}, g^{\prime}\right) \leq \operatorname{Pl} \# \operatorname{CSP}^{2}(f, g) .
$$


After a holographic transformation using $T=\left[\begin{array}{ll}1 & 0 \\ 0 & -i\end{array}\right]$, we have

$$
\begin{array}{r}
\text { Pl-Holant }\left([1,0,1]^{\otimes 2},[1,0,-1],[1,0,0,0,1], \ldots \mid \hat{f}^{\prime}, \hat{g}^{\prime}\right) \\
\equiv \text { Pl-Holant }\left([1,0,-1]^{\otimes 2} \cup \mathcal{E} \mathcal{Q}_{2} \mid f^{\prime}, g^{\prime}\right),
\end{array}
$$

where $\hat{f}^{\prime}=\left(T^{-1}\right)^{\otimes 4} f^{\prime}=[1,1]^{\otimes 4}+i^{r}[1,-1]^{\otimes 4}$ and $\hat{g}^{\prime}=\left(T^{-1}\right)^{\otimes 4} g^{\prime}$. Note that $\hat{f}^{\prime}$ satisfies a second order recurrence of type $\langle-1,0,1\rangle$. Thus $\hat{f}^{\prime} \notin \widehat{\mathscr{M}}$ by Lemma 9.11. Also note that $\mathscr{P}$ and $\widetilde{\mathscr{A}}$ are invariant under $T$, and since $g^{\prime} \in \widehat{\mathscr{M}} \backslash(\mathscr{P} \cup \widetilde{\mathscr{A}})$, we have $\hat{g^{\prime}} \in \widehat{\mathscr{M}} \backslash(\mathscr{P} \cup \widetilde{\mathscr{A}})$. In the following, we will construct $[1,0,1]^{\otimes 2}$ on RHS for

$$
\text { Pl-Holant }\left([1,0,1]^{\otimes 2},[1,0,-1],[1,0,0,0,1], \ldots \mid \hat{f}^{\prime}, \hat{g}^{\prime}\right) .
$$

Since we have $[1,0,1]^{\otimes 2}$ on LHS, we can get $\left[\partial\left(\hat{f}^{\prime}\right)\right]^{\otimes 2}=4\left[1+i^{r}, 1-\right.$ $\left.i^{r}, 1+i^{r}\right]^{\otimes 2}$ on RHS.

- If $r=0$, then we directly have $[1,0,1]^{\otimes 2}$ on RHS.

- If $r=2$, then we have $[0,1,0]^{\otimes 2}$ on RHS. Thus we can move $[1,0,1]^{\otimes 2}$ on LHS to RHS.

- If $r=1$ or 3 , then we have $[1, \pm i, 1]^{\otimes 2}$ on RHS. By connecting two copies of $[1, \pm i, 1]^{\otimes 2}$ by $[1,0,1]^{\otimes 2}$ of LHS, we have a nonzero multiple of $[0,1,0]^{\otimes 2}$ on RHS. Then we can move $[1,0,1]^{\otimes 2}$ on LHS to RHS.

From the above, we have

$$
\leq \quad \begin{gathered}
\text { Pl-Holant }\left([1,0,1]^{\otimes 2},[1,0,-1],[1,0,0,0,1], \ldots \mid \hat{f}^{\prime}, \hat{g}^{\prime},[1,0,1]^{\otimes 2}\right) \\
\text { Pl-Holant }\left([1,0,1]^{\otimes 2},[1,0,-1],[1,0,0,0,1], \ldots \mid \hat{f}^{\prime}, \hat{g}^{\prime}\right) .
\end{gathered}
$$

Note that we have all of $={ }_{4 k}$ on the LHS. Thus by Lemma 10.4,

$\operatorname{Pl}-\# \operatorname{CSP}^{2}\left(\hat{f}^{\prime}, \hat{g^{\prime}}\right) \leq \operatorname{Pl}-\operatorname{Holant}\left([1,0,1]^{\otimes 2},[1,0,-1],[1,0,0,0,1], \ldots \mid \hat{f}^{\prime},{\hat{g^{\prime}}}^{\prime},[1,0,1]^{\otimes 2}\right)$.

Recall that $\hat{g}^{\prime} \in \widehat{\mathscr{M}} \backslash(\mathscr{P} \cup \widetilde{\mathscr{A}})$ and $\hat{f}^{\prime} \notin \widehat{\mathscr{M}}$. Thus we are done by Lemma 13.3.

\subsection{Mixing with $S_{3}=\mathscr{A}^{\dagger}$}

In this subsection, we prove the No-Mixing lemma for $\mathscr{A}^{\dagger}$ with other tractable sets, namely the statements No-Mixing-(3,j), for $1 \leq j \leq 2$. Because we have already proved Lemma 14.3 and Lemma 14.6, the No-Mixing lemmas for $S_{5}=\mathscr{P}$ and $S_{4}=\mathscr{A}$ respectively, we only need to consider the mixing of $S_{3}=\mathscr{A}^{\dagger}$ with $S_{j}$ for $1 \leq j \leq 2$. Thus we may assume $f \in \mathscr{A}^{\dagger}$ and $g \in \widetilde{\mathscr{M}} \backslash \mathscr{A}^{\dagger}$. Moreover, we can assume that $f, g \notin \mathscr{P} \cup \mathscr{A}$.

Lemma 14.7 Let $\{f, g\} \subseteq\left(\bigcup_{k=1}^{3} S_{k}\right) \backslash\left(S_{4} \cup S_{5}\right)$ and $\{f, g\} \nsubseteq S_{j}$ for $1 \leq j \leq 3$. Assume that $f \in S_{3}=\mathscr{A}^{\dagger}$, then $\mathrm{Pl}-\# \operatorname{CSP}^{2}(f, g)$ is \#P-hard. 
Proof Firstly, we have $f \in \mathscr{A}^{\dagger} \backslash \mathscr{P}$, thus $f \in\left\{\left[1, \alpha,-\alpha^{2}\right],[1, \alpha]^{\otimes 2 n}+\right.$ $\left.i^{r}[1,-\alpha]^{\otimes 2 n} \mid 2 n \geq 4\right\}$ (see Fig. 24). Clearly $\left[1, \alpha,-\alpha^{2}\right]$ is not $\lambda[1,0,1]$. If $f=[1, \alpha]^{\otimes 2 n}+i^{r}[1,-\alpha]^{\otimes 2 n}$, then we have $\partial^{n-1}(f)=\left(1+\alpha^{2}\right)^{n-1}\left\{[1, \alpha]^{\otimes 2}+\right.$ $\left.i^{r}[1,-\alpha]^{\otimes 2}\right\}=\left(1+\alpha^{2}\right)^{n-1}\left[1+i^{r},\left(1-i^{r}\right) \alpha,\left(1+i^{r}\right) \alpha^{2}\right]$ which is not $\lambda[1,0,1]$. Hence we can always obtain a nonzero binary signature that is not $\lambda[1,0,1]$ from $f$.

Note that $g \in \widetilde{\mathscr{M}} \backslash(\mathscr{P} \cup \widetilde{\mathscr{A}})$. If $g \in \widehat{\mathscr{M}} \backslash(\mathscr{P} \cup \widetilde{\mathscr{A}})$, we are done by Lemma 13.3. For $g \in \widehat{\mathscr{M}} \backslash \backslash(\mathscr{P} \cup \widetilde{\mathscr{A}})$, since we have a nonzero binary signature that is not $\lambda[1,0,1]$, we are done by Lemma 13.7 .

\subsection{Mixing with $S_{2}=\widehat{\mathscr{M}}$}

In this subsection, we prove the No-Mixing lemma for $\widehat{\mathscr{M}}$ with other tractable sets. Because we have already proved Lemma 14.3, Lemma 14.6, and Lemma 14.7, the No-Mixing lemmas for $S_{5}=\mathscr{P}, S_{4}=\mathscr{A}$, and $S_{3}=\mathscr{A}^{\dagger}$ respectively, we only need to consider the No-Mixing of $S_{2}=\widehat{\mathscr{M}}$ with $S_{1}=\widehat{\mathscr{M}}^{\dagger}$.

Lemma 14.8 Let $\{f, g\} \subseteq\left(\bigcup_{k=1}^{2} S_{k}\right) \backslash\left(S_{3} \cup S_{4} \cup S_{5}\right)$ and $\{f, g\} \nsubseteq S_{j}$ for $1 \leq j \leq 2$. Then $\mathrm{Pl}$ \#CSP${ }^{2}(f, g)$ is \#P-hard.

Proof Either $f$ or $g \in \widehat{\mathscr{M}}$, otherwise $\{f, g\} \subseteq \widehat{\mathscr{M}}$. As they do not belong to $S_{3} \cup$ $S_{4} \cup S_{5}=\mathscr{P} \cup \mathscr{A}$, we have a signature in $\widehat{\mathscr{M}} \backslash(\mathscr{P} \cup \mathscr{A})$. Thus we are done by Lemma 13.3 .

By Lemma 14.3, Lemma 14.6, Lemma 14.7 and Lemma 14.8, we have the following No-Mixing theorem for two signatures with even arities.

Theorem 14.9 Let $f$ and $g$ be two symmetric signatures of even arity. If $\{f, g\} \subseteq$ $\cup_{k=1}^{5} S_{k}$ and $\{f, g\} \nsubseteq S_{j}$ for $1 \leq j \leq 5$, then $\mathrm{Pl}_{-1 C S P}(f, g)$ is \#P-hard.

\section{No-Mixing of Even Arity Signature Set}

In this section, we extend Theorem 14.9, the No-Mixing theorem for a pair of two signatures of even arity, to Theorem 15.4, the No-Mixing theorem for a set of signatures of even arity. For convenience, we explicitly list some signature sets that will be used in the proof of Theorem 15.4.

Lemma 15.1 Restricted to nonzero even arity signatures, ignoring a nonzero factor, we have

1. $\mathscr{A}^{\dagger} \cap(\mathscr{P} \cup \mathscr{A})$ is the set

$$
\left\{[1, \alpha]^{\otimes 2 n},[1,0]^{\otimes 2 n},[0,1]^{\otimes 2 n},[0,1,0],\left[1,0, \ldots, 0, i^{r}\right] \mid n \geq 1,0 \leq r \leq 3\right\} .
$$

2. $\widehat{\mathscr{M}} \cap(\mathscr{P} \cup \tilde{\mathscr{A}})$ is the set

$$
\left\{[1, \pm 1]^{\otimes 2 m},[0,1,0],[1, \pm i, 1],[1,0, \ldots, 0, \pm 1],[1, i]^{\otimes 2 n} \pm[1,-i]^{\otimes 2 n} \mid m \geq 1, n \geq 2\right\} .
$$


3. $\widehat{\mathscr{M}}^{\dagger} \cap(\mathscr{P} \cup \widetilde{\mathscr{A}})$ is the set

$$
\left\{[1, \pm i]^{\otimes 2 m},[0,1,0],[1, \pm 1,-1],[1,0, \ldots, 0, \pm 1],[1,1]^{\otimes 2 n} \pm[1,-1]^{\otimes 2 n} \mid m \geq 1, n \geq 2\right\} .
$$

4. $\bigcap_{3 \leq k \leq 5} S_{k}$ is the set $\left\{[1,0]^{\otimes 2 n},[0,1]^{\otimes 2 n},[0,1,0],\left[1,0, \ldots, 0, i^{r}\right] \mid n \geq 1,0 \leq\right.$ $r \leq 3\}$.

5. $\bigcap_{1 \leq k \leq 5} S_{k}=\bigcap_{2 \leq k \leq 5} S_{k}$ is the set $\{[0,1,0],[1,0, \ldots, 0, \pm 1]\}$.

Proof For all five cases, it is easy to show that the listed signatures in the displayed set are indeed members of the respective stated intersection, bear in mind that the signatures all have even arity. E.g., the signature $f=\left[1,0, \ldots, 0, i^{r}\right]$ is clearly in $\mathscr{P}$ (as well as $\mathscr{A}$ ), and it has even arity $2 n$, and thus under the transformation $T=\left[\begin{array}{ll}1 & 0 \\ 0 & \alpha\end{array}\right],\left(T^{-1}\right)^{2 n} f=\left[1,0, \ldots, 0, i^{s}\right] \in \mathscr{A}$, for some $0 \leq s \leq 3$. Thus $f \in \mathscr{A}^{\dagger}$.

In the following, we prove that if $f$ is a nonzero signature of even arity and is in the stated intersection then it is among the listed types.

1. (a.) Firstly, suppose that $f \in \mathscr{A}^{\dagger} \cap(\mathscr{P} \cup \mathscr{A})$ is degenerate, i.e., $f=[a, b]^{2 n}$. If $f=[1,0]^{2 n}$ or $[0,1]^{2 n}$ up to a nonzero scalar, then $f$ is among the listed. Suppose $a b \neq 0$. Then up to a nonzero scalar, $f=[1, \xi]^{2 n}$, for some $\xi \neq 0$. By $f \in \mathscr{A}^{\dagger}$, we have $\left[\begin{array}{ll}1 & 0 \\ 0 & \alpha\end{array}\right]^{2 n} f=[1, \alpha \xi]^{2 n} \in \mathscr{A}$. Thus $(\alpha \xi)^{4}=1$, i.e., $\xi^{4}=-1$. So $f$ is among the listed types.

(b.) If $f \in \mathscr{A}^{\dagger} \cap(\mathscr{P} \cup \mathscr{A})$ is a non-degenerate binary signature, by $f \in \mathscr{A}^{\dagger}$ and Lemma 9.8, we have $f=\left[1, \alpha,-\alpha^{2}\right]$, or $[0,1,0]$, or $[1,0, \rho]$ up to a scalar, where $\alpha^{4}=-1, \rho^{4}=1$. Note that $\left[1, \alpha,-\alpha^{2}\right] \notin \mathscr{P} \cup \mathscr{A}$ by Corollary 9.9. Thus $f=[0,1,0]$ or $[1,0, \rho]$; these are among the listed types.

(c.) If $f \in \mathscr{A}^{\dagger} \cap(\mathscr{P} \cup \mathscr{A})$ is non-degenerate and has arity $2 n \geq 4$, by $f \in \mathscr{A}^{\dagger}$ and Lemma 9.11, $f$ has type $\langle 0,1,0\rangle$ or $\langle 1,0, \pm i\rangle$ and the second-order recurrence relation is unique up to a scalar. If $f$ has type $\langle 1,0, \pm i\rangle$, then $f \notin \mathscr{P} \cup \mathscr{A}$ by Lemma 9.11. This contradicts that $f \in \mathscr{A}^{\dagger} \cap(\mathscr{P} \cup \mathscr{A})$. If $f$ has type $\langle 0,1,0\rangle$, then $f=[1,0, \ldots, 0, x]$ with $x \neq 0$ up to a nonzero scalar, because $f$ is non-degenerate. Moreover, if $x^{4} \neq 1$, bear in mind that $f$ has even arity, then $f \notin \mathscr{A}^{\dagger}$ and this contradicts that $f \in \mathscr{A}^{\dagger} \cap(\mathscr{P} \cup \mathscr{A})$. Hence $x^{4}=1$ and $f=\left[1,0, \ldots, 0, i^{r}\right]$, for some $0 \leq r \leq 3$; this is among the listed types.

Summarizing, we proved that if $f \in \mathscr{A}^{\dagger} \cap(\mathscr{P} \cup \mathscr{A})$ then $f$ is among the listed types.

2. (a.) Suppose $f \in \widehat{\mathscr{M}} \cap(\mathscr{P} \cup \widetilde{\mathscr{A}})$ is a nonzero degenerate signature, i.e., $f=$ $[a, b]^{\otimes 2 n}$. By $f \in \widehat{\mathscr{M}}$ we have $\left[\begin{array}{ll}1 & 1 \\ 1 & -1\end{array}\right]^{\otimes 2 n} f=[a+b, a-b]^{\otimes 2 n} \in$ $\mathscr{M}$, which must satisfy the parity constraints. Thus $a= \pm b$ and $f=$ $[1, \pm 1]^{\otimes 2 n}$ up to a nonzero scalar. 
(b.) If $f \in \widehat{\mathscr{M}} \cap(\mathscr{P} \cup \widetilde{\mathscr{A}})$ is a non-degenerate binary signature, by $f \in \widehat{\mathscr{M}}$ and Lemma 9.8, we have $f=[0,1,0]$, or $[1, b, 1]$, or $[1,0,-1]$ up to a nonzero scalar. If $f=[1, b, 1]$ and $b^{4} \neq 0,1$, then $f \notin \mathscr{P} \cup \widetilde{\mathscr{A}}$, by Corollary 9.9. This contradicts that $f \in \widehat{\mathscr{M} \cap}(\mathscr{P} \cup \widetilde{\mathscr{A}})$. Thus, $f=[0,1,0]$, $[1,0,1],\left[1, i^{r}, 1\right]$ or $[1,0,-1]$, where $0 \leq r \leq 3$. Note that if $r=0$ or 2 , then $\left[1, i^{r}, 1\right]=[1, \pm 1,1]=[1, \pm 1]^{\otimes 2}$. Thus all these binary signatures are in the listed types.

(c.) If $f \in \widehat{\mathscr{M}} \cap(\mathscr{P} \cup \widetilde{\mathscr{A}})$ is non-degenerate and has arity $2 n \geq 4$, by $f \in \widehat{\mathscr{M}}$ and Lemma 9.11, $f$ has type $\langle 0,1,0\rangle$ or $\langle 1, c, 1\rangle$, and the second-order recurrence relation is unique up to a scalar. If $f$ has type $\langle 1, c, 1\rangle$ with $c \neq 0$, then $f \notin \mathscr{P} \cup \widetilde{\mathscr{A}}$ by Lemma 9.11 and this contradicts that $f \in \widehat{\mathscr{M}} \cap(\mathscr{P} \cup \widetilde{\mathscr{A}})$. If $f$ has type $\langle 1,0,1\rangle$, then there exist constants $x$ and $y$ such that $f=x[1, i]^{\otimes 2 n}+y[1,-i]^{\otimes 2 n}$. By non-degeneracy, we get $x y \neq \sim$, and by its type $\langle 1,0,1\rangle, f \notin \mathscr{P}$ by Lemma 9.11. Thus $f \in \tilde{A}$. In fact by Lemma 9.11 and its type $\langle 1,0,1\rangle, f \notin \mathscr{A}^{\dagger} \backslash \mathscr{P}$, thus it follows that $f \in \mathscr{A} \backslash \mathscr{P}$. Then there are two possibilities: Either $f=\left[\begin{array}{cc}1 & 1 \\ 1 & -1\end{array}\right]^{\otimes 2 n}\left\{[1,0]^{\otimes 2 n}+i^{r}[0,1]^{\otimes 2 n}\right\}$, or $f=\left[\begin{array}{ll}1 & 1 \\ i & -i\end{array}\right]^{\otimes 2 n}\left\{[1,0]^{\otimes 2 n}+i^{r}[0,1]^{\otimes 2 n}\right\}$, up to a nonzero scalar, where $0 \leq r \leq 3$. However, the first possibility is a contradiction to $f \in \widehat{\mathscr{M}}$, because $[1,0]^{\otimes 2 n}+i^{r}[0,1]^{\otimes 2 n} \notin \mathscr{M}$, for arity $2 n \geq 4$, by Proposition 2.8. Thus $f=[1, i]^{\otimes 2 n}+i^{r}[1,-i]^{\otimes 2 n}$ up to a nonzero scalar, for some $0 \leq r \leq 3$. If $f=[1, i]^{\otimes 2 n} \pm i[1,-i]^{\otimes 2 n}$, then $\left[\begin{array}{ll}1 & 1 \\ 1 & -1\end{array}\right]^{\otimes 2 n} f$ is $( \pm i)(1-i)^{2 n}\left\{[1, i]^{\otimes 2 n} \mp i(-1)^{n}[1,-i]^{\otimes 2 n}\right\}$. This is a nonzero multiple of $[1, i]^{\otimes 2 n} \pm i[1,-i]^{\otimes 2 n}$, which does not satisfy the parity constraints, and hence not in $\mathscr{M}$. So $f$ is not in $\widehat{\mathscr{M}}$. Hence $f=[1, i]^{\otimes 2 n} \pm[1,-i]^{\otimes 2 n}$, which is among the listed types.

If $f$ has type $\langle 0,1,0\rangle$, then $f=[1,0, \ldots, 0, x]$ with $x \neq 0$, up to a nonzero scalar. By $f \in \widehat{\mathscr{M}}$ and Lemma 9.16, we have $x^{2}=1$. Thus $f=$ $[1,0, \ldots, 0, \pm 1]$, which is among the listed types.

Summarizing, we proved that if $f \in \widehat{\mathscr{M}} \cap(\mathscr{P} \cup \widetilde{\mathscr{A}})$, then $f$ is among the listed types.

3. Note that $\mathscr{P} \cup \tilde{\mathscr{A}}$ is unchanged under the transformation by $\left[\begin{array}{ll}1 & 0 \\ 0 & i\end{array}\right]$. Thus

$$
\widehat{\mathscr{M}^{\dagger}} \cap(\mathscr{P} \cup \widetilde{\mathscr{A}})=\left[\begin{array}{ll}
1 & 0 \\
0 & i
\end{array}\right]\{\widehat{\mathscr{M}} \cap(\mathscr{P} \cup \widetilde{\mathscr{A}})\} .
$$

Then the proof of this case follows from the previous case by a transformation using $\left[\begin{array}{ll}1 & 0 \\ 0 & i\end{array}\right]$.

4. If $f \in \bigcap_{k=3}^{5} S_{k}$, then a fortiori, $f \in \mathscr{A}^{\dagger} \cap(\mathscr{P} \cup \mathscr{A})$. This implies that $f \in\left\{[1, \alpha]^{\otimes 2 n},[1,0]^{\otimes 2 n},[0,1]^{\otimes 2 n},[0,1,0],\left[1,0, \ldots, 0, i^{r}\right] \mid n \geq 1,0 \leq r \leq 3\right\}$. 
Note that $[1, \alpha]^{\otimes 2 m} \notin \mathscr{A}$. Thus $f=[1,0]^{\otimes 2 n}$, or $[0,1]^{\otimes 2 n}$, or $[0,1,0]$, or $\left[1,0, \ldots, 0, i^{r}\right]$. All of these four types are among the listed.

5. We already have

$$
\{[0,1,0],[1,0, \ldots, 0, \pm 1]\} \subseteq \bigcap_{1 \leq k \leq 5} S_{k} \subseteq \bigcap_{2 \leq k \leq 5} S_{k}
$$

If $f \in \bigcap_{k=2}^{5} S_{k}$, then $f \in \bigcap_{k=3}^{5} S_{k}$. This implies that

$$
f \in\left\{[1,0]^{\otimes 2 n},[0,1]^{\otimes 2 n},[0,1,0],\left[1,0, \ldots, 0, i^{r}\right] \mid n \geq 1,0 \leq r \leq 3\right\} .
$$

Moreover, if $f=[1,0]^{\otimes 2 n}$, or $[0,1]^{\otimes 2 n}$ or $[1,0, \ldots, 0, \pm i]$, then $f \notin \widehat{\mathscr{M}}$, because $\left[\begin{array}{ll}1 & 1 \\ 1 & -1\end{array}\right]^{\otimes 2 n} f$ does not satisfy the parity constraints. Hence $f=$ $[0,1,0]$, or $[1,0, \ldots, 0, \pm 1]$, and both types are among the listed.

We state the following simple lemma which allows us to replace a signature set $\mathcal{F}$ in the proof of the No-Mixing Theorem by a smaller set $\mathcal{F}^{\prime}$ that subtracts from $\mathcal{F}$ those signatures that belong to all common tractable signature sets.

Lemma 15.2 Let $\mathcal{F}$ be a set of symmetric signatures such that for all $1 \leq k \leq 5$, $\mathcal{F} \nsubseteq S_{k}$. Let $\mathcal{F}^{\prime}=\mathcal{F} \backslash\left(\bigcap_{k=1}^{5} S_{k}\right)$, the set of signatures in $\mathcal{F}$ excluding those that belong to all five tractable signature sets. Then for all $1 \leq k \leq 5, \mathcal{F}^{\prime} \nsubseteq S_{k}$ and $\operatorname{Pl}-\# \operatorname{CSP}^{2}\left(\mathcal{F}^{\prime}\right) \leq \operatorname{Pl}$ \#CSP${ }^{2}(\mathcal{F})$.

Proof Suppose for some $1 \leq k \leq 5, \mathcal{F}^{\prime} \subseteq S_{k}$, then clearly $\mathcal{F} \subseteq S_{k}$. The reduction is trivial since $\mathcal{F}^{\prime} \subseteq \mathcal{F}$.

Suppose $\mathcal{F}$ is as given in Lemma 15.2, and $\mathcal{F} \cap\left(\bigcup_{k=1}^{5} S_{k}\right) \neq \emptyset$, i.e., there exists some signature in $\mathcal{F}$ outside of all five tractable signature sets. Let $j=\min \{k \mid$ $\left.\mathcal{F} \cap S_{k} \neq \emptyset, 1 \leq k \leq 5\right\}$. Then $j$ is well defined. Then by definition and De Morgan's laws $\mathcal{F}^{\prime}=\mathcal{F} \backslash\left(\bigcap_{k=j}^{5} S_{k}\right)$, is also the set of signatures in $\mathcal{F}$ excluding those that belong to all signature sets $S_{k}$ for $j \leq k \leq 5$. By Lemma 15.2 we have $\mathcal{F}^{\prime} \nsubseteq S_{k}$, for $j \leq k \leq 5, \mathcal{F}^{\prime} \cap S_{k}=\emptyset$ for $1 \leq k<j$ (by definition), and $\mathrm{Pl}-\# \mathrm{CSP}^{2}\left(\mathcal{F}^{\prime}\right) \leq$ $\mathrm{Pl}-\# \operatorname{CSP}^{2}(\overline{\mathcal{F}})$. For ease of application we state this as a corollary.

Corollary 15.3 Let $\mathcal{F}$ be a set of symmetric signatures such that for all $1 \leq k \leq 5$, $\mathcal{F} \nsubseteq S_{k}$. Furthermore suppose $\mathcal{F} \cap\left(\bigcup_{k=1}^{5} S_{k}\right) \neq \emptyset$ and let $j=\min \left\{k \mid \overline{\mathcal{F}} \cap S_{k} \neq\right.$ $\emptyset, 1 \leq k \leq 5\}$. Let $\mathcal{F}^{\prime}=\mathcal{F} \backslash\left(\bigcap_{k=j}^{5} S_{k}\right)$. Then for all $j \leq k \leq 5, \mathcal{F}^{\prime} \nsubseteq S_{k}$ and $\operatorname{Pl}-\# \operatorname{CSP}^{2}\left(\mathcal{F}^{\prime}\right) \leq \operatorname{Pl}-\# \operatorname{CSP}^{2}(\mathcal{F})$.

$$
\text { Recall that } S_{1}=\widehat{\mathscr{M}}, S_{2}=\widehat{\mathscr{M}}^{\dagger}, S_{3}=\mathscr{A}^{\dagger}, S_{4}=\mathscr{A} \text { and } S_{5}=\mathscr{P} \text {. }
$$

Theorem 15.4 Let $\mathcal{F} \subseteq \bigcup_{k=1}^{5} S_{k}$ be a set of symmetric signatures of even arities. If $\mathcal{F} \subseteq S_{k}$ for some $1 \leq k \leq 5$, then $\mathrm{Pl}-\# \operatorname{CSP}^{2}(\mathcal{F})$ is tractable. Otherwise, $\mathrm{Pl}-\# \operatorname{CSP}^{2}(\overline{\mathcal{F}})$ is \#P-hard. 
Proof If $\mathcal{F} \subseteq S_{k}$ for some $1 \leq k \leq 5$, then tractability follows from the definition of $\mathscr{P}$-transformability, $\mathscr{A}$-transformability and $\mathscr{M}$-transformability.

Now suppose $\mathcal{F} \nsubseteq S_{k}$ for all $1 \leq k \leq 5$. We first replace $\mathcal{F}$ by $\mathcal{F}^{\prime}=\mathcal{F} \backslash$ $\left(\bigcap_{k=1}^{5} S_{k}\right)$. This also excludes the identically 0 signature. By Lemma 15.2 , we still have $\mathcal{F}^{\prime} \nsubseteq S_{k}$ for $1 \leq k \leq 5$, and we only need to prove $\mathrm{Pl}$-\#CSP${ }^{2}\left(\mathcal{F}^{\prime}\right)$ is \#P-hard.

We will treat the tractable sets in the order $S_{1}, S_{2}, \ldots, S_{5}$, starting with $S_{1}=\widehat{\mathscr{M}}$.

1. Suppose that $\mathcal{F}^{\prime} \cap S_{1} \neq \emptyset$.

Let $\mathcal{G}_{1}=\mathcal{F}^{\prime} \cap S_{1}$, and $\mathcal{H}_{1}=\mathcal{F}^{\prime} \backslash S_{1}$. Then $\mathcal{G}_{1} \neq \emptyset$, and since $\mathcal{F}^{\prime} \nsubseteq S_{1}$ we also have $\mathcal{H}_{1} \neq \emptyset$. If there exists $g \in \mathcal{G}_{1}$ such that $g \in \widehat{\mathscr{M} \backslash}(\mathscr{P} \cup \widetilde{\mathscr{A}})$, then we are done by Lemma 13.3. Otherwise, $\mathcal{G}_{1} \subseteq \widehat{\mathscr{M}} \cap(\mathscr{P} \cup \mathscr{\mathscr { A }})$. Then by the forms given in Lemma 15.1, ignoring nonzero scalars, $\mathcal{G}_{1} \subseteq\left\{[1, \pm 1]^{\otimes 2 m},[1, \pm i, 1],[1, i]^{\otimes 2 n} \pm\right.$ $\left.[1,-i]^{\otimes 2 n} \mid m \geq 1, n \geq 2\right\}$. Note that we have excluded $\bigcap_{k=1}^{5} S_{k}$ in $\mathcal{F}^{\prime}$, hence also in $\mathcal{G}_{1}$. By Lemma $15.1,[1,0, \ldots, 0, \pm 1],[0,1,0] \notin \mathcal{F}^{\prime}$.

If $[1, \pm 1]^{\otimes 2 m} \in \mathcal{G}_{1}$ for some $m \geq 1$, then we can construct $\partial^{m-1}\left([1, \pm 1]^{\otimes 2 m}\right)=2^{m-1}[1, \pm 1]^{\otimes 2}$, and we are done by Lemma 10.3.

Otherwise, by the forms in

$$
\mathcal{G}_{1} \subseteq\left\{[1, \pm i, 1],[1, i]^{\otimes 2 n} \pm[1,-i]^{\otimes 2 n} \mid n \geq 2\right\}
$$

we have $\mathcal{G}_{1} \subseteq \mathscr{A}$. If $\mathcal{H}_{1} \subseteq \mathscr{A}$, then we would have $\mathcal{F}^{\prime} \subseteq \mathscr{A}$, a contradiction. Thus $\mathcal{H}_{1} \nsubseteq \mathscr{\mathscr { A }}$. Thus there exists $h \in \mathcal{H}_{1} \backslash \mathscr{A}$. By definition of $\mathcal{H}_{1}, h \notin \widehat{\mathscr{M}}$. Also $\mathcal{H}_{1} \subseteq \cup_{k=1}^{5} S_{k}$, thus $h \in\left(\mathscr{P} \cup \mathscr{A}^{\dagger} \cup \widehat{\mathscr{M}}^{\dagger}\right) \backslash(\mathscr{A} \cup \widehat{\mathscr{M}})$. By the forms of signatures in the nonempty set $\mathcal{G}_{1}$ in $(15.27)$ we have $\mathcal{G}_{1} \cap\left(\mathscr{P} \cup \mathscr{A}^{\dagger} \cup \widehat{\mathscr{M}^{\dagger}}\right)=\emptyset$. To check this: for the binary $[1, \pm i, 1]$, we apply Lemma 9.8; for $[1, i]^{\otimes 2 n} \pm[1,-i]^{\otimes 2 n}$ we use its second-order recurrence of type $\langle 1,0,1\rangle$ and then we apply Lemma 9.11. Thus Pl-\#CSP ${ }^{2}\left(\mathcal{F}^{\prime}\right)$ is \#P-hard by Theorem 14.9.

2. We have $\mathcal{F}^{\prime} \cap S_{1}=\emptyset$. We replace $\mathcal{F}^{\prime}$ by $\mathcal{F}^{\prime \prime}=\mathcal{F}^{\prime} \backslash\left(\bigcap_{k=2}^{5} S_{k}\right)$. By Corollary 15.3, we still have $\mathcal{F}^{\prime \prime} \nsubseteq S_{k}$ for $2 \leq k \leq 5, \mathcal{F}^{\prime \prime} \cap S_{1}=\emptyset$, and we only need to prove $\mathrm{Pl}-\# \mathrm{CSP}^{2}\left(\mathcal{F}^{\prime \prime}\right)$ is \#P-hard.

Now suppose that $\mathcal{F}^{\prime \prime} \cap S_{2} \neq \varnothing$.

By Lemma 15.1, $[1,0, \ldots, 0, \pm 1],[0,1,0] \notin \mathcal{F}^{\prime \prime}$.

Let $\mathcal{G}_{2}=\mathcal{F}^{\prime \prime} \cap S_{2}$ and $\mathcal{H}_{2}=\mathcal{F}^{\prime \prime} \backslash S_{2}$. Both $\mathcal{G}_{2}, \mathcal{H}_{2} \neq \emptyset\left(\mathcal{G}_{2} \neq \emptyset\right.$ by assumption, $\mathcal{H}_{2} \neq \emptyset$ by $\left.\mathcal{F}^{\prime \prime} \nsubseteq S_{2}\right)$, and we also have $\mathcal{H}_{2} \cap \widetilde{\mathscr{M}}=\emptyset$ by definition. Thus there exists $h \in \mathcal{H}_{2} \backslash \widetilde{\mathscr{M}}$. If there exists $g \in \mathcal{G}_{2}$ such that $g \in \widehat{\mathscr{M}}^{\dagger} \backslash(\mathscr{P} \cup \widetilde{\mathscr{A}})$, then $\mathrm{Pl}$-\#CSP${ }^{2}(f, g)$ is \#P-hard by Theorem 14.9.

Otherwise, $\mathcal{G}_{2} \subseteq \widehat{\mathscr{M}}^{\dagger} \cap(\mathscr{P} \cup \widetilde{\mathscr{A}})$. Then $\mathcal{G}_{2} \subseteq$ $\left\{[1, \pm i]^{\otimes 2 m},[1, \pm 1,-1],[1,1]^{\otimes 2 n} \pm[1,-1]^{\otimes 2 n} \mid m \geq 1, n \geq 2\right\}$ by Lemma 15.1. By its form $\mathcal{G}_{2} \subseteq \mathscr{A}$. If $\mathcal{H}_{2} \subseteq \mathscr{A}$, then we would have $\mathcal{F}^{\prime \prime} \subseteq \mathscr{A}$, a contradiction. Thus $\mathcal{H}_{2} \nsubseteq \mathscr{A}$. Hence there exists $h^{\prime} \in \mathcal{H}_{2} \backslash \mathscr{A}$. By definition of $\mathcal{H}_{2}, h^{\prime} \notin \widetilde{\mathscr{M}}$. As $\mathcal{F}^{\prime \prime} \subseteq \bigcup_{k=2}^{5} S_{k}, h^{\prime} \in\left(\mathscr{P} \cup \mathscr{A}^{\dagger}\right) \backslash(\mathscr{A} \cup \widetilde{\mathscr{M}})$. We note that both $[1, \pm 1,-1]$ and $[1,1]^{\otimes 2 n} \pm[1,-1]^{\otimes 2 n}$, for any $n \geq 2$, are not in $\mathscr{P} \cup \mathscr{A}^{\dagger}$. To see this for $[1, \pm 1,-1]$ we apply Corollary 9.9. To see this for $[1,1]^{\otimes 2 n} \pm[1,-1]^{\otimes 2 n}$ with $n \geq 2$, we note its recurrence type $\langle-1,0,1\rangle$ and then apply Lemma 9.11 . Now if $\mathcal{G}_{2}$ includes either $[1, \pm 1,-1]$ 
or $[1,1]^{\otimes 2 n} \pm[1,-1]^{\otimes 2 n}$ for some $n \geq 2$, then $\mathrm{Pl}-\# \mathrm{CSP}^{2}\left(\mathcal{F}^{\prime \prime}\right)$ is \#P-hard by Theorem 14.9.

We are left with the case where the nonempty set $\mathcal{G}_{2} \subseteq\left\{[1, \pm i]^{\otimes 2 m} \mid m \geq 1\right\}$. By its form $\mathcal{G}_{2} \subseteq \mathscr{P} \cap \mathscr{A} \cap \widehat{\mathscr{M}}^{\dagger}$ and $\mathcal{G}_{2} \cap \mathscr{A}^{\dagger} \equiv \emptyset$. If there exists $\bar{h}^{\prime \prime} \in$ $\mathcal{H}_{2} \backslash(\mathscr{A} \cup \mathscr{P})$, then by definition of $\mathcal{H}_{2}$ this $h^{\prime \prime} \notin \widetilde{\mathscr{M}}$ as well, and we conclude that $\mathrm{Pl}-\# \mathrm{CSP}^{2}\left(\mathcal{F}^{\prime \prime}\right)$ is \#P-hard by Theorem 14.9.

So we may assume $\mathcal{H}_{2} \subseteq \mathscr{A} \cup \mathscr{P}$. If $\mathcal{H}_{2} \subseteq \mathscr{A}$, then we would have $\mathcal{F}^{\prime \prime} \subseteq \mathscr{A}$, a contradiction. Thus there exists $h^{\prime \prime \prime} \in\left(\mathcal{H}_{2} \cap \mathscr{P}\right) \backslash \mathscr{A}$. Considering the forms of signatures in $\mathscr{P} \backslash \mathscr{A}$, it takes the form $h^{\prime \prime \prime}=[a, b]^{\otimes 2 n}$ with $a^{4} \neq b^{4}, a b \neq 0$, or $h^{\prime \prime \prime}=[1,0, \ldots, 0, x]$ of arity $2 n$, with $x^{4} \neq 0,1$, for some $n \geq 1$. Taking $h^{\prime \prime \prime \prime}=\partial^{n-1}\left(h^{\prime \prime \prime}\right)$, we get a nonzero multiple of either $[a, b]^{\otimes 2}$ or $[1,0, x]$. Then taking $\partial_{h^{\prime \prime \prime \prime}}^{m-1}\left([1, \pm i]^{\otimes 2 m}\right)$, for some $m \geq 1$, where $[1, \pm i]^{\otimes 2 m} \in \mathcal{G}_{2}$ which is nonempty, we get a nonzero multiple of $[1, \pm i]^{\otimes 2}$, and we are done by Lemma 10.3.

3. Now we have $\mathcal{F}^{\prime \prime} \cap S_{2}=\emptyset$.

We replace $\mathcal{F}^{\prime \prime}$ by $\mathcal{F}^{\prime \prime \prime}=\mathcal{F}^{\prime \prime} \backslash\left(\bigcap_{k=3}^{5} S_{k}\right)$. By Corollary 15.3, we still have $\mathcal{F}^{\prime \prime \prime} \nsubseteq S_{k}$ for $3 \leq k \leq 5, \mathcal{F}^{\prime \prime \prime} \cap\left(S_{1} \cup S_{2}\right)=\emptyset$, and we only need to prove $\mathrm{Pl}-\# \operatorname{CSP}^{2}\left(\mathcal{F}^{\prime \prime \prime}\right)$ is \#P-hard.

Suppose that $\mathcal{F}^{\prime \prime \prime} \cap S_{3} \neq \emptyset$.

By Lemma 15.1, as $\mathcal{F}^{\prime \prime \prime} \cap \bigcap_{k=3}^{5} S_{k}=\varnothing$, the following signatures $\left[1,0, \ldots, 0, i^{r}\right]$ of arity $2 n,[0,1,0],[1,0]^{\otimes 2 n},[0,1]^{\otimes 2 n}$ all do not belong to $\mathcal{F}^{\prime \prime \prime}$, for any $0 \leq r \leq 3$ and any $n \geq 1$.

Let $\mathcal{G}_{3}=\mathcal{F}^{\prime \prime \prime} \cap S_{3}, \mathcal{H}_{3}=\mathcal{F}^{\prime \prime \prime} \backslash S_{3}$. Both $\mathcal{G}_{3}, \mathcal{H}_{3} \neq \emptyset\left(\mathcal{G}_{3} \neq \emptyset\right.$ by assumption, $\mathcal{H}_{3} \neq \emptyset$ by $\left.\mathcal{F}^{\prime \prime \prime} \nsubseteq S_{3}\right)$. Thus there exists $h \in \mathcal{H}_{3}$ such that $h \in(\mathscr{P} \cup \mathscr{A}) \backslash$ $\left(\mathscr{A}^{\dagger} \cup \widetilde{\mathscr{M}}\right)$. By definition $\mathcal{G}_{3} \subseteq S_{3}=\mathscr{A}^{\dagger}$. If there exists $g \in \mathcal{G}_{3}$ such that $g \in \mathscr{A}^{\dagger} \backslash \mathscr{P}$, then by Corollary 9.12, $g \notin \mathscr{A}$. Thus $\mathrm{Pl}$-\#CSP${ }^{2}(g, h)$ is \#P-hard by Theorem 14.9 .

Otherwise, we have $\mathcal{G}_{3} \subseteq \mathscr{A}^{\dagger} \cap \mathscr{P}$. Thus we have $\mathcal{G}_{3} \subseteq\left\{[1, \alpha]^{\otimes 2 m} \mid m \geq\right.$ $1\}$. Note that by Lemma 15.1 , we have excluded $\left[1,0, \ldots, 0, i^{r}\right]$ of arity $2 n$, $[0,1,0],[1,0]^{\otimes 2 n},[0,1]^{\otimes 2 n}$ which are all in $\bigcap_{k=3}^{5} S_{k}$. (See Fig. 24. Note that in the diagram of Fig. 24 we excluded the tensor products of unary signatures, as explained in the caption of Fig. 24.)

Then we have $\partial^{m-1}\left([1, \alpha]^{\otimes 2 m}\right)=\left(1+\alpha^{2}\right)[1, \alpha]^{\otimes 2}$ and we are done by Lemma 10.3.

4. Finally we have $\mathcal{F}^{\prime \prime \prime} \cap S_{3}=\emptyset$.

We have $\mathcal{F}^{\prime \prime \prime} \nsubseteq S_{k}$ for $4 \leq k \leq 5, \mathcal{F}^{\prime \prime \prime} \cap\left(S_{1} \cup S_{2} \cup S_{3}\right)=\emptyset$, and thus $\mathcal{F}^{\prime \prime \prime} \subseteq S_{4} \cup S_{5}$. Then we are done directly by Theorem 14.9.

\section{Dichotomy Theorem for an Even-Arity Signature}

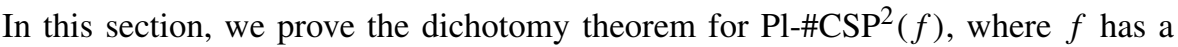
general even arity $2 n$. If $2 n=2$ or 4 , then it has been proved in Theorem $9.21^{\prime}$ and Theorem 12.5 respectively. Thus we will assume $2 n \geq 6$. 
The next simple lemma is to determine if a symmetric signature satisfies a secondorder recurrence relation. In the following proof, we often argue that a signature $f$ does not belong to $\mathscr{P} \cup \widetilde{\mathscr{A}} \cup \widetilde{\mathscr{M}}$ by Lemma 9.11. And we show that Lemma 9.11 applies by showing that $f$ does not satisfy any second-order recurrence relation.

Lemma 16.1 For a symmetric signature $f=\left[f_{0}, f_{1}, \ldots, f_{n}\right]$, let $M_{f}=$ $\left[\begin{array}{lll}f_{0} & f_{1} & f_{2} \\ f_{1} & f_{2} & f_{3} \\ \vdots & \vdots & \vdots \\ f_{n-2} & f_{n-1} & f_{n}\end{array}\right]$, then $f$ satisfies a second-order recurrence relation iff $\operatorname{rank}\left(M_{f}\right) \leq 2$.

Proof The signature $f$ satisfies a second-order recurrence relation $a f_{k}+b f_{k+1}+$ $c f_{k+2}=0$ for $0 \leq k \leq n-2$ iff the linear system $M_{f} X=0$ has a nonzero solution $(a, b, c)^{T}$ iff $\operatorname{rank}\left(M_{f}\right) \leq 2$.

We often use the following argument to prove hardness: Firstly, we prove $f \notin$ $\mathscr{P} \cup \widetilde{\mathscr{A}} \cup \widetilde{\mathscr{M}}$ using Lemma 16.1. Moreover, if we can get $[1, \xi]^{\otimes 2}$ in $\operatorname{Pl}^{-\# C^{2}}{ }^{2}(f)$ for some $\xi \neq 0$, then Pl-\#CSP${ }^{2}\left(f_{\mathcal{2}}[1, \xi]^{\otimes 2}\right)$ is \#P-hard by Lemma 10.3. Or if we can

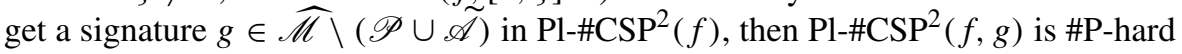
by Lemma 13.3 .

The next three lemmas are some special cases of Theorem 16.5 which is the main result of this section. We prove these lemmas separately to facilitate the presentation of the proof of Theorem 16.5.

Lemma 16.2 Suppose $a b \neq 0$ and $f=[1, a, 0,-a, 0, a, b]$, then $\operatorname{Pl}_{-} \# C_{S P}^{2}(f)$ is \#P-hard.

Proof Note that $M_{f}=\left[\begin{array}{lll}f_{0} & f_{1} & f_{2} \\ f_{1} & f_{2} & f_{3} \\ f_{2} & f_{3} & f_{4} \\ f_{3} & f_{4} & f_{5} \\ f_{4} & f_{5} & f_{6}\end{array}\right]=\left[\begin{array}{lll}1 & a & 0 \\ a & 0 & -a \\ 0 & -a & 0 \\ -a & 0 & a \\ 0 & a & b\end{array}\right]$ has rank 3. Thus $f$ does not satisfy any second-order recurrence relation by Lemma 16.1. So $f \notin \mathscr{P} \cup \widetilde{\mathscr{A}} \cup \widetilde{\mathscr{M}}$ by Lemma 9.11 .

Moreover, we have $\partial_{=4}(f)=[1,2 a, b]$. If $[1,2 a, b]$ is degenerate, then $[1,2 a, b]=[1,2 a]^{\otimes 2}$. We are done since $\operatorname{Pl} \# \operatorname{CSP}^{2}\left(f,[1,2 a]^{\otimes 2}\right)$ is \#P-hard by Lemma 10.3. Otherwise,

- $\quad$ For $b^{4} \neq 1$, we have $[1,2 a, b] \notin \mathscr{P} \cup \widetilde{\mathscr{A}} \cup \widetilde{\mathscr{M}}$ by Corollary 9.9 and Lemma 9.8 . Thus Pl-\#CSP${ }^{2}([1,2 a, b])$ is \#P-hard by Theorem 9.21' and we are done.

- For $b^{2}=-1$, we have $\partial_{[1,2 a, b]}^{2}(=6)=[1,0,-1]$ on the left and we have $f^{\prime}=\partial_{[1,0,-1]}(f)=[1,2 a, 0,-2 a,-b]$. Note that $f^{\prime}$ is redundant and the determinant of its compressed signature matrix is $4(b-1) a^{2} \neq 0$. Thus Pl-\#CSP ${ }^{2}\left(f^{\prime}\right)$, being equivalent to Pl-Holant $\left(f^{\prime}, \mathcal{E} \mathcal{Q}_{2}\right)$ (see (2.2)), is \#P-hard by Lemma 9.25 and we are done. 
- For $b^{2}=1$, if $(2 a)^{4} \neq 1$, then we have $[1,2 a, b] \in \widetilde{\mathscr{M}} \backslash(\mathscr{P} \cup \widetilde{\mathscr{A}})$ by Lemma 9.14. Thus Pl-\#CSP${ }^{2}(f,[1,2 a, b])$ is \#P-hard by Lemma 13.3 and Lemma 13.7 and we are done.

Otherwise, we have $(2 a)^{4}=1$. This implies that $(2 a)^{2}= \pm b$. Since $[1,2 a, b]$ is non-degenerate, we have $(2 a)^{2} \neq b$, thus $(2 a)^{2}=-b$. Moreover, we have $f^{\prime \prime}=\partial_{[1,2 a, b]}(f)=\left[1+(2 a)^{2},(1-b) a,-(2 a)^{2},-(1-b) a, b^{2}+(2 a)^{2}\right]$. Note that $f^{\prime \prime}=[0,0,1,0,0]$ for $b=1$ and $f^{\prime \prime}=[2, \pm 1,-1, \mp 1,2]$ for $b=-1$. Both of $[0,0,1,0,0]$ and $[2, \pm 1,-1, \mp 1,2]$ are redundant and their compressed signature matrices are nonsingular. Thus $\mathrm{Pl}-\# \operatorname{CSP}^{2}\left(f^{\prime \prime}\right)$ is \#P-hard by Lemma 9.25 and we are done.

The next lemma shows that if $\partial(f)=[1,0]^{\otimes 2 n-2}+t[0,1]^{\otimes 2 n-2}$ with $t \neq 0$, then either $f=[1,0]^{\otimes 2 n}+t[0,1]^{\otimes 2 n}$ or Pl-\#CSP $(f)$ is \#P-hard. We will use this lemma in Theorem 16.5 for the cases where $\partial(f)$ is a non-degenerate generalized equality GEN-EQ.

For $f=[a, b]^{\otimes 2 n}=\left[f_{0}, f_{1}, \ldots, f_{2 n}\right]$ we have $f_{k}=a^{n-k} b^{k}$. Then it is easy to see that the signature $\bar{f}$ consisting of all even indexed entries of $f$, namely $\bar{f}=$ $\left[f_{0}, f_{2}, \ldots, f_{2 n}\right]$, is just $\left[a^{2}, b^{2}\right]^{\otimes n}$. This observation also extends to a sum of tensor powers by linearity. We will use this simple fact in the next lemma.

Lemma 16.3 Suppose that $(x, y) \neq(0,0)$ and $f=x[1, i]^{\otimes 2 n}+y[1,-i]^{\otimes 2 n}+$ $[1,0]^{\otimes 2 n}+t[0,1]^{\otimes 2 n}$, where $2 n \geq 6$ and $t \neq 0$, then $\mathrm{Pl}$-\#CSP${ }^{2}(f)$ is \#P-hard.

Proof Let $a=x+y, b=(x-y) i$, then $(a, b) \neq(0,0)$. Note that $f=[a, b,-a,-b, \ldots, \pm b, \mp a]+[1,0, \ldots, 0, t]=[a+1, b,-a,-b, a, \ldots, \pm b, \mp a+t]$.

Since $M_{f}$ has a rank 3 submatrix $\left[\begin{array}{lll}f_{0} & f_{1} & f_{2} \\ f_{1} & f_{2} & f_{3} \\ f_{2} & f_{3} & f_{4} \\ f_{2 n-2} & f_{2 n-1} & f_{2 n}\end{array}\right]=\left[\begin{array}{lll}a+1 & b & -a \\ b & -a & -b \\ -a & -b & a \\ \pm a & \pm b \mp a+t\end{array}\right]$, $M_{f}$ has rank 3. By Lemma 16.1, $f$ does not satisfy any second order recurrence relation. So $f \notin \mathscr{P} \cup \widetilde{\mathscr{A}} \cup \widetilde{\mathscr{M}}$ by Lemma 9.11.

1. For $a \neq 0$, let $\bar{f}=\left[f_{0}, f_{2}, \ldots, f_{2 n}\right]$, then $\bar{f}=[a,-a, a,-a, \ldots]+$ $[1,0, \ldots, 0, t]$ is

$$
a[1,-1]^{\otimes n}+[1,0]^{\otimes n}+t[0,1]^{\otimes n},
$$

and $\mathrm{Pl}-\# \operatorname{CSP}(\bar{f}) \leq \operatorname{Pl}-\# \operatorname{CSP}^{2}(f)$ by Lemma 9.19. Note that $\bar{f}=[a+$ $1,-a, a, \ldots, \pm a, \mp a+t]$ has arity $n \geq 3$.

- For $2 n \geq 8$ or [2n=6 and $t \neq-1]$, we claim that $\bar{f} \notin \mathscr{P} \cup \mathscr{A} \cup \widehat{\mathscr{M}}$. 
For $2 n \geq 8$, Since $M_{\bar{f}}$ has a rank 3 submatrix $\left[\begin{array}{ccc}\bar{f}_{0} & \bar{f}_{1} & \bar{f}_{2} \\ \bar{f}_{1} & \bar{f}_{2} & \bar{f}_{3} \\ \bar{f}_{n-2} & \bar{f}_{n-1} & \bar{f}_{n}\end{array}\right]=$ $\left[\begin{array}{lll}a+1 & -a & a \\ -a & a & -a \\ \mp a & \pm a & \mp a+t\end{array}\right], M_{\bar{f}}$ has rank 3 . Thus $\bar{f}$ does not satisfy any secondorder recurrence relation by Lemma 16.1. So $\bar{f} \notin \mathscr{P} \cup \mathscr{A} \cup \widehat{\mathscr{M}}$ by Lemma 9.11.

For $2 n=6$ and $t \neq-1, M_{\bar{f}}$ is a $2 \times 3$ matrix and has rank less than 3 . So it always satisfies a second-order recurrence relation. But we still show that $\bar{f} \notin \mathscr{P} \cup \mathscr{A} \cup \widehat{\mathscr{M}}$.

Note that $\bar{f}=[a+1,-a, a,-a+t]$ when $n=3$.

- $\quad \bar{f}$ is non-degenerate by $\left(\bar{f}_{1}\right)^{2} \neq \bar{f}_{0} \bar{f}_{2}$ and $\bar{f}$ is not GEN-EQ since $\bar{f}_{1} \neq$ 0 , so $\bar{f} \notin \mathscr{P}$.

- If $\bar{f} \in \mathscr{A} \backslash \mathscr{P}$, then $\bar{f}$ has type $\langle 1,0, \pm 1\rangle$ by Lemma 9.11. By $\bar{f}_{0}-$ $\bar{f}_{2} \neq 0, \bar{f}$ does not have type $\langle 1,0,-1\rangle$. If $\bar{f}$ has type $\langle 1,0,1\rangle$, then $\bar{f}_{0}+\bar{f}_{2}=0, \bar{f}_{1}+\bar{f}_{3}=0$. This implies $t=-1$. It is a contradiction. Thus $\bar{f} \notin \mathscr{A} \backslash \mathscr{P}$.

- By $\bar{f}_{1}=-\bar{f}_{2} \neq 0$ and Lemma 9.16, if $\bar{f} \in \widehat{\mathscr{M}}$, then $\bar{f}_{0}=-\bar{f}_{3}$. This contradicts that $t \neq-1$. Thus $\bar{f} \notin \widehat{\mathscr{M}}$.

To summarize, $\bar{f} \notin \mathscr{P} \cup \mathscr{A} \cup \widehat{\mathscr{M}}$ for $2 n \geq 8$, or [ $2 n=6$ and $t \neq-1]$. Thus Pl-\#CSP $(\bar{f})$ is \#P-hard by Theorem 9.22. So Pl-\#CSP $(f)$ is \#P-hard.

- For $2 n=6$ and $t=-1$, we have $f=[a+1, b,-a,-b, a, b,-a-1]$. Firstly, we have $\partial^{2}(f)=[1,0,-1]$ and $f^{\prime}=\partial_{[1,0,-1]}(f)=[1+$ $2 a, 2 b,-2 a,-2 b, 1+2 a]$. The compressed signature matrix of $f^{\prime}$ is $\left[\begin{array}{lll}1+2 a & 2 b & -2 a \\ 2 b & -2 a & -2 b \\ -2 a & -2 b & 1+2 a\end{array}\right]$ and its determinant is $-2\left(4 a^{2}+4 b^{2}+a\right)$. If $4 a^{2}+4 b^{2}+a \neq 0$, then it is nonsingular, and we are done by Lemma 9.25.

Otherwise we have $4 a^{2}+4 b^{2}+a=0$. Consider the gadget in Fig. 35 . We assign $f$ to both vertices. The signature of this gadget is redundant, and its compressed signature matrix is

$$
\left[\begin{array}{ccc}
1+2 a+8 a^{2}+8 b^{2} & b & -2 a-8 a^{2}-8 b^{2} \\
b & 8 a^{2}+8 b^{2} & -b \\
-2 a-8 a^{2}-8 b^{2} & -b & 1+2 a+8 a^{2}+8 b^{2}
\end{array}\right]=\left[\begin{array}{ccc}
1 & b & 0 \\
b & -2 a & -b \\
0 & -b & 1
\end{array}\right] .
$$

Fig. 35 Gadget used to obtain a signature whose signature matrix is redundant. Both vertices are assigned $f$

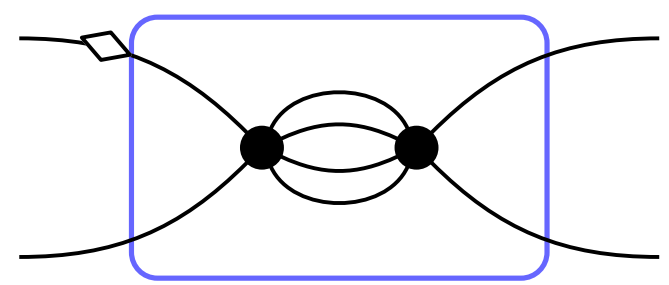


If $a+b^{2} \neq 0$, then this matrix is nonsingular, so we are done by Lemma 9.25.

Otherwise we have $4 a^{2}+4 b^{2}+a=0$ and $a+b^{2}=0$. Also we have $a \neq 0$. By solving these two equations, $a=\frac{3}{4}$ and $b= \pm \frac{\sqrt{3}}{2} i$. Moreover, we have $\partial_{=4}(f)=[1+2 a, 2 b,-1-2 a]=\left[\frac{5}{2}, \pm \sqrt{3} i,-\frac{5}{2}\right]$. By Lemma 9.14, $\partial_{=}(f) \in \widehat{\mathscr{M}}^{\dagger} \backslash(\mathscr{P} \cup \mathscr{A})$. Recall that $f \notin \widehat{\mathscr{M}}$. Thus $\operatorname{Pl}^{\dagger} \# \operatorname{CSP}^{2}(f,[1+$ $2 a, 2 b,-1-2 a])$ is \#P-hard by Lemma 13.7 and we are done.

2. For $a=0$, then $b \neq 0$ by $(a, b) \neq(0,0)$.

- if $2 n \equiv 0(\bmod 4)$ and $t \neq-1$, then

$$
f^{\prime \prime}=\partial_{=4}^{\frac{n-2}{2}}(f)=2^{\frac{n-2}{2}} x[1, i]^{\otimes 4}+2^{\frac{n-2}{2}} y[1,-i]^{\otimes 4}+[1,0]^{\otimes 4}+t[0,1]^{\otimes 4},
$$

i.e., $f^{\prime \prime}=\left[1,2^{\frac{n-2}{2}} b, 0,-2^{\frac{n-2}{2}} b, t\right]$. Note that $f^{\prime \prime}$ is redundant and the determinant of its compressed signature matrix is $-2^{n-2} b^{2}(t+1)$. By $t \neq-1$ and $b \neq 0$, the compressed signature matrix is nonsingular. So $\mathrm{Pl}-\# \mathrm{CSP}^{2}\left(f^{\prime \prime}\right)$ is \#P-hard by Lemma 9.25. Thus $\mathrm{Pl}-\# \mathrm{CSP}^{2}(f)$ is \#P-hard.

- if $2 n \equiv 0(\bmod 4)$ and $t=-1$, we have $\partial^{n-1}(f)=[1,0,-1]$ and

$$
\begin{aligned}
& f^{\prime \prime \prime}=\partial_{[1,0,-1]}^{n-3}(f)=2^{n-3} x[1, i]^{\otimes 6}+2^{n-3} y[1,-i]^{\otimes 6}+[1,0]^{\otimes 6}+(-1)^{n-2}[0,1]^{\otimes 6}, \\
& \text { i.e., } f^{\prime \prime \prime}=\left[1,2^{n-3} b, 0,-2^{n-3} b, 0,2^{n-3} b,(-1)^{n-2}\right] . \text { By Lemma 16.2, } \\
& \mathrm{Pl} \text {-\#CSP }\left(f^{\prime \prime \prime}\right) \text { is \#P-hard and we are done. }
\end{aligned}
$$

- if $2 n \equiv 2(\bmod 4)$, we have

$$
f^{(4)}=\partial_{=4}^{\frac{n-3}{2}}(f)=2^{\frac{n-3}{2}} x[1, i]^{\otimes 6}+2^{\frac{n-3}{2}} y[1,-i]^{\otimes 6}+[1,0]^{\otimes 6}+t[0,1]^{\otimes 6} .
$$

Note that $f^{(4)}=\left[1,2^{\frac{n-3}{2}} b, 0,-2^{\frac{n-3}{2}} b, 0,2^{\frac{n-3}{2}} b, t\right]$. By Lemma 16.2, $\mathrm{Pl}-\# \operatorname{CSP}^{2}\left(f^{(4)}\right)$ is \#P-hard and we are done.

We will use the next lemma in the proof of Theorem 16.5 for the case that $\partial(f)=$ $[1, i]^{\otimes 2 n-2}+i^{r}[1,-i]^{\otimes 2 n-2}$. In this case, we will transform Pl-\#CSP ${ }^{2}$ to Pl-\#CSP 4 by holographic transformation and gadget construction. This is why we have to deal with Pl-\#CSP 4 problems in the next lemma.

Lemma 16.4 Suppose $f=[0,1,0, \ldots, 0, a, 0]$ has arity $2 n \geq 6$. If $a^{4}=1$, then the problem $\mathrm{Pl}-\# \mathrm{CSP}^{4}\left(f,[1,0,1]^{\otimes 2},[1,0,1,0,1]\right)$ is \#P-hard.

Proof In Pl-\#CSP $\left(f,[1,0,1]^{\otimes 2},[1,0,1,0,1]\right)$, we do not have $=2$ on the left, so we cannot connect the two edges on the right freely. But we do have $[1,0,1]^{\otimes 2}$ on the right and $=_{4}$ on the left, so we can do a loop to a pair of $=_{4}$ on the left respectively and we get $[1,0,1]^{\otimes 2}$ on the left.

Suppose $a^{2}=1$. Consider the gadget in Fig. 36a. We assign $f$ to the circle vertices and $[1,0,1]^{\otimes 2}$ to the dashed subgadgets rotated so that it is equivalent to assigning $[1,0,1]$ to the square vertices, where there are $2 n-2$ parallel edges connecting the 2 copies of $f$ with $2 n-2$ square vertices. The signature $f^{\prime}$ of this gadget is 
redundant, and its compressed signature matrix is $\left[\begin{array}{lll}2 n-2 & 0 & 0 \\ 0 & 1+a^{2} & 0 \\ 0 & 0 & (2 n-2) a^{2}\end{array}\right]$, which is nonsingular, by $a^{2}=1$. Thus we have $\mathrm{Pl} \# \operatorname{CSP}^{2}\left(f^{\prime}\right) \leq_{T} \operatorname{Pl}-\# \operatorname{CSP}^{4}\left(f^{\prime},[1,0,1]^{\otimes 2}\right) \leq_{T} \mathrm{Pl}-\# \operatorname{CSP}^{4}\left(f,[1,0,1]^{\otimes 2},[1,0,1,0,1]\right)$, where the first $\leq_{T}$ is by Lemma 10.4. Then we are done by Lemma 9.25.

For $a^{2}=-1$, the gadget in Fig. 36a cannot work since the compressed signature matrix of its resulting signature is $\left[\begin{array}{lll}2 n-2 & 0 & 0 \\ 0 & 0 & 0 \\ 0 & 0 & -2 n+2\end{array}\right]$ which is singular. We consider two cases.

- Suppose $2 n \equiv 0(\bmod 4)$. Then by Lemma 10.4 , we have

$$
\mathrm{Pl}-\# \operatorname{CSP}^{2}(f,[1,0,1,0,1]) \leq_{T} \mathrm{Pl}-\# \operatorname{CSP}^{4}\left(f,[1,0,1,0,1],[1,0,1]^{\otimes 2}\right) .
$$

In $\mathrm{Pl} \# \mathrm{CSP}^{2}(f,[1,0,1,0,1])$, we have $f^{\prime}=\partial^{n-2}(f)=[0,1,0, \pm i, 0]$. Note that $f^{\prime} \in \mathscr{A}^{\dagger}$ by considering $\left[\begin{array}{ll}1 & 0 \\ 0 & \alpha\end{array}\right]^{\otimes 4} f^{\prime}$, and also $f^{\prime} \notin \mathscr{P} \cup \mathscr{A} \cup \widetilde{\mathscr{M}}$ by considering its type $\langle 1,0, \pm i\rangle$, and by Lemma 9.11 . Furthermore we have $[1,0,1,0,1] \in \mathscr{A}$, and also $[1,0,1,0,1] \notin \mathscr{A}^{\dagger}$ by its type $\langle 1,0,-1\rangle$, and by Lemma 9.11. Thus $\mathrm{Pl} \# \mathrm{CSP}^{2}\left(f^{\prime},[1,0,1,0,1]\right)$ is \#P-hard by Theorem 15.4 and we are done.

- For $2 n \equiv 2(\bmod 4)$, we cannot use Lemma 10.4 to get the reduction in (16.28) since Lemma 10.4 requires that all signatures on the right have arity $\equiv 0(\bmod 4)$. But we have $f^{\prime}=\partial_{=4}^{\frac{n-3}{2}}(f)=[0,1,0,0,0, \pm i, 0]$ as well as $\partial_{=4}\left(f^{\prime}\right)=(1 \pm i)[0,1,0]$. We may use $[1,0,1]^{\otimes 2}$ of the LHS to transport this $[0,1,0]$ from the RHS to the LHS as follows: Let $f\left(x_{1}, y_{1}, x_{2}, y_{2}\right)$ be the function $[1,0,1]^{\otimes 2}$ which is 1 iff $x_{1}=y_{1}$ and $x_{2}=y_{2}$, and 0 otherwise. Then we connect $x_{1}$ and $x_{2}$ with the two edges of $[0,1,0]$ from the RHS. This creates $[0,1,0]$ on the LHS, with which we can take derivative of $f^{\prime}$ from the RHS. Then we have $\partial_{[0,1,0]}\left(f^{\prime}\right)=[1,0,0,0, \pm i]$. Consider the gadget in Fig. 36b. We assign $f^{\prime}$ to the circle vertices, $[1,0,0,0, \pm i]$ to the triangle vertex, and $[1,0,1]^{\otimes 2}$ to the dashed subgadgets rotated so that it is equivalent to assigning

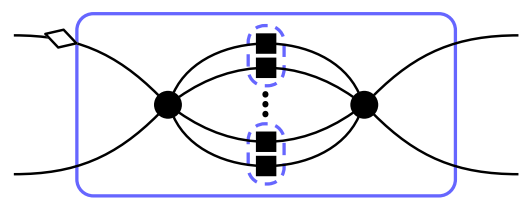

(a)

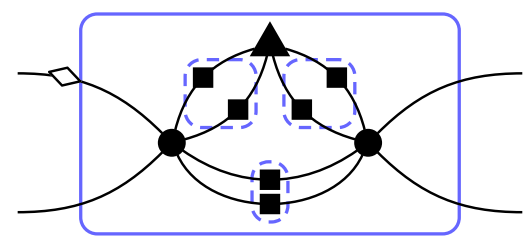

(b)

Fig. 36 Two gadgets used to obtain a signature whose signature matrix is redundant. The dashed subgadgets are assigned $[1,0,1]^{\otimes 2}$ rotated so that it is equivalent to assigning $[1,0,1]$ to the square vertices 
$[1,0,1]$ to the square vertices. The signature $f^{\prime \prime}$ of this gadget is redundant, and its compressed signature matrix is $\left[\begin{array}{lll}2 & 0 & 0 \\ 0 & 1 \mp i & 0 \\ 0 & 0 & \mp 2 i\end{array}\right]$, which is nonsingular. Thus $\mathrm{Pl}$-\#CSP${ }^{2}\left(f^{\prime \prime}\right)$ is \#P-hard by Lemma 9.25. Moreover, we have

$$
\mathrm{Pl}-\# \mathrm{CSP}^{4}\left(f^{\prime \prime},[1,0,1]^{\otimes 2}\right) \leq T \mathrm{Pl}^{2} \# \mathrm{CSP}^{4}\left(f,[1,0,1,0,1],[1,0,1]^{\otimes 2}\right)
$$

and

$$
\mathrm{Pl}-\# \mathrm{CSP}^{2}\left(f^{\prime \prime}\right) \leq_{T} \mathrm{Pl}-\# \mathrm{CSP}^{4}\left(f^{\prime \prime},[1,0,1]^{\otimes 2}\right)
$$

by Lemma 10.4 and we are done. Now Lemma 10.4 can work since $f^{\prime \prime}$ has arity 4.

Now we are ready to prove the main theorem of this section, the dichotomy of $\mathrm{Pl}$-\#CSP${ }^{2}(f)$, where $f$ has a general even arity $2 n$. We will prove the theorem by induction on the arity $2 n$. The base cases $2 n=2$ and $2 n=4$ are already done in Theorem 9.21' and Theorem 12.5, respectively. We always have $f^{\prime}=\partial(f)$ in $\mathrm{Pl}$ \#CSP${ }^{2}(f)$ which has arity $2 n-2$. If $f^{\prime} \notin \mathscr{P} \cup \widetilde{\mathscr{A}} \cup \widetilde{\mathscr{M}}$, then $\mathrm{Pl}-\# \mathrm{CSP}^{2}\left(f^{\prime}\right)$ is \#Phard by induction and $\mathrm{Pl}$-\#CSP${ }^{2}(f)$ is \#P-hard. Otherwise, for $f^{\prime} \in \mathscr{P} \cup \widetilde{\mathscr{A}} \cup \widetilde{\mathscr{M}}$, we can explicitly express $f$ by the integral operator $\int\left(f^{\prime}\right)$. We will prove the theorem in the following order:

(1) $f^{\prime} \in \mathscr{P},(2) f^{\prime} \in \mathscr{A}^{\dagger} \backslash \mathscr{P}$, (3) $f^{\prime} \in \mathscr{A} \backslash \mathscr{P}$, (4) $\left.f^{\prime} \in \widehat{\mathscr{M} \backslash} \backslash \mathscr{P} \cup \mathscr{A}\right)$, and (5) $f^{\prime} \in \widehat{\mathscr{M}^{\dagger}} \backslash(\mathscr{P} \cup \tilde{\mathscr{A}})$.

Note that by Corollary 9.12, Case (2) is equivalent to $f^{\prime} \in \mathscr{A}^{\dagger} \backslash(\mathscr{P} \cup \mathscr{A})$, and Case (3) is equivalent to $f^{\prime} \in \mathscr{A} \backslash\left(\mathscr{P} \cup \mathscr{A}^{\dagger}\right)$. Similarly, by Corollary 9.13, Case (4) is equivalent to $f^{\prime} \in \widehat{\mathscr{M}} \backslash\left(\mathscr{P} \cup \widetilde{\mathscr{A}} \cup \widehat{\mathscr{M}^{\dagger}}\right)$, and Case (5) is equivalent to $f^{\prime} \in \widehat{\mathscr{M}^{\dagger}} \backslash(\mathscr{P} \cup \widetilde{\mathscr{A}} \cup \widehat{\mathscr{M}})$. It is clear that these five Cases (1)-(5) exhaust all possibilities.

In our proof, to use Theorem 12.5 , we often construct arity 4 signatures by our Calculus with binary signatures or $=4$.

Theorem 16.5 Let $f$ be a symmetric signature of even arity $2 n$. If $f \in \mathscr{P} \cup \widetilde{\mathscr{A}} \cup \widetilde{\mathscr{M}}$, then $\mathrm{Pl}-\# \mathrm{CSP}^{2}(f)$ is tractable. Otherwise, $\mathrm{Pl}-\# \mathrm{CSP}^{2}(f)$ is \#P-hard.

Proof If $f \in \mathscr{P} \cup \widetilde{\mathscr{A}} \cup \widetilde{\mathscr{M}}$, then tractability follows from the definition of $\mathscr{P}$ transformability, $\mathscr{A}$-transformability, and $\mathscr{M}$-transformability. Now suppose $f \notin$ $\mathscr{P} \cup \widetilde{\mathscr{A}} \cup \widetilde{\mathscr{M}}$. If $2 n \in\{2,4\}$, then we are done by Theorem 9.21' and Theorem 12.5 respectively.

For $2 n \geq 6$, we will prove the theorem by induction on arity $2 n$. If $f^{\prime}=\partial(f) \notin$ $\mathscr{P} \cup \mathscr{\mathscr { A }} \cup \widetilde{\mathscr{M}}$, then $\mathrm{Pl}$-\#CSP${ }^{2}\left(f_{\sim}^{\prime}\right)$ is \#P-hard by induction. Thus $\mathrm{Pl}$-\#CSP$(f)$ is \#P-hard. Otherwise, $f^{\prime} \in \mathscr{P} \cup \mathscr{A} \cup \widetilde{\mathscr{M}}$.

1. For $f^{\prime} \in \mathscr{P}$, we have $f^{\prime} \equiv 0$ or $f^{\prime}=[a, b]^{\otimes 2 n-2}$ (where $\left.(a, b) \neq(0,0)\right)$ or $f^{\prime}=[1,0]^{\otimes 2 n-2}+t[0,1]^{\otimes 2 n-2}$ with $t \neq 0$ by definition. Note that $2 n-2 \geq 4$.

(a) $f^{\prime} \equiv 0$. Then $f=x[1, i]^{\otimes 2 n}+y[1,-i]^{\otimes 2 n}$ by Proposition 9.7 (the Explicit List for $\left.\int\left(f^{\prime}\right)\right)$. 
If $x=0$ or $y=0$, then $f \in \mathscr{P}$. If $x y \neq 0$ and $x^{4}=y^{4}$, then $f \in \mathscr{A}$. In the following, assume that $x y \neq 0$ and $x^{4} \neq y^{4}$.

- For $2 n \equiv 0(\bmod 4)$, we have $f^{\prime \prime}=\partial_{=4}^{\frac{n-2}{2}}(f)=2^{\frac{n-2}{2}} x[1, i]^{\otimes 4}+$ $2^{\frac{n-2}{2}} y[1,-i]^{\otimes 4}$. By $x y \neq 0, f^{\prime \prime}$ is non-degenerate, and has the unique recurrence type $\langle 1,0,1\rangle$. Therefore $f^{\prime \prime} \notin \mathscr{P} \cup \mathscr{A}^{\dagger} \cup \widetilde{\mathscr{M}}$ by Lemma 9.11. By $x^{4} \neq y^{4}$ it is also not in $\mathscr{A}$. Thus $f^{\prime \prime} \notin \mathscr{P} \cup \mathscr{A} \cup \widetilde{\mathscr{M}}$. Therefore $\mathrm{Pl}$ \#CSP${ }^{2}\left(f^{\prime \prime}\right)$ is \#P-hard by Theorem 12.5. So Pl-\#CSP${ }^{2}(f)$ is \#P-hard.

- For $2 n \equiv 2(\bmod 4)$, we cannot reduce the arity of $f$ to 4 by $={ }_{4}$ directly as in the previous case. We will construct a binary signature that is not $\lambda[1,0,1]$ to reduce the arity of $f$. Firstly, we have $f^{\prime \prime \prime}=\partial_{=4}^{\frac{n-1}{2}}(f)=$ $2^{\frac{n-1}{2}} x[1, i]^{\otimes 2}+2^{\frac{n-1}{2}} y[1,-i]^{\otimes 2}=2^{\frac{n-1}{2}}[a, b,-a]$, where $a=x+$ $y, b=(x-y) i$. We remark that $[a, b,-a]$ can reduce the arity of $f$, but it involves a case analysis of $a$ and $b$. Instead we use $[a, b,-a]$ to construct a simpler binary signature.

Note that $a \neq 0$ by $x^{4} \neq y^{4}$. Then we have $\partial_{[a, b,-a]}(=4$ )$=a[1,0,-1]$ on the left. Thus we have $f^{(4)}=\partial_{[1,0,-1]}^{n-2}(f)=$ $2^{n-2} x[1, i]^{\otimes 4}+2^{n-2} y[1,-i]^{\otimes 4}$. With the same reason as in the previous case, $f^{(4)} \notin \mathscr{P} \cup \widetilde{\mathscr{A}} \cup \widetilde{\mathscr{M}}$ by its type, and by $x y \neq 0, x^{4} \neq y^{4}$. Thus $\mathrm{Pl}-\# \operatorname{CSP}^{2}\left(f^{(4)}\right)$ is \#P-hard by Theorem 12.5. So Pl-\#CSP${ }^{2}(f)$ is \#P-hard.

(b) $f^{\prime}=[a, b]^{\otimes 2 n-2}$ with $a b \neq 0$. If $a^{2}+b^{2} \neq 0$, we have $\partial^{n-2}(f)=$ $\left(a^{2}+b^{2}\right)^{n-2}[a, b]^{\otimes 2}$ and we are done by Lemma 10.3.

Suppose $a^{2}+b^{2}=0$, i.e., $f^{\prime}=[1, \pm i]^{\otimes 2 n-2}$ up to a scalar.

- For $2 n \equiv 0(\bmod 4)$, we have $\partial_{=4}^{\frac{n-2}{2}}\left(f^{\prime}\right)=2^{\frac{n-2}{2}}[1, \pm i]^{\otimes 2}$ and are done by Lemma 10.3 .

- For $2 n \equiv 2(\bmod 4)$, we cannot get $[1, \pm i]^{\otimes 2}$ in $\mathrm{Pl}-\# \mathrm{CSP}^{2}\left(f^{\prime}\right)$ by Remark 7 (note that the arity of $f^{\prime}$ is $\left.2 n-2 \equiv 0(\bmod 4)\right)$. To get $[1, \pm i]^{\otimes 2}$, we need the help of $f$. By Proposition 9.7 (the Explicit List for $\left.\int\left(f^{\prime}\right)\right), f=x[1, i]^{\otimes 2 n}+y[1,-i]^{\otimes 2 n}+g$, where $g$ has arity $2 n$ and $g_{k}=\frac{1}{4}(\epsilon i)^{k}(2 n-2 k)$. If $x=y=0$, then $f \in \widehat{\mathscr{M}}^{\dagger}$. Otherwise, let $u=x+y, v=(x-y) i$, then $(u, v) \neq(0,0)$. We have $\partial_{=4}^{\frac{n-1}{2}}(f)=2^{\frac{n-1}{2}} x[1, i]^{\otimes 2}+2^{\frac{n-1}{2}} y[1,-i]^{\otimes 2}+2^{\frac{n-3}{2}}[1,0,1]$, i.e., $\partial_{=4}^{\frac{n-1}{2}}(f)=2^{\frac{n-3}{2}}[2 u+1,2 v,-2 u+1]$.

If $u \neq 0$, then we have $\partial_{[2 u+1,2 v,-2 u+1]}(=4)=[2 u+1,0,-2 u+1]$ on the left and $\partial_{[2 u+1,0,-2 u+1]}^{n-2}\left(f^{\prime}\right)=(4 u)^{n-2}[1, \pm i]^{\otimes 2}$. Then we are done by Lemma 10.3 .

If $u=0$, then $v \neq 0$ and we have $[1,2 v, 1]$ and $\partial_{[1,2 v, 1]}^{n-2}\left(f^{\prime}\right)=$ $( \pm 4 v i)^{n-2}[1, \pm i]^{\otimes 2}$. Then we are done by Lemma 10.3 again.

(c) $f^{\prime}=[1,0]^{\otimes 2 n-2}$. Then $f=x[1, i]^{\otimes 2 n}+y[1,-i]^{\otimes 2 n}+[1,0]^{\otimes 2 n}$ by Proposition 9.7 (the Explicit List for $\int\left(f^{\prime}\right)$ ). If $x=y=0$, then $f \in \mathscr{P}$. In the 
following, assume that $(x, y) \neq(0,0)$. Let $a=x+y, b=(x-y) i$, then $(a, b) \neq(0,0)$.

We have $\partial^{n-1}(f)=[1,0]^{\otimes 2}$ and $f^{\prime \prime}=\partial_{[1,0]^{\otimes 2}}^{n-2}(f)=x[1, i]^{\otimes 4}+$ $y[1,-i]^{\otimes 4}+[1,0]^{\otimes 4}$, i.e., $f^{\prime \prime}=[1+a, b,-a,-b, a]$. Note that $f^{\prime \prime}$ is redundant. If $a^{2}+b^{2} \neq 0$, then the compressed signature matrix of $f^{\prime \prime}$ is nonsingular and we are done by Lemma 9.25.

Otherwise, we have $a= \pm i b$. We claim that $f^{\prime \prime} \notin \mathscr{P} \cup \tilde{\mathscr{A}} \cup \widetilde{\mathscr{M}}$. Note that $a b \neq 0$ by $(a, b) \neq(0,0)$ and $a= \pm i b$. If $f^{\prime \prime}$ is degenerate, then by $\left(f_{1}^{\prime \prime}\right)^{2}=f_{0}^{\prime \prime} f_{2}^{\prime \prime}$, we have $-a-a^{2}=b^{2}$. This implies that $a=0$. It is a contradiction. Moreover, note that $f^{\prime \prime}=[1+a, \mp i a,-a, \pm i a, a]$ and has type $\langle 0,1, \mp i\rangle$. Since $f^{\prime \prime}$ is non-degenerate and has arity $\geq 3$, the second order recurrence relation $\langle 0,1, \pm i\rangle$ is unique up to a scalar. Thus $f^{\prime \prime} \notin \mathscr{P} \cup$ $\widetilde{\mathscr{A}} \cup \widetilde{\mathscr{M}}$ by Lemma 9.11. So Pl-\#CSP ${ }^{2}\left(f^{\prime \prime}\right)$ is \#P-hard by Theorem 12.5 and we are done.

(d) $f^{\prime}=[0,1]^{\otimes 2 n-2}$. The proof follows from the previous case by a holographic transformation using $\left[\begin{array}{ll}0 & 1 \\ 1 & 0\end{array}\right]$.

(e) $f^{\prime}=[1,0]^{\otimes 2 n-2}+t[0,1]^{\otimes 2 n-2}$ with $t \neq 0$. Then $f=x[1, i]^{\otimes 2 n}+$ $y[1,-i]^{\otimes 2 n}+[1,0]^{\otimes 2 n}+t[0,1]^{\otimes 2 n}$ by Proposition 9.7 (the Explicit List for $\left.\int\left(f^{\prime}\right)\right)$. If $x=y=0$, then $f \in \mathscr{P}$. Otherwise, we have $(x, y) \neq(0,0)$ and we are done by Lemma 16.3.

2. For $f^{\prime} \in \mathscr{A}^{\dagger} \backslash \mathscr{P}$, we have $f^{\prime}=[1, \alpha]^{\otimes 2 n-2}+i^{r}[1,-\alpha]^{\otimes 2 n-2}$ by definition (See Fig. 24). Then $f=x[1, i]^{\otimes 2 n}+y[1,-i]^{\otimes 2 n}+\frac{1}{1+\alpha^{2}}\left\{[1, \alpha]^{\otimes 2 n}+i^{r}[1,-\alpha]^{\otimes 2 n}\right\}$ by Proposition 9.7 (the Explicit List for $\int\left(f^{\prime}\right)$ ). If $x=y=0$, then $f \in \mathscr{A}^{\dagger}$. In the following, assume that $(x, y) \neq(0,0)$.

Note that $f^{\prime}$ has type $\langle 1,0, \pm i\rangle$ up to a scalar. And this second-order recurrence relation is unique up to a scalar. Thus $f^{\prime} \in \mathscr{A}^{\dagger} \backslash(\mathscr{P} \cup \mathscr{A} \cup \widetilde{\mathscr{M}})$ by Lemma 9.11. In the following, we complete the proof by constructing a signature of even arity in $(\mathscr{P} \cup \mathscr{A} \cup \mathscr{\mathscr { M }}) \backslash \mathscr{A}^{\dagger}$ and apply Theorem 15.4, or constructing an arity 4 signature that is not in $\mathscr{P} \cup \widetilde{\mathscr{A}} \cup \mathscr{\mathscr { M }}$ and apply Theorem 12.5 .

Firstly, we have $f^{\prime \prime}=\partial^{n-3}\left(f^{\prime}\right)=\left(1+\alpha^{2}\right)^{n-3}\left\{[1, \alpha]^{\otimes 4}+i^{r}[1,-\alpha]^{\otimes 4}\right\}$. We will discard the nonzero factor that are powers of $1+\alpha^{2}$. If $r \neq 2$, we have $\partial\left(f^{\prime \prime}\right)=\left(1+i^{r}\right)\left[1, \frac{1-i^{r}}{1+i^{r}} \alpha, \alpha^{2}\right]$ and we have $\partial_{\left[1, \frac{1-i^{r}}{1+i^{r}} \alpha, \alpha^{2}\right]}(=4)=\left[1,0, \alpha^{2}\right]$ on the left. For $r=2, \partial\left(f^{\prime \prime}\right)$ is a nonzero multiple of $[0,1,0]$ and we have $\partial_{[0,1,0]}\left(f^{\prime \prime}\right)=2 \alpha\left[1,0, \alpha^{2}\right]$ on the right. Either way, we can take the derivative (for $\left[1,0, \alpha^{2}\right]$ in RHS we connect it via $(=2)$ of LHS to $f$ )

$$
f^{\prime \prime \prime}=\partial_{\left[1,0, \alpha^{2}\right]}^{n-2}(f)=\left(1-\alpha^{2}\right)^{n-2}\left\{x[1, i]^{\otimes 4}+y[1,-i]^{\otimes 4}\right\} .
$$

Note that $\partial_{\left[1,0, \alpha^{2}\right]}\left([1, \pm \alpha]^{2 n}\right)$ is the identically zero signature, since $\alpha^{4}=-1$.

If $x y=0$, or $\left[x y \neq 0\right.$ and $\left.x^{4}=y^{4}\right]$, then $f^{\prime \prime \prime} \in \mathscr{A} \backslash \mathscr{A}^{\dagger}$. So $\mathrm{Pl}$-\#CSP${ }^{2}\left(f^{\prime}, f^{\prime \prime \prime}\right)$ is \#P-hard by Theorem 15.4. Thus Pl-\#CSP${ }^{2}(f)$ is \#P-hard.

Otherwise, $x y \neq 0$ and $x^{4} \neq y^{4}$, so $f^{\prime \prime \prime} \notin \mathscr{P} \cup \mathscr{A} \cup \widetilde{\mathscr{M}}$ (by the same reason as before: first by its type $\langle 1,0,1\rangle$ it could only possibly be in $\mathscr{A}$ among the five classes by Lemma 9.11; but $x^{4} \neq y^{4}$ rules that out 
too). Thus $\mathrm{Pl} \# \mathrm{CSSP}^{2}\left(f^{\prime \prime \prime}\right)$ is \#P-hard by Theorem 12.5. So $\mathrm{Pl} \# \mathrm{CSP}^{2}(f)$ is \#P-hard.

3. For $f^{\prime} \in \mathscr{A} \backslash \mathscr{P}$, we have $f^{\prime}=[1, \rho]^{\otimes 2 n-2}+i^{r}[1,-\rho]^{\otimes 2 n-2}$ by definition (See Fig. 24).

- If $f^{\prime}=[1,1]^{\otimes 2 n-2}+i^{r}[1,-1]^{\otimes 2 n-2}$, then $f=x[1, i]^{\otimes 2 n}+y[1,-i]^{\otimes 2 n}+$ $\frac{1}{2}\left\{[1,1]^{\otimes 2 n}+i^{r}[1,-1]^{\otimes 2 n}\right\}$ by Proposition 9.7 (the Explicit List for $\int\left(f^{\prime}\right)$ ). If $x=y=0$, then $f \in \mathscr{A}$. In the following, assume that $(x, y) \neq(0,0)$.

By a holographic transformation using $H=\left[\begin{array}{ll}1 & 1 \\ 1 & -1\end{array}\right]$, we have

Pl-Holant $\left([1,0,1],[1,0,1,0,1], \ldots \mid \hat{f}^{\prime}, \hat{f}\right) \equiv \operatorname{Pl}-\# \operatorname{CSP}^{2}\left(f^{\prime}, f\right)$,

where $\hat{f}^{\prime}=\left(H^{-1}\right)^{\otimes 2 n-2} f^{\prime}=[1,0]^{2 n-2}+i^{r}[0,1]^{2 n-2}=\left[1,0, \ldots, 0, i^{r}\right]$, $\hat{f}=\left(H^{-1}\right)^{\otimes 2 n} f=x^{\prime}[1,-i]^{\otimes 2 n}+y^{\prime}[1, i]^{\otimes 2 n}+\frac{1}{2}\left\{[1,0]^{\otimes 2 n}+i^{r}[0,1]^{\otimes 2 n}\right\}$, where $x^{\prime}=\frac{(1+i)^{2 n}}{2^{2 n}} x, y^{\prime}=\frac{(1-i)^{2 n}}{2^{2 n}} y$. Note that $\left(x^{\prime}, y^{\prime}\right) \neq(0,0)$.

Since we have $[1,0,1]$ on the left and $\left[1,0, \ldots, 0, i^{r}\right]$ of arity $2 n-2 \geq 4$ on the right in

$$
\text { Pl-Holant }\left([1,0,1],[1,0,1,0,1], \ldots \mid \hat{f}^{\prime}, \hat{f}\right),
$$

we can construct $=2 k$ on the right for $k \geq 1$ in the following way: Firstly, connect four copies of $\left[1,0, \ldots, 0, i^{r}\right]$ by three copies of $[1,0,1]$ in a planar fashion, to form an equality $[1,0, \ldots, 0,1]$ of arity $4(2 n-2)-6=8 n-14$. Then use $4 n-9$ copies of $[1,0,1]$ to form loops on $(=8 n-14)$, and we get $(=4)$. From this, and $(=2)=[1,0,1]$ on the left, we can get all $(=2 k)$ on the right for $k \geq 1$. Then by $=2$ on the left, we can construct all of $=2 k$ on the left. Thus

$$
\operatorname{Pl}-\# \operatorname{CSP}^{2}\left(\hat{f}^{\prime}, \hat{f}\right) \leq \operatorname{Pl-Holant}\left([1,0,1],[1,0,1,0,1], \ldots \mid \hat{f}^{\prime}, \hat{f}\right) .
$$

By Lemma 16.3 $\mathrm{Pl}-\# \mathrm{CSP}^{2}(\hat{f})$ is \#P-hard. Thus $\mathrm{Pl}-\# \mathrm{CSP}^{2}(f)$ is \#P-hard.

- If $f^{\prime}=[1, i]^{\otimes 2 n-2}+i^{r}[1,-i]^{\otimes 2 n-2}$, then $f=x[1, i]^{\otimes 2 n}+y[1,-i]^{\otimes 2 n}+\tilde{f}$, where $\tilde{f}$ has arity $2 n$ and $\tilde{f}_{k}=\frac{1}{4}\left\{i^{k}(2 n-2 k)+i^{r}(-i)^{k}(2 n-2 k)\right\}$ by Proposition 9.7 (the Explicit List for $\int\left(f^{\prime}\right)$ ). Under the holographic transformation by $Z=\left[\begin{array}{ll}1 & 1 \\ i & -i\end{array}\right]$, the expressions are more revealing: $f=$ $Z^{\otimes 2 n}\left[x, 1,0, \ldots, 0, i^{r}, y\right]$, and $f^{\prime}=\partial(f)=Z^{\otimes(2 n-2)}\left[1,0, \ldots, 0, i^{r}\right]$. However, if we apply the holographic transformation $Z$ to $\operatorname{Pl}-\# \operatorname{CSP}^{2}\left(f, f^{\prime}\right)$, we have

Pl-Holant $\left([0,1,0],[1,0,1,0,1], \ldots \mid \widehat{f}, \widehat{f}^{\prime}\right) \equiv \operatorname{Pl-Holant}\left(\mathcal{E} \mathcal{Q}_{2} \mid f, f^{\prime}\right)$, where $\widehat{f}=\left(Z^{-1}\right)^{\otimes 2 n} f=\left[x, 1,0, \ldots, 0, i^{r}, y\right]$, and $\widehat{f}^{\prime}=\left(Z^{-1}\right)^{\otimes 4} f=$ $\left[1,0, \ldots, 0, i^{r}\right]$. Note that now we do not have $=2$ on the left in Pl-Holant $\left([0,1,0],[1,0,1,0,1], \ldots \mid \widehat{f}, \widehat{f}^{\prime}\right)$. This is inconvenient to construct gadget. So, in the following steps we first try to construct $[1,0,-1]^{\otimes 2}$ on the LHS of $\mathrm{Pl}_{-\# \mathrm{CSP}^{2}}(f)$ to get Pl-Holant $\left([1,0,-1]^{\otimes 2} \cup \mathcal{E} \mathcal{Q}_{2} \mid f\right)$. This will be done with the help of Lemma 9.20. Then after the holographic transformation by $Z$, we have $[1,0,-1]^{\otimes 2} Z^{\otimes 2}=4[1,0,1]^{\otimes 2}$ on the left. 
To apply Lemma 9.20, we construct $[1, i]^{\otimes 4}+i^{s}[1,-i]^{\otimes 4}$ in $\mathrm{Pl}$ \# $\operatorname{CSP}^{2}\left(f, f^{\prime}\right)$ for some $0 \leq s \leq 3$ as follows.

- If $2 n \equiv 2(\bmod 4)$, then we have $\partial_{=4}^{\frac{n-3}{2}}\left(f^{\prime}\right)=2^{\frac{n-3}{2}}\left\{[1, i]^{\otimes 4}+\right.$ $\left.i^{r}[1,-i]^{\otimes 4}\right\}$.

- If $2 n \equiv 0(\bmod 4)$, then we have $\partial_{=4}^{\frac{n-2}{2}}\left(f^{\prime}\right)=2^{\frac{n-2}{2}}\left\{[1, i]^{\otimes 2}+\right.$ $\left.i^{r}[1,-i]^{\otimes 2}\right\}=2^{\frac{n-2}{2}}\left[1+i^{r},\left(1-i^{r}\right) i,-\left(1+i^{r}\right)\right]$. This is a nonzero multiple of $[1, \pm 1,-1]$ if $r \neq 0,2$, a nonzero multiple of [1, $0,-1]$ if $r=0$ and a nonzero multiple of $[0,1,0]$ if $r=2$.

If $r \neq 2$, then we have $\partial_{[1, \pm 1,-1]}(=4)=[1,0,-1]$ on the left and

$$
\partial_{[1,0,-1]}^{n-2}\left(f^{\prime}\right)=2^{n-2}\left\{[1, i]^{\otimes 4}+i^{r}[1,-i]^{\otimes 4}\right\} .
$$

If $r=0$, then we have $\partial_{[1,0,-1]}(=4)=[1,0,-1]$ on the left and again

$$
\partial_{[1,0,-1]}^{n-2}\left(f^{\prime}\right)=2^{n-2}\left\{[1, i]^{\otimes 4}+i^{r}[1,-i]^{\otimes 4}\right\} .
$$

If $r=2$, we have $\partial_{[0,1,0]}^{n-2}\left(f^{\prime}\right)=(2 i)^{n-2}\left\{[1, i]^{\otimes 4}+\right.$ $\left.i^{r}(-1)^{n-2}[1,-i]^{\otimes 4}\right\}$.

Thus we have $f^{\prime \prime}=[1, i]^{\otimes 4}+i^{s}[1,-i]^{\otimes 4}$, for some $0 \leq s \leq 3$, in $\mathrm{Pl}-\# \operatorname{CSP}^{2}\left(f, f^{\prime}\right)$. Then by Lemma 9.20 , we have $[1,0,-1]^{\otimes 2}$ on the left, i.e., we have

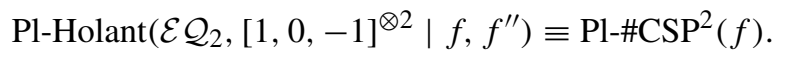

By a holographic transformation using $Z=\left[\begin{array}{ll}1 & 1 \\ i & -i\end{array}\right]$, we have

$$
\begin{aligned}
& \text { Pl-Holant }\left([1,0,1]^{\otimes 2},[0,1,0],[1,0,1,0,1], \ldots \mid \widehat{f}, \widehat{f^{\prime \prime}}\right) \equiv \\
& \text { Pl-Holant }\left(\mathcal{E} \mathcal{Q}_{2},[1,0,-1]^{\otimes 2} \mid f, f^{\prime \prime}\right),
\end{aligned}
$$

where $\widehat{f}=\left(Z^{-1}\right)^{\otimes 2 n} f=\left[x, 1,0, \ldots, 0, i^{r}, y\right]$, and $\widehat{f^{\prime \prime}}=\left(Z^{-1}\right)^{\otimes 4} f=$ $\left[1,0,0,0, i^{s}\right]$.

In Pl-Holant $\left([1,0,1]^{\otimes 2},[0,1,0],[1,0,1,0,1], \ldots \mid \widehat{f}, \widehat{f^{\prime \prime}}\right), \quad$ by $[1,0,1]^{\otimes 2}$ on the left and $\widehat{f^{\prime \prime}}$ on the right, we get $=4$ on the right as follows: Use 4 copies of $\widehat{f^{\prime \prime}}$, connected together by 3 copies of $[1,0,1]^{\otimes 2}$ in a planar way. Each copy of $[1,0,1]^{\otimes 2}$ connects two edges of one copy of $\widehat{f^{\prime \prime}}$ to another copy of $\widehat{f^{\prime \prime}}$ in such a way that the effect is equivalent to connecting them by two copies of $(=2)=[1,0,1]$. This way we get an arity $16-12=4$ signature $(=4)=\left[1,0,0,0,\left(i^{s}\right)^{4}\right]$. Moreover, we have $={ }_{4 k}$ for $k \geq 1$ on the right by $[1,0,1]^{\otimes 2}$ on the left and $=_{4}$ on the right in a similar way. Then we can move $\widehat{f}$ to LHS by $[1,0,1]^{\otimes 2}$ because $\widehat{f}$ has even arity. Thus we have

$$
\begin{aligned}
\text { Pl-Holant }\left([1,0,1]^{\otimes 2},\right. & {\left.[1,0,1,0,1], \widehat{f} \mid \mathcal{E} \mathcal{Q}_{4}\right) \leq \operatorname{Pl}-\operatorname{Holant}\left([1,0,1]^{\otimes 2},\right.} \\
& {\left.[1,0,1,0,1], \ldots \mid \widehat{f}, \widehat{f^{\prime \prime}}\right) . }
\end{aligned}
$$


Note that

$$
\begin{aligned}
& \operatorname{Pl}-\# C^{4}(\widehat{f}, \quad {\left.[1,0,1,0,1],[1,0,1]^{\otimes 2}\right) } \\
& \equiv \operatorname{Pl}-\operatorname{Holant}\left([1,0,1]^{\otimes 2},[1,0,1,0,1], \widehat{f} \mid \mathcal{E} \mathcal{Q}_{4}\right) .
\end{aligned}
$$

We will prove that $\mathrm{Pl}-\# \operatorname{CSP}^{4}\left(\widehat{f},[1,0,1,0,1],[1,0,1]^{\otimes 2}\right)$ is \#P-hard to complete the proof of this case.

Note that $\left[\begin{array}{lll}\widehat{f_{0}} & \widehat{f_{1}} & \widehat{f_{2}} \\ \widehat{f_{1}} & \widehat{f_{2}} & \widehat{f_{3}} \\ \widehat{f_{2 n-3}} & \widehat{f_{2 n-2}} & \widehat{f}_{2 n-1}\end{array}\right]=\left[\begin{array}{lll}x & 1 & 0 \\ 1 & 0 & 0 \\ 0 & 0 & i^{r}\end{array}\right]$ has rank 3. Thus $\widehat{f}$ does not satisfy any second-order recurrence relation by Lemma 16.1. So $\widehat{f} \notin$ $\mathscr{P} \cup \widetilde{\mathscr{A}} \cup \widetilde{\mathscr{M}}$ by Lemma 9.11 .

If $(x, y)=(0,0)$, we are done by Lemma 16.4. In the following, assume that $(x, y) \neq(0,0)$.

$-\quad$ If $2 n \equiv 0(\bmod 4)$, then

$$
\mathrm{Pl}-\# \operatorname{CSP}^{2}(\widehat{f}) \leq \operatorname{Pl}-\# \operatorname{CSP}^{4}\left(\widehat{f},[1,0,1]^{\otimes 2}\right)
$$

by Lemma 10.4 .

For Pl-\#CSP $(\widehat{f})$, we have $\widehat{f^{\prime \prime \prime}}=\partial^{n-2}(\widehat{f})=\left[x, 1,0, i^{r}, y\right]$. Note that $\widehat{f^{\prime \prime \prime}}$ is redundant. If $(-1)^{r} x+y \neq 0$, then the compressed signature matrix of $\widehat{f^{\prime \prime \prime}}$ is nonsingular and we are done by Lemma 9.25.

Otherwise, we have $x= \pm y$, and thus both $x, y \neq 0$. It is easy to see that $\widehat{f^{\prime \prime \prime}}$ does not satisfy the second order recurrence relations $\langle 0,1,0\rangle$, $\langle 1,0, \pm 1\rangle,\langle 1,0, \pm i\rangle$. Thus $\widehat{f^{\prime \prime \prime}} \notin \mathscr{P} \cup \widetilde{\mathscr{A}}$ by Lemma 9.11 .

We consider three possibilities for $\widehat{f^{\prime \prime \prime}}$.

- If $\widehat{f^{\prime \prime \prime}} \in \widehat{\mathscr{M}} \backslash(\mathscr{P} \cup \widetilde{\mathscr{A}})$, then Pl-\#CSP${ }^{2}\left(\widehat{f}, \widehat{f^{\prime \prime \prime}}\right)$ is \#P-hard by Lemma 13.3, where we have $\widehat{f} \notin \widehat{\mathscr{M}}$ because we have noted earlier that $\widehat{f} \notin \mathscr{P} \cup \widetilde{\mathscr{A}} \cup \widetilde{\mathscr{M}}$. Thus $\mathrm{Pl}-\# \mathrm{CSP}^{4}\left(\widehat{f},[1,0,1]^{\otimes 2}\right)$ is \#P-hard by $(16.29)$ and we are done.

- If $\widehat{f^{\prime \prime \prime}} \in \widehat{\mathscr{M}}^{\dagger} \backslash(\mathscr{P} \cup \widetilde{\mathscr{A}})$, then $\widehat{f^{\prime \prime \prime}}=[x, 1,0,1,-x]$ by Corollary 9.18 (the other form $[u, v, w, v, u]$ with $(u+w) w=2 v^{2}$ in Corollary 9.18 is impossible because $w=0$ here and $(u+w) w=2 v^{2}$ would force $v=0$.) Then we are done by Lemma 13.4, because $\widehat{f^{\prime \prime \prime}}$ plays the role of both $f$ and $g$ in Lemma 13.4 , and $\widehat{f} \notin \widehat{\mathscr{M}}^{\dagger}$ by $\widehat{f} \notin \mathscr{P} \cup \widetilde{\mathscr{A}} \cup \widetilde{\mathscr{M}}$.

- If $\widehat{f^{\prime \prime \prime}} \notin \mathscr{P} \cup \widetilde{\mathscr{A}} \cup \widetilde{\mathscr{M}}$, then $\mathrm{Pl}-\# \mathrm{CSP}^{2}\left(\widehat{f^{\prime \prime \prime}}\right)$ is \#P-hard by Theorem 12.5 and we are done.

- For $2 n \equiv 2(\bmod 4)$, we cannot claim the reduction in (16.29) since Lemma 10.4 requires that all signatures on the right have arity $\equiv 0$ $(\bmod 4)$. We get around this difficulty by constructing some arity 4 signatures in $\mathrm{Pl}-\# \operatorname{CSP}^{4}(\widehat{f})$, and then use Lemma 10.4 for these arity 4 signatures.

Firstly, we have $\widehat{g}=\partial_{=4}^{\frac{n-3}{2}}(\hat{f})=\left[x, 1,0,0,0, i^{r}, y\right]$. We also have $\partial_{=}(\widehat{g})=\left[x, 1+i^{r}, y\right]$. They are both on the right. Then we have $\partial_{\left[x, 1+i^{r}, y\right]}(=4)=[x, 0, y]$ on the left. We also connect $[x, 0, y]$ and $\left[x, 1+i^{r}, y\right]$ and then $[x, 0, y]$ in a chain, to get another binary signature 
$h=\left[x^{3},\left(1+i^{r}\right) x y, y^{3}\right]$ on the left. This can be verified by

$$
\left[\begin{array}{ll}
x & 0 \\
0 & y
\end{array}\right]\left[\begin{array}{cc}
x & 1+i^{r} \\
1+i^{r} & y
\end{array}\right]\left[\begin{array}{ll}
x & 0 \\
0 & y
\end{array}\right]=\left[\begin{array}{cc}
x^{3} & \left(1+i^{r}\right) x y \\
\left(1+i^{r}\right) x y & y^{3}
\end{array}\right] .
$$

From these we produce two arity 4 signatures on the right:

$$
\begin{aligned}
& \widehat{g^{\prime}}=\partial_{[x, 0, y]}(\widehat{g})=\left[x^{2}, x, 0, i^{r} y, y^{2}\right] \\
& \widehat{g^{\prime \prime}}=\partial_{h}(\widehat{g})=\left[x^{4}+2\left(1+i^{r}\right) x y, x^{3}, 0, i^{r} y^{3}, y^{4}+2 i^{r}\left(1+i^{r}\right) x y\right] .
\end{aligned}
$$

Thus

$$
\begin{aligned}
& \operatorname{Pl} \# \operatorname{CSP}^{4}\left(\widehat{g^{\prime}}, \widehat{g^{\prime \prime}},[1,0,1]^{\otimes 2},[1,0,1,0,1]\right) \leq \\
& \operatorname{Pl}-\# \operatorname{CSP}^{4}\left(\widehat{f},[1,0,1]^{\otimes 2},[1,0,1,0,1]\right) .
\end{aligned}
$$

Moreover, note that all signatures in $\left.\left\{\widehat{g^{\prime}}, \widehat{g^{\prime \prime}},[1,0,1,0,1]\right)\right\}$ have arity 4 . Then by Lemma 10.4, we have

$\mathrm{Pl}-\# \operatorname{CSP}^{2}\left(\widehat{g^{\prime}}, \widehat{g^{\prime \prime}},[1,0,1,0,1]\right) \leq \mathrm{Pl}-\# \operatorname{CSP}^{4}\left(\widehat{g^{\prime \prime}}, \widehat{g^{\prime \prime}},[1,0,1]^{\otimes 2},[1,0,1,0,1]\right)$.

It is easy to see that $\widehat{g^{\prime}}$ is non-degenerate and does not satisfy the second-order recurrence relations $\langle 0,1,0\rangle,\langle 1,0, \pm 1\rangle,\langle 1,0, \pm i\rangle$, because $(x, y) \neq(0,0)$. Thus $\widehat{g^{\prime}} \notin \mathscr{P} \cup \mathscr{\mathscr { A }}$ by Lemma 9.11. If $\widehat{g^{\prime}} \notin \widetilde{\mathscr{M}}$, then $\mathrm{Pl}-\# \mathrm{CSP}^{2}\left(\widehat{g}^{\prime}\right)$ is \#P-hard by Theorem 12.5 and we are done.

Otherwise, $\widehat{g^{\prime}} \in \widehat{\mathscr{M} \backslash} \backslash(\mathscr{P} \cup \widetilde{\mathscr{A}})$ or $\widehat{g^{\prime}} \in \widehat{\mathscr{M}^{\dagger}} \backslash(\mathscr{P} \cup \widetilde{\mathscr{A}})$.

Note that $[1,0,1,0,1]$ has type $\langle 1,0,-1\rangle$ and the second-order recurrence relation is unique up to a scalar. Thus $[1,0,1,0,1] \notin \widehat{\mathscr{M}}$ by Lemma 9.11. If $\widehat{g^{\prime}} \in \widehat{\mathscr{M} \backslash}(\mathscr{P} \cup \widetilde{\mathscr{A}})$, then $\mathrm{Pl}-\# \operatorname{CSP}^{2}\left(\widehat{g^{\prime}}, \widehat{g^{\prime \prime}},[1,0,1,0,1]\right)$ is \#P-hard by Lemma 13.3 and we are done.

Therefore we may assume $\widehat{g^{\prime}} \in \widehat{\mathscr{M}} \dagger \backslash(\mathscr{P} \cup \widetilde{\mathscr{A}} \cup \widehat{\mathscr{M}})$.

By Corollary 9.18, for $\widehat{g^{\prime}} \in \widehat{\mathscr{M}} \backslash(\mathscr{P} \cup \widetilde{\mathscr{A}} \cup \widehat{\mathscr{M}})$, it cannot be of the form $[u, v, w,-v, u]$ with $(u-w) w=2 v^{2}$; for if it were so, then by $w=0$ in this case, we would have $v=0$, and this would imply that $x=i^{r} y=0$ in $\widehat{g^{\prime}}$. It contradicts that $(x, y) \neq(0,0)$. So $\widehat{g^{\prime}}$ must be of the form $[u, v, 0, v,-u]$, i.e., $x^{2}=-y^{2}, x=i^{r} y$. Thus we have $x=\epsilon i y$ and $i^{r}=\epsilon i$, and $x \neq 0$. Hence both $x, y \neq 0$ and $1+i^{r} \neq 0$. It follows that $x^{3}=-\epsilon i y^{3} \neq \epsilon i y^{3}=i^{r} y^{3}$.

Moreover, if $\widehat{g^{\prime \prime}} \in \widehat{\mathscr{M}^{\dagger}}$, it cannot take the form $[u, v, w,-v, u]$ with $(u-w) w=2 v^{2}$ in Corollary 9.18 because if so then $w=0$ would force $v=0$ and that would force both $x=y=0$. Then $\widehat{g^{\prime \prime}}$ must be of the form $[u, v, 0, v,-u]$. But this would force $x^{3}=i^{r} y^{3}$, a contradiction. Thus $\widehat{g^{\prime \prime}} \notin \widehat{\mathscr{M}^{\dagger}}$.

If $\widehat{g^{\prime \prime}} \notin \mathscr{P} \cup \widetilde{\mathscr{A}} \cup \widetilde{\mathscr{M}}$, then Pl-\#CSP$\left(\widehat{g^{\prime \prime}}\right)$ is \#P-hard by Theorem 12.5 and we are done. Otherwise, $\widehat{g}^{\prime \prime} \in(\mathscr{P} \cup \tilde{\mathscr{A}} \cup \widehat{\mathscr{M}}) \backslash \widehat{\mathscr{M}}$, $\mathrm{Pl}$ \# CSP $\left.\mathrm{CS}^{2}, \widehat{g^{\prime \prime}},[1,0,1,0,1]\right)$ is \#P-hard by Lemma 13.4 and we are done.

4. For $f^{\prime} \in \widehat{\mathscr{M} \backslash} \backslash(\mathscr{P} \cup \tilde{\mathscr{A}})$, we are done by Lemma 13.3 .

5. For $f^{\prime} \in \widehat{\mathscr{M}}^{\dagger} \backslash(\mathscr{P} \cup \mathscr{\mathscr { A }})$, or equivalently by Corollary 9.13, f $\in \widehat{\mathscr{M}}^{\dagger} \backslash(\mathscr{P} \cup$ $\widetilde{\mathscr{A}} \cup \widehat{\mathscr{M})}$. By Lemma 9.14, we have $f^{\prime}=[s, t i]^{\otimes 2 n-2} \pm[t, s i]^{\otimes 2 n-2}$, st $\neq 0$, 
$s^{4} \neq t^{4}$, or $f^{\prime}$ has arity $2 n-2$ and $f_{k}^{\prime}=(\epsilon i)^{k}(2 n-2-2 k)$. Note that we are done if we have a nonzero binary signature that is not $\lambda[1,0,1]$ by Lemma 13.7. Moreover, if we have an arity 4 signature $h$ that is not in $\mathscr{\mathscr { M }}^{\dagger}$ then we are done by the following argument: if $h \in(\mathscr{P} \cup \widetilde{\mathscr{A}} \cup \widehat{\mathscr{M}}) \backslash \widehat{\mathscr{M}} \widehat{\mathscr{A}}^{\dagger}$, then $\mathrm{Pl}-\# \mathrm{CSP}^{2}\left(h, f^{\prime}\right)$ is

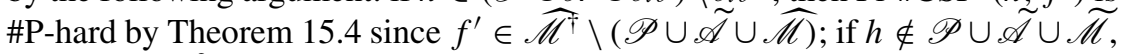
then $\mathrm{Pl}$-\#CSP${ }^{2}(h)$ is \#P-hard by Theorem 12.5 .

In the following, we obtain either a nonzero binary signature that is not $\lambda[1,0,1]$, or a signature of arity 4 not in $\widehat{\mathscr{M}}^{\dagger}$, or we show that $f \in \widehat{\mathscr{M}}^{\dagger}$. The following are an exhaustive list of possibilities of $f^{\prime} \in \widehat{\mathscr{M}}^{\dagger} \backslash(\mathscr{P} \cup \widetilde{\mathscr{A}} \cup \widehat{\mathscr{M}})$, with the case $f^{\prime}=[s, t i]^{\otimes 2 n-2}-[t, s i]^{\otimes 2 n-2}$ with $2 n \equiv 0(\bmod 4)$ being the most resistant, and will be treated last.

- For $f^{\prime}=[s, t i]^{\otimes 2 n-2}+[t, s i]^{\otimes 2 n-2}$ with $2 n \equiv 0(\bmod 4)$ or $f^{\prime}=$ $[s, t i]^{\otimes 2 n-2}-[t, s i]^{\otimes 2 n-2}$ with $2 n \equiv 2(\bmod 4)$, we have $\partial^{n-1}(f)=$ $\left(s^{2}+t^{2}\right)\left(s^{2}-t^{2}\right)^{n-1}\left[1, \frac{2 s t i}{s^{2}+t^{2}},-1\right] \neq \lambda[1,0,1]$.

- For $f^{\prime}=[s, t i]^{\otimes 2 n-2}+[t, s i]^{\otimes 2 n-2}$ with $2 n \equiv 2(\bmod 4), f=$ $x[1, i]^{\otimes 2 n}+y[1,-i]^{\otimes 2 n}+\frac{1}{s^{2}-t^{2}}\left\{[s, t i]^{\otimes 2 n}-[t, s i]^{\otimes 2 n}\right\}$ by Proposition 9.7 (the Explicit List for $\int\left(f^{\prime}\right)$ ). If $x=y=0$, then $f \in \widehat{\mathscr{M}}^{\dagger}$. Otherwise, we have

$$
\begin{aligned}
f^{\prime \prime \prime}=\partial_{=4}^{\frac{n-1}{2}}(f) & =2^{\frac{n-1}{2}} x[1, i]^{\otimes 2}+2^{\frac{n-1}{2}} y[1,-i]^{\otimes 2}+\frac{\left(s^{4}+t^{4}\right)^{\frac{n-1}{2}}}{s^{2}-t^{2}}\left\{[s, t i]^{\otimes 2}-[t, s i]^{\otimes 2}\right\} \\
& =2^{\frac{n-1}{2}} x[1, i]^{\otimes 2}+2^{\frac{n-1}{2}} y[1,-i]^{\otimes 2}+\left(s^{4}+t^{4}\right)^{\frac{n-1}{2}}[1,0,1]
\end{aligned}
$$

Let $a=2^{\frac{n-1}{2}}(x+y), b=2^{\frac{n-1}{2}}(x-y) i$ and $c=\left(s^{4}+t^{4}\right)^{\frac{n-1}{2}}$, then $f^{\prime \prime \prime}=[c+a, b, c-a]$. Note that $(a, b) \neq(0,0)$. If $b \neq 0$, it is obvious that $f^{\prime \prime \prime} \neq \lambda[1,0,1]$. If $b=0$, then $a \neq 0$. Then $f^{\prime \prime \prime} \neq \lambda[1,0,1]$ by $c+a \neq c-a$.

- For the case that $f^{\prime}$ has arity $2 n-2$ and $f_{k}^{\prime}=(\epsilon i)^{k}(2 n-2-2 k)$ with $2 n \equiv 2$ $(\bmod 4)$, we have $f^{\prime \prime}=\partial_{=4}^{\frac{n-3}{2}}\left(f^{\prime}\right)$ which has arity 4 and $f_{k}^{\prime \prime}=2^{\frac{n-3}{2}}(\epsilon i)^{k}(4-$ $2 k)$. Moreover, we have $\partial\left(f^{\prime \prime}\right)=2^{\frac{n+1}{2}}[1, \epsilon i,-1] \neq \lambda[1,0,1]$. We remark that it is necessary to use $={ }_{4}$ that many times, since $f$ with two loops by $=2$ is already identically zero.

- For the case that $f^{\prime}$ has arity $2 n-2$ and $f_{k}^{\prime}=(\epsilon i)^{k}(2 n-2-2 k)$ with $2 n \equiv 0(\bmod 4)$, we may consider only the case where the $\operatorname{sign} \epsilon$ is + . Indeed under $Z=\left[\begin{array}{ll}1 & 1 \\ i & -i\end{array}\right]$, for the $+\operatorname{sign} f^{\prime}=Z^{\otimes(2 n-2)}[0,1,0, \ldots, 0]$ and for the $-\operatorname{sign} f^{\prime}=Z^{\otimes(2 n-2)}[0, \ldots, 0,1,0]$, a reversal under the $Z$ transformation. If we take a holographic transformation by $T=\left[\begin{array}{ll}1 & 0 \\ 0 & -1\end{array}\right]$, we have $T Z=\left[\begin{array}{ll}1 & 1 \\ -i & i\end{array}\right]=Z\left[\begin{array}{ll}0 & 1 \\ 1 & 0\end{array}\right]$, and so $(T Z)^{\otimes(2 n-2)}[0, \ldots, 0,1,0]=$ $Z^{\otimes(2 n-2)}[0,1,0, \ldots, 0]$. Meanwhile, $\mathcal{E} \mathcal{Q}_{2}$ is invariant under $T$.

Thus we consider $f^{\prime}$ of arity $2 n-2$ where $f_{k}^{\prime}=i^{k}(2 n-2-2 k)$ with $2 n \equiv 0(\bmod 4)$. Let $\hat{f}^{\prime}=\left(Z^{-1}\right)^{\otimes(2 n-2)} f^{\prime}=[0,1,0, \ldots, 0]$ and 
let $\hat{f}=\left(Z^{-1}\right)^{\otimes(2 n-2)} f$. Then we have $\left(Z^{-1}\right)^{\otimes(2 n-2)}(\partial(f))=\partial_{[0,1,0]}(\hat{f})$ up to a scalar. This implies $\partial_{[0,1,0]}(\hat{f})=[0,1,0, \ldots, 0]$. Thus there exist constants $x$ and $y$ such that $\hat{f}=[x, 0,1,0, \ldots, 0, y]$. By the holographic transformation using $Z$, we have

$$
\mathrm{Pl}-\# \mathrm{CSP}^{2}(f) \equiv \mathrm{Pl-Holant}([0,1,0],[1,0,1,0,1], \ldots \mid \hat{f}) .
$$

We remark that, in Pl-Holant $([0,1,0],[1,0,1,0,1], \ldots \mid \hat{f})$, all signatures have even arities. And all signatures of arity $2 m \equiv 2(\bmod 4)$ satisfy the odd parity constraints and all signatures of arity $2 m \equiv 0 \bmod 4$ satisfy the even parity constraints. Then by the statement of Remark 6 , any binary signature constructed in Pl-Holant $([0,1,0],[1,0,1,0,1], \ldots \mid \hat{f})$ can only be of the form $\lambda[0,1,0]$. This implies that the binary signature constructed in $\mathrm{Pl}$-\#CSP${ }^{2}(f)$ can only be of the form $\lambda[1,0,1]$ before the $Z$-transformation. This forces us to construct signatures of arity at least 4 to prove hardness.

In Pl-Holant([0, 1, 0], $[1,0,1,0,1], \ldots \mid \hat{f})$, note that by $2 n \equiv 0 \bmod 4$ we have $2 n \geq 8$, and $\hat{g}=\partial_{[1,0,1,0,1]}^{\frac{n-2}{2}}(\hat{f})=\left[x+\frac{n-2}{2} \cdot 6,0,1,0, y\right]$. It has arity 4. If $\left(x+\frac{n-2}{2} \cdot 6\right) y \neq 1$, then $\hat{g} \notin \mathscr{M}$, because symmetric matchgate signatures must form geometric series in alternate terms. Thus we have $Z^{\otimes 4}(\hat{g}) \notin \widehat{\mathscr{M}^{\dagger}}$ in $\operatorname{Pl}_{-\# C^{2}}\left(f, f^{\prime}\right)$ and we are done.

If $\left(x+\frac{n-2}{2} \cdot 6\right) y=1$, then $y \neq 0$. Firstly, we have an arity 8 signature

$$
\widehat{g^{\prime}}=\partial_{[1,0,1,0,1]}^{\frac{n-4}{2}}(\hat{f})=\left[x+\frac{n-4}{2} \cdot 6,0,1,0,0,0,0,0, y\right]
$$

(note that $n \geq 4$ when $2 n \equiv 0 \bmod 4$ ), and we have $\partial_{[0,1,0]}^{2}\left(\widehat{g^{\prime}}\right)=$ $[1,0]^{\otimes 4}$ on the right. So we have $[0,1]^{\otimes 4}$ on the left. Moreover, we have $\partial_{[0,1]^{\otimes 4}}\left(\widehat{g^{\prime}}\right)=y[0,1]^{\otimes 4}$ on the right. So we have $[1,0]^{\otimes 4}$ on the left. Then we have $\widehat{g^{\prime \prime}}=\partial_{[1,0]}^{\frac{n-2}{2}}(\hat{f})=[x, 0,1,0,0]$ on the right. Note that $\widehat{g^{\prime \prime}} \notin \mathscr{M}$. Thus we have $Z^{\otimes 4}\left(\widehat{g^{\prime \prime}}\right) \notin \widehat{\mathscr{M}^{\dagger}}$ in $\mathrm{Pl}-\# \operatorname{CSP}^{2}\left(f, f^{\prime}\right)$ and we are done.

- For the last case of Case $5, f^{\prime}=[s, t i]^{\otimes 2 n-2}-[t, s i]^{\otimes 2 n-2}$ with $2 n \equiv 0$ $(\bmod 4)$, we let $u=\frac{s-t}{s+t}$, then $u^{4} \neq 0,1$ by Lemma 9.3. Let $Z=\left[\begin{array}{ll}1 & 1 \\ i & -i\end{array}\right]$, then

$$
\begin{aligned}
\widehat{f^{\prime}} & =\left(Z^{-1}\right)^{\otimes 2 n-2}\left(f^{\prime}\right) \\
& =\frac{1}{2^{2 n-2}}\left\{[s+t, s-t]^{\otimes 2 n-2}-[s+t, t-s]^{\otimes 2 n-2}\right\} \\
& =\frac{(s+t)^{2 n-2}}{2^{2 n-2}}\left\{[1, u]^{\otimes 2 n-2}-[1,-u]^{\otimes 2 n-2}\right\} \\
& =\lambda\left[0, u^{2}, 0, u^{4}, \ldots, u^{2 n-2}, 0\right],
\end{aligned}
$$

where $\lambda=\frac{(s+t)^{2 n-2}}{2^{2 n-3} u} \neq 0$. Let $\left(Z^{-1}\right)^{\otimes 2 n} f=\widehat{f}$, then $\left(Z^{-1}\right)^{\otimes 2 n-2}$ $(\partial(f))=\partial_{[0,1,0]}(\widehat{f})$ up to a scalar. This implies that $\partial_{[0,1,0]}(\widehat{f})=$ $\lambda\left[0, u^{2}, 0, u^{4}, \ldots, u^{2 n-2}, 0\right]$. Thus there exist constants $x$ and $y$ such that $\widehat{f}=\left(Z^{-1}\right)^{\otimes 2 n} f=\lambda\left[1+x, 0, u^{2}, 0, u^{4}, \ldots, u^{2 n-2}, 0, u^{2 n}+y\right]$, where we 
append the terms 1 and $u^{2 n}$ for future convenience. (This can be accommodated by naming different $x$ and $y$.) If $x=y=0$, then $\widehat{f} \in \mathscr{M}$ and $f \in \widehat{\mathscr{M}}^{\dagger}$. In the following, assume that $(x, y) \neq(0,0)$. By the holographic transformation using $Z$, we have

$$
\mathrm{Pl}-\# \mathrm{CSP}^{2}(f) \equiv \mathrm{Pl-Holant}([0,1,0],[1,0,1,0,1], \ldots \mid \widehat{f}) .
$$

By the same argument as the previous case, it is impossible to construct a "good" binary signature in this case. So we have to construct signatures of arity at least 4 to prove hardness.

We will repeatedly use the following computation in the remainder of this proof: Let $\bar{g}=\partial_{\left[1,0, v, 0, v^{2}\right]}(g)$ for some $v$, then $\operatorname{arity}(\bar{g})=\operatorname{arity}(g)-4$ and $\bar{g}_{k}=g_{k}+6 v g_{k+2}+v^{2} g_{k+4}$.

We will complete the proof by constructing some arity 4 signatures $\widehat{h}$ in the setting after the $Z$-transformation Pl-Holant $([0,1,0],[1,0,1,0,1], \ldots$ | $\widehat{f})$ that cannot all belong to $\mathscr{M}$. We note that if $\widehat{h} \notin \mathscr{M}$ then $h=Z^{\otimes 4} \widehat{h} \notin$ $\widehat{\mathscr{M}^{\dagger}}$. This will imply $\mathrm{Pl}$-\#CSP${ }^{2}\left(h, f^{\prime}\right)$ is \#P-hard as noted earlier, thus complete the proof of this Case 5 .

In Pl-Holant $([0,1,0],[1,0,1,0,1], \ldots \mid \widehat{f})$, we have $\partial_{[0,1,0]}^{n-2}(\widehat{f})$ which is a nonzero multiple of $\left[1,0, u^{2}, 0, u^{4}\right]$. Then we have $\left[u^{4}, 0, u^{2}, 0,1\right]=$ $u^{4}\left[1,0, u^{-2}, 0, u^{-4}\right]$ on the left. Ignoring $\lambda \neq 0$, we write

$$
\widehat{f}=\left[1,0, u^{2}, 0, u^{4}, 0, \ldots, 0, u^{2 n}\right]+[x, 0,0,0,0, \ldots, 0, y]
$$

which has arity $2 n \geq 8$, and we have

$$
\begin{aligned}
\widehat{f^{(4)}} & =\partial_{\left[1,0, u^{-2}, 0, u^{-4}\right]}^{\frac{n-4}{2}}(\widehat{f}) \\
& =8^{\frac{n-4}{2}}\left[1,0, u^{2}, 0, u^{4}, 0, u^{6}, 0, u^{8}\right]+\left[x, 0,0,0,0,0,0,0, y u^{-2(n-4)}\right] \\
& =\left[x+8^{\frac{n-4}{2}}, 0,8^{\frac{n-4}{2}} u^{2}, 0,8^{\frac{n-4}{2}} u^{4}, 0,8^{\frac{n-4}{2}} u^{6}, 0,8^{\frac{n-4}{2}} u^{8}+y u^{-2(n-4)}\right] .
\end{aligned}
$$

Let $x^{\prime}=\frac{x}{8^{\frac{n-4}{2}}}, y^{\prime}=\frac{y u^{-2(n-4)}}{8^{\frac{n-4}{2}}}(\operatorname{since}(x, y) \neq(0,0)$ and $u \neq 0$, we have $\left.\left(x^{\prime}, y^{\prime}\right) \neq(0,0)\right)$, then $\widehat{f^{(4)}}=\left[x^{\prime}+1,0, u^{2}, 0, u^{4}, 0, u^{6}, 0, u^{8}+y^{\prime}\right]$ up to the scalar $8^{\frac{n-4}{2}}$. Further, we have $\widehat{f^{(5)}}=\partial_{\left[1,0, u^{-2}, 0, u^{-4}\right]}\left(\widehat{f^{(4)}}\right)=\left[x^{\prime}+\right.$ $\left.8,0,8 u^{2}, 0,8 u^{4}+y^{\prime} u^{-4}\right]$. If $x^{\prime}=0$ or $y^{\prime}=0$, then exactly one of them is zero, and thus $\widehat{f^{(5)}} \notin \mathscr{M}$ by $\left(x^{\prime}+8\right)\left(8 u^{4}+y^{\prime} u^{-4}\right) \neq\left(8 u^{2}\right)^{2}$ and we are done. So we can assume that $x^{\prime} y^{\prime} \neq 0$ in the following.

In the following, if we have a signature $\left[1,0, v, 0, v^{2}\right]$ with $v \neq 0$ on the left, then we have $\partial_{\left[1,0, v, 0, v^{2}\right]}\left(\widehat{f^{(4)}}\right)=\left[x^{\prime}+c, 0, c u^{2}, 0, y^{\prime} v^{2}+c u^{4}\right]$, where $c=1+6 u^{2} v+u^{4} v^{2}$. If $c=0$, then we have $\left[x^{\prime}, 0,0,0, y^{\prime} v^{2}\right] \notin \mathscr{M}$ and we are done. So in the following, we always suppose that $c=1+6 u^{2} v+u^{4} v^{2} \neq$ 0 . Moreover, if $\left(x^{\prime}+c\right)\left(y^{\prime} v^{2}+c u^{4}\right) \neq\left(c u^{2}\right)^{2}$, then $\left[x^{\prime}+c, 0, c u^{2}, 0, y^{\prime} v^{2}+\right.$ $\left.c u^{4}\right] \notin \mathscr{M}$ and we are done. So we assume that $\left(x^{\prime}+c\right)\left(y^{\prime} v^{2}+c u^{4}\right)=$ $\left(c u^{2}\right)^{2}$. This implies that $x^{\prime}+c \neq 0$ and, by expanding this equation the term $c^{2} u^{4}$ cancel and we get $x^{\prime} y^{\prime} v^{2}+\left(x^{\prime} u^{4}+y^{\prime} v^{2}\right) c=0$. To summarize, in the 
following if we have $\left[1,0, v, 0, v^{2}\right]$ with $v \neq 0$ on the left, then we have

$$
\begin{aligned}
& c=1+6 u^{2} v+u^{4} v^{2} \neq 0, \\
& x^{\prime}+c \neq 0, \\
& x^{\prime} y^{\prime} v^{2}+\left(x^{\prime} u^{4}+y^{\prime} v^{2}\right) c=0 .
\end{aligned}
$$

Firstly, by $\widehat{f^{(5)}}=\partial_{\left[1,0, u^{-2}, 0, u^{-4}\right]}\left(\widehat{f^{(4)}}\right)=\left[x^{\prime}+8,0,8 u^{2}, 0,8 u^{4}+y^{\prime} u^{-4}\right]$ and (16.30) (in this case $c$ evaluates to 8), we have

$$
\begin{aligned}
& x^{\prime}+8 \neq 0, \\
& x^{\prime} y^{\prime} u^{-4}+8\left(x^{\prime} u^{4}+y^{\prime} u^{-4}\right)=0 .
\end{aligned}
$$

Note that we have $[1,0,1,0,1]$ on the left, so we have $\widehat{f^{(6)}}=$ $\partial_{[1,0,1,0,1]}\left(\widehat{f^{(4)}}\right)=\left[x^{\prime}+c_{1}, 0, c_{1} u^{2}, 0, y^{\prime}+c_{1} u^{4}\right]$, where $c_{1}=1+6 u^{2}+u^{4}$. Then by (16.30), we have $c_{1} \neq 0$ and

$$
\begin{aligned}
& x^{\prime}+c_{1} \neq 0, \\
& x^{\prime} y^{\prime}+\left(x^{\prime} u^{4}+y^{\prime}\right) c_{1}=0 .
\end{aligned}
$$

By (16.31), (16.32), and $x^{\prime} y^{\prime} \neq 0$, we have

$$
\begin{aligned}
& 1+\left(\frac{u^{8}}{y^{\prime}}+\frac{1}{x^{\prime}}\right) 8=0 \\
& 1+\left(\frac{u^{4}}{y^{\prime}}+\frac{1}{x^{\prime}}\right) c_{1}=0 .
\end{aligned}
$$

Then we have

$$
\begin{aligned}
& \frac{1}{x^{\prime}}=\frac{c_{1}-8 u^{4}}{8 c_{1}\left(u^{4}-1\right)}=-\frac{7 u^{2}+1}{8\left(u^{2}+1\right)\left(u^{4}+6 u^{2}+1\right)} \\
& \frac{1}{y^{\prime}}=\frac{8-c_{1}}{8 c_{1}\left(u^{8}-u^{4}\right)}=-\frac{u^{2}+7}{8 u^{4}\left(u^{2}+1\right)\left(u^{4}+6 u^{2}+1\right)}
\end{aligned}
$$

Since $1 / x^{\prime} \neq 0$, we have $7 u^{2}+1 \neq 0$.

For $\widehat{f^{(5)}}, \widehat{f^{(6)}}$, let $v_{2}=\frac{x^{\prime}+8}{8 u^{2}}$ and $v_{3}=\frac{x^{\prime}+c_{1}}{c_{1} u^{2}}$, then $v_{2} \neq 0, v_{3} \neq 0$ by $x^{\prime}+8 \neq 0$ and $x^{\prime}+c_{1} \neq 0$, and $\widehat{f^{(5)}}=\left[1,0, v_{2}^{-1}, 0, v_{2}^{-2}\right], \widehat{f^{(6)}}=$ $\left[1,0, v_{3}^{-1}, 0, v_{3}^{-2}\right]$ up to the scalars $x^{\prime}+8, x^{\prime}+c_{1}$ respectively. So we have $\left[1,0, v_{2}, 0, v_{2}^{2}\right],\left[1,0, v_{3}, 0, v_{3}^{2}\right]$ on the left. Moreover, let $c_{2}=1+6 u^{2} v_{2}+$ $u^{4} v_{2}^{2}, c_{3}=1+6 u^{2} v_{3}+u^{4} v_{3}^{2}$, then we have by (16.30)

$$
\begin{aligned}
& x^{\prime} y^{\prime} v_{2}^{2}+\left(x^{\prime} u^{4}+y^{\prime} v_{2}^{2}\right) c_{2}=0 \\
& x^{\prime} y^{\prime} v_{3}^{2}+\left(x^{\prime} u^{4}+y^{\prime} v_{3}^{2}\right) c_{3}=0 .
\end{aligned}
$$


In (16.33), we have

$$
\begin{aligned}
c_{1} & =u^{4}+6 u^{2}+1, \\
\frac{1}{x^{\prime}} & =\frac{c_{1}-8 u^{4}}{8 c_{1}\left(u^{4}-1\right)}=-\frac{7 u^{2}+1}{8\left(u^{2}+1\right)\left(u^{4}+6 u^{2}+1\right)}, \\
\frac{1}{y^{\prime}} & =\frac{8-c_{1}}{8 c_{1}\left(u^{8}-u^{4}\right)}=-\frac{u^{2}+7}{8 u^{4}\left(u^{2}+1\right)\left(u^{4}+6 u^{2}+1\right)}, \\
v_{2} & =\frac{x^{\prime}+8}{8 u^{2}}=\frac{-u^{4}-7 u^{2}}{7 u^{2}+1}, \\
c_{2} & =1+6 u^{2} v_{2}+u^{4} v_{2}^{2}=\frac{u^{12}+14 u^{10}+7 u^{8}-300 u^{6}+7 u^{4}+14 u^{2}+1}{\left(7 u^{2}+1\right)^{2}}, \\
v_{3} & =\frac{x^{\prime}+c_{1}}{c_{1} u^{2}}=-\frac{u^{2}+7}{u^{2}\left(7 u^{2}+1\right)}, \\
c_{3} & =1+6 u^{2} v_{3}+u^{4} v_{3}^{2}=\frac{8 u^{4}-272 u^{2}+8}{\left(7 u^{2}+1\right)^{2}} .
\end{aligned}
$$

Note that all of them are functions of $u$. Thus (16.33) gives the following two equations of $u$ :

$$
\begin{aligned}
\frac{8 u^{4} c_{1}^{2}\left(u^{2}+1\right)^{2} \cdot p_{1}(u)}{\left(7 u^{2}+1\right)^{4}} & =0, \\
\frac{3072 u^{2}\left(u^{2}+1\right)^{2} c_{1} \cdot p_{2}(u)}{\left(7 u^{2}+1\right)^{4}} & =0,
\end{aligned}
$$

where $p_{1}(u)=u^{12}+14 u^{10}-49 u^{8}-700 u^{6}-49 u^{4}+14 u^{2}+1, p_{2}(u)=$ $7 u^{4}+2 u^{2}+7$. Note that $q_{1}(u) p_{1}(u)+q_{2}(u) p_{2}(u)=244224$, where $q_{1}(u)=$ $-188-315 u^{2}, q_{2}(u)=34916-9555 u^{2}-32872 u^{4}-2058 u^{6}+644 u^{8}+$ $45 u^{10}$, thus $\operatorname{gcd}\left(p_{1}(u), p_{2}(u)\right)=1$. So $p_{1}(u)$ and $p_{2}(u)$ have no common zeros. Then by $u^{4} \neq 0,1, c_{1} \neq 0$ (so the factors other than $p_{1}(u)$ and $p_{2}(u)$ in the numerators of (16.34) are nonzero), the two equations in (16.33) have no common solution in $u$. This is a contradiction and we have finished the proof.

We hereby finish the proof of Theorem 16.5, and hence we complete the proof of the main theorem of Part II-Theorem 9.2 is a straightforward combination of Theorem 11.13, Theorem 16.5 and Theorem 15.4.

Acknowledgements We wish to express our enormous gratitude to the three anonymous referees, who went through the incredibly labor-intensive verification of all the proof in this long paper. Their 20 plus pages of detailed and thoughtful comments were extremely helpful. We benefited greatly from their suggestions and ideas. This resulted in the proofs of several lemmas being rewritten with a clearer presentation. We also thank the editors of the journal for their insight and patience who guided us through the careful revision process.

Open Access This article is licensed under a Creative Commons Attribution 4.0 International License, which permits use, sharing, adaptation, distribution and reproduction in any medium or format, as long as 
you give appropriate credit to the original author(s) and the source, provide a link to the Creative Commons licence, and indicate if changes were made. The images or other third party material in this article are included in the article's Creative Commons licence, unless indicated otherwise in a credit line to the material. If material is not included in the article's Creative Commons licence and your intended use is not permitted by statutory regulation or exceeds the permitted use, you will need to obtain permission directly from the copyright holder. To view a copy of this licence, visit http://creativecommonshorg/licenses/by/4. $0 /$.

\section{References}

1. Backens, M.: A new holant dichotomy inspired by quantum computation. In: ICALP, vol. 80 of LIPIcs, pp. 16:1-16:14. Schloss Dagstuhl - Leibniz-Zentrum fuer Informatik (2017)

2. Backens, M.: A complete dichotomy for complex-valued holant ${ }^{c}$. In: ICALP, vol. 107 of LIPIcs, pp. 12:1-12:14. Schloss Dagstuhl - Leibniz-Zentrum für Informatik (2018)

3. Baxter, R.J.: Exactly Solved Models in Statistical Mechanics. Academic Press, London (1982)

4. Cai, J.-Y., Chen, X.: Complexity dichotomies for counting problems. Cambridge University Press (2018)

5. Cai, J.-Y., Chen, X., Lipton, R.J., Lu, P.: On Tractable exponential sums. In: FAW, pp. 148-159. Springer, Berlin (2010)

6. Cai, J.-Y., Choudhary, V.: Some results on matchgates and holographic algorithms. Int. J Softw. Inform. 1(1), 3-36 (2007)

7. Cai, J.-Y., Choudhary, V., Lu, P.: On the theory of matchgate computations. Theory Comput. Syst. 45(1), 108-132 (2009)

8. Cai, J.-Y., Fu, Z.: Holographic algorithm with matchgates is universal for planar \#CSP over boolean domain. In: STOC, pp. 842-855, ACM, 2017, Journal version to appear in SIAM J. Comput., available at https://doi.org/10.1137/17M1131672

9. Cai, J.-Y., Fu, Z., Guo, H., Williams, T.: A Holant dichotomy: Is the FKT algorithm universal? In: FOCS, pp. 1259-1276. IEEE Computer Society (2015)

10. Cai, J.-Y., Gorenstein, A.: Matchgates revisited. Theory Comput. 10(7), 167-197 (2014)

11. Cai, J.-Y., Guo, H., Williams, T.: A complete dichotomy rises from the capture of vanishing signatures. SIAM J. Comput. 45(5), 1671-1728 (2016)

12. Cai, J.-Y., Guo, H., Williams, T.: Holographic algorithms beyond matchgates. Inf. Comput. 259(1), 102-129 (2018). Preliminary version appeared in ICALP 2014

13. Cai, J.-Y., Kowalczyk, M.: Spin systems on $k$-regular graphs with complex edge functions. Theor Comput. Sci. 461, 2-16 (2012)

14. Cai, J.-Y., Kowalczyk, M., Williams, T.: Gadgets and anti-gadgets leading to a complexity dichotomy. ACM Trans. Comput. Theory 11(2), 7:1-7:26 (2019). Preliminary version appeared in ITCS 2012

15. Cai, J.-Y., Lu, P.: On symmetric signatures in holographic algorithms. Theory Comput. Syst. 46(3), 398-415 (2010)

16. Cai, J.-Y., Lu, P.: Holographic algorithms: from art to science. J. Comput. Syst. Sci. 77(1), 41-61 (2011)

17. Cai, J.-Y., Lu, P., Xia, M.: Computational complexity of Holant problems. SIAM J. Comput. 40(4), 1101-1132 (2011)

18. Cai, J.-Y., Lu, P., Xia, M.: The complexity of complex weighted Boolean \#CSP. J. Comput. System Sci. 80(1), 217-236 (2014)

19. Cai, J.-Y., Lu, P., Xia, M.: Holographic algorithms with matchgates capture precisely tractable planar \#CSP. SIAM J. Comput. 46(3), 853-889 (2017)

20. Cai, J.-Y., Lu, P., Xia, M.: Dichotomy for Real Holant ${ }^{c}$ Problems. In: SODA, pp. 1802-1821. SIAM (2018)

21. Cai, J.-Y., Lu, P., Xia, M.: Dichotomy for Holant* problems on the Boolean domain. Theory Comput. Syst. 64(8), 1362-1391 (2020). Preliminary version appeared in SODA 2011

22. Dodson, C.T.J., Poston, T.: Tensor Geometry. Graduate Texts in Mathematics. Springer, 2nd edn (1991)

23. Draisma, J., Gijswijt, D.C., Lovász, L., Regts, G., Schrijver, A.: Characterizing partition functions of the vertex model. J. Algebra. 350, 197-206 (2012) 
24. Freedman, M., Lovász, L., Schrijver, A.: Reflection positivity, rank connectivity, and homomorphism of graphs. J. Amer. Math. Soc. 20(1), 37-51 (2007)

25. Guo, H., Lu, P., Valiant, L.G.: The complexity of symmetric Boolean parity Holant problems. SIAM J. Comput. 42(1), 324-356 (2013)

26. Guo, H., Williams, T.: The complexity of planar Boolean \#CSP with complex weights. J. Comput. Syst. Sci. 107, 1-27 (2020). Preliminary version appeared inn ICALP 2013

27. Huang, S., Lu, P.: A dichotomy for real weighted Holant problems. Comput. Complex. 25(1), 255-304 (2016)

28. Ising, E.: Beitrag zür Theorie des Ferromagnetismus. Zeitschrift für Physik 31(1), 253-258 (1925)

29. Kasteleyn, P.W.: The statistics of dimers on a lattice. Physica 27, 1209-1225 (1961)

30. Kasteleyn, P.W.: Graph theory and crystal physics. In: Graph Theory and Theoretical Physics, pp. 43110. Academic Press, London (1967)

31. Kowalczyk, M.: Dichotomy theorems for Holant problems. PhD thesis, University of WisconsinMadison. Available at http://cs.nmu.edu/ mkowalcz/research/main.pdf (2010)

32. Landsberg, J.M., Morton, J., Norine, S.: Holographic algorithms without matchgates. Linear Algebra Appl. 438(2), 782-795 (2013)

33. Lee, T.-D., Yang, C.-N.: Statistical theory of equations of state and phase transitions. II. Lattice gas and Ising model. Phys. Rev. 87(3), 410-419 (1952)

34. Lieb, E.H.: Residual entropy of square ice. Phys. Rev. 162(1), 162-172 (1967)

35. Lieb, E.H., Sokal, A.D.: A general lee-Yang theorem for one-component and multicomponent ferromagnets. Comm. Math. Phys. 80(2), 153-179 (1981)

36. Lin, J., Wang, H.: The complexity of Boolean Holant problems with nonnegative weights. SIAM J. Comput. 47(3), 798-828 (2018). Preliminary version appeared in ICALP 2017

37. Margulies, S., Morton, J.: Polynomial-time solvable \#CSP problems via algebraic models and Pfaffian circuits. J. Symbolic Comput. 74, 152-180 (2016)

38. Morton, J.: Pfaffian circuits. CoRR, arXiv:abs/1101.0129 (2011)

39. Onsager, L.: Crystal statistics. I. A two-dimensional model with an order-disorder transition. Phys. Rev. 65(3-4), 117-149 (1944)

40. Schrijver, A.: Characterizing partition functions of the spin model by rank growth. Indag. Math. (N.S.) 24(4), 1018-1023 (2013)

41. Temperley, H.N.V., Fisher, M.E.: Dimer problem in statistical mechanics—an exact result. Philos. Mag. 6, 1061-1063 (1961)

42. Valiant, L.G.: Expressiveness of matchgates. Theor. Comput. Sci. 289(1), 457-471 (2002)

43. Valiant, L.G.: Quantum circuits that can be simulated classically in polynomial time. SIAM J. Comput. 31(4), 1229-1254 (2002)

44. Valiant, L.G.: Accidental Algorthims. In: FOCS, pp. 509-517. IEEE Computer Society (2006)

45. Valiant, L.G.: Holographic algorithms. SIAM J. Comput. 37(5), 1565-1594 (2008)

46. Valiant, L.G.: Some observations on holographic algorithms. Comput. Complex. 27(3), 351-374 (2018). Preliminary version appeared in LATIN 2010

47. Vertigan, D.: The computational complexity of Tutte invariants for planar graphs. SIAM J. Comput. 35(3), 690-712 (2005)

48. Vertigan, D.L.: On the computational complexity of Tutte, Jones, Homfly and Kauffman invariants. $\mathrm{PhD}$ thesis University of Oxford (1991)

49. Welsh, D.: Complexity: Knots, Colourings and Countings. London Mathematical Society Lecture Note Series. Cambridge University Press, Cambridge (1993)

50. Yang, C.-N.: The spontaneous magnetization of a two-dimensional Ising model. Phys. Rev. 85(5), 808-816 (1952)

51. Yang, C.-N., Lee, T.-D.: Statistical theory of equations of state and phase transitions. I. Theory of condensation. Phys. Rev. 87(3), 404-409 (1952)

Publisher's Note Springer Nature remains neutral with regard to jurisdictional claims in published maps and institutional affiliations. 


\section{Affiliations}

Jin-Yi Cai ${ }^{1} \cdot$ Zhiguo Fu $^{2} \cdot$ Heng Guo $^{3}$ (D) $\cdot$ Tyson Williams $^{4}$

Jin-Yi Cai

jyc@cs.wisc.edu

Zhiguo Fu

fuzg432@nenu.edu.cn

Tyson Williams

tdw@cs.wisc.edu

1 Department of Computer Science, University of Wisconsin-Madison, Madison, WI, USA

2 School of Computer Science \& Information Technology, Northeast Normal University, Changchun, China

3 School of Informatics, University of Edinburgh, Edinburgh, UK

4 Blocher Consulting, Champaign, IL, USA 